\title{
Kernel algebras and generalized Fourier-Mukai transforms
}

\author{
Alexander Polishchuk*
}

\begin{abstract}
We introduce and study kernel algebras, i.e., algebras in the category of sheaves on a square of a scheme, where the latter category is equipped with a monoidal structure via a natural convolution operation. We show that many interesting categories, such as $D$ modules, equivariant sheaves and their twisted versions, arise as categories of modules over kernel algebras. We develop the techniques of constructing derived equivalences between these module categories. As one application we generalize the results of [44] concerning modules over algebras of twisted differential operators on abelian varieties. As another application we recover and generalize the results of Laumon [33] concerning an analog of the Fourier transform for derived categories of quasi-coherent sheaves on a dual pair of generalized 1-motives.
\end{abstract}

Mathematics Subject Classification (2010). 14A22 (14F05, 14K05, 18E30).

Keywords. Fourier-Mukai transform, derived category, commutative group scheme, formal groupoid, abelian variety, 1-motive, $D$-modules, equivariant sheaves.

\section{Contents}

1 Preliminaries . . . . . . . . . . . . . . . . . . . . . . 157

1.1 Quasicoherent sheaves on schemes . . . . . . . . . . . . . . 157

1.2 Sheaves on formal schemes . . . . . . . . . . . . . . . . . 159

1.3 Biextensions . . . . . . . . . . . . . . . . . . . . . . . . . . . . . . . . 172

2 Kernel algebras . . . . . . . . . . . . . . . . . . . . . 175

2.1 Convolution for quasi-coherent sheaves . . . . . . . . . . . . . 175

2.2 Kernel algebras . . . . . . . . . . . . . . . . . . . . . . . . . . . . . . . . . . . . . . . . . . . . . . . . . . .

2.3 Čech resolutions . . . . . . . . . . . . . . . . . . . 183

2.4 Convolution for modules over kernel algebras . . . . . . . . . . . . . . . 187

2.5 Extending an equivalence of derived categories to modules over kernel algebras . . . . . . . . . . . . . . . . . . . . . . 194

3 Kernel representations and derived equivalences . . . . . . . . . . . . . . . . . . . 197

3.1 Convolution of sheaves parametrized by formal schemes . . . . . . . . . . . 199

3.2 Kernel representations and convolution algebras . . . . . . . . . . . . . 200

3.3 Equivariant sheaves as modules over kernel algebras . . . . . . . . . . . . . . . . 208

3.4 Twisting geometric actions by 1 -cocycles . . . . . . . . . . . . 211

3.51 -cocycles and Cartier duality . . . . . . . . . . . . . . . . . . . 219

\footnotetext{
* Supported in part by NSF grant.
} 
3.6 Compatibility with open coverings . . . . . . . . . . . . . . 224

3.7 Projective kernel representations and the Fourier-Mukai transform . . . . . . 226

4 Fourier-Mukai duality for orbi-abelian schemes and generalized 1-motives . . . . . 234

4.1 Duality functor on the derived category of commutative proper group schemes over a field . . . . . . . . . . . . . . . . . . . . . . . . . . . . 235

4.2 Orbi-abelian varieties . . . . . . . . . . . . . . . . . 237

4.3 Abstract duality setup . . . . . . . . . . . . . . . . . . . 239

4.4 Fourier-Mukai transform . . . . . . . . . . . . . . . . . . 241

4.5 Fourier-Mukai duality for twisted sheaves . . . . . . . . . . . . . . . . 243

References . . . . . . . . . . . . . . . . . . . . . . . . 248

\section{Introduction}

The classical Morita theory gives a way to construct all equivalences between the categories of modules over rings $A$ and $B$ in terms of $A$ - $B$-bimodules. This theory was generalized to bounded derived categories of module categories by Ricard [45]. On the other hand, starting from the pioneering work of Mukai [38] algebraic geometers got interested in the study of the functors between bounded derived categories of coherent sheaves on projective varieties $X$ and $Y$ associated with complexes of coherent sheaves (kernels) on $X \times Y$ (we refer to such functors as integral transforms). The result of Orlov [40] states that in the case when $X$ and $Y$ are smooth projective varieties all exact equivalences between these derived categories are of this form. Note that the latter theory belongs to the commutative world, although there exist generalizations to stacks (see [32], [12]) and to twisted sheaves (see [17]). There are interesting "noncommutative" categories of geometric origin that are left out from this picture, such as categories of $D$-modules. It seems that there is no straightforward generalization of the above theory to this case. One way to include both the derived Morita theory and the Fourier-Mukai transforms into one framework is by working with dg-categories, as outlined in [49]. In the present paper we propose a more specialized extension of the techniques of integral transforms to a (partially) noncommutative world that does not require passing to dg-categories. Our immediate goal was to understand and generalize two concrete examples, namely, the Fourier duality for generalized 1-motives (see [33]) and for modules over algebras of twisted differential operators on abelian varieties (see [44]). The resulting framework, although much more limited in applicability than the dg-techniques, covers both these examples and their generalizations.

The main idea is quite simple. Composition of integral transforms between derived categories of sheaves corresponds to taking convolution of kernels (see Section 2.1). In particular, we get a monoidal structure on the category of kernels on $X \times X$ with the unit object $\Delta_{*} \mathcal{O}_{X}$, where $\Delta: X \rightarrow X \times X$ is the diagonal. Thus, in the context of derived equivalences it is natural to work with algebra objects with respect to this monoidal structure. This is what we call kernel algebras. The key observation is that 
many interesting categories, such as categories of twisted sheaves on global quotient stacks, can be viewed as suitably defined categories of modules over kernel algebras. Note that a very similar setup involving algebra objects in the monoidal category of sheaf bimodules is used in [50] to define noncommutative $\mathbb{P}^{1}$-bundles.

We observe that a derived equivalence between coherent sheaves on $X$ and $Y$ leads to a correspondence between kernel algebras over $X$ and $Y$. The natural idea then is to try to extend derived equivalences between $X$ and $Y$ to those for modules over kernel algebras over $X$ and $Y$. However, one finds that even defining categories of modules over kernel algebras is not quite straightforward. One possibility would be to work with dg-categories and to try to define dg-categories of modules over suitable dg-models of kernel algebras. In the present paper we follow a more classical approach by considering only those kernel algebras for which it is possible to define first an abelian category of modules. The restriction we impose for this is a kind of flatness condition: the transform from the derived category of sheaves on $X$ to itself defined by our kernel algebra should preserve the abelian subcategory of sheaves. We call kernel algebras with this property pure. Generalizing Theorem 6.5 of [44], we prove that an equivalence of derived categories of sheaves on $X$ and $Y$ extends to a correspondence between kernel algebras over $X$ and $Y$ such that the corresponding derived categories of modules are equivalent, provided both kernel algebras are pure (and other technical assumptions are satisfied). Note that any $D$-algebra in the sense of [7] can be viewed as a pure kernel algebra, so the context of [44] is embedded into our framework. We also show that one can associate a kernel algebra with an action of a finite group scheme $G$ (or a formal group) on the derived category $D_{\mathrm{qc}}(X)$ of quasi-coherent sheaves on $X$ (provided this action is given by integral transforms). In the case when such an action is geometric (i.e., induced by the action of $G$ on $X$ ) the category of modules over the corresponding pure kernel algebra is equivalent to the category of $G$-equivariant sheaves on $X$. More generally, one can associate a pure kernel algebra with an appropriate class of groupoids (resp. formal groupoids). We also consider in detail the situation when $G$ acts on the category of sheaves on $X$ by autoequivalences induced by automorphisms of $X$ combined with tensoring by line bundles. Modules over the corresponding $G$-algebras can be interpreted as twisted $G$-equivariant sheaves on $X$.

Let us explain how kernel algebras can be used to establish an analog of the Fourier-Mukai duality for the derived categories of sheaves on generalized 1-motives considered in [33]. We consider a slightly broader class of generalized 1-motives than in [33], namely, complexes $[G \rightarrow E]$ concentrated in degrees -1 and 0 , where $G$ is a commutative formal group (satisfying some finiteness assumptions) and $E$ is a commutative algebraic group. ${ }^{1}$ Quasicoherent sheaves on $[G \rightarrow E]$ are simply $G$ equivariant quasi-coherent sheaves on $E$, so they can be described by an appropriate pure kernel algebra on $E$. Note that $E$ is an extension of an abelian variety $A$ by an affine algebraic group. Using an appropriate notion of the push-forward for kernel

\footnotetext{
${ }^{1}$ Thus, unlike in [33], we allow $G$ to have torsion and $E$ to be nonconnected.
} 
algebras we can equivalently describe sheaves on $K=[G \rightarrow E]$ as modules over some pure kernel algebra $\mathcal{A}(K)$ on $A$. The dual 1-motive $\mathbb{D}(K)$ is presented by a complex $\left[G^{\prime} \rightarrow E^{\prime}\right]$, where $E^{\prime}$ is an extension of the dual abelian variety $\hat{A}$ by an affine group. Applying the same procedure to $\mathbb{D}(K)$ we get a kernel algebra $\mathcal{A}(\mathbb{D}(K))$ on $\hat{A}$ responsible for sheaves on $\mathbb{D}(K)$. Furthermore, we interpret $\mathcal{A}(K)$ (resp. $\mathcal{A}(\mathbb{D}(K))$ ) as the kernel algebra associated with a homomorphism from $G \times G^{\prime}$ to Auteq $\left(D_{\mathrm{qc}}(A)\right)\left(\right.$ resp. Auteq $\left.\left(D_{\mathrm{qc}}(\hat{A})\right)\right)$. Thus, sheaves on $K($ resp. $\mathbb{D}(K))$ can be viewed as twisted $G \times G^{\prime}$-equivariant sheaves on $A$ (resp. $\hat{A}$ ). Using this point of view we check that $\mathcal{A}(K)$ and $\mathcal{A}(\mathbb{D}(K))$ correspond to each other under the standard Fourier-Mukai transform which gives an equivalence between the derived categories of sheaves on $A$ and on $\hat{A}$. This immediately leads to the desired equivalence of derived categories of sheaves on $K$ and on $\mathbb{D}(K)$.

The paper is organized as follows. In Section 1 we collect some results on quasicoherent sheaves over schemes and formal schemes, as well as few facts about biextensions. Most of these results are well known. However, in some cases we had to prove simple extensions of the known theorems to new situations (for example, we give three versions of the base change formula for sheaves on formal schemes). Also, we prove here some auxiliary statements involving duality for sheaves on formal schemes. Section 2 develops the theory of kernel algebras. Here we define a convolution operation for modules over pure kernel algebras satisfying an additional technical assumption. The main result of this section is Theorem 2.5.1, which extends equivalences between derived categories of sheaves on schemes $X$ and $Y$ to derived categories of modules over kernel algebras over $X$ and $Y$. In Section 3 we define the notion of a kernel representation of a group scheme (or a formal group) $G$ over a scheme $X$, which is a scheme-theoretic version of a homomorphism from a discrete group to Auteq $\left(D_{\mathrm{qc}}(X)\right)$. In the case when $G$ is a finite flat group scheme (resp. formal group) we construct a kernel algebra over $X$ associated with a kernel representation. We study in particular such kernel algebras corresponding to homomorphisms from $G$ to $\operatorname{Aut}(X) \ltimes \operatorname{Pic}(X)$, or equivalently, with 1-cocycles of $G$ with values in $\operatorname{Pic}(X)$. In the case when $G$ is commutative the interplay between such algebras and the Cartier duality plays a crucial rule in applications to 1-motives. On the other hand, Theorem 3.3.1 establishes an equivalence between the category of sheaves equivariant with respect to an action of a groupoid (resp. formal groupoid) on $X$ and modules over the corresponding kernel algebra. We also construct an equivalence of derived categories for twisted $G$-equivariant sheaves on a pair of dual abelian schemes (see Theorem 3.7.3) generalizing equivalences for modules over twisted differential operators considered in [44]. Finally, in Section 4 we apply the developed techniques to constructing an analog of Fourier-Mukai transform for generalized 1-motives and to generalizing Theorem 3.7.3 to this context.

Acknowledgment. I am grateful to Alexander Beilinson, Oren Ben-Bassat, Joseph Lipman and Daniel Hernandez Ruiperez for useful discussions. I also thank the referee for helpful suggestions. 
Notations and conventions. All schemes in this paper are assumed to be noetherian (this applies also to the fibered products appearing in our constructions), and all formal schemes are assumed to be locally noetherian. For our conventions on formal groups see the beginning of Section 3. By an algebraic group over a field $k$ we mean a group scheme of finite type over $k$. By flat topology we mean the fppf topology.

Starting from Section 2, all functors are assumed to be derived. Thus, for a morphism $f$ between schemes $f_{*}$ (resp. $f^{*}$ ) will denote the derived push-forward (resp. pull-back) of complexes of sheaves of $\mathcal{O}$-modules. Similarly, $\otimes$ will denote the derived tensor product (also starting from Section 2). Recall that these functors are defined on the unbounded derived categories of $\mathcal{O}$-modules (see [48] and Chap. 2 of [35]). Similar conventions are applied for formal schemes.

All inductive (resp. projective) systems are assumed to be small and filtered. We denote by lim (resp. lim) the inductive (resp. projective) limit of an inductive (resp. projective) system.

\section{Preliminaries}

1.1. Quasicoherent sheaves on schemes. Our primary source regarding derived categories of quasi-coherent sheaves on schemes are the notes [35] (although we are not aiming at the same level of generality). Recall that we assume all schemes to be noetherian, so every morphism between them is quasi-compact and quasi-separated (see [25], (6.1.13)). We denote by $\mathrm{Q} \operatorname{coh}(X)(\operatorname{resp} . \operatorname{Coh}(X))$ the category of quasicoherent (resp. coherent) sheaves of $\mathcal{O}$-modules on a scheme $X$. We denote by $D_{\mathrm{qc}}(X)$ (resp. $\left.D_{c}(X)\right)$ the full subcategory in the derived category of $\mathcal{O}$-modules consisting of complexes with quasi-coherent (resp. coherent) cohomology.

First, we observe that the standard (derived) functors with complexes of $\mathcal{O}$ modules preserve the subcategories $D_{\mathrm{qc}}$. For the pull-back functors and for the tensor product this requires no assumptions on the schemes involved (see [35], 3.9.1 and 2.5.8). For the push-forward functor this is true because we consider only noetherian schemes ([35], 3.9.2). For the same reason for a map $f: X \rightarrow Y$ the functor $R f_{*}: D_{\mathrm{qc}}(X) \rightarrow D_{\mathrm{qc}}(Y)$ is bounded above ([35], 3.9.2), i.e., it sends $D_{\mathrm{qc}}^{\leq 0}(X)$ to $D_{\mathrm{qc}}^{\leq n}(Y)$ for some $n$ (it is also bounded below for trivial reasons). In the case when $f: X \rightarrow Y$ is an affine morphism the functor $f_{*}$ is exact and the induced functor $D_{\mathrm{qc}}(X) \rightarrow D_{\mathrm{qc}}(Y)$ is conservative (this follows from the fact that for an affine scheme $X=\operatorname{Spec}(A)$ the functor of global sections induces an equivalence of $D_{\mathrm{qc}}(X)$ with $D(A$-mod $)$ ).

The following result follows immediately from Lemma 3.9.3.1 in [35]. Note that the crucial case of $R^{0} f_{*}$ traces back to the proof of Theorem 3.10 in [24].

Lemma 1.1.1. Let $f: X \rightarrow Y$ be a morphism of schemes. Then $R^{i} f_{*}$ commutes with inductive limits. 
We will use the following versions of the projection formula and the base change formula.

Theorem 1.1.2 ([35], 3.9.4). Let $f: X \rightarrow Y$ be a morphism of schemes. Then the natural map

$$
R f_{*} F \otimes^{\mathbb{L}} G \rightarrow R f_{*}\left(F \otimes^{\mathbb{L}} L f^{*} G\right)
$$

is an isomorphism for $F \in D_{\mathrm{qc}}(X), G \in D_{\mathrm{qc}}(Y)$.

Theorem 1.1.3 ([35], 3.9.5 and 3.10.3; [5], Prop. A.85). Assume that we have a cartesian diagram

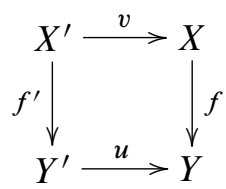

where either $u$ or $f$ is flat. Then for $F \in D_{\mathrm{qc}}(X)$ the base change map $L u^{*} R f_{*} F \rightarrow$ $R f_{*}^{\prime} L v^{*} F$ is an isomorphism.

A scheme $X$ is called semi-separated if the diagonal morphism $X \rightarrow X \times X$ is affine. It is easy to see that any morphism from an affine scheme to a semi-separated scheme is affine. For a quasi-coherent sheaf on a semi-separated scheme one can construct a quasi-isomorphic complex of flat quasi-coherent sheaves (using Čech resolution, see [1], Prop. 1.1). This easily implies the following statement (see also [37], Prop. 16).

Lemma 1.1.4. Let $X$ be a semi-separated scheme. Then for every quasi-coherent sheaf $F$ on $X$ there exists a surjection $P \rightarrow F$, where $P$ is flat quasi-coherent. Moreover, this surjection can be constructed functorially in $F$.

Recall that for a finite morphism $f: X \rightarrow Y$ the functor $f_{*}: D_{\mathrm{qc}}(X) \rightarrow D_{\mathrm{qc}}(Y)$ admits the right adjoint $f^{!}: D_{\mathrm{qc}}(Y) \rightarrow D_{\mathrm{qc}}(X)$. We need the following simple fact about this functor.

Proposition 1.1.5. Let $f: X \rightarrow Y$ be a finite flat morphism. Then $f^{!} \mathcal{O}_{Y}$ is a coherent sheaf on $X$, flat over $Y$, and for $F \in D_{\mathrm{qc}}(Y)$ the natural morphism

$$
f^{!} \mathcal{O}_{Y} \otimes f^{*} F \rightarrow f^{!} F
$$

in $D_{\mathrm{qc}}(X)$ is an isomorphism. Given any morphism $u: U \rightarrow Y$ let us consider the cartesian square

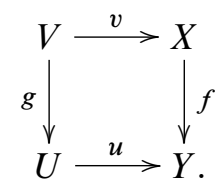

Then one has a natural isomorphism of functors $L v^{*} f^{!} \simeq g^{!} L u^{*}$. 
Proof. It is enough to check that our map becomes an isomorphism after applying $f_{*}$. By the local duality isomorphism we have

$$
f_{*} f^{!} F \simeq \mathscr{H o m}\left(f_{*} \mathcal{O}_{X}, F\right) .
$$

Note that $f_{*} \mathcal{O}_{X}$ is a vector bundle, so the natural map

$$
\operatorname{Hom}\left(f_{*} \mathcal{O}_{X}, \mathcal{O}_{Y}\right) \otimes F \rightarrow \operatorname{Hom}\left(f_{*} \mathcal{O}_{X}, F\right)
$$

is an isomorphism. It remains to observe that the target of this map is isomorphic to $f_{*}\left(f^{!} \mathcal{O}_{X} \otimes f^{*} F\right)$ (by the projection formula).

Given the base change diagram as above, we have a natural morphism $v^{*} f^{!} \mathcal{O}_{Y} \rightarrow$ $g^{!} \mathcal{O}_{U}$ corresponding by adjunction to

$$
g_{*} v^{*} f^{!} \mathcal{O}_{Y} \simeq u^{*} f_{*} f^{!} \mathcal{O}_{Y} \rightarrow \mathcal{O}_{U}
$$

To check that it is an isomorphism it is enough to compare the push-forwards by $g$. It remains to use the isomorphism

$$
\operatorname{Hom}\left(g_{*} \mathcal{O}_{V}, \mathcal{O}_{U}\right) \simeq u^{*} \operatorname{Hom}\left(f_{*} \mathcal{O}_{X}, \mathcal{O}_{Y}\right)
$$

that follows from $g_{*} \mathcal{O}_{V} \simeq u^{*} f_{*} \mathcal{O}_{X}$.

1.2. Sheaves on formal schemes. We use the definitions of [25] concerning formal schemes. All formal schemes considered in the present paper are assumed to be locally noetherian. Thus, our formal schemes are locally of the form $\operatorname{Spf}(A)$, where $A$ is an adic noetherian ring. Following [2] we say that a morphism $f: X \rightarrow Y$ between formal schemes is of pseudofinite type (resp. pseudofinite) if there exist ideals of definition $\mathscr{g}_{X} \subset \mathcal{O}_{X}, \mathscr{g}_{Y} \subset \mathcal{O}_{Y}$ such that $\mathscr{g}_{Y} \mathcal{O}_{X} \subset \mathscr{g}_{X}$ and the induced map of schemes $\left(X, \mathcal{O}_{X} / \mathscr{G}_{X}\right) \rightarrow\left(Y, \mathcal{O}_{Y} / \mathscr{G}_{Y}\right)$ is of finite type (resp. finite).

By a sheaf on $X$ we always mean a sheaf of $\mathcal{O}_{X}$-modules. We denote by $A(X)$ the abelian category of sheaves on $X$ and by $D(X)$ its (unbounded) derived category.

Following [2] we work with several full subcategories of $A(X)$ and $D(X)$. First, we consider the categories $A_{\mathrm{qc}}(X) \subset A(X)$ (resp. $\left.A_{\mathrm{c}}(X)\right)$ of quasi-coherent (resp. coherent) sheaves. Next, let us recall the definition of torsion sheaves on $X$. For a coherent ideal sheaf $\mathcal{g} \subset \mathcal{O}_{X}$ one defines the subfunctor of the identity functor

$$
\Gamma_{g} \mathcal{F}=\lim _{n} \mathscr{H o m}\left(\mathcal{O}_{X} / \mathscr{g}^{n}, \mathcal{F}\right) .
$$

This functor is left exact and depends only on the topology on $\mathcal{O}_{X}$ defined by $\mathcal{F}$. By definition, $\mathcal{F}$-torsion sheaves are sheaves $\mathcal{F}$ satisfying $\Gamma_{\mathscr{g}} \mathcal{F}=\mathcal{F}$. For a formal scheme $X$ torsion sheaves on $X$ are defined as $\mathcal{f}$-torsion sheaves, where $\mathcal{g}$ is an ideal of definition for $X$ (recall that on locally noetherian formal schemes ideals of definition exist globally, see [25], Prop. (10.5.4)). We denote by $A_{\mathrm{qct}}(X) \subset A_{\mathrm{qc}}(X)$ the subcategory of torsion quasi-coherent sheaves. 
Although we are mainly interested in the categories $A_{\mathrm{c}}(X)$ and $A_{\mathrm{qct}}(X)$, it is sometimes convenient to work with the subcategory $A_{\overrightarrow{\mathrm{c}}}(X) \subset A_{\mathrm{qc}}(X)$ of sheaves that can be presented as direct limits of coherent sheaves. Note that $A_{\vec{c}}(X)$ contains both $A_{\mathrm{c}}(X)$ and $A_{\text {qct }}(X)$ (see Lemma 5.1.4 of [2]). Also, locally every quasi-coherent sheaf is in $A_{\overrightarrow{\mathrm{c}}}$ (see Cor. 3.1.4 of [2]).

For a pair of quasi-coherent sheaves $F$ and $G$ on a formal scheme $X$ we set

$$
\widehat{\operatorname{Hom}}(F, G)=\underset{\leftarrow}{\lim _{n}} \operatorname{Hom}\left(F, G \otimes \mathcal{O}_{X} / g^{n}\right),
$$

where $\mathcal{G}$ is an ideal of definition for $X$. It is easy to see that these groups do not depend on a choice of an ideal of definition. If $i_{n}: X_{n} \hookrightarrow X$ is a closed embedding of the subscheme corresponding to the ideal sheaf $g^{n}$ then we have

$$
\widehat{\operatorname{Hom}}(F, G)=\underset{\leftarrow}{\lim _{n}} \operatorname{Hom}\left(i_{n}^{*} F, i_{n}^{*} G\right)
$$

Hence, we have a natural composition law for $\widehat{\operatorname{Hom}}$. Given an element $\alpha \in \widehat{\operatorname{Hom}}(F, G)$ then for every morphism $f: Y \rightarrow X$ of formal schemes we have the induced element $f^{*} \alpha \in \widehat{\operatorname{Hom}}\left(f^{*} F, f^{*} G\right)$ (use ideals of definition $\mathcal{g}_{X} \in \mathcal{O}_{X}$ and $\mathcal{g}_{Y} \in \mathcal{O}_{Y}$ such that $\left.\mathcal{I}_{X} \mathcal{O}_{Y} \subset \mathcal{I}_{Y}\right)$.

For $*=c, q c, \overrightarrow{\mathrm{c}}, q c t$ we denote by $D_{*}(X)$ the full subcategory of complexes with cohomology in $A_{*}(X)$.

Let $R \Gamma_{\mathcal{g}}$ denote the derived functor to $\Gamma_{\mathfrak{g}}$. For a complex of sheaves with $\mathcal{g}$ torsion cohomology the natural map $R \Gamma_{\mathcal{J}} F \rightarrow F$ is an isomorphism (see the proof of Proposition 5.2.1 (a) of [2]). In the case when $g$ is an ideal of definition of $X$ we set $R \Gamma_{X}^{\prime}=R \Gamma_{\mathcal{g}}$. For $F \in D_{\mathrm{qc}}(X)$ we have $R \Gamma_{X}^{\prime}(F) \in D_{\mathrm{qct}}(X)$. Also, for such $F$ the natural map $R \Gamma_{X}^{\prime}(F) \rightarrow F$ is an isomorphism if and only if $F \in D_{\mathrm{qct}}(X)$ (see Prop. 5.2.1 of [2]).

Since we consider only locally noetherian formal schemes, by Cor. 3.1.2 of [1], the natural map

$$
E \otimes^{\mathbb{L}} R \Gamma_{X}^{\prime}(F) \rightarrow R \Gamma_{X}^{\prime}\left(E \otimes^{\mathbb{L}} F\right)
$$

is an isomorphism for all $E, F \in D(X)$.

Lemma 1.2.1. Let $X$ be a formal scheme The subcategories $D_{c}(X), D_{\overrightarrow{\mathrm{c}}}(X)$ and $D_{\mathrm{qc}}$ are all stable under the derived tensor product functor $\otimes^{\mathbb{L}}: D(X) \times D(X) \rightarrow D(X)$. Also, if $A \in D(X)$ has $\mathcal{A}$-torsion cohomology, where $\mathcal{A} \subset \mathcal{O}_{X}$ is a coherent ideal sheaf then the same is true for $A \otimes^{\mathbb{L}} B$ for any $B \in D(X)$.

Proof. Using the formula $A \otimes^{\mathbb{L}} B=\lim _{m, n} \tau_{\leq m} A \otimes^{\mathbb{L}} \tau_{\leq n} B$ and the spectral sequence we can reduce the first assertion to showing that for $F, G \in A_{*}(X)$ (where $*=\mathrm{c}$, $\overrightarrow{\mathrm{c}}$, qc) one has $\operatorname{Tor}_{n}(F, G) \in A_{*}(X)$. In the case $F$ and $G$ are coherent sheaves this is clear by reducing to the affine case, where the tensor product simply corresponds to the tensor product over the corresponding ring (see [25], Prop. (10.10.2.3)). The 
case $F, G \in A_{\overrightarrow{\mathrm{c}}}(X)$ follows because $\mathcal{T} r_{n}$ commutes with direct limits (see 2.5 of [35]). Since locally any quasi-coherent sheaf is in $A_{\overrightarrow{\mathrm{c}}}(X)$, this also implies the case of $*=q c$.

As above, the last statement can be reduced to the case when $A$ is a $\mathcal{J}$-torsion sheaf. Now the assertion follows by choosing a $q$-flat resolution of $B$ and using the similar statement for the underived tensor product of sheaves.

For a morphism $f: X \rightarrow Y$ of formal schemes one has the derived functors of push-forward $R f_{*}: D(X) \rightarrow D(Y)$ and pull-back $L f^{*}: D(Y) \rightarrow D(X)$. In the following proposition we collect some of their properties (mostly proved in [2]).

Proposition 1.2.2. (i) One has $L f^{*} D_{\overrightarrow{\mathrm{c}}}(Y) \subset D_{\mathrm{qc}}(X)$. If in addition, $Y$ is affine then $L f^{*} D_{\overrightarrow{\mathrm{c}}}(Y) \subset D_{\overrightarrow{\mathrm{c}}}(X)$. On the other hand, if $f$ is flat then $f^{*} D_{c}(Y) \subset D_{c}(X)$ and $f^{*} D_{\overrightarrow{\mathrm{c}}}(Y) \subset D_{\overrightarrow{\mathrm{c}}}(X)$.

(ii) Let $\mathcal{g} \subset \mathcal{O}_{Y}$ be a coherent sheaf of ideals, and let $D_{\mathcal{g}}(Y) \subset D(Y)$ (resp. $\left.D_{\mathcal{J O}_{X}}(X) \subset D(X)\right)$ denote the subcategory of complexes with $\mathcal{G}$-torsion (resp. $\mathcal{G}_{\mathcal{O}_{X}-}$ torsion) cohomology. There is an isomorphism of functors

$$
L f^{*} R \circ \Gamma_{\mathcal{g}} \simeq R \Gamma_{\mathcal{j} \mathcal{O}_{X}} \circ L f^{*} .
$$

Hence, $L f^{*} D_{\mathcal{J}}(Y) \subset D_{\mathfrak{J}_{X}}(X)$. If $X$ is noetherian then we also have

$$
R \Gamma_{\mathcal{g}} \circ R f_{*} \simeq R f_{*} R \circ \Gamma_{\mathcal{J} \mathcal{O}_{Y}},
$$

so in this case $R f_{*} D_{\mathcal{J O}_{X}}(X) \subset D_{\mathcal{g}}(Y)$.

(iii) Assume that $X$ and $Y$ are noetherian. Then the functor $R f_{*}$ is bounded on $D_{\overrightarrow{\mathrm{c}}}(X)$.

(iv) Assume that $f$ is quasi-compact. Then $R f_{*} D_{\text {qct }}(X) \subset D_{\text {qct }}(Y)$.

(v) Assume that $f$ is quasi-compact. Then the functor $R f_{*}$ commutes with small direct sums in $D_{\overrightarrow{\mathrm{c}}}(X)$. Also, in this case the functors $R^{i} f_{*}$ commute with (small filtered) direct limits.

(vi) If $f$ is proper then $R f_{*} D_{c}(X) \subset D_{c}(Y)$. If in addition $X$ and $Y$ are noetherian then $R f_{*} D_{\overrightarrow{\mathrm{c}}}(X) \subset D_{\overrightarrow{\mathrm{c}}}(Y)$.

(vii) If $f$ is affine then $f_{*}$ is exact on $D_{\overrightarrow{\mathrm{c}}}(X)$ and $f_{*} D_{\overrightarrow{\mathrm{c}}}(X) \subset D_{\mathrm{qc}}(Y)$.

Proof. (i) The first two assertions follow from Prop. 3.3.5 of [2]. For the last assertion we use the well-known facts that $f^{*} A_{\mathrm{c}}(Y) \subset A_{\mathrm{c}}(X)$ and $f^{*}$ commutes with direct limits.

(ii) See Prop. 5.2.8 (b),(d) of [2].

(iii) See Prop. 3.4.3 (b) of [2].

(iv) Since the assertion is local in $Y$, we can assume that $X$ and $Y$ are noetherian. Then we can use Prop. 5.2.6 of [2].

(v) Since the assertion is local in $Y$, we can assume that $X$ and $Y$ are noetherian. Then the first assertion follows from Prop. 3.5.2 of [2]. The second assertion is checked exactly as in the case of schemes (see Lemma 1.1.1). 
(vi) Since the first assertion is local in $Y$, it is enough to consider the case when $X$ and $Y$ are noetherian, so we can use Prop. 3.5.1 of [2].

(vii) We can assume that both $X$ and $Y$ are affine: $X=\operatorname{Spf}(A)$ and $Y=\operatorname{Spf}(B)$. Exactness of $f_{*}$ follows from Lemma 3.4.2 of [2]. By part (v), it is enough to check that $f_{*} A_{\mathrm{c}}(X) \subset A_{\mathrm{qc}}(Y)$. Every coherent sheaf on $X$ is of the form $M^{\Delta}$ for some finitely generated $A$-module $M$. It is easy to see that $f_{*}\left(M^{\Delta}\right)$ is the $\mathcal{O}_{Y}$-module associated with $M$ viewed as a $B$-module via the construction of Prop. 3.2 of [52]. Hence, $f_{*} M \in A_{\overrightarrow{\mathrm{c}}}(Y) \subset A_{\mathrm{qc}}(Y)$.

We will also use push-forwards with quasi-compact support in a very special case. Instead of developing the general theory we will give an ad hoc definition for this case.

Definition. (i) Let $\mathbf{P}$ be some property of morphisms between formal schemes, local over base, and let $f: X \rightarrow Y$ be a morphism of formal schemes. We say that $f$ is ldu-P ('ldu' means locally disjoint union) if there exists an open covering $\left(U_{i}\right)$ of $Y$ such that for every $i, f^{-1}\left(U_{i}\right)$ is a disjoint union of formal schemes $V_{i, j}$ such that all the maps $\left.f\right|_{V_{i, j}}: V_{i, j} \rightarrow U_{i}$ have property $\mathbf{P}$. Note that if the property $\mathbf{P}$ is stable under base changes then the same is true for the property "ldu-P".

(ii) Let $f: X \rightarrow Y$ be an ldu-quasi-compact morphism between formal schemes. Let us consider the subfunctor

$$
f_{\sigma *}: A(X) \rightarrow A(Y)
$$

in $f_{*}$, where $f_{\sigma *}(F) \subset f_{*}(F)$ is the subsheaf of sections with quasi-compact support over $Y$. This functor is left exact and we denote by $R f_{\sigma *}: D(X) \rightarrow D(Y)$ its derived functor. Note that $Y$ can be covered by open subsets $U \subset Y$ such that $f^{-1}(U)=\sqcup V_{j}$ where each $f_{j}=\left.f\right|_{V_{j}}: V_{j} \rightarrow U$ is quasi-compact, and we have

$$
\left.R f_{\sigma *}(F)\right|_{U} \simeq \bigoplus_{j} R f_{j *}\left(\left.F\right|_{V_{j}}\right) .
$$

Observe also that the definition of $f_{\sigma *}$ is local in $Y$. Hence, Proposition 1.2.2 (iv) implies that $R f_{\sigma *} D_{\text {qct }}(X) \subset D_{\text {qct }}(Y)$. If $f$ is quasi-compact then $f_{\sigma *}=f_{*}$. In the case when $f$ is ldu-pseudofinite we will denote $f_{\sigma *}$ by $f_{\text {! }}$ (note that this functor is exact).

One can immediately generalize many properties of the push-forward functors for formal schemes to the functors $R f_{\sigma *}$ for ldu-quasi-compact morphisms. For example, for ldu-quasi-compact maps of formal schemes $f: X \rightarrow Y$ and $g: Z \rightarrow X$ one has $R(f \circ g)_{\sigma *} \simeq R f_{\sigma *} \circ R g_{\sigma *}$.

We now turn to versions of the projection and base change formulae for formal schemes. 
Theorem 1.2.3. Let $f: X \rightarrow Y$ be a quasi-compact map of formal schemes. Then for $F \in D_{\overrightarrow{\mathrm{c}}}(X)$ and $G \in D_{\overrightarrow{\mathrm{c}}}(Y)$ the natural map

$$
G \otimes^{\mathbb{L}} R f_{*} F \rightarrow R f_{*}\left(L f^{*} G \otimes^{\mathbb{L}} F\right)
$$

is an isomorphism. The similar result holds if $f$ is ldu-quasi-compact and $R f_{*}$ is replaced with $R f_{\sigma *}$.

Proof. The question is local in $Y$, so we can assume that $Y$ is affine and $X$ is noetherian (replacing $X$ by one of its connected component in the second case). Note that $L f^{*} G \in D_{\overrightarrow{\mathrm{c}}}(X)$ by Proposition 1.2.2 (i). Hence, $L f^{*} G \otimes^{\mathbb{Z}} F \in D_{\overrightarrow{\mathrm{c}}}(X)$ by Lemma 1.2.1. First, assume that both $F$ and $G$ are bounded above. Fix $F$. Then both sides respect coproducts as functors in $G$ (use [2], Prop. 3.5.2). If $G=\mathcal{O}_{Y}$ then the statement is clear. Now use the fact that every object of $A_{\overrightarrow{\mathrm{c}}}(Y)$ is a quotient of a free module plus boundedness above of both sides (by the way out argument - see [35], 1.11.3.1). The case when $F$ and $G$ are unbounded can be deduced from this as in [35], 3.9.4, with $D_{\mathrm{qc}}$ replaced by $D_{\overrightarrow{\mathrm{c}}}$.

Remark. In the above theorem it is enough to assume that there exists an open covering $\left(U_{i}\right)$ of $Y$ such that $\left.G\right|_{U_{i}} \in D_{\overrightarrow{\mathrm{c}}}\left(U_{i}\right)$ and $F \in D_{\overrightarrow{\mathrm{c}}}\left(f^{-1}\left(U_{i}\right)\right)$ (since the question is local in $Y$ ). For example, if $f$ is a closed embedding then the projection formula holds for $F \in D_{\mathrm{qc}}^{b}(X)$ and $G \in D_{\mathrm{qc}}^{b}(Y)$.

We will use two versions of the flat base change formula for sheaves on formal schemes based on Proposition 7.2 of [2]. In addition we prove a base change formula for flat adic morphisms (part (i) (b) of the theorem below).

Theorem 1.2.4. (i) Let

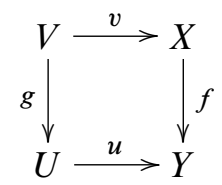

be a cartesian diagram of formal schemes.

(a) Assume that $f$ is proper and $u$ is flat. Then for every $F \in D_{\overrightarrow{\mathrm{c}}}(X)$ the natural map

$$
\theta_{F}: L u^{*} R f_{*} F \rightarrow R g_{*} L v^{*} F
$$

is an isomorphism.

(b) Now assume that $f$ is flat. In addition assume that $f$ is adic and quasicompact (resp. proper). Then the map $\theta_{F}$ is an isomorphism for every $F \in D_{\mathrm{qct}}(X)$ (resp. $\left.F \in D_{\overrightarrow{\mathrm{c}}}(X)\right)$.

(ii) Consider a cartesian diagram as above, where $f$ is of pseudofinite type and $u$ is flat. Assume also that $\mathcal{I}$ is a coherent sheaf of ideals on $U$ such that if $\mathcal{I}$ is an ideal of definition on $Y$ then $\mathcal{B} \mathcal{O}_{U}+\mathcal{I}$ is an ideal of definition on $U$. Then for 
every $F \in D_{\mathrm{qct}}(X)$ and every $G \in D_{\mathrm{qc}}(U)$ such that $G$ has $\mathcal{I}$-torsion cohomology sheaves, the map induced by the base change map

$$
u^{*} R f_{*} F \otimes^{\mathbb{L}} G \rightarrow R g_{*} v^{*} F \otimes^{\mathbb{L}} G
$$

is an isomorphism.

(iii) The assertions of (i) and (ii) also hold if $R f_{*}$ (resp. $R g_{*}$ ) is replaced with $R f_{\sigma *}\left(\right.$ resp. $\left.R g_{\sigma *}\right)$, and every property $\boldsymbol{P}$ of $f$ is replaced with "ldu-P".

Proof. Let us observe that all the assertions are local in $Y$ and $U$, so in the proof we can (and will) assume them to be noetherian.

(i) (a) Using Proposition 7.2 (c) of [2] we see that $R \Gamma_{U}^{\prime} \theta_{F}$ is an isomorphism. By Prop. 6.2.1 of [2] this implies that $\theta_{F}$ itself is an isomorphism when $F$ is a coherent sheaf. Indeed, in this case the source and the target of $\theta_{F}$ are in $D_{c}\left(Y^{\prime}\right)$ by Proposition 1.2.2(vi). It follows that $\theta_{F}$ is an isomorphism for $F \in A_{\overrightarrow{\mathrm{c}}}(X)$ (using Proposition 1.2.2(v)). By Proposition 1.2.2 (iii), the source and the target of $\theta_{F}$ are bounded functors, so we can finish the proof by the way out argument (see [35], 1.11.3).

(b) Since the assertion is local in $U$, we can assume it to be affine. Then it is enough to check that the map $u_{*} \theta_{F}$ on $Y$ is an isomorphism. Note that $R f_{*} F \in D_{\mathrm{qct}}(Y)$ by Proposition 1.2.2 (iv) (resp. $R f_{*} F \in D_{\overrightarrow{\mathrm{c}}}(Y)$ by Proposition 1.2.2(vi)). Therefore, using the projection formula (see Theorem 1.2.3) we can identify the source of $u_{*} \theta_{F}$ with $u_{*} \mathcal{O}_{U} \otimes^{\mathbb{L}} R f_{*} F$ and the target of $u_{*} \theta_{F}$ with $R f_{*}\left(v_{*} \mathcal{O}_{V} \otimes^{\mathbb{L}} F\right)$. It remains to use the isomorphism $v_{*} \mathcal{O}_{V} \simeq f^{*} u_{*} \mathcal{O}_{U}$ (recall that $f$ is adic) and the projection formula again.

(ii) By Proposition 7.2 (b) of [2], the map

$$
R \Gamma_{U}^{\prime} u^{*} R f_{*} F \rightarrow R \Gamma_{U}^{\prime} R g_{*} v^{*} F
$$

is an isomorphism. Using the isomorphism (1.2.2) we see that the map (1.2.3) becomes an isomorphism after applying $R \Gamma_{U}^{\prime}$. It remains to check that the target and the source of (1.2.3) have torsion cohomology sheaves. Since $G$ has $\mathcal{I}$-torsion cohomology sheaves, it is enough to check that the cohomology of $u^{*} R f_{*} F$ and $R g_{*} v^{*} F$ are $\mathcal{g} \mathcal{O}_{U}$-torsion sheaves. For $u^{*} R f_{*} F$ this follows from the fact that $R f_{*} F$ has J-torsion cohomology (see Proposition 1.2.2 (ii)). On the other hand, by the same proposition, to check that the cohomology of $R g_{*} v^{*} F$ are $\mathcal{A} \mathcal{O}_{U}$-torsion sheaves it is enough to check that $v^{*} F$ has $\mathcal{g} \mathcal{O}_{V}$-torsion cohomology. But this immediately follows from the assumption that $F$ has torsion cohomology on $X$.

(iii) Shrinking $Y$ we can assume that all the connected components $X_{i}$ of $X$ are noetherian. Then replacing $X$ by $X_{i}$ and $V$ by $V_{i}=U \times_{Y} X_{i}$, we reduce ourselves to the situation considered in (i) and (ii).

Remark. One situation where the case (ii) of the above theorem applies is this. Assume that $X, Y$ and $Z$ are formal schemes over a usual scheme $S$, where $Z$ is 
flat over $S$. Then for a map $f: X \rightarrow Y$ of pseudofinite type we can consider the cartesian diagram

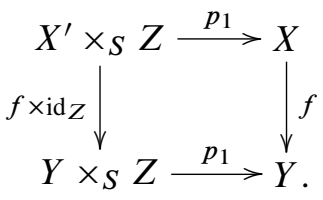

Then for every $F \in D_{\mathrm{qct}}(X)$ and $G \in D_{\mathrm{qct}}(Z)$ the natural map

$$
p_{1}^{*} R f_{*} F \otimes \mathbb{L} L p_{2}^{*} G \rightarrow R\left(f \times \mathrm{id}_{Z}\right)_{*} p_{1}^{*} F \otimes^{\mathbb{L}} L p_{2}^{*} G
$$

is an isomorphism. Similar assertion holds for $R f_{\sigma *}$ instead of $R f_{*}$ provided $f$ is of ldu-pseudofinite type.

Finally, we need a version of Proposition 1.1.5 for a certain class of morphisms between formal schemes. For the kind of morphisms we are interested in the situation is much simpler than in the general duality setup considered in [2] (in particular, in our case the functor $f^{!}$can be easily constructed at the level of abelian categories of sheaves).

Definition. Let $f: X \rightarrow Y$ be a flat morphism of formal schemes. We say that $f$ is nicely ind-finite if each connected component of $X$ is affine over $Y$ and can be presented as the inductive limit of a system of closed formal subschemes $X_{0} \subset X_{1} \subset$ $\cdots \subset X$, where each $X_{n}$ is flat and finite over $Y$. We say that $f$ is locally nicely ind-finite if the above condition holds locally in $Y$. We say that a formal scheme $X$ over a usual scheme $S$ is (locally) nicely ind-finite flat over $S$ if the morphism $\pi: X \rightarrow S$ is such.

It is easy to see that the property of a flat morphism to be nicely ind-finite (resp. locally nicely ind-finite) is preserved under arbitrary base changes. Also, if $f$ is locally nicely ind-finite morphism then it is ldu-pseudofinite, so we have the exact functor $f !$.

Proposition 1.2.5. (i) Let $f: X \rightarrow Y$ be a finite flat morphism of formal schemes. There exists a natural coherent sheaf $f^{!} \mathcal{O}_{Y}$ on $X$, equipped with a morphism

$$
f_{*} f^{!} \mathcal{O}_{Y} \rightarrow \mathcal{O}_{Y},
$$

such that for every map from a usual scheme $Y^{\prime}$ to $Y$ the pull-back of $f^{!} \mathcal{O}_{Y}$ to $X^{\prime}=Y^{\prime} \times_{Y} X$ is isomorphic to $\left(f^{\prime}\right)^{!} \mathcal{O}_{Y^{\prime}}$, where $f^{\prime}: X^{\prime} \rightarrow Y^{\prime}$ is the induced finite flat morphism of usual schemes. Under this isomorphism the pull-back to $Y^{\prime}$ of the map (1.2.4) gets identified with the canonical map $f_{*}^{\prime}\left(f^{\prime}\right) ! \mathcal{O}_{Y^{\prime}} \rightarrow \mathcal{O}_{Y^{\prime}}$. The formation of $f^{!} \mathcal{O}_{Y}$ and of the map (1.2.4) is also compatible with arbitrary base changes $Y^{\prime} \rightarrow Y$ of formal schemes. 
If we define the functor

$$
f^{!}: A_{\mathrm{qc}}(Y) \rightarrow A_{\mathrm{qc}}(X): G \mapsto f^{!} G=f^{!} \mathcal{O}_{Y} \otimes f^{*} G
$$

then for $F \in A_{\mathrm{qct}}(X)$ and $G \in A_{\mathrm{qc}}(Y)$ the composed map

$$
\operatorname{Hom}\left(F, f^{!} G\right) \rightarrow \operatorname{Hom}\left(f_{*} F, f_{*} f^{!} G\right) \rightarrow \operatorname{Hom}\left(f_{*} F, G\right),
$$

where the second arrow is induced by (1.2.4), is an isomorphism.

If $g: Z \rightarrow X$ is another finite flat morphism then $(f g)^{!} \simeq g^{!} \circ f^{!}$on $A_{\mathrm{qc}}(Y)$.

If $i: X^{\prime} \hookrightarrow X$ is a closed embedding of formal schemes such that $f^{\prime}=f \circ$ $i: X^{\prime} \rightarrow Y$ is still flat then we have a natural map

$$
i_{*}\left(f^{\prime}\right)^{!} \mathcal{O}_{Y} \rightarrow f^{!} \mathcal{O}_{Y}
$$

inducing similar canonical maps after an arbitrary base change $Y^{\prime} \rightarrow Y$ with $Y^{\prime}$, a usual scheme. The push-forward of this map by $f_{!}$is compatible with the maps (1.2.4) for $f$ and $f^{\prime}$. For $G \in A_{\mathrm{qc}}(Y)$ the induced map

$$
i_{*}\left(f^{\prime}\right)^{!} G \rightarrow \mathscr{H o m}\left(i_{*} \mathcal{O}_{X^{\prime}}, f^{!} G\right)
$$

is an isomorphism.

(ii) Let $f: X \rightarrow Y$ be a locally nicely ind-finite flat morphism of formal schemes. Then the functor $f_{!}: A_{\mathrm{qct}}(X) \rightarrow A_{\mathrm{qct}}(Y)$ admits an exact right adjoint functor $f^{!}: A_{\mathrm{qct}}(Y) \rightarrow A_{\mathrm{qct}}(X)$.

In the case when $Y$ is a usual scheme so that $\mathcal{O}_{Y} \in A_{\mathrm{qct}}(Y)=\mathrm{Qcoh}(Y)$, the natural map $f ! f^{!} \mathcal{O}_{Y} \rightarrow \mathcal{O}_{Y}$ induces an isomorphism

$$
f^{!} \mathcal{O}_{Y} \otimes f^{*} G \stackrel{\sim}{\longrightarrow} f^{!} G
$$

for $G \in \mathrm{Q} \operatorname{coh}(Y)$.

(iii) Let $f: X \rightarrow Y$ be a (globally) nicely ind-finite morphism of formal schemes. Then there exists a sheaf $f^{!} \mathcal{O}_{Y} \in A_{\overrightarrow{\mathrm{c}}}(X)$ (possibly depending on a nicely ind-finite structure $\left.\left(X_{n}\right)\right)$, flat over $Y$, equipped with a morphism $c_{f}: f_{!}\left(f^{!} \mathcal{O}_{Y}\right) \rightarrow \mathcal{O}_{Y}$, such that we have the induced isomorphism (1.2.7) for $G \in A_{\mathrm{qct}}(Y)$. The formation of $\left(f^{!} \mathcal{O}_{Y}, c_{f}\right)$ is compatible with arbitrary base changes (where the presentation as a limit is obtained by the base change).

Let $g: Z \rightarrow X$ be another nicely ind-finite flat morphism. Then $f g: Z \rightarrow Y$ has a natural nicely ind-finite structure and there is an isomorphism

$$
g^{*}\left(f^{!} \mathcal{O}_{Y}\right) \otimes g^{!} \mathcal{O}_{X} \stackrel{\sim}{\longrightarrow}(f g)^{!} \mathcal{O}_{Y}
$$

and an isomorphism of functors

$$
(f g)^{!} \simeq g^{!} \circ f^{!}: A_{\mathrm{qct}}(Y) \rightarrow A_{\mathrm{qct}}(X) .
$$


Furthermore, for $F \in A_{\mathrm{qct}}(Y)$ the diagram

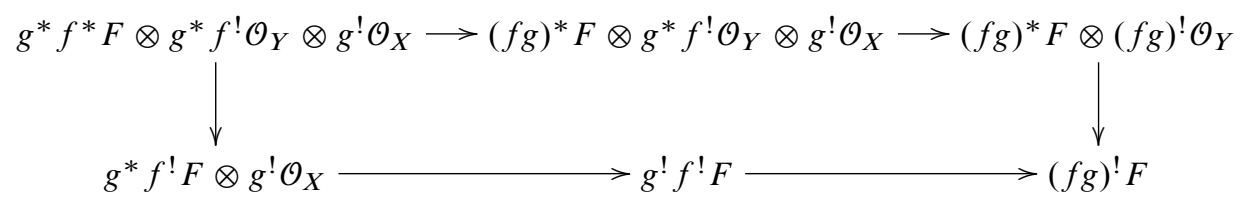

is commutative with arrows induced by (1.2.7), (1.2.8) and (1.2.9).

(iv) Let $f: X \rightarrow Y$ be a locally nicely ind-finite morphism from a formal scheme $X$ to a usual scheme $Y$. Then for quasi-coherent sheaves $F$ on $X$ and $G$ on $Y$ one has a natural isomorphism

$$
\operatorname{Hom}\left(F \otimes f^{!} \mathcal{O}_{Y}, f^{*} G \otimes f^{!} \mathcal{O}_{Y}\right) \simeq \widehat{\operatorname{Hom}}\left(F, f^{*} G\right),
$$

local in $Y($ see (1.2.1)).

Proof. (i) Let us represent $Y$ as the limit of closed subschemes $i_{n}: Y_{n} \hookrightarrow Y$, and set $j_{n}: X_{n}=f^{-1}\left(Y_{n}\right) \hookrightarrow X, f_{n}=\left.f\right|_{X_{n}}: X_{n} \rightarrow Y_{n}$. Note that $X_{n}$ are usual schemes and $X$ is the inductive limit of $X_{n}$. Then every $F \in A_{\text {qct }}(X)$ is the inductive limit of the subsheaves $j_{n *} F_{n}$, where $F_{n}=j_{n}^{*} \mathscr{H o m}\left(j_{n *} \mathcal{O}_{X_{n}}, F\right)$. Note that each $F_{n}$ is quasi-coherent (see [2], Cor. 3.1.6(d)). Also, by Proposition 1.2.2(v), we have $f_{*} F=\lim _{n} i_{n *} f_{n *} F_{n}$. Similarly, for $G \in A_{\mathrm{qc}}(Y)$ let us denote $G_{n}=i_{n}^{*} \mathscr{H o m}\left(i_{n *} \mathcal{O}_{Y_{n}}, G\right) \in A_{\mathrm{qc}}\left(Y_{n}\right)$. Then by Proposition 1.1.5, we have

$$
\begin{aligned}
\operatorname{Hom}\left(f_{*} F, G\right) & =\underset{\lim }{\leftarrow} \operatorname{Hom}\left(f_{n *} F_{n}, G_{n}\right) \\
& \simeq \underset{\lim }{\leftarrow} \operatorname{Hom}\left(F_{n}, f_{n}^{!} G_{n}\right) \\
& \simeq \underset{\lim }{\leftarrow} \operatorname{Hom}\left(F_{n}, f_{n}^{!} \mathcal{O}_{Y_{n}} \otimes f_{n}^{*} G_{n}\right) .
\end{aligned}
$$

Using the compatibility of $f_{n+1}^{!}$with the base change $Y_{n} \rightarrow Y_{n+1}$ (Proposition 1.1.5) we obtain

$$
\left.f_{n+1}^{!} \mathcal{O}_{Y_{n+1}}\right|_{n} \simeq f_{n}^{!} \mathcal{O}_{Y_{n}}
$$

Therefore,

$$
f^{!} \mathcal{O}_{Y}:={\underset{\lim }{n}}_{n} j_{n *} f_{n}^{!} \mathcal{O}_{Y_{n}}
$$

is a coherent sheaf on $X$ equipped with isomorphisms $j_{n}^{*} f^{!} \mathcal{O}_{Y} \simeq f_{n}^{!} \mathcal{O}_{Y_{n}}$. Hence,

$$
\begin{aligned}
\operatorname{Hom}\left(F, f^{!} \mathcal{O}_{Y} \otimes G\right) & \simeq \lim \operatorname{Hom}\left(j_{n *} F_{n}, f^{!} \mathcal{O}_{Y} \otimes f^{*} G\right) \\
& \simeq \lim \operatorname{Hom}\left(F_{n}, j_{n}^{*}\left(f^{!} \mathcal{O}_{Y}\right) \otimes f_{n}^{*} G_{n}\right) \\
& \simeq \underset{\lim }{\leftarrow} \operatorname{Hom}\left(F_{n}, f_{n}^{!} \mathcal{O}_{Y_{n}} \otimes f_{n}^{*} G_{n}\right)
\end{aligned}
$$

which gives a natural isomorphism

$$
\operatorname{Hom}\left(f_{*} F, G\right) \simeq \operatorname{Hom}\left(F, f^{!} G\right) .
$$


Furthermore, we have

$$
f_{*} f^{!} \mathcal{O}_{Y} \simeq \lim _{\leftarrow *} f_{n}^{!} \mathcal{O}_{Y_{n}},
$$

so the canonical maps $f_{n *} f_{n}^{!} \mathcal{O}_{Y_{n}} \rightarrow \mathcal{O}_{Y_{n}}$ give rise to a map (1.2.4). Is is easy to see that the above isomorphism is induced by (1.2.4) (one can replace $F$ by $j_{n *} F_{n}$, so the statement reduces to the similar statement for $f_{n}$ ).

Now let us consider a base change diagram

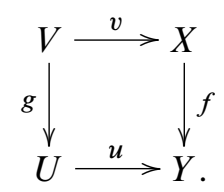

Assume first that $U$ is a usual scheme. Then using the adjoint pair $\left(g_{*}, g^{!}\right)$and the morphism $f_{*} f^{!} \mathcal{O}_{Y} \rightarrow \mathcal{O}_{U}$, as in the proof of Proposition 1.1.5, we construct a natural morphism

$$
v^{*} f^{!} \mathcal{O}_{Y} \rightarrow g^{!} \mathcal{O}_{U}
$$

Since the map $U \rightarrow Y$ locally factors through some subscheme $Y_{n}$ it follows easily from our construction that (1.2.11) is an isomorphism. Next, if $U$ is a formal scheme we can represent it as the $\operatorname{limit} \lim _{n} U_{n}$ of closed subschemes and consider the corresponding closed subschemes $V_{n} \subset V$ so that $V=\lim _{n} V_{n}$, and let $g_{n}: V_{n} \rightarrow U_{n}$ be the induced morphisms. Let also $v_{n}: V_{n} \rightarrow X$ be the morphisms induced by $v: V \rightarrow X$. Then the above argument gives a natural isomorphism $v_{n}^{*} f^{!} \mathcal{O}_{Y} \simeq g_{n}^{!} \mathcal{O}_{U_{n}}$ for each $n$. Passing to inverse limits we get that (1.2.11) is an isomorphism (to represent the left-hand side as a limit we use the fact that $f^{!} \mathcal{O}_{Y}$ is a coherent sheaf on $X$ ).

If $g: Z \rightarrow X$ is another finite flat morphism then to construct an isomorphism of functors $(f \circ g)^{!} \simeq g^{!} \circ f^{!}$is equivalent to constructing an isomorphism

$$
(f \circ g)^{!} \mathcal{O}_{Y} \simeq g^{!} \mathcal{O}_{Y} \otimes g^{*} f^{!} \mathcal{O}_{Y}
$$

of coherent sheaves on $Z$. By choosing a representation $Y=\lim _{n} Y_{n}$ as above and using the definitions this immediately reduces to the case of schemes, where we can use Proposition 1.1.5.

Let $i: X^{\prime} \rightarrow X$ be a closed embedding such that $f^{\prime}=f \circ i: X^{\prime} \rightarrow Y$ is still flat. The map (1.2.5) is obtained by passing to limit from the similar maps associated with the closed embeddings $X^{\prime} \cap X_{n} \rightarrow X_{n}$ and the finite flat maps $f_{n}: X_{n} \rightarrow Y_{n}$. The proof of the fact that (1.2.6) is an isomorphism easily reduces to the case of schemes.

(ii) First, let us replace $Y$ by its affine open subset and $X$ by its connected component so that $X$ is affine and is the inductive limit of a sequence of closed formal subschemes $k_{n}: X_{n} \hookrightarrow X$, where each $X_{n}$ is finite and flat over $Y$. Note that in this situation we have $f_{!}=f_{*}$. Set $f_{n}=f \circ k_{n}: X_{n} \rightarrow Y$. Since $X$ and $X_{n}$ are affine, we have $X=\operatorname{Spf}(A), X_{n}=\operatorname{Spf}\left(A / I_{n}\right)$, where $A=\lim _{n} A / I_{n}$ as a topological 
ring. Therefore, any ideal of definition $J \subset A$ contains $I_{n}$ for some $n$. It follows that for every $F \in A_{\text {qct }}(X)$ we have $F=\lim _{n} \mathscr{H o m}\left(\mathcal{O}_{X} / \mathcal{I}_{n}, F\right)$, where $\mathcal{I}_{n} \subset \mathcal{O}_{X}$ is the ideal sheaf of $X_{n}$. Set $F_{n}=k_{n}^{*} \mathscr{H o m}\left(\mathcal{O}_{X} / \mathcal{I}_{n}, F\right) \in A_{\text {qct }}\left(X_{n}\right)$. As in part (i), we have

$$
\operatorname{Hom}\left(f_{*} F, G\right) \simeq \underset{\lim _{n}}{\operatorname{Hom}}\left(F_{n}, f_{n}^{!} G\right)
$$

Next, by part (i), for $G \in A_{\text {qct }}(Y)$ we have isomorphisms

$$
\left(k_{n, n+1}\right)_{*}\left(f_{n}^{!} G\right) \simeq \mathscr{H o m}\left(\left(k_{n, n+1}\right)_{*} \mathcal{O}_{X_{n}}, f_{n+1}^{!} G\right),
$$

where $k_{n, n+1}: X_{n} \hookrightarrow X_{n+1}$ is the natural embedding. Therefore, we have natural maps $k_{n *} f_{n}^{!} G \rightarrow\left(k_{n+1}\right)_{*} f_{n+1}^{!} G$ so that setting

$$
f^{!} G:=\lim _{n} k_{n *} f_{n}^{!} G
$$

we have $k_{n *} f_{n}^{!} G \simeq \operatorname{Hom}\left(\mathcal{O}_{X} / \mathcal{I}_{n}, f^{!} G\right)$. It follows that

$$
\lim _{n} \operatorname{Hom}\left(F_{n}, f_{n}^{!} G\right) \simeq \underset{\leftarrow}{\lim _{n}} \operatorname{Hom}\left(k_{n *} F_{n}, f^{!} G\right) \simeq \operatorname{Hom}\left(F, f^{!} G\right)
$$

so $G \rightarrow f^{!} G$ is right adjoint to $f_{*}$.

Next, assume that $Y$ is covered by open subsets $Y_{i}$ such that the morphisms $f_{Y_{i}}: X_{i}=f^{-1}\left(Y_{i}\right) \rightarrow Y_{i}$ have the above structure. Then for each $i$ we have the exact functor $f_{Y_{i}}^{!}: A_{\mathrm{qct}}\left(Y_{i}\right) \rightarrow A_{\mathrm{qct}}\left(X_{i}\right)$, right adjoint to $\left(f_{Y_{i}}\right)_{!}$. Furthermore, these functors are compatible with restrictions to open subsets, so we can glue them into an exact functor $f^{!}: A_{\text {qct }}(Y) \rightarrow A_{\text {qct }}(X)$, right adjoint to $f_{!}$.

The isomorphism (1.2.7) in the case when $Y$ is a usual scheme will follow from part (iii).

(iii) Let us again consider the situation from the beginning of the proof of (ii). We have natural maps

$$
\left(k_{n, n+1}\right)_{*}\left(f_{n}^{!} \mathcal{O}_{Y}\right) \rightarrow \mathscr{H o m}\left(\left(k_{n, n+1}\right)_{*} \mathcal{O}_{X_{n}}, f_{n+1}^{!} \mathcal{O}_{Y}\right) \rightarrow f_{n+1}^{!} \mathcal{O}_{Y},
$$

so the sheaves $\left(k_{n *} f_{n}^{!} \mathcal{O}_{Y}\right)$ form an inductive system in $A_{\mathrm{c}}(X)$, and we can set

$$
f^{!} \mathcal{O}_{Y}=\lim _{n} k_{n *} f_{n}^{!} \mathcal{O}_{Y} .
$$

Note that in the case when $Y$ is a usual scheme we have $\mathcal{O}_{Y} \in A_{\text {qct }}(Y)$, and this definition agrees with (1.2.12). Each $k_{n *} f_{n}^{!} \mathcal{O}_{Y}$ is flat over $Y$, hence so is $f^{!} \mathcal{O}_{Y}$. Now using part (i) and (1.2.12) we obtain for $G \in A_{\text {qct }}(Y)$

$$
f^{!} \mathcal{O}_{Y} \otimes f^{*} G \simeq \lim _{n} k_{n *}\left(f_{n}^{!} \mathcal{O}_{Y} \otimes f_{n}^{*} G\right) \simeq \lim _{n} k_{n *} f_{n}^{!} G=f^{!} G
$$

as claimed. Note also that $f_{*} f^{!} \mathcal{O}_{Y}=\lim _{n} f_{n *} f_{n}^{!} \mathcal{O}_{Y}$ and we have a system of compatible morphisms $f_{n *} f_{n}^{!} \mathcal{O}_{Y} \rightarrow \mathcal{O}_{Y}$ (see part (i)), so we get a morphism $f_{*} f^{!} \mathcal{O}_{Y} \rightarrow \mathcal{O}_{Y}$. Hence, by the projection formula, we have a natural map

$$
f_{*}\left(f^{!} \mathcal{O}_{Y} \otimes f^{*} G\right) \simeq f_{*} f^{!} \mathcal{O}_{Y} \otimes G \rightarrow G,
$$


hence, by adjunction, a map (1.2.7). One can easily check that it coincides with the above isomorphism by reducing to the case of a finite morphism.

The fact that the formation of $f^{!} \mathcal{O}_{Y}$ is compatible with any base change $Y^{\prime} \rightarrow Y$ follows from a similar result for finite morphisms (see part (i)).

If $g: Z \rightarrow Y$ is another nicely ind-finite morphism then replacing $Z$ by its connected component we can assume that $Z=\lim _{m} Z_{m}$, where $g_{m}=\left.g\right|_{Z_{m}}: Z_{m} \rightarrow$ $X$ are finite and flat. Let us set $Z_{m, n}=g_{m}^{-1}\left(X_{n}\right) \subset Z_{m}$. Then the induced map $g_{m, n}: Z_{m, n} \rightarrow X_{n}$ is finite and flat, hence, $Z_{m, n}$ is finite and flat over $Y$. Thus, $Z=\lim _{m, n} Z_{m, n}$ is a nicely ind-finite structure on $f g: Z \rightarrow Y$. The isomorphism $(f g)^{!} \simeq g^{!} \circ f^{!}$follows by passing to adjoint functors from $(f g) ! \simeq f ! \circ g_{!}$.

To construct a map (1.2.8), we note that it should correspond by adjunction to a map

$$
(f g)_{!}\left(g^{*} f^{!} \mathcal{O}_{Y} \otimes g^{!} \mathcal{O}_{X}\right) \simeq f_{!}\left(f^{!} \mathcal{O}_{Y} \otimes g_{!} g^{!} \mathcal{O}_{X}\right) \rightarrow \mathcal{O}_{Y},
$$

where we used the projection formula. We clearly have such a map induced by the maps $g_{!} g^{!} \mathcal{O}_{X} \rightarrow \mathcal{O}_{X}$ and $f_{!} f^{!} \mathcal{O}_{Y} \rightarrow \mathcal{O}_{Y}$. To see that it is an isomorphism we apply the definitions (1.2.13) using the nicely ind-finite structures on $f, g$ and $f g$. Namely, let $l_{m}: Z_{m} \rightarrow Z$ and $l_{m, n}: Z_{m, n} \rightarrow Z$ denote the natural closed embeddings. Then we have

$$
\begin{aligned}
g^{*} f^{!} \mathcal{O}_{Y} \otimes g^{!} \mathcal{O}_{X} & \stackrel{(1)}{\simeq} g^{*} f^{!} \mathcal{O}_{Y} \otimes \lim _{m} l_{m *} g_{m}^{!} \mathcal{O}_{X} \\
& \stackrel{(2)}{\simeq} \lim _{m} l_{m *}\left(g_{m}^{*} f^{!} \mathcal{O}_{Y} \otimes g_{m}^{!} \mathcal{O}_{X}\right) \\
& \stackrel{(3)}{\simeq} \lim _{m, n} l_{m, n *}\left(g_{m, n}^{*} f_{n}^{!} \mathcal{O}_{Y} \otimes g_{m, n}^{!} \mathcal{O}_{X}\right) \\
& \stackrel{(4)}{\simeq} \lim _{m, n} l_{m, n *}\left(f_{n} g_{m, n}\right) ! \mathcal{O}_{Y} \\
& \stackrel{(5)}{\simeq}(f g)^{!} \mathcal{O}_{Y},
\end{aligned}
$$

where (1) and (5) are given by (1.2.13), (2) follows from the projection formula, (3) uses (1.2.13) and the compatibility of $g_{m}^{!} \mathcal{O}_{X}$ with the base change (see part (i)), and (4) uses an isomorphism (1.2.8) for finite morphisms that follows from part (i).

The proof of the commutativity of the diagram is tedious but straightforward: since the target vertex is $(f g)^{!} F$, we can use adjunction to rewrite the commutative diagram applying $(f g)_{!}=f ! g_{\text {! }}$ to other vertices.

(iv) It is enough to consider the situation when $X=\lim X_{n}$, where each $X_{n}$ is flat and finite over $Y$. Let us use the same notation as in part (ii). The natural map is obtained as follows: an element $\alpha \in \widehat{\operatorname{Hom}}\left(F, f^{*} G\right)$ induces a map $k_{n}^{*} F \rightarrow f_{n}^{*} G$ for each $n$, hence, we get a compatible system of maps

$$
F \otimes k_{n *} f_{n}^{!} \mathcal{O}_{Y} \rightarrow f^{*} G \otimes k_{n *} f_{n}^{!} \mathcal{O}_{Y}
$$


Taking the inductive limits and using the formula (1.2.13) we get a map $F \otimes f^{!} \mathcal{O}_{Y} \rightarrow$ $f^{*} G \otimes f^{!} \mathcal{O}_{Y}$. To see that it is an isomorphism we use (1.2.7):

$$
\begin{aligned}
\operatorname{Hom}\left(F \otimes f^{!} \mathcal{O}_{Y}, f^{*} G \otimes f^{!} \mathcal{O}_{Y}\right) & \simeq \lim _{\longleftarrow}\left(k_{n}^{*}\left(k_{n}^{*} F \otimes f_{n}^{!} \mathcal{O}_{Y}, f^{!} G\right)\right. \\
& \simeq \lim _{\longleftarrow}\left(k_{n}^{*} F \otimes f_{n}^{!} \mathcal{O}_{Y}, f_{n}^{!} G\right) .
\end{aligned}
$$

By Proposition 1.1.5, this is isomorphic to

$$
\underset{\lim }{\longleftarrow}\left(k_{n}^{*} F, f_{n}^{*} G\right) \simeq \widehat{\operatorname{Hom}}\left(F, f^{*} G\right)
$$

Example. Let $k$ be a field. A formal scheme $X$ is nicely ind-finite over $\operatorname{Spec}(k)$ if and only it is ldu-pseudofinite over $k$, i.e., every connected component of $X$ is of the form $\operatorname{Spf}(A)$, where $A$ is a noetherian adic $k$-algebra, such that $A / J$ is finite-dimensional over $k$ for an ideal of definition $J$. Thus, if $G$ is a formal $k$-group (see the beginning of Section 3 for our conventions on formal $k$-groups) then $G$ is nicely ind-finite over $\operatorname{Spec}(k)$. More generally, if $X$ is a formal scheme over $k$, and $f: E \rightarrow X$ is a $G$-torsor then $f$ is locally nicely ind-finite (this immediately reduces to the case of a trivial $G$-torsor).

Let us point out the following corollary from Proposition 1.2.5. For a formal scheme $X$, locally nicely ind-finite flat over a usual scheme $S$, we set $\omega_{X / S}=\omega_{\pi}:=$ $\pi^{!} \mathcal{O}_{S} \in A_{\mathrm{qct}}(X)$, where $\pi: X \rightarrow S$ is the structure morphism.

Corollary 1.2.6. Let $S$ be a scheme, $X$ and $Y$ formal schemes over $S$, where $Y$ is locally nicely ind-finite flat over $S$. Then for $F \in D_{\mathrm{qct}}(X)$ there is a natural isomorphism on $X \times_{S} Y$

$$
\alpha_{X, Y}: p_{X}^{*} F \otimes p_{Y}^{*} \omega_{Y / S} \stackrel{\sim}{\longrightarrow} p_{X}^{!} F,
$$

where $p_{X}$ and $p_{Y}$ are the projections from $X \times_{S} Y$ to $X$ and $Y$, respectively. Assume that $T$ is another scheme, $Y \rightarrow T$ is a morphism, and $Z$ is a formal scheme, nicely ind-finite flat over $T$. Let us form the fibered product $X \times_{S} Y \times_{T} Z$, and let $p_{X}, p_{Y}$, $p_{X Y}$, etc., denote the projections from this product to the partial products of factors. Then $Y \times_{T} Z$ is locally nicely ind-finite flat over $T$, and the diagram

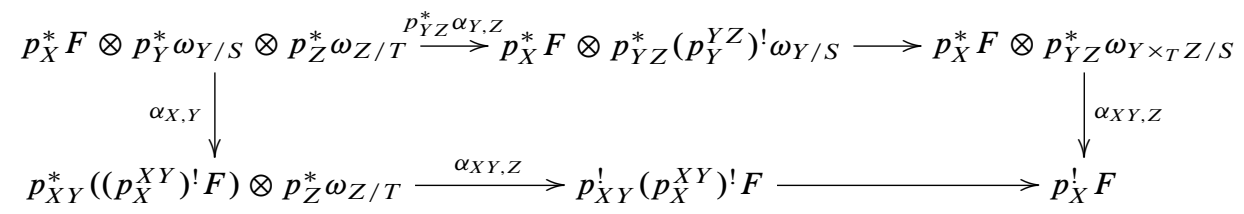

is commutative, where $p_{X}^{X Y}: X \times_{S} Y \rightarrow X$ (resp. $p_{Y}^{Y Z}: Y \times_{T} Z \rightarrow Y$ ) are the natural projections, the marked arrows are induced by (1.2.14), the remaining arrows are obtained from the natural isomorphism $(f g)^{!} \simeq g^{!} \circ f^{!}$. 
Proof. By adjunction, a map (1.2.14) should correspond to morphism on $X$

$$
p_{X !}\left(p_{X}^{*} F \otimes p_{Y}^{*} \omega_{Y / S}\right) \simeq F \otimes p_{X !} p_{Y}^{*} \omega_{Y / S} \rightarrow F
$$

(where we used the projection formula). Applying Theorem 1.2.4(ii), (iii) to the cartesian diagram

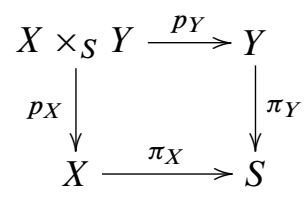

we obtain an isomorphism $F \otimes p_{X !} p_{Y}^{*} \omega_{Y / S} \simeq F \otimes \pi_{X}^{*}\left(\pi_{Y !}\right) \omega_{Y / S}$. Now the desired morphism is induced by the canonical map $\pi_{Y !} \omega_{Y / S}=\pi_{Y !} \pi_{Y}^{!} \mathcal{O}_{S} \rightarrow \mathcal{O}_{S}$. To check that (1.2.14) is an isomorphism we can argue locally in $S$, so we can assume that $\pi_{Y}: Y \rightarrow S$ is (globally) nicely ind-finite. Then we can apply the compatibility of the formation of $\omega_{Y / S}=\pi_{Y}^{!} \mathcal{O}_{S}$ with the base change, which gives an isomorphism $p_{Y}^{*} \omega_{Y / S} \simeq p_{X}^{!} \mathcal{O}_{X}$. Now (1.2.14) follows from (1.2.7).

In the case when $Z$ is nicely ind-finite over $T$, then the projection $Y \times_{T} Z \rightarrow S$ is the composition of $p_{Y}^{Y Z}$ with $\pi_{Y}$, so it is nicely ind-finite. The desired commutativity of the diagram follows from the commutativity of the diagram in Proposition 1.2.5 (iii) applied to the composition of $p_{X}^{X Y}$ with $p_{X Y}$ (note that both these projections are nicely ind-finite).

1.3. Biextensions. Let $\delta$ be a site, and let $\operatorname{sh}(S)$ denote the category of sheaves of abelian groups over $\delta$. We refer to sec. 1.4 of [18] for basic definitions concerning (strictly commutative) Picard stacks over $S$. Roughly speaking, these are sheaves of categories $\mathcal{P}$ over $S$ equipped with a commutative group law $\mathcal{P} \times \mathcal{P} \rightarrow \mathcal{P}$ satisfying appropriate axioms. If $K=\left[K^{-1} \stackrel{d}{\rightarrow} K^{0}\right]$ is a complex over $S h(\mathcal{S})$, concentrated in degrees -1 and 0 , then one has the corresponding Picard stack $\operatorname{ch}(K)$ with objects given by sections of $K^{0}$, where a morphism between $x$ and $y$ in $K^{0}(U)$ is an element $f \in K^{-1}(U)$ such that $d f=y-x$. In fact, every Picard stack can be represented in this way (see [18], 1.4.13). For example, if $G$ is a sheaf of abelian groups then the Picard stack of $G$-torsors is equivalent to $\operatorname{ch}(G[1])$ (see [18], 1.4.21).

For a pair of Picard stacks $\mathcal{P}_{1}, \mathcal{P}_{2}$ there is a natural Picard stack $\operatorname{HOM}\left(\mathcal{P}_{1}, \mathcal{P}_{2}\right)$ of homofunctors $\mathcal{P}_{1} \rightarrow \mathcal{P}_{2}$ (the corresponding term in [18], 1.4 is "foncteur additif"). For complexes of sheaves $K_{1}$ and $K_{2}$, concentrated in degrees -1 and 0 , one has

$$
\operatorname{HOM}\left(\operatorname{ch}\left(K_{1}\right), \operatorname{ch}\left(K_{2}\right)\right) \simeq \operatorname{ch} \tau_{\leq 0} R \underline{\operatorname{Hom}}\left(K_{1}, K_{2}\right),
$$

where on the right-hand side we view $K_{1}$ and $K_{2}$ as objects of the derived category $D^{b}(\operatorname{Sh}(\mathcal{S}))$ (see [18], (1.4.18.1)).

Lemma 1.3.1 ([18], 1.4.23). Let $K=\left[K^{-1} \rightarrow K^{0}\right]$ be a complex over $S h(\S)$, and let $G \in S h(S)$. Then the Picard stack of extensions $\operatorname{EXT}(K, G)$ (i.e., of extensions of $K^{0}$ by $G$, trivialized over $\left.K^{-1}\right)$ is equivalent to $\operatorname{HOM}(\operatorname{ch}(K), \operatorname{ch}(G[1]))$. 
For a triple $\mathcal{P}_{1}, \mathcal{P}_{2}, \mathcal{P}_{3}$ of Picard stacks there is a Picard stack $\operatorname{HOM}\left(\mathcal{P}_{1}, \mathcal{P}_{2} ; \mathcal{P}_{3}\right)$ of biadditive functors $\mathcal{P}_{1} \times \mathcal{P}_{2} \rightarrow \mathcal{P}_{3}$ (see [18], 1.4.8). In terms of complexes of sheaves $K_{1}, K_{2}, K_{3}$ as above, we have

$$
\begin{aligned}
\operatorname{HOM}\left(\operatorname{ch}\left(K_{1}\right), \operatorname{ch}\left(K_{2}\right) ; \operatorname{ch}\left(K_{3}\right)\right) & \simeq \operatorname{ch} \tau_{\leq 0} R \underline{\operatorname{Hom}}\left(\tau_{\geq-1}\left(K_{1} \otimes^{\mathbb{L}} K_{2}\right), K_{3}\right) \\
& \simeq \operatorname{ch} \tau_{\leq 0} R \underline{\operatorname{Hom}}\left(K_{1} \otimes^{\mathbb{L}} K_{2}, K_{3}\right)
\end{aligned}
$$

(using [18], (1.4.8.1), 1.4.20 and (1.3.1)).

Lemma 1.3.2. One has a natural equivalence

$$
\operatorname{HOM}\left(\mathcal{P}_{1}, \mathcal{P}_{2} ; \mathcal{P}_{3}\right) \simeq \operatorname{HOM}\left(\mathcal{P}_{1}, \operatorname{HOM}\left(\mathcal{P}_{2}, \mathcal{P}_{3}\right)\right)
$$

Proof. This can be deduced directly from the definitions. Alternatively, we can realize each Picard stack $\mathcal{P}_{i}$ as $\operatorname{ch}\left(K_{i}\right)$ for some complexes $K_{i}(i=1,2,3)$ concentrated in degrees -1 and 0 . Using (1.3.1) and (1.3.2) we obtain similar representations for both sides of (1.3.3). It remains to use the isomorphism

$$
\begin{aligned}
R \underline{\operatorname{Hom}}\left(K_{1} \otimes^{\mathbb{L}} K_{2}, K_{3}\right) & \simeq R \underline{\operatorname{Hom}}\left(K_{1}, R \underline{\operatorname{Hom}}\left(K_{2}, K_{3}\right)\right) \\
& \simeq R \underline{\operatorname{Hom}}\left(K_{1}, \tau_{\leq 0} R \underline{\operatorname{Hom}}\left(K_{2}, K_{3}\right)\right) .
\end{aligned}
$$

Proposition 1.3.3. Let $P, Q$ and $G$ be three sheaves of commutative groups on some site. Then the category of biextensions of $P \times Q$ by $G$ is equivalent to the category of homofunctors $P \rightarrow \operatorname{EXT}(Q, G)$.

Proof. Let BIEXT $(P, Q ; G)$ denote the Picard stack of biextensions of $P \times Q$ by $G$. Then we have a natural functor

$$
\operatorname{BIEXT}(P, Q ; G) \rightarrow \operatorname{HOM}(\operatorname{ch}(P), \operatorname{ch}(Q) ; \operatorname{ch}(G[1])),
$$

which associates with a biextension the corresponding map from pairs of sections $(p, q)$ of $P \times Q$ to $G$-torsors. Using (1.3.3) and Lemma 1.3.1 we see that the righthand side is equivalent to the Picard stack of homofunctors from $P$ to $\operatorname{EXT}(Q, G)$. Thus, it suffices to prove that (1.3.4) is an equivalence. This can be checked directly using the definition of the biextension as in [28], 2.0. Here is a different way to check this. By (1.3.2), we have

$$
\operatorname{HOM}(\operatorname{ch}(P), \operatorname{ch}(Q) ; \operatorname{ch}(G[1])) \simeq \operatorname{ch} \tau_{\leq 0} R \underline{\operatorname{Hom}}\left(P \otimes^{\mathbb{L}} Q, G[1]\right) .
$$

Therefore, the sheaf of automorphisms of the neutral object of this Picard stack is $\operatorname{Hom}\left(P \otimes^{\mathbb{Q}} Q, G\right)$ while the sheaf associated with the isomorphism classes of objects is $\underline{\operatorname{Ext}}^{1}(P \otimes \mathbb{L} Q, G)$. But these are exactly the same sheaves as one gets for the Picard stack of biextensions (see [28], 2.5.4 and 3.6.5).

The following result follows immediately from [29], (1.1.6) and 1.5 (it can also be derived from the above Proposition by considering the subcategory of locally trivial extensions in $\operatorname{EXT}(Q, G))$. 
Proposition 1.3.4. For $P, Q, G$ as above there is a fully faithful functor

$$
\operatorname{EXT}(P, \underline{\operatorname{Hom}}(Q, G)) \rightarrow \operatorname{BIEXT}(P, Q ; G) .
$$

If $\operatorname{Ext}^{1}(Q, G)=0$ then this functor is an equivalence.

The condition $\underline{\operatorname{Ext}}^{1}(Q, G)=0$ is satisfied in the following important case.

Lemma 1.3.5 ([29], 3.3.1). Let $G$ be a finite flat group scheme over $S$. Then $\underline{\operatorname{Ext}}^{1}\left(G, \mathbb{G}_{m}\right)=0$.

Now let $k$ be a field of characteristic zero. Then similar vanishing holds for affine commutative algebraic groups over $k$ and for formal $k$-groups (for our assumptions on formal $k$-groups see the beginning of Section 3).

Lemma 1.3.6. (i) Let $G$ be an affine commutative algebraic group over $k$ or a formal $k$-group. Then $\operatorname{Ext}^{1}\left(G, \mathbb{G}_{m}\right)=0$ (with respect to flat topology).

(ii) If $0 \rightarrow G_{1} \rightarrow G_{2} \rightarrow G_{3} \rightarrow 0$ is an exact sequence of formal $k$-groups then there exists a finite field extension $k \subset k^{\prime}$ and a section $\left(G_{3}\right)_{k^{\prime}} \rightarrow\left(G_{2}\right)_{k^{\prime}}$ (not required to be a group homomorphism). Hence, $G_{2} \rightarrow G_{3}$ is a $G_{1}$-torsor in flat topology.

Proof. (i) For affine commutative algebraic groups this follows immediately from the Cartier duality since $\mathbb{G}_{m}$ is dual to $\mathbb{Z}$. Now assume $G$ is a formal $k$-group. Let $G^{0}$ be the component of identity in $G$. Passing to a finite extension of $k$ we can assume that $G / G^{0}$ is a finitely generated discrete abelian group, so $\underline{\operatorname{Ext}}^{1}\left(G / G^{0}, \mathbb{G}_{m}\right)=0$. Now if we have a group extension

$$
1 \rightarrow \mathbb{G}_{m} \rightarrow H \rightarrow G^{0} \rightarrow 1
$$

then we can pass to the corresponding extension of Lie algebras

$$
0 \rightarrow k \rightarrow L H \rightarrow L G^{0} \rightarrow 0 .
$$

Since the characteristic of $k$ is zero and all the Lie brackets here are trivial, we have a splitting $L G^{0} \rightarrow L H$ that induces a splitting $G^{0} \rightarrow H$

(ii) Since all our formal $k$-groups are assumed to be commutative, the extension splits over $G_{3}^{0}$ (by considering the extension of Lie algebras). Thus, it is enough to consider the case when $G_{3}$ is étale. Passing to a finite extension of $k$ we can assume that $G_{3}$ is a finitely generated discrete abelian group, in which case the statement is clear.

Remark. The vanishing in Lemma 1.3.6 (i) also holds for formal groups over fields of finite characteristic - one can reduce this to the case of finite group schemes. 


\section{Kernel algebras}

In this section we present a formalism generalizing the techniques of Fourier-Mukai transforms for $D$-algebras developed in [44]. We consider more general "nonlocal" algebras and generalize the main construction of the "circle product" from [44] by making use of appropriate resolutions. The main result of this section is Theorem 2.5.1 generalizing Thm. 6.5 of [44]. Throughout this section we work over a fixed base scheme $S$, and all our schemes are assumed to be $S$-schemes. We denote simply by $X \times Y$ the fibered product over $S$. All schemes in this section are assumed to be semi-separated.

2.1. Convolution for quasi-coherent sheaves. Let us start by recalling the basic properties of the convolution operation for quasi-coherent sheaves (called the circle product in [44]). Given $F \in D_{\mathrm{qc}}(X \times Y)$ and $G \in D_{\mathrm{qc}}(Y \times Z)$ we set

$$
F \circ_{Y} G=p_{13 *}\left(p_{12}^{*} F \otimes p_{23}^{*} G\right),
$$

where $p_{i j}$ are the projections from $X \times Y \times Z$ to the double products of factors.

Lemma 2.1.1. (i) Assume that in each pair of $S$-schemes $(X, Z)$ and $(Y, T)$ at least one is flat over $S$. Then for $F \in D_{\mathrm{qc}}(X \times Y), G \in D_{\mathrm{qc}}(Y \times Z)$ and $H \in D_{\mathrm{qc}}(Z \times T)$ one has

$$
\left(F \circ_{Y} G\right) \circ_{Z} H \simeq F \circ_{Y}\left(G \circ_{Z} H\right) .
$$

(ii) Assume that $X$ is flat over $S$. Then for $F \in D_{\mathrm{qc}}(X \times Y)$ and $G \in D_{\mathrm{qc}}(Y)$ one has

$$
F \circ_{Y} \Delta_{*} G \simeq F \otimes p_{2}^{*} G .
$$

The proof is a simple application of the projection and base change formulae (cf. [38], Prop. 1.3).

Lemma 2.1.2. (i) Assume that we are given a morphism $g: Y \rightarrow Y^{\prime}$ and let $X$ and $Z$ be schemes. If $g$ is flat or $Z$ is flat over $S$ then for $F \in D_{\mathrm{qc}}(X \times Y)$ and $G^{\prime} \in D_{\mathrm{qc}}\left(Y^{\prime} \times Z\right)$ we have a natural isomorphism

$$
\left(\left(\operatorname{id}_{X} \times g\right)_{*} F\right) \circ_{Y^{\prime}} G \simeq F \circ_{Y}\left(g \times \operatorname{id}_{Z}\right)^{*} G .
$$

Similarly, ifg isflat or $X$ is flat over $S$ then for $F^{\prime} \in D_{\mathrm{qc}}\left(X \times Y^{\prime}\right)$ and $G \in D_{\mathrm{qc}}(Y \times Z)$ one has

$$
F^{\prime} \circ_{Y^{\prime}}\left(g \times \operatorname{id}_{Z}\right)_{*} G \simeq\left(\left(\operatorname{id}_{X} \times g\right)^{*} F^{\prime}\right){ }^{\circ} G .
$$

(ii) Assume that we are given morphisms $f: X \rightarrow X^{\prime}$ and $h: Z \rightarrow Z^{\prime}$. If $f$ is flat or $Z$ is flat over $S$ then for $F \in D_{\mathrm{qc}}(X \times Y)$ and $G \in D_{\mathrm{qc}}(Y \times Z)$ we have a natural isomorphism

$$
\left(f \times \operatorname{id}_{Z}\right)_{*}\left(F \circ \circ_{Y} G\right) \simeq\left(\left(f \times \operatorname{id}_{Y}\right)_{*} F\right) \circ_{Y} G .
$$


Similarly, if $h$ is flat or $X$ is flat over $S$ then one has

$$
\left(\operatorname{id}_{X} \times h\right)_{*}\left(F \circ_{Y} G\right) \simeq F \circ_{Y}\left(\operatorname{id}_{Y} \times h\right)_{*} G .
$$

(iii) Assume that we are given morphisms $f: X \rightarrow X^{\prime}$ and $h: Z \rightarrow Z^{\prime}$, and let $Y$ be a scheme. Assume that either $f$ and $h$ are flat or $Y$ is flat over $S$. Then for $F \in D_{\mathrm{qc}}\left(X^{\prime} \times Y\right)$ and $G \in D_{\mathrm{qc}}\left(Y \times Z^{\prime}\right)$ one has a natural isomorphism

$$
(f \times h)^{*}\left(F \circ_{Y} G\right) \simeq\left(\left(f \times \operatorname{id}_{Y}\right)^{*} F\right){ }^{\circ} Y\left(\left(\operatorname{id}_{Y} \times h\right)^{*} G^{\prime}\right) .
$$

(iv) Assume that we are given morphisms $f: X \rightarrow X^{\prime}, g: Y \rightarrow Y^{\prime}$, and let $Z$ be a scheme. Assume that either $f$ and $g$ are flat or $Z$ is flat over $S$. Then for $F \in D_{\mathrm{qc}}(X \times Y)$ and $G \in D_{\mathrm{qc}}\left(Y^{\prime} \times Z\right)$ one has a natural isomorphism

$$
\left((f \times g)_{*} F\right) \circ_{Y^{\prime}} G \simeq\left(f \times \operatorname{id}_{Z}\right)_{*}\left(F \circ_{Y}\left(g \times \operatorname{id}_{Z}\right)^{*} G\right) .
$$

Proof. The proofs of (i), (ii) and (iii) are easy applications of the projection and base change formulae. (iv) follows from (i) and (ii).

Assume we are given a triple of morphisms $f: X \rightarrow X^{\prime}, g: Y \rightarrow Y^{\prime}, h: Z \rightarrow$ $Z^{\prime}$, where either all these morphisms are flat, or $X$ and $Z^{\prime}$ are flat over $S$, or $X^{\prime}$ and $Z$ are flat over $S$. Then we can define for $F \in D_{\mathrm{qc}}(X \times Y)$ and $G \in D_{\mathrm{qc}}(Y \times Z)$ a natural morphism

$$
(f \times g)_{*} F \circ_{Y^{\prime}}(g \times h)_{*} G \rightarrow(f \times h)_{*}\left(F \circ \circ_{Y} G\right) .
$$

Indeed, say, assume that $X$ and $Z^{\prime}$ are flat over $S$. Then using (2.1.2) we get an isomorphism

$$
(f \times g)_{*} F \circ_{Y^{\prime}}(g \times h)_{*} G \simeq\left(f \times \operatorname{id}_{Y}\right)_{*}\left(F \circ_{Y}\left(g \times \operatorname{id}_{Z}\right)^{*}(g \times h)_{*} G\right) .
$$

Now using the adjunction morphism

$$
\left(g \times \operatorname{id}_{Z}\right)^{*}(g \times h)_{*} G \simeq\left(g \times \operatorname{id}_{Z}\right)^{*}\left(g \times \operatorname{id}_{Z}\right)_{*}\left(\operatorname{id}_{Y} \times h\right)_{*} G \rightarrow\left(\operatorname{id}_{Y} \times h\right)_{*} G
$$

we get a morphism from $(f \times g)_{*} F \circ_{Y^{\prime}}(g \times h)_{*} G$ to

$$
\left(f \times \operatorname{id}_{Y}\right)_{*}\left(F \circ_{Y}\left(\operatorname{id}_{Y} \times h\right)_{*} G\right) \simeq(f \times h)_{*}\left(F \circ_{Y} G\right),
$$

where the last isomorphism follows from Lemma 2.1.2 (ii). We leave for the reader to formulate and prove the associativity of this construction, say, under the assumption that all schemes involved are flat over $S$.

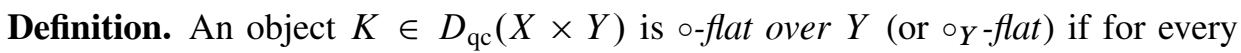
quasi-coherent sheaf $F$ on $Y$ the object $K{ }^{\circ} Y F \in D_{\mathrm{qc}}(X)$ is a sheaf. In the case $X=S$ we have $K \in D_{\mathrm{qc}}(Y)$ and the condition of ${ }^{\circ} Y$-flatness means that the functor $F \mapsto \pi_{*}(F \otimes K)$ on $D_{\mathrm{qc}}$ is $t$-exact, where $\pi: Y \rightarrow S$ is the projection. 
Note that the condition of o-flatness over $Y$ is local over $X$.

Lemma 2.1.3. (i) Let $f: X \rightarrow X^{\prime}$ and $\tilde{g}: \tilde{Y} \rightarrow Y$ be affine morphisms and let $\tilde{f}: \tilde{X} \rightarrow X$ and $g: Y \rightarrow Y^{\prime}$ be flat morphisms. Then $(f \times g)_{*}$ sends ${ }^{\circ} Y$-flat objects to ${ }^{\circ} Y^{\prime}$-flat objects. Assume in addition that either $\tilde{g}$ is flat or $\tilde{X}$ is flat over $S$. Then $(\tilde{f} \times \tilde{g})^{*}$ sends ${ }^{\circ} Y$-flat objects to ${ }^{\circ} \tilde{Y}$-flat objects.

(ii) If $K \in D_{\mathrm{qc}}(X \times Y)$ is ${ }^{\circ} Y$-flat then $K$ is a quasi-coherent sheaf on $X \times Y$, flat over $Y$. If $Y$ is affine over $S$ then the converse is also true.

(iii) Let $K$ be $a{ }^{\circ} Y$-flat quasi-coherent sheaf on $X \times Y$, and let $Z$ be a scheme. Assume that either $X$ or $Z$ is flat over $S$. Then for any quasi-coherent sheaf $L$ on $Y \times Z$ the object $K{ }^{\circ} L \in D_{\mathrm{qc}}(X \times Z)$ is a sheaf.

(iv) Let $K \in \mathrm{Q} \operatorname{coh}(X \times Y)$ be ${ }^{\circ} Y$-flat and $L \in \mathrm{Q} \operatorname{coh}(Y \times Z)$ be ${ }^{\circ} Z$-flat. Assume that either $X$ or $Z$ is flat over $S$. Then $K{ }^{\circ} Y L \in D_{\mathrm{qc}}(X \times Z)$ is also ${ }^{\circ} Z$-flat.

(v) Assume again that either $X$ or $Z$ is flat over $S$. If $K \in \mathrm{Q} \operatorname{coh}(X \times Y)$ is ${ }^{\circ} Y$-flat and $L$ is a quasi-coherent sheaf on $Y \times Z$, flat over $Z$, then $K{ }^{\circ} Y L \in \mathrm{Q} \operatorname{coh}(X \times Z)$ is also flat over $Z$.

(vi) Let $q: K_{1}^{\bullet} \rightarrow K_{2}^{\bullet}$ is a quasi-isomorphism of bounded complexes of quasicoherent sheaves on $X \times Y$ with ${ }^{\circ} Y$-flat terms. Then for every $L \in \mathrm{Q} \operatorname{coh}(Y \times Z)$ the induced morphism of complexes $K_{1}^{{ }^{\circ}}{ }^{\circ} Y L \rightarrow K_{2}^{\bullet}{ }^{\circ} Y L$ is a quasi-isomorphism.

Proof. (i) This follows from the isomorphisms of Lemma 2.1.2.

(ii) Without loss of generality we can assume $S$ to be affine. Assume first that $Y$ is also affine. Then the functor $p_{1 *}: D_{\mathrm{qc}}(X \times Y) \rightarrow D_{\mathrm{qc}}(X)$ is $t$-exact and conservative. Hence, the condition of ${ }^{\circ} Y$-flatness is equivalent to the condition that the functor $F \mapsto K \otimes p_{2}^{*} F: D_{\mathrm{qc}}(Y) \rightarrow D_{\mathrm{qc}}(X \times Y)$ is $t$-exact. Taking $F=\mathcal{O}_{Y}$ we immediately see that $K$ itself is a sheaf. Furthermore, this exactness condition is easily seen to be equivalent to flatness of $K$ over $Y$.

Now for arbitrary $Y$, using part (i), we see that $\left.K\right|_{X \times U}$ is ${ }^{\circ} U$-flat for any open affine subset $U \subset Y$. As we have just shown this implies that $\left.K\right|_{X \times U}$ is a sheaf, flat over $U$. Hence, $K$ itself is a sheaf, flat over $Y$.

(iii) The problem is local in $Z$, so we can assume that $Z$ is affine. Then it is enough to check that $p_{X *}^{X Z}\left(K{ }^{*}{ }_{Y} L\right) \in D_{\mathrm{qc}}(X)$ is a sheaf, where $p_{X}^{X Z}: X \times Z \rightarrow X$ is the projection. Since $X$ is flat over $S$, by Lemma 2.1.2, one has

$$
p_{X *}^{X Z}\left(K \circ_{Y} L\right) \simeq K \circ_{Y}\left(p_{Y *}^{Y Z} L\right)
$$

where $p^{Y Z}: Y \times Z \rightarrow Y$ is the projection. Now we observe that $p_{Y *}^{Y Z} L$ is a sheaf on $Y$ and use the fact that $K$ is ${ }^{\circ} Y$-flat.

(iv) This follows from the associativity of the convolution in this case (see Lemma 2.1.1 (i)).

(v) Without loss of generality we can assume $S$ and $Z$ to be affine. Now the statement follows from (ii) and (iv).

(vi) It is enough to check that if a bounded complex $K^{\bullet}$ of quasi-coherent sheaves on $X \times Y$ with ${ }^{\circ} Y$-flat terms represents an object $F \in D_{\mathrm{qc}}(X \times Y)$ then the complex 
$K^{\bullet} \circ_{Y} L$ represents $F \circ_{Y} L \in D_{\mathrm{qc}}(X \times Z)$. This can be easily proved by induction in the length of $K^{\circ}$.

Lemma 2.1.4. (i) Let $f: Z \rightarrow X$ be an affine morphism and $g: Z \rightarrow Y$ a morphism of schemes. Then for any quasi-coherent sheaf $F$ on $Z$, flat over $Y$, the quasi-coherent sheaf $(f, g)_{*} F$ on $X \times Y$ is o-flat over $Y$.

(ii) The same assertion is true for the sheaf $(f, g)_{\sigma *} F$ if $X$ and $Y$ are schemes, $Z$ is a formal scheme, $f$ is ldu-affine, and $F$ is a sheaf in $A_{\mathrm{qct}}(Z)$, flat over $Y$.

Proof. (i) The fact that $F$ is flat over $Y$ implies that the functor

$$
D_{\mathrm{qc}}(Y) \rightarrow D_{\mathrm{qc}}(Z): G \mapsto F \otimes g^{*} G
$$

is $t$-exact. Now the assertion follows from the isomorphism

$$
(f, g)_{*} F \circ_{Y} G \simeq f_{*}\left(F \otimes g^{*} G\right)
$$

for $G \in \operatorname{Qcoh}(Y)$ (that follows from the projection formula for the map $(f, g)$ ).

(ii) One can repeat the above argument using the projection formula for sheaves on formal schemes (see Theorem 1.2.3) and the fact that $f_{\sigma *}$ is exact for ldu-affine $f$.

Lemma 2.1.5. Let $X, Y$ and $Z$ be schemes, where either $X$ or $Z$ is flat over $S$.

(i) Let $K$ be $a \circ_{Y}$-flat quasi-coherent sheaf on $X \times Y$. Then the functor

$$
\mathrm{Q} \operatorname{coh}(Y \times Z) \rightarrow \mathrm{Q} \operatorname{coh}(X \times Z): F \mapsto K \circ_{Y} F
$$

commutes with inductive limits.

(ii) Let $\left(K_{i}\right)$ be an inductive system of ${ }^{\circ} Y$-flat quasi-coherent sheaves on $X \times Y$. Then $\lim _{i} K_{i}$ is still $\circ_{Y}$-flat, and for every quasi-coherent sheaf $F$ on $Y \times Z$ the natural map

$$
\lim \left(K_{i}{ }^{\circ} Y F\right) \rightarrow\left(\lim K_{i}\right){ }^{\circ} Y F
$$

is an isomorphism.

Proof. (i) By definition, $K{ }^{\circ} F=p_{X Z *}\left(p_{X Y}^{*} K \otimes p_{Y Z}^{*} F\right)$, where we use projections from $X \times Y \times Z$ to double products of factors. Recall that $K{ }^{\circ} Y F$ is a sheaf by Lemma 2.1.3 (iii), and $K$ is flat over $Y$ by Lemma 2.1.3 (ii). It follows that the sheaf $p_{X Y}^{*} K$ is flat over $Y \times Z$, so we can use the underived functors in the above formula for $K \circ_{Y} F$. All of them commute with inductive limits (for the push-forward see Lemma 1.1.1).

(ii) Note that $\lim K_{i}$ is flat over $Y$, so for $F \in \mathrm{Q} \operatorname{coh}(Y \times Z)$ we have

$$
p_{X Y}^{*}\left(\lim K_{i}\right) \otimes F \simeq\left(\lim p_{X Y}^{*} K_{i}\right) \otimes F \simeq \lim \left(p_{X Y}^{*} K_{i} \otimes F\right),
$$

where we can use the underived functors. Now using Lemma 1.1.1 we find that the derived push-forward of this sheaf by $p_{X Z}$ is concentrated in degree 0 and is isomorphic to $\lim K_{i}{ }^{\circ} Y F$. 
2.2. Kernel algebras. Let $X$ be a flat scheme over $S$. We denote by $\Delta: X \rightarrow X \times X$ the (relative over $S$ ) diagonal embedding. Note that this is an affine morphism, since $X$ and $S$ are semi-separated.

Definition. (i) A kernel algebra over $X$ is an object $\mathcal{A} \in D_{\mathrm{qc}}(X \times X)$ equipped with a morphism $\mu: \mathcal{A}^{\circ} X \mathcal{A} \rightarrow \mathcal{A}$ (product) and a morphism $u: \Delta_{*} \mathcal{O}_{X} \rightarrow \mathcal{A}$ (unit), subject to the usual associativity and unit axioms. Note that in these axioms we use the isomorphisms

$$
\left(\mathcal{A} \circ \circ_{X} \mathcal{A}\right) \circ_{X} \mathcal{A} \simeq \mathcal{A} \circ_{X}\left(\mathcal{A} \circ \circ_{X} \mathcal{A}\right) \text { and } \mathcal{A} \circ_{X} \Delta_{*} \mathcal{O}_{X} \simeq \Delta_{*} \mathcal{O}_{X} \circ X \mathcal{A} \simeq \mathcal{A}
$$

(see Lemma 2.1.1). Homomorphisms between kernel algebras over the same scheme $X$ are defined in an obvious way. A kernel algebra $\mathcal{A}$ over $X$ is called pure if $\mathcal{A}$ is a quasi-coherent sheaf on $X \times X$, o-flat over $X$ with respect to both projections.

(ii) A left (resp. right) module over a pure kernel algebra $\mathcal{A}$ is a quasi-coherent sheaf $F$ on $X$ equipped with a morphism $\mathcal{A}^{\circ}{ }_{X} F \rightarrow F$ (resp. $F{ }^{\circ} X \mathcal{A} \rightarrow F$ ) satisfying the usual associativity and unitality axioms. We denote by $\mathcal{A}$-mod the category of left $\mathcal{A}$-modules.

(iii) We say that a pure kernel algebra $\mathcal{A}$ over $X$ is finite (on the left) if $\mathcal{A}$ is a coherent sheaf on $X \times X$, and the support of $\mathcal{A}$ is proper over $X$ with respect to the first projection. In this case a (left) module $F$ over $\mathcal{A}$ is called coherent if $F$ is a coherent sheaf on $X$. We denote by $\mathcal{A}$ - $\bmod ^{c}$ the category of coherent $\mathscr{A}$-modules.

Henceforward, whenever we consider kernel algebras over $X$ we implicitly assume $X$ to be flat over $S$.

Note that if $\mathcal{A}$ is a pure kernel algebra then by Lemma 2.1.3 (ii), the object $\mathcal{A}^{\circ} X \mathcal{A} \in$ $D_{\mathrm{qc}}(X \times X)$ (resp. $\left.\mathcal{A} \circ_{X} F, F \circ_{X} \mathcal{A}\right)$ appearing in the above definition is a sheaf. Using exactness of the functor $F \mapsto \mathcal{A}_{X} F$ one can immediately check that $\mathcal{A}$-mod (resp. $\mathcal{A}-\bmod ^{\mathrm{c}}$ if $\mathcal{A}$ is finite) is an abelian category.

Lemma 2.2.1. Let $\mathcal{A}$ be a pure kernel algebra over $X$. Then for any inductive system of left A-A-modules $\left(F_{i}\right)$ there is a natural A-module structure on the quasi-coherent sheaf $F=\lim _{i} F_{i}$. The obtained $\mathcal{A}$-module $F$ is the limit of $\left(F_{i}\right)$ in the category of A-modules.

Proof. For each map $F_{i} \rightarrow F_{j}$ in this inductive system there is an induced map of quasi-coherent sheaves $\mathcal{A}^{\circ} \circ_{X} F_{i} \rightarrow \mathcal{A}^{\circ} \circ_{X} F_{j}$, so we get a map of inductive systems $\left(\mathcal{A} \circ{ }_{X} F_{i}\right) \rightarrow\left(F_{i}\right)$. Passing to the limit and using Lemma 2.1.5 (i) we get a map

$$
\mathcal{A} \circ_{X} \lim F_{i} \simeq \lim \mathcal{A}^{\circ} \circ_{X} F_{i} \rightarrow \lim F_{i},
$$

so we get an action of $\mathcal{A}$ on $F=\lim F_{i}$. It is easy to check that this is a structure of an $\mathcal{A}$-module on $F$, and that it is the limit of $\left(F_{i}\right)$ in the category of $\mathcal{A}$-modules. 
Lemma 2.2.2. Let $\mathcal{A}$ be a pure kernel algebra over $X, F$ a left $\mathcal{A}$-module. Then the complex of left A-modules

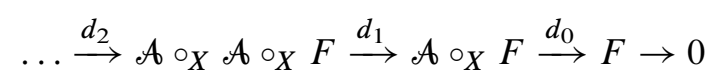

is exact, where the differentials $d_{i}$ are alternating sums of the appropriate operations in two consecutive factors.

Proof. Note that in the case when $X$ is a point this complex is the bar complex of a module over an associative algebra. Generalizing the construction of the standard contracting homotopy from this case, we can consider the map $s_{n}: \mathcal{A}^{\circ} X^{n}{ }^{\circ} X$ $\mathcal{A}^{\circ} X(n+1){ }^{\circ} X F$ induced by the unit morphism $u: \Delta_{*} \mathcal{O}_{X} \rightarrow \mathcal{A}$ in the first factor. One immediately checks that $\left(s_{n}\right)$ is a contracting homotopy for our complex, hence it is acyclic.

Lemma 2.2.3. Let A be a finite pure kernel algebra over $X$. Then the natural functor $D^{-}\left(\mathcal{A}\right.$-mod $\left.{ }^{\mathfrak{c}}\right) \rightarrow D^{-}(\mathcal{A}$-mod $)$ is fully faithful, and its essential image consists of all complexes of A-modules with bounded above coherent cohomology.

Proof. This is proved in the same way as for usual sheaves of $\mathcal{O}_{X}$-modules (see e.g. [4], Cor. 2.10, 2.11). One only has to check that for every surjection of $\mathcal{A}$-modules $F \rightarrow G$, where $G$ is coherent, there exists a coherent $\mathcal{A}$-submodule $F_{0} \subset F$ that still surjects onto $G$. We can start with a coherent subsheaf $F^{\prime} \subset F$ surjecting onto $G$ and then replace it with the image of the corresponding morphism of $\mathcal{A}$-modules $\mathcal{A} \circ \circ_{X} F^{\prime} \rightarrow F$.

Examples. 1. If $\mathcal{A}$ is a $D$-algebra on $X$ (see [7]), flat as a left and as a right $\mathcal{O}_{X^{-}}$ module, then the associated sheaf $\delta \mathcal{A}$ on $X \times X$ has a structure of pure kernel algebra, and the modules over $\delta \mathcal{A}$ are exactly modules over $\mathcal{A}$ in the usual sense. Note that - -flatness of $\delta \mathcal{A}$ over $X$ (with respect to either projection) reduces to the usual flatness, since $\delta \mathcal{A}$ is supported on the diagonal.

2. Let $G$ be a discrete group acting on a scheme $X$. Then we have a natural pure kernel algebra structure on

$$
\mathcal{A}_{X}^{G}:=\bigoplus_{g \in G} \mathcal{O}_{\Gamma_{g}},
$$

where $\Gamma_{g} \subset X \times X$ is the graph of the action of $g^{-1} \in G$ on $X$, i.e., $\Gamma_{g}=\{(g x, x) \mid$ $x \in X\}$. The product is induced by the natural isomorphisms $\mathcal{O}_{\Gamma_{g}{ }^{\circ} X} \mathcal{O}_{\Gamma_{g^{\prime}}} \simeq \mathcal{O}_{\Gamma_{g g^{\prime}}}$. For every $F \in \mathrm{Q} \operatorname{coh}(X)$ we have natural isomorphisms $\mathcal{O}_{\Gamma_{g}}{ }^{\circ} X=\left(g^{-1}\right)^{*} F$. It is easy to see that $\mathcal{A}_{X}^{G}$-modules are exactly $G$-equivariant quasi-coherent sheaves on $X$. Later we will generalize this example to actions of finite group schemes and of formal groups (see Corollary 3.3.2).

3. Assume that $X$ and $S$ are affine, $X=\operatorname{Spec} A, S=\operatorname{Spec} R$. Then a pure kernel algebra $\mathcal{A}$ over $X$ corresponds to an associative ring $\Gamma(\mathcal{A})$ equipped with a 
homomorphism $A \rightarrow \Gamma(\mathcal{A})$ such that the image of $R$ is in the centre of $\Gamma(\mathcal{A})$, and such that $\Gamma(\mathcal{A})$ is flat as a left (resp. right) $A$-module. Modules over $\mathcal{A}$ are usual modules over $\Gamma(\mathcal{A})$.

We are going to describe several basic operations with kernel algebras. Let us denote by $\sigma: Y \times X \rightarrow X \times Y$ the permutation isomorphism. Then for $K \in$ $D_{\mathrm{qc}}(X \times Y)$ and $L \in D_{\mathrm{qc}}(Y \times Z)$ we have a natural isomorphism

$$
\sigma^{*}\left(K \circ_{Y} L\right) \simeq \sigma^{*} L \circ_{Y} \sigma^{*} K
$$

on $Z \times X$.

Definition. For a kernel algebra $\mathcal{A}$ over $X$ the opposite kernel algebra $\mathcal{A}^{\text {opp }}$ is $\sigma^{*} \mathcal{A}$, where $\sigma$ is the permutation of factors in $X \times X$. The product structure is induced by the product structure on $\mathcal{A}$ using the isomorphism (2.2.1). Note that the opposite of a pure kernel algebra is pure. The isomorphism (2.2.1) also shows that in the pure case there is an equivalence of the category of left $\mathcal{A}^{\mathrm{opp}}$-modules with the category of right $\mathcal{A}$-modules (and vice versa).

For $K \in D_{\mathrm{qc}}(X \times Y)$ and $K^{\prime} \in D_{\mathrm{qc}}\left(X^{\prime} \times Y^{\prime}\right)$ we denote the external product

$$
K \square K^{\prime}=\sigma_{23}^{*}\left(p_{X Y}^{*} K \otimes p_{X^{\prime} Y^{\prime}}^{*} K^{\prime}\right) \in D_{\mathrm{qc}}\left(X \times X^{\prime} \times Y \times Y^{\prime}\right),
$$

where $\sigma_{23}: X \times X^{\prime} \times Y \times Y^{\prime} \rightarrow X \times Y \times X^{\prime} \times Y^{\prime}$ is the transposition.

Lemma 2.2.4. (i) For $K \in D_{\mathrm{qc}}(X \times Y), L \in D_{\mathrm{qc}}(Y \times Z), K^{\prime} \in D_{\mathrm{qc}}\left(X^{\prime} \times Y^{\prime}\right)$ and $L^{\prime} \in D_{\mathrm{qc}}\left(Y^{\prime} \times Z^{\prime}\right)$ one has

$$
\left(K \square K^{\prime}\right){ }^{\circ}{ }_{\times Y^{\prime}}\left(L \square L^{\prime}\right) \simeq\left(K \circ_{Y} L\right) \square\left(K^{\prime} \circ^{\prime} Y^{\prime} L^{\prime}\right)
$$

provided $Y$ and $Y^{\prime}$ are flat over $S$.

(ii) For $K$ and $K^{\prime}$ as above and for $F \in D_{\mathrm{qc}}\left(Y \times Y^{\prime}\right)$ one has a natural isomorphism on $X \times X^{\prime}$,

$$
\left(K \square K^{\prime}\right){ }^{\circ} Y \times Y^{\prime} F \simeq\left(K \circ_{Y} F\right){ }^{\circ} Y^{\prime}\left(\sigma^{*} K^{\prime}\right),
$$

provided either $X^{\prime}$ or $Y$ is flat over $S$.

(iii) Assume that $X$ and $X^{\prime}$ are flat over $S$. If $K$ (resp. $K^{\prime}$ ) is a quasi-coherent sheaf on $X \times Y$ (resp. $X^{\prime} \times Y^{\prime}$ ), o-flat over $Y$ (resp. $Y^{\prime}$ ) then $K \square K^{\prime}$ is o-flat over $Y \times Y^{\prime}$.

Proof. Parts (i) and (ii) are easy applications of the projection and base change formulae. Part (iii) follows from (ii) and from Lemma 2.1.3 (iii).

Definition. If $\mathcal{A}$ is a kernel algebra over $X$ and $\mathcal{B}$ is a kernel algebra over $Y$ then their external product $\mathcal{A} \square \mathscr{B}$ has a natural structure of the kernel algebra over $X \times Y$ induced by an isomorphism of Lemma 2.2.4 (i). If $\mathcal{A}$ and $\mathcal{B}$ are pure then so is $\mathcal{A} \square \mathscr{B}$ (the required o-flatness follows from Lemma 2.2.4 (iii)). 
Lemma 2.2.5. Let $\mathcal{A}$ (resp. $\mathscr{B})$ be a pure kernel algebra over $X$ (resp. $Y$ ). Let $F$ be a quasi-coherent sheaf on $X \times Y$. Then an $\mathcal{A} \square \mathscr{B}^{\text {opp }}$-module structure on $F$ is determined by a pair of morphisms $\alpha: \mathcal{A}^{\circ} \circ_{X} F \rightarrow F, \beta: F \circ_{Y} \mathscr{B} \rightarrow F$ that satisfy the left and right module axioms and that commute with each other, i.e., the diagram

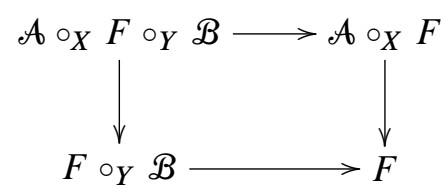

is commutative.

Proof. This follows easily from the natural isomorphism

$$
\left(\mathcal{A} \square \mathscr{B}^{\text {opp }}\right){ }^{X} \times \times Y=A \circ_{X} F \circ_{Y} \mathscr{B}
$$

provided by Lemma 2.2.4(ii).

For example, taking $\mathscr{B}=\Delta_{*} \mathcal{O}_{Y}$ we see that an $\mathcal{A} \square \Delta_{*} \mathcal{O}_{Y}$-module structure on a quasi-coherent sheaf $F$ on $X \times Y$ is given by a morphism $\mathcal{A}{ }^{\circ} X F \rightarrow F$ on $X \times Y$ satisfying the module axioms.

Definition. Let $f: X \rightarrow Y$ be a morphism of flat $S$-schemes. If $\mathcal{A}$ is a kernel algebra over $X$ then $(f \times f)_{*} \mathcal{A}$ has a natural structure of a kernel algebra over $Y$ induced by the morphism

$$
(f \times f)_{*} \mathcal{A} \circ_{Y}(f \times f)_{*} \mathcal{A} \rightarrow(f \times f)_{*}(\mathcal{A} \circ X \mathcal{A})
$$

(see (2.1.3)). If in addition $f$ is flat and affine, $Y$ is flat over $S$, and $\mathcal{A}$ is pure, then $(f \times f)_{*} \mathcal{A}$ is also pure. Indeed, the fact that $(f \times f)_{*} \mathcal{A}$ is o-flat over $Y$ follows from Lemma 2.1.3 (i). We call the kernel algebra $(f \times f)_{*} \mathcal{A}$ the push-forward of $\mathcal{A}$ under $f$.

Lemma 2.2.6. Let $f: X \rightarrow Y$ be a flat affine morphism, where $Y$ is flat over $S$. Then for a pure kernel algebra $\mathcal{A}$ over $X$ the functor $F \mapsto f_{*} F$ induces an equivalence of the category of A-modules with the category of $(f \times f)_{*} \mathcal{A}$-modules. If in addition $f$ is finite and $\mathcal{A}$ is finite then so is $(f \times f)_{*} \mathcal{A}$ and we have an equivalence $\mathcal{A}-\bmod ^{\mathrm{c}} \simeq(f \times f)_{*} \mathcal{A}-\bmod ^{\mathrm{c}}$.

Proof. Note that the assertion is clear in the case when $Y$ and $S$ are affine. In the general case, for an $\mathcal{A}$-module $F$ the natural $(f \times f)_{*} \mathcal{A}$-module structure on $f_{*} F$ is given by the following composition

$$
(f \times f)_{*} \mathcal{A} \circ Y f_{*} F \stackrel{\sim}{\longrightarrow} f_{*}\left(\mathcal{A} \circ X f^{*} f_{*} F\right) \rightarrow f_{*}(\mathcal{A} \circ X F) \rightarrow f_{*} F,
$$


where the first isomorphism is derived from (2.1.2), and the second arrow is induced by the adjunction map $f^{*} f_{*} F \rightarrow F$. Conversely, assume we are given an $(f \times f)_{*} \mathcal{A}$ module structure on a quasi-coherent sheaf $G$ over $Y$. Then it induces an $f_{*} \mathcal{O}_{X^{-}}$ module structure on $G$ so that $G \simeq f_{*} F$. Furthermore, it is easy to see that the structure morphism from $(f \times f)_{*} \mathcal{A}^{\circ} \circ_{Y} f_{*} F \simeq f_{*}\left(\mathcal{A}^{\circ}{ }_{X} f^{*} f_{*} F\right)$ to $f_{*} F$ commutes with the $f_{*} \mathcal{O}_{X}$-module structures on both sides, so it is induced by a morphism $\mathcal{A}{ }^{\circ} X f^{*} f_{*} F \rightarrow F$. We claim that this morphism factors through the canonical morphism $\alpha: \mathcal{A}^{\circ}{ }_{X} f^{*} f_{*} F \rightarrow \mathcal{A}^{\circ}{ }_{X} F$. Indeed, since $\alpha$ is surjective it is enough to check this locally. But in the affine case we know this to be true. The associativity of the obtained action $\mathcal{A} \circ_{X} F \rightarrow F$ can also be checked locally.

In the case when $f$ is finite the functor $f_{*}$ preserves coherence, which implies the second assertion.

Remark. Let $\mathcal{A}$ be a pure kernel algebra on $X$, and let $g: Y \rightarrow Y^{\prime}$ be an affine morphism of flat $S$-schemes. Then for an $\mathcal{A} \square \Delta_{*} \mathcal{O}_{Y}$-module $F$ on $X \times Y$ we can introduce a natural $\mathcal{A} \square \Delta_{*} \mathcal{O}_{Y^{\prime}}$-module structure on $\left(\operatorname{id}_{X} \times g\right)_{*} F$. Namely, it is given by the map

$$
\mathcal{A} \circ_{X}\left(\operatorname{id}_{X} \times g\right)_{*} F \simeq\left(\operatorname{id}_{X} \times g\right)_{*}(\mathcal{A} \circ X F) \rightarrow\left(\operatorname{id}_{X} \times g\right)_{*} F,
$$

where the first isomorphism follows from Lemma 2.1.2 (ii). If in addition $g$ is flat then by Lemma 2.2.6, we can view $\left(\operatorname{id}_{X} \times g\right)_{*} F$ as a module over the pure kernel algebra $\mathcal{A} \square \Delta_{*} g_{*} \mathcal{O}_{Y}$, and the above $\mathcal{A} \square \Delta_{*} \mathcal{O}_{Y^{\prime}}$-module structure is induced by the homomoprhism of kernel algebras $\mathcal{A} \square \Delta_{*} \mathcal{O}_{Y^{\prime}} \rightarrow \mathcal{A} \square \Delta_{*} g_{*} \mathcal{O}_{Y}$.

2.3. Čech resolutions. We are going to introduce a notion of compatibility of a kernel algebra $\mathcal{A}$ with an open covering so that the Čech resolution of an $\mathcal{A}$-module with respect to this covering would inherit the $\mathcal{A}$-module structure. Here is a general framework for this construction.

Definition. Let $(\ell, *)$ be a monoidal category with a unit object $I$, and let $(A, \mu: A *$

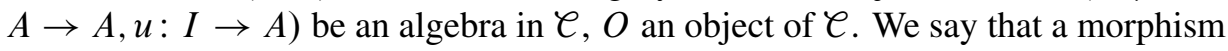

$$
\sigma: A * O \rightarrow O * A
$$

is compatible with the algebra structure on $A$ if the diagram

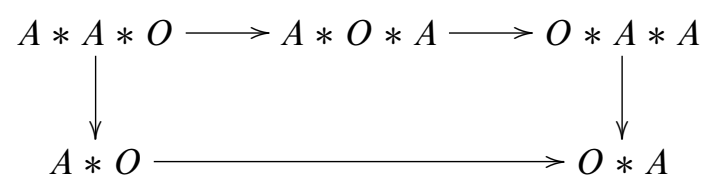

is commutative, where the horizontal (resp. vertical) arrows are induced by $\sigma$ (resp. $\mu$ ), and in addition $\sigma\left(u * \mathrm{id}_{O}\right)=\operatorname{id}_{O} * u$ (equality of morphisms from $O$ to $O * A$ ). 
The next lemma gives a pattern for constructing complexes similar to Čech resolutions.

Lemma 2.3.1. Let $(\mathcal{C}, *), A,(O, \sigma)$ be as in the above definition. Assume in addition that the category $C$ is additive (where the monoidal structure is given by an additive functor $)$, and $O$ is equipped with a morphism $\iota: I \rightarrow O$ such that $\sigma\left(\mathrm{id}_{A} * \iota\right)=\iota * \mathrm{id}_{A}$ (equality of morphisms from $A$ to $O * A$ ). Then there is a natural structure of a $d g$ algebra (with a unit) over $(\mathcal{C}, *)$ on

$$
T^{\bullet}(O) * A:=A \oplus O * A \oplus O * O * A \oplus \cdots,
$$

where multiplication is induced by the product on $A$ and $\sigma$, the differential $d$ is characterized by

$$
d(A)=0,\left.\quad d\right|_{O}=\left(\operatorname{id}_{O} * \iota-\iota * \operatorname{id}_{O}\right) * \operatorname{id}_{A}: O * A \rightarrow O * O * A .
$$

The morphism $\iota * \mathrm{id}_{A}: A \rightarrow O *$ A defines a map of complexes of $A$-bimodules

$$
A \rightarrow T^{>0}(O) * A[1] .
$$

Similarly, for every left A-module $M$ there is a natural structure of a left (unital) dg-module over $T^{\bullet}(O) * A$ on $T^{\bullet}(O) * M$ and a map of complexes of $A$-modules $M \rightarrow T^{>0}(O) * M[1]$.

The proof is left to the reader.

We will apply this lemma to the monoidal structure given by the convolution. The algebra $A$ will be a kernel algebra and the object $O$ will be the sheaf $\Delta(\mathfrak{U})$ concentrated on the diagonal associated with an open covering $\mathcal{U}$ (see (2.3.1) below). The obtained complex of $A$-modules $T^{>0}(O) * M[1]$ will be the Čech resolution.

By an open covering $\mathfrak{U}=\left(U_{i}\right)$ of $X$ we always mean an open covering with respect to the flat (fppf) topology. For a quasi-coherent sheaf $F$ on $X$ and an open covering $\left(U_{i}\right)_{i \in I}$ of $X$ such that the maps $j_{i}: U_{i} \rightarrow X$ are affine, let us denote by $C_{\mathfrak{U}}(F)$ the corresponding Čech resolution of $F$ (where indices are allowed to coincide). Namely,

$$
C_{\mathfrak{U}}^{p}(F)=\bigoplus_{J \subset I p+1} j_{J, *} j_{J}^{*} F,
$$

where for $J=\left(i_{0}, \ldots, i_{p}\right)$ we denote by $j_{J}: U_{i_{0}} \times_{X} \cdots \times_{X} U_{i_{p}} \hookrightarrow X$ the natural embedding. Note that in this case all the fibered products of $j_{i}$ 's are still affine maps, so the corresponding push-forward functors are exact. For example, we can take Čech resolutions corresponding to affine open coverings (the corresponding morphisms $j_{i}: U_{i} \rightarrow X$ will be automatically affine since $X$ is semi-separated).

These Čech resolution can be viewed in the context of Lemma 2.3.1 as follows. Consider the following sheaf on $X \times X$ :

$$
\Delta(\mathfrak{U}):=\Delta_{*} C_{\mathfrak{U}}^{0}\left(\mathcal{O}_{X}\right)=\bigoplus_{i} \Delta_{*} j_{i *} \mathcal{O}_{U_{i}}
$$


It is equipped with the natural map $\iota: \Delta_{*} \mathcal{O}_{X} \rightarrow \Delta(\mathfrak{U})$, hence we have the corresponding dg-algebra $T^{\bullet}(\Delta(\mathfrak{U}))$ with respect to the circle product. Set $\mathcal{O}^{\bullet}(X, \mathfrak{U}):=$ $T^{>0}(\Delta(\mathcal{U}))[1]$. It is easy to see that

$$
\mathcal{O}^{\bullet}(X, \mathfrak{U})=\Delta_{*} \mathcal{\iota}_{\mathfrak{U}}^{\bullet}\left(\mathcal{O}_{X}\right)
$$

Furthermore, for $F \in \mathrm{Q} \operatorname{coh}(X \times Y)$ (resp. $G \in \mathrm{Q} \operatorname{coh}(Y \times X))$ we have natural isomorphisms of resolutions of $F$ (resp. $G$ )

$$
\begin{aligned}
\mathcal{O}^{\bullet}(X, \mathcal{U}){ }^{\circ} X & \simeq C_{\left(U_{i} \times Y\right)}^{\bullet}(F), \\
G{ }^{\circ} X \mathcal{O}^{\bullet}(X, \mathcal{U}) & \simeq C_{\left(Y \times U_{i}\right)}^{\bullet}(G) .
\end{aligned}
$$

Using Lemma 2.3.1 we arrive to the following definition which specifies when Čech resolutions for modules over a kernel algebra still carry an action of this kernel algebra.

Definition. We say that an open covering $\left(U_{i}\right)$ of $X$ (with affine maps $j_{i}: U_{i} \rightarrow X$ ) is compatible with a kernel algebra $\mathcal{A}$ over $X$ if there is an isomorphism in $D(X \times X)$

$$
\sigma: \mathcal{A} \circ_{X} \Delta(\mathfrak{U}) \stackrel{\sim}{\longrightarrow} \Delta(\mathcal{U}) \circ_{X} \mathcal{A}
$$

compatible with the kernel algebra structure on $\mathcal{A}$ and with the canonical homomorphism $\Delta_{*} \mathcal{O}_{X} \rightarrow \Delta(\mathfrak{U})$. We say that a pure kernel algebra $\mathcal{A}$ over $X$ is of affine type if there exists an open affine covering $\left(U_{i}\right)$ of $X$ with respect to the flat topology, compatible with $\mathcal{A}$.

Examples. 1. Let $\pi: X \rightarrow S$ be the projection. Then for any open covering $\left(S_{i}\right)$ of $S$ such that the maps $S_{i} \rightarrow S$ are affine, the induced open covering $\left(U_{i}=S_{i} \times{ }_{S} X\right)$ of $X$ is compatible with any kernel algebra over $X$. Indeed, this follows from the natural isomorphisms of $X \times_{S} X$-schemes for each $i$ :

$$
U_{i} \times{ }_{S} X \simeq S_{i} \times{ }_{S}\left(X \times_{S} X\right) \simeq\left(X \times_{S} X\right) \times_{S} S_{i} \simeq X \times{ }_{S} U_{i}
$$

2. Let $\mathcal{A}$ be a pure kernel algebra over $X$. If $\left(U_{i}\right)$ is an open affine covering of $X$ with respect to the Zariski topology and the support of $\left.\mathcal{A}\right|_{U_{i} \times X}\left(\operatorname{resp} .\left.\mathcal{A}\right|_{X \times U_{i}}\right)$ is contained in $U_{i} \times U_{i}$ for every $i$, then $\left(U_{i}\right)$ is compatible with $\mathcal{A}$. For example, if $\mathcal{A}$ is supported on the diagonal in $X \times X$ (i.e., comes from a $D$-algebra) then $\mathcal{A}$ is of affine type.

3. Assume that $\mathcal{A}$ is associated with an action of a discrete group $G$ on $X$ (see Example 2 in Section 2.2). Take an open affine covering $\left(U_{i}\right)_{i \in I}$ of $X$ such that $G$ permutes the open subsets $U_{i}$. In other words, we assume that there is an action of $G$ on the set of indices $I$ and isomorphisms $a_{g}: U_{i} \stackrel{\sim}{\longrightarrow} U_{g(i)}$ defining the action of $G$ on $\bigsqcup_{i} U_{i}$ such that for each $g \in G$ the diagram

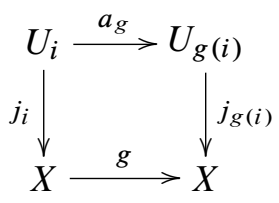


is commutative. Then it is easy to check that the covering $\left(U_{i}\right)$ is compatible with $\mathcal{A}$. For a generalization, see Proposition 3.6.2.

Assume that an open affine covering $\mathfrak{U}=\left(U_{i}\right)$ is compatible with a pure kernel algebra $\mathcal{A}$ over $X$. Then by Lemma 2.3.1, we have a natural resolution of $\mathcal{A}$ by $\mathcal{A} \square \mathcal{A}^{\mathrm{opp}}$-modules on $X \times X$,

$$
\mathcal{A}^{\bullet}(\mathfrak{U}):=\mathcal{O}^{\bullet}(X, \mathfrak{U}) \circ_{X} \mathcal{A} \simeq \mathcal{A} \circ_{X} \mathcal{O}^{\bullet}(X, \mathfrak{U}),
$$

which can be identified with the Čech resolution of $\mathcal{A}$ with respect to either covering, $\left(U_{i} \times X\right)$ or $\left(X \times U_{i}\right)$.

We will also need truncated Čech resolutions. First, for every $N>0$ consider the complex $\tau_{\leq N} C_{\left(U_{i}\right)}^{\cdot}\left(\mathcal{O}_{X}\right)$. Clearly it is still a resolution of $\mathcal{O}_{X}$. We are going to show that for sufficiently large $N$ this truncation will be a sufficiently good replacement for the Čech complex (see Lemma 2.3.3 below).

Lemma 2.3.2. Let $\mathfrak{U}=\left(U_{i}\right)$ be an open covering of $X$ such that the morphisms $j_{i}: U_{i} \rightarrow X$ are affine.

(i) For every $N>0$ the kernel of the differential $K^{N}=\operatorname{ker}\left(d^{N}: C_{\mathfrak{u}}^{N}\left(\mathcal{O}_{X}\right) \rightarrow\right.$ $\left.C_{\mathfrak{U}}^{N+1}\left(\mathcal{O}_{X}\right)\right)$ is locally a direct summand of $C_{\mathfrak{u}}^{N-1}\left(\mathcal{O}_{X}\right)$.

(ii) If $F$ is a quasi-coherent sheaf on $X$ then we have

$$
\tau_{\leq N} C_{\mathfrak{U}}(F) \simeq\left(\tau_{\leq N} C_{\mathfrak{u}}\left(\mathcal{O}_{X}\right)\right) \otimes F \simeq \tau_{\leq N} \mathcal{O}^{\bullet}(X, \mathfrak{U}){ }^{\circ} X
$$

Proof. (i) Localizing we can assume that one of the open subsets is $X$ itself. In this case we have a contracting homotopy $h$ for the complex

$$
\mathcal{O}_{X} \rightarrow C_{\mathfrak{U}}^{0}\left(\mathcal{O}_{X}\right) \rightarrow C_{\mathfrak{U}}^{1}\left(\mathcal{O}_{X}\right) \rightarrow \cdots
$$

Restricting $h$ to $K^{N}$ we get a splitting $K^{N} \rightarrow C_{\mathfrak{U}}^{N-1}\left(\mathcal{O}_{X}\right)$ of the surjection $C_{\mathfrak{U}}^{N-1}\left(\mathcal{O}_{X}\right) \rightarrow K^{N}$ induced by $d^{N-1}$. Hence $K^{N}$ is a direct summand of $C_{\mathfrak{U}}^{N-1}\left(\mathcal{O}_{X}\right)$.

(ii) As we have seen in (i), the short exact triples $0 \rightarrow K^{N-1} \rightarrow C^{N-1} \rightarrow K^{N} \rightarrow$ 0 are locally split. Therefore, tensoring them with any quasi-coherent sheaf we still get exact triples.

From our point of view the key feature of the Čech resolution is that it transforms flat sheaves into complexes of o-flat sheaves. Let us show that the same is true for sufficiently big truncated Čech resolutions.

Lemma 2.3.3. Let $V$ be a flat quasi-coherent sheaf on $X$, and let $\mathfrak{U}=\left(U_{i}\right)$ be an open affine covering of $X$ (with respect to the flat topology). Let $n_{0}$ be the integer such that $H^{i}(X, F)=0$ for $i>n_{0}$ for all quasi-coherent sheaves $F$. Then for $N>n_{0}$ all terms of the truncated Čech resolution $\tau_{\leq N} C_{\mathfrak{U}}(V)$ are o-flat over $X$. 
Proof. First, we note that by Lemma 2.1.3(i), (ii), all the terms $C_{\mathfrak{u}}^{n}(V)$ are o-flat over $X$ (because $V$ is flat over $X$ ). Therefore, we only have to check that $K^{N}(V)=$ $\operatorname{ker}\left(C_{\mathfrak{U}}^{N}(V) \rightarrow C_{\mathfrak{u}}^{N+1}(V)\right)$ is o-flat over $X$. Recall that by Lemma 2.3.2, $K^{N}(V)$ is the tensor product of $V$ with a flat sheaf $K^{N}\left(\mathcal{O}_{X}\right)$, so it is flat over $X$. Therefore, for every quasi-coherent sheaf $G$ on $X$ the sequence of sheaves on $X$

$$
0 \rightarrow V \otimes G \rightarrow C_{\mathfrak{u}}^{0}(V) \otimes G \rightarrow \cdots \rightarrow C_{\mathfrak{u}}^{N-1}(V) \otimes G \rightarrow K^{N}(V) \otimes G \rightarrow 0
$$

is exact. Since in this sequence all the sheaves $C_{\mathfrak{U}}^{i}(V) \otimes G$ are $\pi$-acyclic, where $\pi: X \rightarrow S$ is the projection, our assumption $N>n_{0}$ implies that $K^{N}(V) \otimes G$ is also $\pi$-acyclic. Hence,

$$
G \circ_{X} K^{N}(V)=\pi_{*}\left(K^{N}(V) \otimes G\right)
$$

is concentrated in degree 0 , i.e., $K^{N}(V)$ is o-flat over $X$.

2.4. Convolution for modules over kernel algebras. Let $(X, \mathcal{A}),(Y, \mathcal{B}),(Z, \mathcal{C})$ be three (flat) $S$-schemes equipped with pure kernel algebras. We would like to find an analog of the convolution operation considered in Section 2.1 for modules. Namely, for $F \in D\left(\mathcal{A} \square \mathscr{B}^{\text {opp }}\right.$-mod) and $G \in D\left(\mathscr{B} \square \mathcal{C}^{\text {opp }}\right.$-mod) we would like to define $F \circ_{\mathscr{B}} Y \in D\left(\mathcal{A} \square \mathcal{C}^{\text {opp }}\right.$-mod) that would globalize the operation of tensor product of bimodules. Below we will show how to do this under a technical assumption on $\mathscr{B}$ (see Proposition 2.4.6). We start by defining an underived version and then will use appropriate resolutions.

Let $F$ be a (left) $\mathcal{A} \square \mathscr{B}^{\text {opp }}$-module, and let $G$ be a $\mathscr{B} \square \mathcal{C}^{\text {opp }}$-module. Assume that either $F$ or $G$ is o-flat over $Y$. Then we define

$$
F \bar{\circ}_{\mathcal{B}} G=\operatorname{coker}\left(F \circ_{Y} \mathfrak{B} \circ_{Y} G \stackrel{\alpha}{\rightarrow} F \circ_{Y} G\right),
$$

where $\alpha$ is the difference of two natural maps, one induced by the left action $\mathscr{B}^{\circ}{ }_{Y} G \rightarrow$ $G$ and the other by the right action $F \circ_{Y} \mathscr{B} \rightarrow F$. Note that $F \circ_{Y} G$ and $F \circ_{Y}{ }^{\circ} \circ_{Y} G$ are sheaves because of our o-flatness assumption. The left action of $\mathcal{A}$ on $F$ and the right action of $\mathcal{C}$ on $G$ induce the structure of the $\mathcal{A} \square \mathcal{C}^{\text {opp }}$-module on $F \bar{\circ}_{\mathcal{B}} G$ (see Lemma 2.2.5).

Note that if $\mathcal{A}^{\prime} \rightarrow \mathcal{A}$ (resp. $\mathcal{C}^{\prime} \rightarrow \mathcal{C}$ ) is a homomorphism of pure kernel algebras on $X$ (resp. $Z$ ) then the operation $F \circ_{Y} G$ is compatible with the natural restriction of scalars from $\mathcal{A}$ to $\mathcal{A}^{\prime}$ (resp. from $\mathcal{C}^{\prime}$ to $\mathcal{C}^{\prime}$ ). Also, in the case $\mathcal{B}=\Delta_{*} \mathcal{O}_{Y}$ we get the usual circle operation over $Y$ (since in this case $\alpha=0$ ).

Lemma 2.4.1. Let $(X, \mathcal{A}),(Y, \mathcal{B}),(Z, \mathcal{C}),(T, D)$ be schemes equipped with pure kernel algebras, and let $F \in \mathcal{A} \square \mathscr{B}^{\mathrm{opp}}$-mod, $G \in \mathscr{B} \square \mathcal{C}^{\mathrm{opp}}$-mod and $H \in \mathcal{C} \square$ $D^{\text {opp }}$-mod.

(i) One has an isomorphism of $\mathcal{A} \square \mathscr{B}^{\text {opp }}$-modules $F \bar{o}_{\mathcal{B}} \mathfrak{B} \simeq F$. 
(ii) Assume that $F$ is o-flat over $Y$ and $H$ is o-flat over $Z$. Then one has a functorial isomorphism of $\mathcal{A} \square D^{\mathrm{opp}}$-modules

$$
\left(F \bar{\circ}_{\mathcal{B}} G\right) \bar{\iota}_{\mathcal{C}} H \simeq F \bar{\circ}_{\mathscr{B}}\left(G \bar{\circ}_{\mathcal{C}} H\right) .
$$

Proof. (i) This follows easily from Lemma 2.2.2.

(ii) We have

$$
\begin{aligned}
& \left(F \bar{\circ}_{\mathcal{B}} G\right) \bar{\circ}_{\mathcal{C}} H \\
& \quad \simeq \operatorname{coker}\left(\left(F \bar{\circ}_{\mathcal{B}} G\right) \circ_{Z} \mathcal{C} \circ_{Z} H \rightarrow\left(F \bar{\circ}_{\mathcal{B}} G\right) \circ_{Z} H\right) \\
& \quad \simeq \operatorname{coker}\left(F \circ_{Y} G \circ_{Z} \mathcal{C} \circ_{Z} H \oplus F \circ_{Y} \mathscr{B} \circ_{Y} G \circ_{Z} H \stackrel{\delta}{\rightarrow} F \circ_{Y} G \circ_{Y} H\right),
\end{aligned}
$$

where $\delta$ is induced by the appropriate operations between the consecutive factors in the o-products. Similarly, we get an isomorphism $F \bar{\sigma}_{\mathcal{B}}\left(G \bar{o}_{\mathcal{C}} H\right) \simeq \operatorname{coker}(\delta)$.

Definition. Let $F$ be a left $\mathscr{A} \square \mathscr{B}^{\text {opp }}$-module on $X \times Y$, where $\mathcal{A}$ (resp. $\mathscr{B}$ ) is a pure kernel algebra over $X$ (resp. $Y$ ).

(i) We say that $F$ is $\circ$-flat over $\mathscr{B}$ (or simply $\circ_{\mathcal{B}}$-flat) if it is o-flat over $Y$ and the functor

$$
\mathscr{B} \text {-mod } \rightarrow \text { A-mod: } G \mapsto F \bar{\circ}_{\mathcal{B}} G
$$

is exact.

(ii) $F$ is o-free over $\mathscr{B}$ if $F \simeq F_{0}{ }^{\circ} Y \mathcal{B}$, where $F_{0}$ is a $\mathcal{A} \square\left(\Delta_{*} \mathcal{O}_{Y}\right)$-module, o-flat over $Y$.

(iii) A complex $F^{\bullet}$ of left $\mathscr{A} \square \mathscr{B}^{\text {opp }}$-modules on $X \times Y$ is called $q$-o-flat over $\mathscr{B}$ (or $q$ - ${ }_{\mathcal{B}}$-flat) if it has ${ }^{\circ} Y$-flat terms and for any exact complex $G^{\bullet}$ of $\mathscr{B}$-modules on $Y$ the complex $F^{\bullet} \bar{o}_{\mathcal{B}} G^{\bullet}$ is exact (where the definition of $\bar{o}_{\mathcal{B}}$ is extended to complexes in the standard way).

For example, if $\mathscr{B}=\Delta_{*} \mathcal{O}_{Y}$ then $F$ is automatically o-free over $\mathscr{B}$ (and $\circ_{\mathcal{B}}$-flat) provided it is ${ }^{\circ} Y$-flat.

Lemma 2.4.2. (i) Assume that $F \in \mathcal{A} \square \mathscr{B}^{\mathrm{opp}}$-mod is $\circ$-flat over $\mathscr{B}$. Then for every scheme $Z$ equipped with a pure kernel algebra $\mathcal{C}$ the functor

$$
\mathscr{B} \square \mathcal{C}^{\mathrm{opp}} \text {-mod } \rightarrow \mathcal{A} \square \mathcal{C}^{\mathrm{opp}} \text {-mod: } G \mapsto F \overline{\mathrm{o}}_{\mathcal{B}} G
$$

is exact. Similarly, if $F$ is a $q-\circ_{\mathcal{B}}$-flat complex of $\mathcal{A} \square \mathscr{B}^{\mathrm{opp}}$-modules then the natural extension of the above functor to complexes sends exact complexes to exact complexes.

(ii) Assume that $F \in \mathcal{A} \square \mathscr{B}^{\text {opp }}$-mod is o-free over $\mathfrak{B}$. Then $F$ is o-flat over $\mathscr{B}$.

Proof. (i) Composing with the natural forgetful functor we can assume that $\ell=$ $\Delta_{*} \mathcal{O}_{Z}$. Also, we can assume that $Z$ is affine. Then it is enough to check the exactness of the functor $G \mapsto p_{1 *}\left(F_{\bar{o}_{\mathcal{B}}} G\right)$, where $p_{1}: X \times Z \rightarrow X$ is the projection. Now the 
assertion follows from the isomorphism $p_{1 *}\left(F \bar{\circ}_{\mathscr{B}} G\right) \simeq F \bar{\circ}_{\mathcal{B}}\left(p_{1 *} G\right)$, which one can easily derive from Lemma 2.1.2 (ii) (recall that $p_{1 *} G$ has a natural $\mathcal{B} \otimes \Delta_{*} \mathcal{O}_{Z^{-}}$ module structure, see Remark after Lemma 2.2.6 on p. 183). The same argument works for the second statement.

(ii) Assume that $F=F_{0}{ }^{\circ} Y \mathcal{B}$, where $F_{0}$ is a $\mathcal{A} \square\left(\Delta_{*} \mathcal{O}_{Y}\right)$-module on $X \times Y$, o-flat over $Y$. Note that in this case $F$ is also ${ }^{\circ} Y$-flat by Lemma 2.1.3 (iv). It is enough to construct a functorial isomorphism of $\mathcal{A}$-modules

$$
\left(F_{0} \circ_{Y} \mathcal{B}\right) \bar{\circ}_{\mathcal{B}} G \simeq F_{0} \circ_{Y} G
$$

for $G \in \mathscr{B}$-mod. Using the definition and the ${ }^{\circ} Y$-flatness of $F_{0}$ we get

$$
\begin{aligned}
\left(F_{0} \circ_{Y} \mathcal{B}\right) \bar{\circ}_{\mathcal{B}} G & \simeq \operatorname{coker}\left(F_{0} \circ_{Y} \mathcal{B} \circ_{Y} \mathcal{B} \circ_{Y} G \rightarrow F_{0}{ }^{\circ} Y \mathcal{B} \circ_{Y} G\right) \\
& \simeq F_{0}{ }^{\circ} Y \operatorname{coker}\left(\mathcal{B} \circ_{Y} \mathcal{B} \circ_{Y} G \rightarrow \mathcal{B} \circ_{Y} G\right) .
\end{aligned}
$$

By Lemma 2.2.2, this is isomorphic to $F_{0}{ }^{\circ} Y G$.

The following lemma is an analogue of the well known properties of $q$-flatness in the case of the usual tensor product of sheaves (cf. [35], 2.5.4).

Lemma 2.4.3. Let $X$ and $Y$ be schemes equipped with pure kernel algebras $\mathcal{A}$ and $\mathcal{B}$.

(i) Let $\left(F_{i}^{\bullet}\right)$ be an inductive system of complexes of left $\mathcal{A} \square \mathcal{B}^{\mathrm{opp}}$-modules on $X \times Y$ (connected by chain maps). Assume that $F_{i}^{\bullet}$ is $q-\circ \mathscr{B}$-flat for every $i$. Then the same is true for the complex $\lim _{i} F_{i}^{\bullet}$.

(ii) If $F^{\bullet}$ is a bounded above complex of $\mathcal{A} \square \mathcal{B}^{\text {opp }}$-modules with $\circ_{\mathcal{B}}$-flat terms then $F^{\bullet}$ is $q$-० $\mathbb{B}$-flat.

Proof. (i) First we note that the terms of $\lim F_{i}^{\bullet}$ are still ${ }^{\circ} Y$-flat by Lemma 2.1.5 (ii). Furthermore, using the same lemma, one can easily see that for every complex $G^{\bullet}$ of $\mathcal{B}$-modules the natural map

$$
\lim \left(F_{i}^{\bullet} \bar{\circ}_{\mathscr{B}} G^{\bullet}\right) \rightarrow\left(\lim F_{i}^{\bullet}\right) \bar{\circ}_{\mathcal{B}} G^{\bullet}
$$

is an isomorphism. This immediately implies the result.

(ii) Such a complex is the inductive limit of bounded complexes with $\circ_{\mathscr{B}}$-flat terms for which the statement is clear (cf. [35], 2.5.4).

Since we will need resolutions with terms that are o-flat over a given pure kernel algebra, we give the following technical definition.

Definition. (i) Let $\mathcal{A}$ be a pure kernel algebra over $X$, and let $F$ be a $\mathcal{A} \square \Delta_{*} \mathcal{O}_{Y^{-}}$ module on $X \times Y$. We say that $F$ is o-flattening over $\mathcal{A}$ if it is ${ }^{\circ} Y$-flat, and for every flat quasi-coherent sheaf $V$ over $Y$ the $\mathcal{A}$-module $F{ }^{\circ} Y V$ is o-flat over $\mathcal{A}$. 
(ii) A kernel algebra $\mathcal{A}$ over $X$ is called left (resp. right) admissible if it is pure and there exist a quasi-isomorphism (given by a chain map) $\mathcal{A} \rightarrow \mathcal{M}^{\bullet}$, where $\mathcal{M}^{\bullet}$ is a bounded complex of $\mathcal{A} \square \mathcal{A}^{\text {opp }}$-modules on $X \times X$, such that each term $\mathcal{M}^{n}$, viewed as an $\mathcal{A} \square \Delta_{*} \mathcal{O}_{X}$-module (resp. $\Delta_{*} \mathcal{O}_{X} \square \mathcal{A}^{\text {opp }}$-module), is o-flattening over $\mathcal{A}$ (resp. $\mathcal{A}^{\text {opp }}$ ). We say that a kernel algebra is admissible if it is left and right admissible.

The main point of admissibility assumption is that it allows to construct resolutions by modules that are $\circ$-flat over our kernel algebra (see Proposition 2.4.5 below). We will show also that every pure kernel algebra of affine type is admissible, and that the push-forward of an admissible algebra under a flat affine morphism is admissible.

Lemma 2.4.4. Let $\mathcal{A}$ be a pure kernel algebra over a flat $S$-scheme $X$, and let $Y$ and $Z$ be flat $S$-schemes. If $F$ is a $\mathcal{A} \square \Delta_{*} \mathcal{O}_{Y}$-module, o-flattening over $\mathcal{A}$, then for every quasi-coherent sheaf $V$ over $Y \times Z$, flat over $Y$, the $\mathcal{A} \square \Delta_{*} \mathcal{O}_{Z}$-module $F \circ_{Y} V$ is o-flat over $\mathcal{A}$.

Proof. We have to check exactness of the functor

$$
\mathcal{A}-\bmod \rightarrow \mathrm{Q} \operatorname{coh}(Z): G \mapsto G \bar{\circ}_{\mathcal{A}}\left(F \circ_{Y} V\right) .
$$

Without loss of generality we can assume that $Z$ is affine. Then it is enough to check the exactness of the functor

$$
G \mapsto \pi_{*}\left(G \bar{\circ}_{\mathcal{A}}\left(F \circ_{Y} V\right)\right) \simeq G \bar{\circ}_{\mathcal{A}} p_{X *}\left(F \circ_{Y} V\right) \simeq G \bar{\circ}_{\mathcal{A}}\left(F \circ_{Y} p_{Y *}(V)\right),
$$

where $\pi: X \rightarrow S, p_{X}: X \times Z \rightarrow X$ and $p_{Y}: Y \times Z \rightarrow Y$ are the projections. But this follows from the condition that $F$ is o-flattening over $\mathcal{A}$, since $p_{Y *}(V)$ is a flat sheaf on $Y$.

Proposition 2.4.5. Let $(X, \mathcal{A})$ and $(Y, \mathfrak{B})$ be schemes (flat over $S)$ equipped with pure kernel algebras. Assume that $\mathcal{A}$ is left admissible. Then every complex in $D\left(\mathcal{A} \square \mathscr{B}^{\mathrm{opp}}\right)$ is quasi-isomorphic to a $q$-。 ${ }_{\mathcal{A}}$-flat complex of $\mathcal{A} \square \mathscr{B}^{\mathrm{opp}}$-modules.

Proof. First let $F$ be an arbitrary $\mathcal{A} \square \mathscr{B}^{\text {opp }}$-module on $X \times Y$. Then we can find a functorial surjection of quasi-coherent sheaves $P \rightarrow F$, where $P$ is flat over $X \times Y$ (by Lemma 1.1.4; note that $X \times_{S} Y$ is semi-separated). We have the induced surjection of $\mathcal{A} \square \mathscr{B}^{\text {opp }}$-modules $\mathcal{A} \circ_{X} P \circ_{Y} \mathscr{B} \rightarrow F$. Note that $P \circ_{Y} \mathscr{B}$ is still flat over $X$ by Lemma 2.1.3 (v). This implies that for every bounded above complex of $\mathcal{A} \square \mathscr{B}^{\text {opp }}$-modules $F^{\bullet}$ on $X \times Y$ there exists a quasi-isomorphism of the form

$$
c_{1}\left(F^{\bullet}\right)=\mathcal{A} \circ X V^{\bullet} \rightarrow F^{\bullet},
$$

where $V^{\bullet}$ is a bounded above complex of $\left(\Delta_{*} \mathcal{O}_{X}\right) \square \mathscr{B}^{\text {opp }}$-modules, flat over $X$. Next let $\mathcal{A} \rightarrow \mathcal{M}^{\bullet}$ be a quasi-isomorphism of $\mathcal{A}$ with a bounded complex of $\mathcal{A} \square \mathcal{A}^{\text {opp }}$ modules, o-flattening over $\mathcal{A}$ on the left. Consider the natural map of complexes of $\mathcal{A} \square \mathcal{B}^{\text {opp-modules }}$

$$
\alpha: c_{1}\left(F^{\bullet}\right) \rightarrow c_{2}\left(F^{\bullet}\right):=\operatorname{tot}\left[\mathcal{M}^{\bullet} \bar{o}_{\mathcal{A}} c_{1}\left(F^{\bullet}\right)\right]
$$


(where tot denotes passing to the total complex of a bicomplex), induced by the map $\mathcal{A} \rightarrow \mathcal{M}^{\bullet}$. By Lemma 2.4.1, we have isomorphisms

$$
\mathcal{M}^{\bullet} \bar{\circ}_{\mathcal{A}}\left(\mathcal{A} \circ_{X} V^{n}\right) \simeq \mathcal{M}^{\bullet} \circ_{X} V^{n}
$$

of complexes of $\mathcal{A} \square \mathscr{B}^{\text {opp }}$-modules. Since for each $n$ the natural map $\mathcal{A}{ }^{\circ} X V^{n} \rightarrow$ $\mathcal{M}^{\bullet} \circ_{X} V^{n}$ is a quasi-isomorphism, it follows that $\alpha$ is a quasi-isomorphism. On the other hand, applying Lemma 2.4.4 we see that the terms of $c_{2}\left(F^{\bullet}\right)$ are o-flat over $\mathcal{A}$. Hence, by Lemma 2.4 .3 (ii), $c_{2}\left(F^{\bullet}\right)$ is $\circ_{\mathcal{A}}$-flat.

Finally, we use the standard trick to extend the above construction to the case of an unbounded complex of $\mathcal{A} \square \mathcal{B}^{\text {opp }}$-modules $F^{\bullet}$. First, we consider for each $n \geq 0$ the truncated complex $\tau_{\leq n} F^{\bullet}$ and consider the quasi-isomorphisms of bounded above complexes

$$
\tau_{\leq n} F^{\bullet} \leftarrow c_{1}\left(\tau_{\leq n} F^{\bullet}\right) \rightarrow c_{2}\left(\tau_{\leq n} F^{\bullet}\right)
$$

By functoriality we have chain maps $c_{i}\left(\tau_{\leq n} F^{\bullet}\right) \rightarrow c_{i}\left(\tau_{\leq n+1} F^{\bullet}\right)$ for $i=1,2$, commuting with the above quasi-isomorphisms. Now setting $c_{i}\left(F^{\bullet}\right)=\lim _{n} c_{i}\left(\tau_{\leq n} F^{\bullet}\right)$ for $i=1,2$ gives us quasi-isomorphisms

$$
F^{\bullet} \leftarrow c_{1}\left(F^{\bullet}\right) \rightarrow c_{2}\left(F^{\bullet}\right)
$$

Furthermore, by Lemma 2.4.3 (i), the complex $c_{2}\left(F^{\bullet}\right)$ is $q-\circ \mathcal{A}_{\text {-flat. }}$

Returning to the situation in the beginning of this subsection, let $F^{\bullet}$ (resp. $G^{\bullet}$ ) be a complex of $\mathscr{A} \square \mathscr{B}^{\mathrm{opp}}$-modules (resp. $\mathscr{B} \square \mathcal{C}^{\mathrm{opp}}$-modules). Then assuming that $\mathcal{B}$ is right admissible, we define $F^{\bullet} \circ_{\mathscr{B}} G^{\bullet}$ as the total complex associated with the bicomplex $\widetilde{F}^{\bullet} \bar{\circ}_{\mathcal{B}} G^{\bullet}$, where $\widetilde{F}^{\bullet}$ is a $q$-。 ${ }_{\mathcal{B}}$-flat complex quasi-isomorphic to $F^{\bullet}$. Alternatively, if $\mathcal{B}$ is left admissible then we can use a $q-\circ_{\mathcal{B}}$-flat resolution of $G^{\bullet}$.

Proposition 2.4.6. Let $(X, \mathcal{A}),(Y, \mathcal{B}),(Z, \mathcal{C})$ be flat $S$-schemes equipped with pure kernel algebras, where $\mathcal{B}$ is left (resp. right) admissible. Then there is a biexact functor

$$
\begin{gathered}
D\left(\mathcal{A} \square \mathcal{B}^{\mathrm{opp}} \text {-mod }\right) \times D\left(\mathscr{B} \square \mathcal{C}^{\mathrm{opp}} \text {-mod }\right) \rightarrow D\left(\mathcal{A} \square \ell^{\mathrm{opp}} \text {-mod }\right): \\
\left(F^{\bullet}, G^{\bullet}\right) \mapsto F^{\bullet} \circ_{\mathcal{B}} G^{\bullet}
\end{gathered}
$$

defined as above using $q-\circ_{\mathcal{B}}$-flat resolution of $G^{\bullet}\left(\right.$ resp. $\left.F^{\bullet}\right)$. Assume now that $\mathcal{B}$ is right admissible and $\mathcal{C}$ is left admissible, and let $(T, \mathcal{D})$ be another flat $S$-scheme equipped with a pure kernel algebra. Then for $F \in D\left(\mathcal{A} \square \mathscr{B}^{\text {opp }}\right.$-mod), $G \in$ $D\left(\mathcal{B} \square \mathcal{C}^{\mathrm{opp}}\right.$-mod) and $H \in D\left(\mathcal{C} \square \mathscr{D}^{\mathrm{opp}}\right.$-mod) we have a functorial isomorphism in $D\left(\mathcal{A} \square \mathscr{D}^{\text {opp }}\right.$-mod):

$$
\left(F \circ_{B} G\right) \circ_{\mathcal{C}} H \simeq F \circ_{\mathcal{B}}\left(G \circ \circ_{\mathcal{C}} H\right) .
$$

Proof. To prove associativity we choose complexes quasiisomorphic to $F$ and $H$ that are $q$-o-flat over $\mathcal{B}$ and $\ell$, respectively. 
The starting point for constructing examples of admissible kernel algebras is the following result.

Lemma 2.4.7. Let $X$ be a flat $S$-scheme. Then a pure kernel algebra of affine type over $X$ is admissible.

Proof. Let $\mathcal{U}=\left(U_{i}\right)$ be an open affine covering compatible with a kernel algebra $\mathcal{A}$. We claim that for sufficiently large $N$ the truncated Čech resolution $\tau_{\leq N} \mathcal{A}^{\bullet}(\mathfrak{U})=$ $\mathcal{A}{ }^{\circ}{ }_{X} \tau_{\leq N} \mathcal{O}_{X}^{\bullet}(\mathcal{U})$ (see (2.3.5)) has terms which are o-flattening over $\mathcal{A}$ on the left. Since $\tau_{\leq N} \mathcal{O}_{X}^{\circ}(\mathcal{U})$ is o-flat over $X$ (recall that it is supported on the diagonal), it follows that $\tau_{\leq N} \mathcal{A}^{\bullet}(\mathfrak{U})$ is also o-flat over $X$ (on either side). Next, for a flat quasi-coherent sheaf $V$ on $X$, we have

$$
\tau_{\leq N} \mathcal{A}^{\bullet}(\mathfrak{U}){ }^{\circ} X \simeq \mathcal{A} \circ_{X} \tau_{\leq N} \mathcal{O}_{X}^{\bullet}(\mathfrak{U}){ }^{\circ} X V \simeq \mathcal{A} \circ_{X} \tau_{\leq N} C_{\mathfrak{U}}^{\bullet}(V) .
$$

By Lemma 2.3.3, the terms of the complex $\tau_{\leq N} C_{\mathfrak{U}}(V)$ are o-flat over $X$. Hence, the terms of $\tau_{\leq N} \mathcal{A}^{\bullet}(\mathfrak{U}){ }^{\circ} V$ are o-free over $\mathcal{A}$, so they are o-flat over $\mathcal{A}$ by Lemma 2.4.2 (ii). A similar argument shows that the terms of $\tau_{\leq N} \mathcal{A}^{\bullet}(\mathfrak{U})$ are $\circ$-flattening over $\mathcal{A}$ on the right.

Our next goal is to show that admissibility is preserved under push-forwards with respect to affine flat morphisms.

Lemma 2.4.8. Let $\mathcal{B}_{0} \rightarrow \mathcal{B}$ be a homomorphism of pure kernel algebras over $Y$, and let $F$ be an $\mathcal{A} \square \mathcal{B}^{\text {opp }}$-module. Assume that $\mathcal{B}$ and $F$ are o-flat over $\mathcal{B}_{0}$ acting on the right. Then for every $G \in \mathcal{B} \square \mathcal{C}^{\mathrm{opp}}$-mod there is a natural isomorphism of $\mathcal{A} \square$ Copp-modules

$$
F \bar{\circ}_{\mathscr{B}} G \simeq \operatorname{coker}\left(F \bar{\circ}_{\mathcal{B}_{0}} \mathscr{B} \bar{\circ}_{\mathcal{B}_{0}} G \rightarrow F \bar{\circ}_{\mathcal{B}_{0}} G\right)
$$

Proof. This follows easily from the definitions.

Lemma 2.4.9. Let $f: Y \rightarrow Y^{\prime}$ be a flat affine morphism, and let $\mathscr{B}_{f}:=\Delta_{*}\left(f_{*} \mathcal{O}_{Y}\right)$ be the corresponding pure kernel algebra over $Y^{\prime}$. Then $\mathscr{B}_{f}$ is admissible, and for $F \in D_{\mathrm{qc}}(X \times Y)$ and $G \in D_{\mathrm{qc}}(Y \times Z)$ one has a natural isomorphism

$$
\left(\operatorname{id}_{X} \times f\right)_{*} F \circ_{B_{f}}\left(f \times \operatorname{id}_{Z}\right)_{*} G \stackrel{\sim}{\longrightarrow} F \circ_{Y} G .
$$

If $F$ is $a{ }^{\circ}$-flat quasi-coherent sheaf on $X \times Y$ then $\left(\operatorname{id}_{X} \times f\right)_{*} F$ is o-flat over $\mathscr{B}_{f}$.

Proof. The pure kernel algebra $\mathscr{B}_{f}$ is of affine type (being supported on the diagonal), so it is admissible. Hence, the right-hand side of (2.4.3) is well defined. We have a natural morphism

$$
\left(\operatorname{id}_{X} \times f\right)_{*} F \circ_{Y^{\prime}}\left(f \times \operatorname{id}_{Z}\right)_{*} G \rightarrow F \circ_{Y} G
$$


(see (2.1.3)). If $F$ is a sheaf, o-flat over $Y$, then $\left(\operatorname{id}_{X} \times f\right)_{*} F$ is o-flat over $Y^{\prime}$ by Lemma 2.1.3 (i). It is easy to see that for such $F$ and for a quasi-coherent sheaf $G$ on $Y \times Z$ the morphism (2.4.4) induces a morphism

$$
\left(\operatorname{id}_{X} \times f\right)_{*} F \bar{\circ}_{\mathcal{B}_{f}}\left(f \times \operatorname{id}_{Z}\right)_{*} G \rightarrow F \circ_{Y} G .
$$

We claim that in fact this is an isomorphism. Indeed, note that the functor of $G$ on the right-hand side (resp. the left-hand side) is exact (resp. right exact). Also, for every quasi-coherent sheaf $G$ on $Y \times Z$ the natural map $\left(f \times \operatorname{id}_{Z}\right)^{*}\left(f \times \operatorname{id}_{Z}\right)_{*} G \rightarrow G$ is surjective. Thus, it is enough to check that (2.4.5) is an isomorphism for $G=$ $\left(f \times \mathrm{id}_{Z}\right)^{*} G^{\prime}$, where $G^{\prime}$ is a sheaf on $Y \times Z^{\prime}$. To this end we use the isomorphism

$$
\begin{aligned}
\mathscr{B}_{f} \circ_{Y^{\prime}} G^{\prime} \simeq\left((f \times f)_{*} \Delta_{*} \mathcal{O}_{Y}\right) \circ_{Y^{\prime}} G^{\prime} & \simeq\left(f \times \operatorname{id}_{Z}\right)_{*}\left(\Delta_{*} \mathcal{O}_{Y} \circ_{Y}\left(f \times \operatorname{id}_{Z}\right)^{*} G^{\prime}\right) \\
& \simeq\left(f \times \operatorname{id}_{Z}\right)_{*}\left(f \times \operatorname{id}_{Z}\right)^{*} G^{\prime}
\end{aligned}
$$

that follows from (2.1.2). Together with Lemma 2.4.1 (i) this leads to

$$
\left(\operatorname{id}_{X} \times f\right)_{*} F \bar{\circ}_{B_{f}}\left(f \times \operatorname{id}_{Z}\right)_{*}\left(f \times \operatorname{id}_{Z}\right)^{*} G^{\prime} \simeq\left(\operatorname{id}_{X} \times f\right)_{*} F \circ_{Y^{\prime}} G,
$$

which is isomorphic to $F{ }^{\circ} Y\left(f \times \mathrm{id}_{Z}\right)^{*} G^{\prime}$ by Lemma 2.1.2(i). Note that the isomorphism (2.4.5) for $Z=S$ implies that $\left(\operatorname{id}_{X} \times f\right)_{*} F$ is o-flat over $\mathscr{B}_{f}$ (since every $\mathcal{B}_{f}$-module on $Y^{\prime}$ is of the form $f_{*} G$, where $G$ is a quasi-coherent sheaf on $Y$ ). From the considered case we can immediately extend the isomorphism (2.4.3) to the case when $F$ is a bounded above complex of ${ }^{\circ} Y$-flat sheaves on $X \times Y$ and $G$ is arbitrary complex in $D_{\mathrm{qc}}(Y \times Z)$. Finally, if $F$ is unbounded we can consider the ${ }^{\circ} Y$-flat resolutions of the truncations $\tau_{\leq n} F$ and pass to the limit using the isomorphism (2.4.1).

Proposition 2.4.10. Let $f: Y \rightarrow Y^{\prime}$ be a flat affine morphism, $B$ a pure kernel algebra over $Y$, and let $\mathscr{B}^{\prime}:=(f \times f)_{*} \mathscr{B}$ be the corresponding pure kernel algebra over $Y^{\prime}$. Let also $\mathcal{A}$ (resp. $\mathcal{C}$ ) be a pure kernel algebra over $X$ (resp. $Z$ ).

(i) If $F$ is an $\mathcal{A} \square \mathcal{B}^{\mathrm{opp}}$-module, o-flat over $Y$, then $\left(\operatorname{id}_{X} \times f\right)_{*} F$ is o-flat over $Y^{\prime}$ and for $G \in \mathcal{B} \square \mathcal{C}^{\mathrm{opp}}$-mod we have a natural isomorphism

$$
\left(\operatorname{id}_{X} \times f\right)_{*} F \bar{\circ}_{\mathcal{B}^{\prime}}\left(f \times \operatorname{id}_{Z}\right)_{*} G \stackrel{\sim}{\longrightarrow} F \bar{\circ}_{\mathscr{B}} G
$$

In particular, if $F$ is o-flat over $\mathcal{B}$, then $\left(\operatorname{id}_{X} \times f\right)_{*} F$ is o-flat over $\mathscr{B}^{\prime}$.

(ii) If $\mathscr{B}$ is left (resp. right) admissible then so is $\mathscr{B}^{\prime}$. In this case for $F \in$ $D\left(\mathcal{A} \square \mathscr{B}^{\mathrm{opp}}\right.$-mod) and $G \in D\left(\mathcal{B} \square \mathcal{C}^{\mathrm{opp}}\right.$-mod) we have a natural isomorphism

$$
\left(\operatorname{id}_{X} \times f\right)_{*} F \circ_{\mathcal{B}^{\prime}}\left(f \times \operatorname{id}_{Z}\right)_{*} G \simeq F \circ_{B} G
$$

Proof. (i) We have already seen that $\left(\operatorname{id}_{X} \times f\right)_{*} F$ is o-flat over $\mathscr{B}_{f}$ (and over $Y^{\prime}$ ) in Lemma 2.4.9. Note also that by the same Lemma, $\mathscr{B}^{\prime}=\left(\operatorname{id}_{X} \times f\right)_{*}\left(f \times \operatorname{id}_{Y}\right)_{*} \mathscr{B}$ is 
o-flat over $\mathscr{B}_{f}$ (since $\left(f \times \operatorname{id}_{Y}\right)_{*} \mathscr{B}$ is o-flat over $Y$ by Lemma 2.1.3 (i)). Therefore, using Lemma 2.4.8 we can represent the left-hand side of (2.4.6) as the cokernel of

$$
\left(\operatorname{id}_{X} \times f\right)_{*} F \circ_{\mathcal{B}_{f}} \mathscr{B}^{\prime} \circ_{\mathcal{B}_{f}}\left(f \times \operatorname{id}_{Z}\right)_{*} G \rightarrow\left(\operatorname{id}_{X} \times f\right)_{*} F \circ_{\mathcal{B}_{f}} \circ\left(f \times \operatorname{id}_{Z}\right)_{*} G .
$$

Here the target is isomorphic to $F \circ_{Y} G$ by Lemma 2.4.9. Also, using the same Lemma, we can rewrite

$$
\begin{aligned}
\left(\operatorname{id}_{X} \times f\right)_{*} F \circ \circ_{\mathcal{B}_{f}}(f \times f)_{*} \mathcal{B} \circ \circ_{\mathcal{B}_{f}}\left(f \times \operatorname{id}_{Z}\right)_{*} G \\
\quad \simeq\left(\operatorname{id}_{X} \times f\right)_{*} F \circ \circ_{\mathcal{B}_{f}}\left(f \times \operatorname{id}_{Y}\right)_{*} \mathscr{B} \circ_{Y} G \simeq F \circ Y \mathcal{B} \circ_{Y} G,
\end{aligned}
$$

so that the above map is identified with the map $F \circ_{Y} \mathscr{B} \circ_{Y} G \rightarrow F \circ_{Y} G$ used to define $F \bar{\circ}_{\mathcal{B}} G$. This gives the isomorphism (2.4.6).

Since the functor $\left(f \times \mathrm{id}_{Z}\right)_{*}: \mathscr{B} \square \mathcal{C}^{\text {opp }}$-mod $\rightarrow \mathscr{B}^{\prime} \square \mathcal{C}^{\text {opp }}$-mod is an equivalence (see Lemma 2.2.6), the second assertion follows.

(ii) Let $\mathscr{B} \rightarrow \mathcal{M}^{\bullet}$ be a quasi-isomorphism with a bounded complex of $\mathscr{B} \square \mathscr{B}^{\text {opp }}$ modules on $Y \times Y$, such that each term $\mathcal{M}^{n}$ is o-flattening over $\mathcal{B}$ on the left. We claim that $\mathscr{B}^{\prime} \rightarrow(f \times f)_{*} \mathcal{M}^{\bullet}$ is a o-flattening resolution for $\mathscr{B}^{\prime}$. Indeed, let us check that $(f \times f)_{*} \mathcal{M}^{n}$ is o-flattening over $\mathscr{B}^{\prime}$ on the left. First, we note that $(f \times f)_{*} \mathcal{M}^{n}$ is o-flat over $Y^{\prime}$ (on the right) by Lemma 2.1.3 (i). Next, given a flat quasi-coherent sheaf $V$ on $Y^{\prime}$ we have to check that $(f \times f)_{*} \mathcal{M}^{n}{ }^{\circ}{ }_{Y^{\prime}} V$ is o-flat over $\mathscr{B}^{\prime}$. Using (2.1.2) we obtain

$$
(f \times f)_{*} \mathcal{M}^{n} \circ_{Y^{\prime}} V \simeq\left(f \times \operatorname{id}_{Y}\right)_{*}\left(\mathcal{M}^{n} \circ_{Y} f^{*} V\right),
$$

which is o-flat over $\mathscr{B}^{\prime}$ by part (i). Finally, the isomorphism (2.4.7) follows from (2.4.6) by taking a $q-\circ \mathbb{B}^{-}$-flat resolution of $G$ (resp. $F$ ).

\subsection{Extending an equivalence of derived categories to modules over kernel alge-} bras. Recall that a pair of adjoint functors $(\Phi, \Psi)$, where $\Phi: \mathcal{C} \rightarrow \ell^{\prime}, \Psi: \ell^{\prime} \rightarrow \mathcal{C}$, can be characterized by two morphisms of functors $\alpha: \Phi \Psi \rightarrow \mathrm{Id}_{\ell^{\prime}}$ and $\beta: \mathrm{Id}_{\ell} \rightarrow$ $\Psi \Phi$, such that the compositions $\Phi \rightarrow \Phi \Psi \Phi \rightarrow \Phi$ and $\Psi \rightarrow \Psi \Phi \Psi \rightarrow \Psi$ are the identity morphisms. Furthermore, $\Phi$ is fully faithful (resp. equivalence) if and only if $\beta$ is an isomorphism (resp. $\alpha$ and $\beta$ are isomorphisms). For functors given by kernels we can consider the following version of this picture.

Definition. Let $X$ and $Y$ be flat $S$-schemes. Assume that we have objects $\mathcal{P} \in$ $D_{\mathrm{qc}}(X \times Y)$ and $\mathcal{Q} \in D_{\mathrm{qc}}(Y \times X)$ equipped with a pair of morphisms

$$
\begin{aligned}
& \alpha: \mathcal{P} \circ_{Y} \mathcal{Q} \rightarrow \Delta_{*} \mathcal{O}_{X}, \\
& \beta: \Delta_{*} \mathcal{O}_{Y} \rightarrow \mathcal{Q} \circ_{X} \mathcal{P}
\end{aligned}
$$

such that the compositions

$$
\begin{aligned}
& \mathcal{P} \stackrel{\text { id } \mathcal{P} \circ \beta}{\longrightarrow} \mathcal{P} \circ_{Y} \mathcal{Q} \circ_{X} \mathcal{P} \stackrel{\alpha \circ \text { id } \mathcal{P}}{\longrightarrow} \mathcal{P}, \\
& \mathcal{Q} \stackrel{\beta \text { id }_{\mathcal{Q}}}{\longrightarrow} \mathcal{Q} \circ_{X} \mathcal{P} \circ_{Y} \mathcal{Q} \stackrel{\operatorname{id}_{\mathcal{Q}} \circ \alpha}{\longrightarrow} \mathcal{Q}
\end{aligned}
$$


are the identity morphisms. In this situation we say that $(\mathcal{P}, \mathcal{Q} ; \alpha, \beta)$ is an adjoint kernel data. Consider the corresponding functors

$$
\begin{aligned}
& \Phi_{\mathcal{P}}: D_{\mathrm{qc}}(Y) \rightarrow D_{\mathrm{qc}}(X): G \mapsto \mathcal{P} \circ_{Y} G, \\
& \Phi_{\mathcal{Q}}: D_{\mathrm{qc}}(X) \rightarrow D_{\mathrm{qc}}(Y): F \mapsto \mathcal{Q} \circ_{X} F .
\end{aligned}
$$

Then Lemma 2.1.1 implies that $\left(\Phi_{\mathcal{P}}, \Phi_{\mathcal{Q}}\right)$ is an adjoint pair of functors.

Example. Assume $Y$ is smooth and proper over $S$ of relative dimension $n$, and let $\mathcal{P}$ be a perfect complex on $X \times Y$. Then by the duality theory we have a natural adjoint kernel data $(\mathcal{P}, \mathcal{Q})$ with

$$
\mathcal{Q}=\sigma_{*}\left(\mathcal{P}^{\vee} \otimes p_{Y}^{*} \omega_{Y}\right)[n]
$$

where $\sigma: Y \times X \rightarrow X \times Y$ is the permutation of factors, $p_{Y}: X \times Y \rightarrow Y$ is the projection.

Now let $\mathcal{A}$ be a kernel algebra over $X$. Set $\mathscr{B}=\mathcal{Q}^{\circ}{ }_{X} \mathcal{A}{ }^{\circ} X \mathcal{P}$. Then we have a natural morphism

$$
\mathscr{B} \circ_{Y} \mathcal{B} \simeq \mathcal{Q} \circ_{X} \mathcal{A} \circ_{X} \mathcal{P} \circ_{Y} \mathcal{Q} \circ_{X} \mathcal{A} \circ_{X} \mathcal{P} \rightarrow \mathcal{Q} \circ_{X}\left(\mathcal{A} \circ_{X} \mathcal{A}\right) \circ_{X} \mathcal{P},
$$

induced by (2.5.1). Therefore, the product $\mu: \mathcal{A}^{\circ} X \mathcal{A} \rightarrow \mathcal{A}$ induces an associative operation $\mathcal{B}{ }^{\circ} Y \mathcal{B} \rightarrow \mathcal{B}$. Similarly, the unit $u: \Delta_{*} \mathcal{O}_{X} \rightarrow \mathcal{A}$ together with the map (2.5.2) induce a unit morphism $\Delta_{*} \mathcal{O}_{Y} \rightarrow \mathscr{B}$. One can check that the unit axioms for this morphism follow from the compatibilities between $\alpha$ and $\beta$ that we assumed. Thus, we get a structure of a kernel algebra on $\mathcal{B}$.

Note also that (2.5.1) induces morphisms

$$
\begin{aligned}
& \mathcal{P} \circ_{Y} \mathcal{B} \simeq \mathcal{P} \circ_{Y} \mathcal{Q} \circ_{X} \mathcal{A} \circ_{X} \mathcal{P} \rightarrow \mathcal{A} \circ_{X} \mathcal{P}, \\
& \mathcal{B} \circ_{Y} \mathcal{Q} \simeq \mathcal{Q} \circ_{X} \mathcal{A} \circ_{X} \mathcal{P} \circ_{Y} \mathcal{Q} \rightarrow \mathcal{Q} \circ_{X} \mathcal{A},
\end{aligned}
$$

which are compatible with the products (resp. units) on $\mathcal{B}$ and $\mathcal{A}$.

Assume in addition that $\mathcal{A}$ and $\mathscr{B}$ are pure kernel algebras, and that each of the complexes $\mathcal{P}$ and $\mathcal{Q}$ is concentrated in single cohomological degree. Then by Lemma 2.1 .3 (ii), $\widetilde{\mathcal{P}}:=\mathcal{A} \circ_{X} \mathcal{P}$ and $\widetilde{\mathcal{Q}}:=\mathcal{Q}^{\circ}{ }_{X} \mathcal{A}$ are also concentrated in single cohomological degree. Using morphisms (2.5.3) we get the commuting left $\mathcal{A}$-action and right $\mathcal{B}$-action on $\widetilde{\mathcal{P}}$ (resp. left $\mathcal{B}$-action and right $\mathcal{A}$-action on $\widetilde{\mathcal{Q}}$ ). Therefore, using Lemma 2.2 .5 we can view $\widetilde{\mathcal{P}}$ (resp. $\widetilde{\mathcal{Q}}$ ) as an object of $D\left(\mathcal{A} \square \mathscr{B}^{\text {opp }}\right.$-mod) (resp. $D\left(\mathscr{B} \square \mathcal{A}^{\mathrm{opp}}\right.$-mod)). Note that the unit in $\mathcal{A}$ induces morphisms

$$
\iota \mathcal{P}: \mathcal{P} \rightarrow \tilde{\mathcal{P}}, \quad \iota_{\mathcal{Q}}: \mathcal{Q} \rightarrow \widetilde{\mathbb{Q}}
$$

in $D_{\mathrm{qc}}(X \times Y)$ and $D_{\mathrm{qc}}(Y \times X)$. 
Theorem 2.5.1. Assume $S$ is semi-separated. Let $X$ and $Y$ be semi-separated schemes, flat over $S$, and let $\mathcal{P} \in D_{\mathrm{qc}}(X \times Y), \mathcal{Q} \in D_{\mathrm{qc}}(Y \times X)$ be objects, each concentrated in single cohomological degree, equipped with a pair of morphisms (2.5.1) and (2.5.2), such that $(\mathcal{P}, \mathcal{Q}, \alpha, \beta)$ is an adjoint kernel data.

(i) Let $\mathcal{A}$ be an admissible kernel algebra over $X$. Assume that the corresponding kernel algebra $\mathbb{B}=\mathcal{Q}^{\circ}{ }_{X} \mathcal{A}_{X}{ }_{X} \mathcal{P}$ over $Y$ is admissible (in particular, pure). Define $\widetilde{\mathcal{P}} \in D\left(\mathcal{A} \square \mathscr{B}^{\mathrm{opp}}\right.$-mod) and $\widetilde{\mathcal{Q}} \in D\left(\mathscr{B} \square \mathcal{A}^{\mathrm{opp}}\right.$-mod) as above, and consider the functors

$$
\begin{aligned}
& \Phi_{\widetilde{\mathcal{P}}}: D(\mathscr{B} \text {-mod }) \rightarrow D(\mathcal{A} \text {-mod }): G \mapsto \widetilde{\mathcal{P}} \circ_{\mathcal{B}} G, \\
& \Phi_{\widetilde{Q}}: D(\mathcal{A} \text {-mod }) \rightarrow D(\mathscr{B} \text {-mod }): F \mapsto \widetilde{\mathcal{Q}} \circ_{\mathcal{A}} F .
\end{aligned}
$$

Then the natural map in $D_{\mathrm{qc}}(Y \times Y)$ given as the composition

$$
\mathscr{B}=\mathcal{Q} \circ_{X} \mathcal{A} \circ_{X} \mathcal{P} \stackrel{\iota_{Q} \iota_{\mathcal{P}}}{\longrightarrow} \widetilde{\mathcal{Q}} \circ_{X} \mathcal{A} \circ_{X} \widetilde{\mathcal{P}} \rightarrow \widetilde{\mathcal{Q}} \circ_{\mathcal{A}} \tilde{\mathcal{P}}
$$

lifts to an isomorphism in $D\left(\mathscr{B} \square \mathscr{B}^{\mathrm{opp}}\right.$-mod). Hence, we have the induced isomorphism of functors

$$
\Phi_{\tilde{Q}} \circ \Phi_{\tilde{\mathcal{P}}} \simeq \mathrm{Id} .
$$

If in addition $\alpha$ and $\beta$ are isomorphisms then $\Phi_{\widetilde{\mathcal{P}}}$ and $\Phi_{\widetilde{Q}}$ are mutually quasi-inverse equivalences.

(ii) Let $\mathcal{A}_{1} \rightarrow \mathcal{A}$ be a homomorphism of kernel algebras over $X$, and consider the induced homomorphism of kernel algebras $\mathscr{B}_{1}=\mathcal{Q}^{\circ}{ }_{X} \mathcal{A}_{1} \circ \mathcal{P} \rightarrow \mathscr{B}=\mathcal{Q}^{\circ}{ }_{X} \mathcal{A}_{X}{ }_{X} \mathcal{P}$. Suppose that $\mathcal{A}_{,} \mathcal{A}_{1}, \mathscr{B}, \mathscr{B}_{1}$ are all admissible. Then the functors $\Phi_{\tilde{Q}}$ (resp. $\left.\Phi_{\tilde{\mathcal{P}}}\right)$ constructed in (i) for the pairs $(\mathcal{A}, \mathscr{B})$ and $\left(\mathcal{A}_{1}, \mathscr{B}_{1}\right)$ commute with the corresponding restriction (resp. induction) functors between the derived categories of modules.

(iii) Assume in addition that $X$ and $Y$ are proper over $S, \mathcal{P}$ and $\mathcal{Q}$ are coherent sheaves of finite Tor-dimension (up to a shift), and $\mathcal{A}$ is a finite admissible kernel algebra over $X$ such that $\mathscr{B}=Q_{\mathcal{Q}} \circ_{X} \mathcal{A} \circ_{X} \mathcal{P}$ is admissible. Then $\mathscr{B}$ is also finite and the functor $\Phi_{\widetilde{Q}}$ restricts to a functor from $D^{b}\left(\mathcal{A}-\bmod ^{c}\right)$ to $D^{b}\left(\mathscr{B}-\bmod ^{c}\right)$. In particular, when $\alpha$ and $\beta$ are isomorphisms these two categories are equivalent.

Proof. (i) Using the properties of the convolution operation for modules we obtain natural isomorphisms

$$
\widetilde{\mathcal{Q}} \circ_{\mathcal{A}} \tilde{\mathcal{P}} \simeq \widetilde{\mathcal{Q}} \circ_{\mathcal{A}}\left(\mathcal{A} \circ_{X} \mathcal{P}\right) \simeq \widetilde{\mathcal{Q}} \circ_{X} \mathcal{P} \simeq \mathcal{Q} \circ_{X} \mathcal{A} \circ_{X} \mathcal{P}=\mathscr{B}
$$

in $D\left(\mathscr{B} \square \Delta_{*} \mathcal{O}_{Y}\right.$-mod) and

$$
\widetilde{\mathcal{Q}} \circ_{\mathcal{A}} \widetilde{\mathcal{P}} \simeq\left(\mathcal{Q} \circ_{X} \mathcal{A}\right) \circ_{\mathcal{A}} \widetilde{\mathcal{P}} \simeq \mathcal{Q} \circ_{X} \widetilde{\mathcal{P}} \simeq \mathcal{Q} \circ_{X} \mathcal{A} \circ_{X} \mathcal{P}=\mathscr{B}
$$

in $D\left(\Delta_{*} \mathcal{O}_{Y} \square \mathcal{B}^{\text {opp }}\right.$-mod). In particular, $\widetilde{\mathcal{Q}} \circ_{\mathcal{A}} \widetilde{\mathcal{P}}$ is concentrated in degree 0 . Also, it is easy to check that both isomorphisms (2.5.6) and (2.5.7) are compatible with the map (2.5.4). Hence, we get the required isomorphism in $\mathscr{B} \square \mathscr{B}^{\mathrm{opp}}$-mod. The isomorphism 
of functors (2.5.5) follows from this by the associativity of the convolution (see Proposition 2.4.6).

If both $\alpha$ and $\beta$ are isomorphisms then we can reverse the roles of $(X, \mathcal{A})$ and $(Y, \mathcal{B})$ (resp. of $\mathcal{P}$ and $\mathcal{Q}$ ). This will lead to an isomorphism of functors $\Phi_{\widetilde{\mathcal{P}}} \circ \Phi_{\widetilde{\mathcal{Q}}} \simeq \mathrm{Id}$ that together with $(2.5 .5)$ proves that $\Phi_{\tilde{\mathcal{P}}}$ and $\Phi_{\widetilde{Q}}$ are mutually quasi-inverse.

(ii) Let $\widetilde{\mathcal{P}}$ and $\widetilde{\mathcal{Q}}$ have the same meaning as in (i), and let $\widetilde{\mathcal{P}}_{1}:=\mathcal{A}_{1} \circ_{X} \mathcal{P}$ and $\widetilde{Q}_{1}:=\mathcal{Q}_{X} \mathcal{A}_{1}$ be similar objects for $\mathcal{A}_{1}$. Then we have an isomorphism $\widetilde{\mathcal{Q}} \simeq \widetilde{\mathcal{Q}}_{1} \circ \mathcal{A}_{1} \mathcal{A}$ in $D\left(\mathscr{B} \square \mathcal{A}^{\text {opp }}\right.$-mod $)$. Therefore, for $F \in D(\mathcal{A}$-mod $)$ we get

$$
\Phi_{\widetilde{\mathcal{Q}}}(F)=\widetilde{\mathcal{Q}} \circ_{\mathcal{A}} F \simeq\left(\widetilde{\mathcal{Q}}_{1} \circ_{\mathcal{A}_{1}} \mathcal{A}\right) \circ_{\mathcal{A}} F \simeq \widetilde{\mathcal{Q}}_{1} \circ_{\mathcal{A}_{1}} F=\Phi_{\widetilde{Q}_{1}}(F),
$$

from which we obtain the compatibility of $\left(\Phi_{\widetilde{Q}_{\tilde{Q}}}, \Phi_{\widetilde{Q}_{1}}\right)$ with the restriction functors $D(\mathcal{A}$-mod $) \rightarrow D\left(\mathcal{A}_{1}\right.$-mod $)$ and $D(\mathscr{B}$-mod $) \rightarrow D\left(\mathscr{B}_{1}\right.$-mod $)$. On the other hand, using the isomorphism $\widetilde{\mathcal{P}} \simeq \mathcal{A}_{\mathcal{A}_{1}} \widetilde{\mathcal{P}}_{1}$, for $G \in D\left(\mathscr{B}_{1}\right.$-mod $)$ we obtain

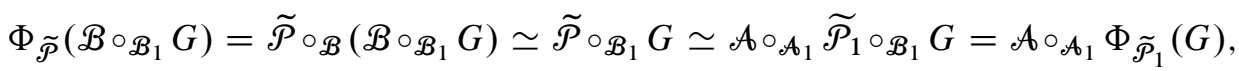

which gives the required compatibility with the induction functors.

(iii) Our assumptions imply that $\mathscr{B}$ is obtained by the proper push-forward from a bounded complex with coherent cohomologies, hence, it is a coherent sheaf itself. By part (ii), the functor $\Phi_{\widetilde{Q}}: D(\mathcal{A}$-mod $) \rightarrow D(\mathscr{B}$-mod $)$ commutes with the forgetful functors to quasi-coherent sheaves, so it preserves the condition for a complex to have coherent cohomology sheaves. By Lemma 2.2.3, we obtain a functor $D^{-}\left(\mathcal{A}-\bmod ^{\mathrm{c}}\right) \rightarrow D^{-}\left(\mathscr{B}-\bmod ^{\mathrm{c}}\right)$ commuting with forgetful functors. Since $\mathcal{Q}$ has finite Tor-dimension, it preserves boundedness of cohomology. The last assertion follows from (i).

\section{Kernel representations and derived equivalences}

In this section we develop a framework for constructing examples of the FourierMukai dual kernel algebras. More precisely, our study will focus on kernel algebras related to categories of twisted sheaves on global quotient stacks $X / G$ and their generalizations involving formal groups. The main idea is that these kernel algebras are determined by the corresponding action of $G$ on the category $D_{\mathrm{qc}}(X)$. More generally we introduce the notion of a kernel representation of a group $G$ over $X$ that can be viewed as a refined version of an action of $G$ on $D_{\mathrm{qc}}(X)$, and associate with each such kernel representation a kernel algebra over $X$. The point is that given a kernel representation of $G$ over $X$ and a derived equivalence from $X$ to $Y$, one gets a kernel representation of $G$ over $Y$. Furthermore, the associated kernel algebras will also be related by this derived equivalence. An important example of a pair of kernel algebras obtained in this way for the standard Fourier-Mukai transform of abelian varieties is given by Theorem 3.7.3. 
This section contains a lot of technical definitions and constructions. For the reader's convenience we provide here a quick informal overview. First, the kind of twisting we consider for sheaves on a quotient stack $X / G$ has to do with a notion of 1 -cocycle of $G$ with values in $\operatorname{Pic}(X)$. This is a very natural device for producing gerbes on $X / G$, similar to gerbe data of [16] (resp. presentation of a gerbe of [10]). Namely, an action of $G$ on $X$ gives rise to the so called action groupoid $\Gamma_{G} \subset X \times G \times X$ over $X$, where $\Gamma_{G}$ consists of $\left(x, g, x^{\prime}\right)$ such that $x=g x^{\prime}$. A 1-cocycle of $G$ with values in $\operatorname{Pic}(X)$ can be defined as a central extension of $\Gamma_{G}$ by $\mathbb{G}_{m}$. More explicitly, it is given by a line bundle $\mathscr{L}$ over $G \times X$ equipped with the 1-cocycle isomorphism $\mathscr{L}_{g_{1} g_{2}, x} \simeq \mathscr{L}_{g_{1}, g_{2} x} \otimes \mathscr{L}_{g_{2}, x}$ satisfying natural compatibilities. One can think of a 1-cocycle as an enrichment of a homomorphism $G \rightarrow \operatorname{Aut}(X) \ltimes \operatorname{Pic}(X)$, where the projection to $\operatorname{Aut}(X)$ corresponds to the given action of $G$ on $X$. Viewing $\operatorname{Aut}(X) \ltimes \operatorname{Pic}(X)$ as a subgroup in $\operatorname{Auteq}\left(D_{\mathrm{qc}}(X)\right)$ we are led to consider a similar enrichment of the notion of homomorphism $G \rightarrow$ $\operatorname{Auteq}\left(D_{\mathrm{qc}}(X)\right)$. This is exactly what we call a kernel representation of $G$ over $X$. When $G$ is discrete then a kernel representation is simply a rule that associates with every element $g \in G$ an object ("kernel") $V_{g} \in D_{\mathrm{qc}}(X \times X)$, compatible with composition in $G$ and with the convolution of kernels (in general, a kernel representation should be given by an object in $\left.D_{\mathrm{qc}}(X \times G \times X)\right)$.

Another useful technical notion is that of convolution algebra. Let $(s, t): M \rightarrow$ $X \times X$ be a groupoid over $X$ (in the main text we will also consider a more general case of a monoid). Then there is a natural operation of convolution for sheaves on $M$. By definition, a convolution algebra on $M$ is an algebra object in $D_{\mathrm{qc}}(M)$ with respect to the monoidal structure given by the convolution. In the case when $M=X \times X$ we recover the notion of a kernel algebra. In general, taking the push-forward of any convolution algebra on $M$ with respect to the anchor map $M \rightarrow X \times X$ one obtains a kernel algebra (see (3.2.2)). On the other hand, we show that in the case when $G$ is a finite group scheme one can associated with every kernel representation of $G$ over $X$, a certain convolution algebra on $X \times G \times X$ (see Proposition 3.2.4 and the discussion after it). Thus, we have the following sequence of constructions:

$$
\begin{gathered}
\text { 1-cocycles } \stackrel{(3.4 .5)}{\longrightarrow} \text { Kernel representations } \stackrel{\text { P. 3.2.4 }}{\longrightarrow} \text { Convolution algebras } \\
\stackrel{(3.2 .2)}{\longrightarrow} \text { Kernel algebras }
\end{gathered}
$$

The composition of the two last arrows $V \mapsto \mathcal{A}\left(V, \omega_{G / S}\right)$ associates a kernel algebra to a kernel representation $V$. The importance of this construction for us is its compatibility with derived equivalences of Fourier-Mukai type (see Proposition 3.2.5). On the other hand, we provide a more direct construction of the convolution algebra associated with a 1-cocycle $\mathscr{L}$ of $G$ with values in $\operatorname{Pic}(X)$ (see Lemma 3.4.2). This allows us to relate the category of modules over the corresponding kernel algebra $\mathcal{A}_{X}^{G}(\mathscr{L})$ with the corresponding category of twisted $G$-equivariant sheaves on $X$ (see Theorem 3.4.1 and Corollary 3.4.5). Sometimes we have to work with more flexible versions of the notions of 1-cocycle and kernel representation, called quasi-1-cocycle 
and kernel quasi-representation. The first two arrows in the above sequence still make sense for these more general notions. More importantly, we also develop a parallel story where the group $G$ is replaced with a formal group scheme.

Conventions. We denote by $S$ a fixed base scheme, and as before, all products are fibered over $S$. We also assume all schemes and formal schemes to be flat over $S$. By a formal group scheme $G$ over $S$ we mean a group object in the category of formal schemes over $S$ (flat over $S$ ). Sometimes, we will assume that a formal group scheme $G$ is of ldu-pseudofinite type (resp. ldu-pseudofinite) over $S$ - we refer to this as $l d u-p f t$ (resp. $l d u-p f$ ) over $S$.

For a field $k$ of characteristic zero we will call simply formal $k$-groups ( $k$-groupes formels in terminology of [33]), commutative formal groups $G$ over $k$ such that (i) the natural action of $\operatorname{Gal}(\bar{k} / k)$ on $G(\bar{k})$ factors through some quotient $\operatorname{Gal}\left(k^{\prime} / k\right)$ with $k^{\prime} \subset \bar{k}$ a finite Galois extension of $k$, (ii) the group $G(\bar{k})=G\left(k^{\prime}\right)$ is finitely generated, and (iii) the topological $k$-algebra $\mathcal{O}_{G, 0}$ is a quotient of $k\left[\left[t_{1}, \ldots, t_{n}\right]\right]$ for some $n$. The Cartier duality (see [23], 2.2.2, or [21], Chap. II) gives an anti-equivalence between the category of formal $k$-groups and the category of affine commutative algebraic groups over $k$. We will also use the Cartier duality for finite flat group schemes over an arbitrary base $S$.

Starting from Section 3.5, whenever formal $k$-groups are mentioned it is assumed that $S=\operatorname{Spec}(k)$.

3.1. Convolution of sheaves parametrized by formal schemes. We need a version of the convolution operation considered in Section 2.1 involving formal schemes. More precisely, formal schemes will appear only as parameter spaces so that the actual "integration" is performed only along the direction of a usual scheme. We start with the data consisting of three schemes $X, Y, Z$ and two formal schemes $P$ and $Q$. Let us denote by $D_{P, \overrightarrow{\mathrm{c}}}(X \times P \times Y) \subset D_{\mathrm{qc}}(X \times P \times Y)$ the full subcategory of objects $F$ such that for some open covering $\left(U_{i}\right)$ of $P$ the restriction of $F$ to $X \times U_{i} \times Y$ is in $D_{\overrightarrow{\mathrm{c}}}\left(X \times U_{i} \times Y\right)$. Assuming that $Y$ is proper, we can define the convolution operation

$$
D_{P, \overrightarrow{\mathrm{c}}}(X \times P \times Y) \times D_{Q, \overrightarrow{\mathrm{c}}}(Y \times Q \times Z) \rightarrow D_{P \times Q, \overrightarrow{\mathrm{c}}}(X \times P \times Q \times Z)
$$

sending $(K, L)$ to

$$
K{ }^{\circ} L:=p_{1245 *}\left(p_{123}^{*} K \otimes p_{345}^{*} L\right),
$$

where $p_{I}$ denote projections from the product $X \times P \times Y \times Q \times Z$. Note that $K{ }^{\circ} Y L$ is in $D_{P \times Q, \overrightarrow{\mathrm{c}}}$ by Proposition 1.2.2 (vi). More generally, the above operation is defined when $Y$ is not necessarily proper but $K$ is the push-forward from a closed formal subscheme proper over $X \times P$ - we will say in this case that the support of $K$ is proper over $X \times P$ (alternatively, it is enough to require the support of $L$ to be proper over $Q \times Z$ ). Using appropriate versions of the projection and base change 
formulae for formal schemes (see Theorems 1.2.3 and 1.2.4 (i)) we can easily check the following two properties of this operation:

Associativity: if $T$ (resp. $R$ ) is another scheme (resp. formal scheme) then for $M \in D_{R, \overrightarrow{\mathrm{c}}}(Z \times R \times T)$ one has a natural isomorphism

$$
\left(K \circ_{Y} L\right){ }^{\prime} Z M \simeq K \circ_{Y}\left(L \circ_{Z} M\right),
$$

provided the support of $L$ is proper over $Y \times Q$ and over $Q \times Z$.

Change of parameter spaces: if $\phi: P^{\prime} \rightarrow P$ and $\psi: Q^{\prime} \rightarrow Q$ are maps of formal schemes then we have

$\left(\left(\operatorname{id}_{X} \times \phi \times \operatorname{id}_{Y}\right)^{*} K\right) \circ_{Y}\left(\left(\operatorname{id}_{Y} \times \psi \times \operatorname{id}_{Z}\right)^{*} L\right) \simeq\left(\operatorname{id}_{X} \times \phi \times \psi \times \operatorname{id}_{Z}\right)^{*}\left(K \circ_{Y} L\right)$,

provided the support of $L$ is proper over $Q \times Z$ (or the support of $K$ is proper over $X \times P)$.

3.2. Kernel representations and convolution algebras. The following definition provides a natural framework for working with "nice" homomorphisms from a group scheme (resp. formal group scheme) to the group of autoequivalences of the derived category of quasi-coherent sheaves on a scheme.

Definition. Let $G$ be a monoid in the category of $S$-schemes, $X$ an $S$-scheme (recall that flatness over $S$ is always assumed).

(i) A kernel quasi-representation of $G$ over $X$ is an object $V \in D_{\mathrm{qc}}(X \times G \times X)$ equipped with a morphism

$$
\mu: V \circ_{X} V \rightarrow\left(\operatorname{id}_{X} \times m \times \operatorname{id}_{X}\right)^{*} V
$$

over $X \times G \times G \times X$, where $m: G \times G \rightarrow G$ is the group law, satisfying the natural associativity condition over $X \times G \times G \times G \times X$, and a morphism

$$
u: \Delta_{*} \mathcal{O}_{X} \rightarrow\left(\operatorname{id}_{X} \times e \times \operatorname{id}_{X}\right)^{*} V,
$$

where $e: S \rightarrow G$ is the unit for $G$, compatible with $\mu$ in a natural way. The associativity condition amounts to the commutativity of the following diagram:

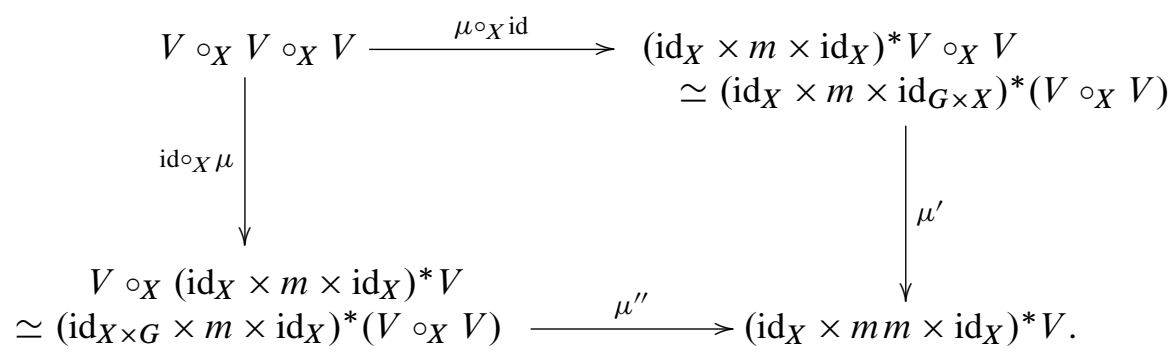


Here $m m: G^{3} \rightarrow G$ sends $\left(g_{1}, g_{2}, g_{3}\right)$ to $g_{1} g_{2} g_{3}$, the maps $\mu^{\prime}$ and $\mu^{\prime \prime}$ are induced by $\mu$, the isomorphisms are particular cases of (2.1.1). The compatibility of the unit morphism $u$ with $\mu$ means that the composition

$$
V \simeq \Delta_{*} \mathcal{O}_{X} \circ_{X} V \stackrel{{ }^{\circ} X \mathrm{id}}{\longrightarrow}\left(\operatorname{id}_{X} \times e \times \operatorname{id}_{X}\right)^{*} V \circ_{X} V \rightarrow V,
$$

where the second arrow is induced by $\left(\mathrm{id}_{X} \times e \times \mathrm{id}_{G} \times \mathrm{id}_{X}\right)^{*} \mu$ (resp. similar composition with id ${ }^{\circ} X u$ ) equals the identity map.

(ii) A kernel representation is a kernel quasi-representation such that $\mu$ and $u$ are isomorphisms.

(iii) Let $G$ be a monoid in the category of formal schemes over $S$, and let $X$ be an $S$-scheme. Then the definitions (i) and (ii) still make sense with $V \in D_{G, \overrightarrow{\mathrm{c}}}(X \times G \times X)$ provided the support of $V$ is proper over $X \times G$ and over $G \times X$ (see Section 3.1).

(iv) If $\rho: G^{\prime} \rightarrow G$ is a homomorphism of monoids and $V$ is a kernel quasirepresentation of $G$ over $X$ then we define the restriction of $V$ to $G^{\prime}$ by

$$
\rho^{*} V:=\left(\operatorname{id}_{X} \times \rho \times \operatorname{id}_{X}\right)^{*} V .
$$

This is a kernel quasi-representation of $G^{\prime}$ over $X$. The same construction works when $G$ and $G^{\prime}$ are formal group schemes provided $V \in D_{\overrightarrow{\mathrm{c}}}(X \times G \times X)$ has the support proper over $X \times G$ and over $G \times X$.

If $G$ is a discrete group then to give a kernel representation of $G$ amounts to the data of kernels $V_{g} \in D_{\mathrm{qc}}(X \times X)$ parametrized by $g \in G$ along with the isomorphisms $V_{g_{1}} \circ V_{g_{2}} \simeq V_{g_{1} g_{2}}$ satisfying the natural axioms (in particular, the functors corresponding to $V_{g}$ and $V_{g-1}$ are quasi-inverse of each other). Thus, we have an action of $G$ on $D_{\mathrm{qc}}(X)$ by the corresponding autoequivalences (in the strict sense as defined in [51]). A kernel quasi-representation in the case of a discrete group $G$ can be viewed as a $G$-graded kernel algebra $\bigoplus_{g \in G} V_{g}$. One can try to imitate this construction for general $G$ by associating with a kernel quasi-representation a kernel algebra via some kind of "integration" over $G$. Below we will show that this is possible provided one fixes an appropriate analogue of "measure" on $G$.

The passage from kernel representations to kernel algebras goes through an intermediate notion that we call a convolution algebra. We will first construct kernel algebras associated with convolution algebras and then explain the connection with kernel representations in Proposition 3.2.4.

To avoid confusion let us first give the following definition.

Definition. Let $X$ be a scheme (resp. formal scheme). We say that a scheme (resp. formal scheme) $M$ is a monoid (resp. formal monoid) over $X$ if $M$ is a monoid in the category of (formal) $X \times X$-schemes, where the monoidal structure on the latter category is given by the fibered product over $X$ (i.e., this by the composition of correspondences). Concretely, $M$ should be equipped with a morphism $M \stackrel{(s, t)}{\longrightarrow} X \times X$, 
as well as the product map $m: M \times_{X} M \rightarrow M$ and the unit map $e: X \rightarrow M$ satisfying the usual axioms. Informally, we can think of $M$ as the set of morphisms in a category that has $X$ as the set of objects (perhaps for this reason in [47] formal monoids are called "formal categories"). Note that groupoids over $X$ (considered in the theory of algebraic stacks) are examples of monoids over $X$.

Lemma 3.2.1. (i) Let $X$ be a scheme and let $M \stackrel{(s, t)}{\longrightarrow} X \times X$ be a monoid over $X$ such that the maps $s$ and $t$ from $M$ to $X$ are flat. Let $m: M \times_{X} M \rightarrow M$ and $e: X \rightarrow M$ denote the product and the unit maps. Then the category $D_{\mathrm{qc}}(M)$ has a monoidal structure given by the following convolution operation:

$$
\mathscr{F} * \mathscr{G}:=m_{*}\left(p_{1}^{*} \mathcal{F} \otimes p_{2}^{*} \mathcal{E}\right) .
$$

The sheaf $e_{*} \mathcal{O}_{X}$ is a unit object for this monoidal structure.

(ii) Now let $X$ be a formal scheme, $M \stackrel{(s, t)}{\longrightarrow} X \times X$ a formal monoid over $X$. Assume in addition that the maps $s$ and $t$ are flat and of ldu-pseudofinite type, and $m$ is ldu-quasi-compact. Then replacing $m_{*}$ with $m_{\sigma *}$ in the formula for $\mathcal{F} * \mathscr{G}$ we get a monoidal structure on the category $D_{\mathrm{qct}}(M)$ such that $e_{*} \mathcal{O}_{X}$ is still a unit object.

Proof. (i) To construct an associativity isomorphism $(\mathcal{F} * \mathscr{E}) * \mathscr{H} \simeq \mathscr{F} *(\mathscr{E} * \mathscr{H})$ we first use the base change isomorphism for the cartesian diagram

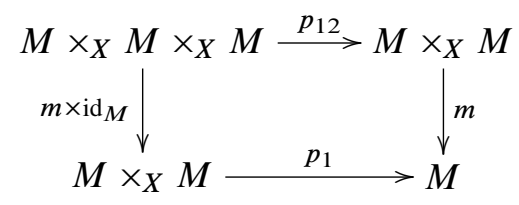

along with the projection formula for $m \times \mathrm{id}_{X}$ to get an isomorphism

$$
\begin{aligned}
(\mathscr{F} * \mathscr{G}) * \mathscr{H} & \simeq m_{*}\left(p_{1}^{*}(\mathscr{F} * \mathscr{G}) * p_{2}^{*} \mathscr{H}\right) \\
& \simeq m_{*}\left(m \times \operatorname{id}_{M}\right)_{*}\left(p_{1}^{*} \mathscr{F} \otimes p_{2}^{*} \mathcal{E} \otimes p_{3}^{*} \mathcal{H}\right) \\
& =(m m)_{*}\left(p_{1}^{*} \mathscr{F} \otimes p_{2}^{*} \mathscr{E} \otimes p_{3}^{*} \mathscr{H}\right),
\end{aligned}
$$

where $m m: M \times_{X} M \times_{X} M \rightarrow M$ is the triple composition. In a similar way we get an isomorphism

$$
\mathscr{F} *(\mathscr{H} * \mathscr{H}) \simeq(m m)_{*}\left(p_{1}^{*} \mathcal{F} \otimes p_{2}^{*} \mathscr{E} \otimes p_{3}^{*} \mathcal{H}\right) .
$$

An isomorphism $\left(e_{*} \mathcal{O}_{X}\right) * \mathscr{F} \simeq \mathscr{F}$ is obtained from the following sequence of isomorphisms

$$
\begin{aligned}
\mathcal{F} \simeq m_{*}\left(e \times \operatorname{id}_{M}\right)_{*} \mathcal{F} & \simeq m_{*}\left(\left(e \times \operatorname{id}_{M}\right)_{*} \mathcal{O}_{M} \otimes p_{2}^{*} \mathcal{F}\right) \\
& \simeq m_{*}\left(p_{1}^{*}\left(e_{*} \mathcal{O}_{X}\right) \otimes p_{2}^{*} \mathcal{F}\right)=\left(e_{*} \mathcal{O}_{X}\right) * \mathcal{F},
\end{aligned}
$$


where we use the projection formula for the map $e \times \operatorname{id}_{M}: M \rightarrow M \times_{X} M$ and the base change formula for the cartesian diagram

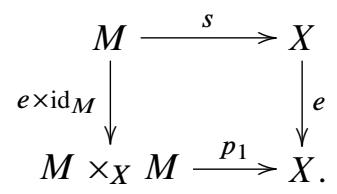

(ii) The proof is similar to (i) using Theorems 1.2.3 and 1.2.4 (ii), (iii).

Remark. Note that in the case when $M$ is a formal groupoid over $X$ the condition for $m$ to be ldu-quasi-compact in part (ii) of the above lemma follows from the assumption that $s$ is of ldu-pseudofinite type.

Definition. (i) Let $X$ be a scheme and let $M \stackrel{(s, t)}{\longrightarrow} X \times X$ a monoid over $X$ such that the maps $s$ and $t$ from $M$ to $X$ are flat. A convolution algebra on $M$ is an algebra object $\mathcal{V} \in D_{\mathrm{qc}}(M)$ with respect to the monoidal structure given by the convolution (see Lemma 3.2.1 (i)). Thus, $\mathcal{V}$ is equipped with a product morphism

and a unit morphism

$$
\mu: m_{*}\left(p_{1}^{*} \mathcal{V} \otimes p_{2}^{*} \mathcal{V}\right) \rightarrow \mathcal{V}
$$

$$
e_{*} \mathcal{O}_{X} \rightarrow \mathcal{V}
$$

satisfying the usual axioms.

(ii) Now let $X$ be a formal scheme, $M \stackrel{(s, t)}{\longrightarrow} X \times X$ a formal monoid over $X$. Assume in addition that the maps $s$ and $t$ are flat and of ldu-pseudofinite type, and $m$ is ldu-quasi-compact. A convolution algebra on $M$ is an algebra object $\mathcal{V} \in D_{\text {qct }}(M)$ with respect to the monoidal structure given in Lemma 3.2.1 (ii).

It is clear that for $M=X \times X$, where $X$ is a scheme, we get exactly the notion of a kernel algebra. On the other hand, for $M=X$ we get the notion of an algebra in $D_{\mathrm{qc}}(X)$ with respect to the tensor product. In the case when $X=S$ (the base scheme) and $M=G$ is a group scheme over $S$, the convolution algebra on $G$, is an algebra object in $D_{\mathrm{qc}}(G)$ with respect to the Pontryagin product. Similarly, if $G$ is an $l d u$-pft formal group scheme over $S$ (i.e., of ldu-pseudofinite type over $S$ ) then we have a notion of a convolution algebra structure on an object of $D_{\text {qct }}(G)$.

Lemma 3.2.2. Let $f: M \rightarrow M^{\prime}$ is the morphism of monoids over $X$ satisfying the conditions of the above definition. Then the functor $f_{*}: D_{\mathrm{qc}}(M) \rightarrow D_{\mathrm{qc}}\left(M^{\prime}\right)$ has a natural structure of monoidal functor for the convolution operations. In particular, if $\mathcal{V}$ is a convolution algebra on $M$ then $f_{*} \mathcal{V}$ has a natural structure of a convolution algebra on $M^{\prime}$. If $f^{\prime}: M^{\prime} \rightarrow M^{\prime \prime}$ is another such morphism then the natural isomorphism offunctors $\left(f^{\prime} f\right)_{*} \simeq f_{*}^{\prime} \circ f_{*}$ is compatible with the monoidal structures. 
Similar assertions hold in the case of formal monoids, where $f$ is assumed to be lduquasi-compact and $f_{*}$ is replaced with $f_{\sigma *}: D_{\mathrm{qct}}(M) \rightarrow D_{\mathrm{qct}}\left(M^{\prime}\right)$.

Proof. Let $\mathscr{F}, \mathscr{E} \in D_{\mathrm{qc}}(M)$ and set $\mathscr{F}^{\prime}=f_{*} \mathscr{F}, \mathscr{E}^{\prime}=f_{*} \mathscr{E}$. Let also $m^{\prime}: M^{\prime} \times_{X}$ $M^{\prime} \rightarrow M^{\prime}$ denote the product map for $M^{\prime}$. First let us observe that using the adjoint pair $\left((f \times f)^{*},(f \times f)_{*}\right)$ and the canonical maps $f^{*} \mathcal{F}^{\prime} \rightarrow \mathcal{F}$ and $f^{*} \mathscr{E}^{\prime} \rightarrow \mathscr{G}$ we get a morphism

$$
p_{1}^{*} \mathcal{F}^{\prime} \otimes p_{2}^{*} \mathscr{E}^{\prime} \rightarrow(f \times f)_{*}\left(p_{1}^{*} \mathcal{F} \otimes p_{2}^{*} \mathcal{E}\right)
$$

on $M^{\prime} \times M^{\prime}$. We claim that it is an isomorphism. Indeed, applying the base change formula to the cartesian square

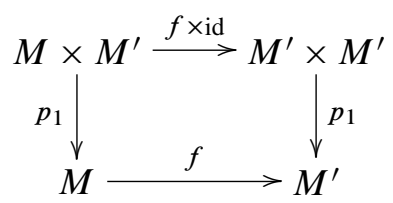

we get an isomorphism $p_{1}^{*} \mathcal{F}^{\prime} \simeq\left(f \times \mathrm{id}_{M^{\prime}}\right)_{*} p_{1}^{*} \mathcal{F}$ on $M^{\prime} \times M^{\prime}$. Together with the projection formula this gives

$$
p_{1}^{*} \mathcal{F}^{\prime} \otimes p_{2}^{*} \mathscr{E}^{\prime} \simeq\left(f \times \operatorname{id}_{M^{\prime}}\right)_{*}\left(p_{1}^{*} \mathcal{F} \otimes p_{2}^{*} \mathscr{E}^{\prime}\right) .
$$

Next, we apply the base change formula to the cartesian square

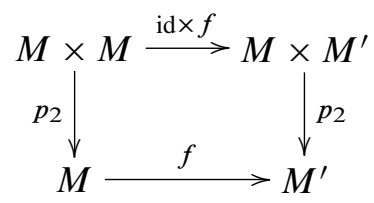

to get an isomorphism $p_{2}^{*} \mathscr{E}^{\prime} \simeq\left(\mathrm{id}_{M} \times f\right)_{*} p_{2}^{*} \mathscr{E}$. Combining it with the previous isomorphism and using the projection formula again we obtain an isomorphism

$p_{1}^{*} \mathcal{F}^{\prime} \otimes p_{2}^{*} \mathscr{E}^{\prime} \simeq\left(f \times \mathrm{id}_{M^{\prime}}\right)_{*}\left(p_{1}^{*} \mathcal{F} \otimes\left(\mathrm{id}_{M} \times f\right)_{*} p_{2}^{*} \mathscr{E}\right) \simeq(f \times f)_{*}\left(p_{1}^{*} \mathcal{F} \otimes p_{2}^{*} \mathscr{E}\right)$,

which is given by the map (3.2.1). Therefore,

$$
m_{*}^{\prime}\left(p_{1}^{*} \mathscr{F}^{\prime} \otimes p_{2}^{*} \mathscr{E}^{\prime}\right) \simeq m_{*}^{\prime}(f \times f)_{*}\left(p_{1}^{*} \mathcal{F} \otimes p_{2}^{*} \mathscr{E}\right) \simeq f_{*} m_{*}\left(p_{1}^{*} \mathscr{F} \otimes p_{2}^{*} \mathscr{E}\right)
$$

over $M^{\prime} \times_{X} M^{\prime}$, as required. To check compatibility with the associativity constraint one can use the canonical isomorphism

$$
p_{1}^{*}\left(f_{*} \mathcal{F}\right) \otimes p_{2}^{*}\left(f_{*} \mathcal{E}\right) \otimes p_{3}^{*}\left(f_{*} \mathscr{H}\right) \stackrel{\sim}{\longrightarrow}(f \times f \times f)_{*}\left(p_{1}^{*} \mathcal{F} \otimes p_{2}^{*} \mathscr{E} \otimes p_{3}^{*} \mathscr{H}\right)
$$

on $M^{\prime} \times M^{\prime} \times M^{\prime}$ for $\mathscr{F}, \mathcal{G}, \mathscr{H} \in D_{\mathrm{qc}}(M)$, defined similarly to (3.2.1). The case of formal monoids is very similar. 
For us the most important case of the above construction is when $M^{\prime}=X \times X$ and $f$ is the structure morphism $(s, t): M \rightarrow X \times X$.

Definition. Let $X$ be a scheme, $M$ a monoid (resp. formal monoid) over $X$ such that the structure maps $s, t: M \rightarrow X$ are flat (resp. flat and of ldu-pseudofinite type; in addition assume that $m$ is ldu-quasi-compact). We associate with every convolution algebra $\mathcal{V}$ over $M$ a kernel algebra over $X$ by setting

$$
\mathcal{A}_{M}(\mathcal{V})=(s, t)_{*} \mathcal{V}
$$

$\left(\right.$ resp. $\left.\mathcal{A}_{M}(\mathcal{V})=(s, t)_{\sigma *} \mathcal{V}\right)$.

The following lemma gives a sufficient condition for checking when the kernel algebra of the form $\mathcal{A}_{M}(\mathcal{V})$ is pure.

Lemma 3.2.3. Assume $X$ and $S$ are semi-separated. Let $M \stackrel{(s, t)}{\longrightarrow} X \times X$ be a monoid (resp. formal monoid) over $X$ satisfying the assumptions of the above definition, and let $\mathcal{V}$ be a convolution algebra over $X$. Assume in addition that both maps $s, t: M \rightarrow X$ are affine (resp. ldu-affine), and that $\mathcal{V}$ is a quasi-coherent sheaf (resp. torsion quasi-coherent sheaf), flat over $X$ with respect to both $s$ and $t$. Then the kernel algebra $\mathcal{A}_{M}(\mathcal{V})$ is pure.

Proof. We only need to check that $(s, t)_{*} \mathcal{V}$ (resp. $(s, t)_{\sigma *} \mathcal{V}$ ) is o-flat over $X$ with respect to both projections. But this follows immediately from Lemma 2.1.4.

If $G$ is a group scheme (resp. formal group scheme) over $S$ then we can view $X \times G \times X$ as a monoid (resp. formal monoid) over $X$ using the product on $G$ and the unit in $G$. In the following proposition we denote by $p_{i}$ (resp. $p_{i j}$ ) the projection of the product $X \times G \times X$ onto its factors (resp. double products of factors).

Proposition 3.2.4. Let $G$ be a group scheme (resp. ldu-pft formal group scheme) over $S, X$ an $S$-scheme, $V$ a kernel quasi-representation of $G$ over $X$ (recall that in the formal case we assume that $V \in D_{G, \overrightarrow{\mathrm{c}}}(X \times G \times X)$ and the support of $V$ is proper over $X \times G$ and $G \times X), P$ a convolution algebra on $G$. Then $V \otimes p_{2}^{*} P$ has a natural structure of a convolution algebra on $X \times G \times X$. Hence, we also have the corresponding kernel algebra

$$
\mathcal{A}(V, P):=\mathcal{A}_{X \times G \times X}\left(V \otimes p_{2}^{*} P\right)=p_{13 *}\left(V \otimes p_{2}^{*} P\right)
$$

over $X$ (in the formal case we should use $\left(p_{13}\right)_{\sigma *}$ ).

Proof. We will only consider the case when $G$ is a group scheme (the case of a formal group scheme is analogous). Note that the product map for $X \times G \times X$ is the composition of the projection $X \times G \times X \times G \times X \rightarrow X \times G \times G \times X$ with the 
map $\left(\operatorname{id}_{X} \times m \times \operatorname{id}_{X}\right): X \times G \times G \times X \rightarrow X \times G \times X$. Applying the projection formula for the former map we see that the convolution product on $V \otimes p_{2}^{*} P$ should correspond to a map

$$
\left(\operatorname{id}_{X} \times m \times \operatorname{id}_{X}\right)_{*}\left[\left(V \circ_{X} V\right) \otimes p_{2}^{*} P \otimes p_{3}^{*} P\right] \rightarrow V \otimes p_{2}^{*} P .
$$

To construct such map we start with the morphism

$$
\left(V \circ_{X} V\right) \otimes p_{2}^{*} P \otimes p_{3}^{*} P \rightarrow\left(\operatorname{id}_{X} \times m \times \operatorname{id}_{X}\right)^{*} V \otimes p_{2}^{*} P \otimes p_{3}^{*} P
$$

on $X \times G \times G \times X$ induced by the map $\mu: V{ }^{\circ} V \rightarrow\left(\operatorname{id}_{X} \times m \times \operatorname{id}_{X}\right)^{*} V$. Combining the projection formula for the map id $X \times m \times \operatorname{id}_{X}$ with the flat base change formula for $P \otimes P \in D_{\mathrm{qc}}(G \times G)$ and the cartesian diagram

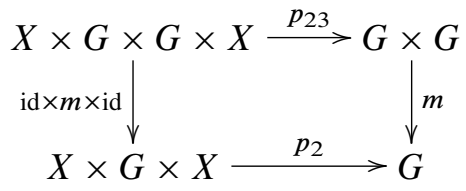

we get an isomorphism

$$
\left(\operatorname{id}_{X} \times m \times \operatorname{id}_{X}\right)_{*}\left(\left(\operatorname{id}_{X} \times m \times \operatorname{id}_{X}\right)^{*} V \otimes p_{2}^{*} P \otimes p_{3}^{*} P\right) \simeq V \otimes p_{2}^{*} m_{*}(P \otimes P) .
$$

Hence, we get the required morphism

$\mu_{V, P}:\left(\operatorname{id}_{X} \times m \times \operatorname{id}_{X}\right)_{*}\left[\left(V \circ_{X} V\right) \otimes p_{2}^{*} P \otimes p_{3}^{*} P\right] \rightarrow V \otimes p_{2}^{*} m_{*}(P \otimes P) \rightarrow V \otimes p_{2}^{*} P$

over $X \times G \times X$, where the second arrow is induced by the convolution algebra structure on $P$. The associativity is checked as follows. Let us consider the following diagram with cartesian squares

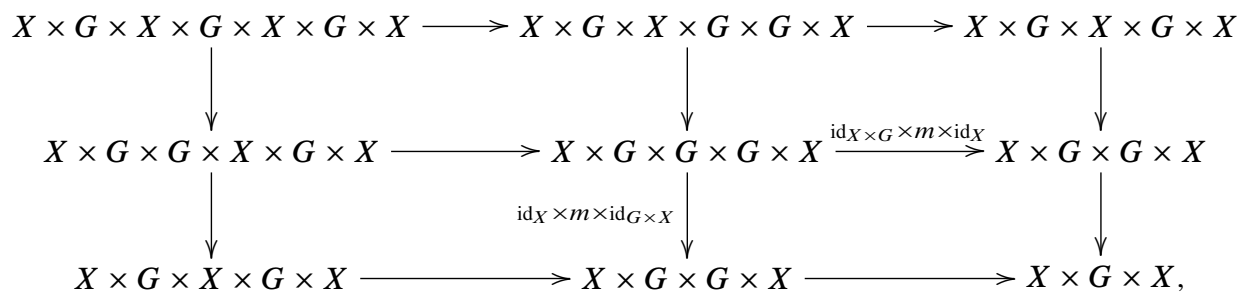

where all the arrows are either projections omitting one of the factors $X$, or maps induced by the product in two consecutive factors $G$. For each vertex $v$ in this diagram let $X(v)=X \times G^{n_{1}} \times X \times G^{n_{2}} \times \cdots$ denote the corresponding scheme. We have a natural object $W(v) \in D(X(v))$ defined as follows: let $m(v): X(v) \rightarrow X \times G \times$ $X \times G \cdots \times X$ denote the map induced by the product maps $G^{n_{1}} \rightarrow G, G^{n_{2}} \rightarrow G$, etc. Then $W(v)$ is given by the (derived) tensor product of pull-backs of $P$ from all the factors of $G$ with the pull-back by $m(v)$ of the object $p_{123}^{*} V \otimes p_{345}^{*} V \otimes \cdots \epsilon$ $D(X \times G \times X \times G \cdots \times X)$. Next, for every arrow $e: v \rightarrow v^{\prime}$ let $f(e): X(v) \rightarrow X\left(v^{\prime}\right)$ 
be the corresponding morphism. Then we have natural map $f(e)_{*} W(v) \rightarrow W\left(v^{\prime}\right)$ induced either by $\mu$ (in the case when $f(e)$ is the projection omitting one of the factors $X$ ), or by the convolution product on $P$ (when $f(e)$ is induced by $m$ ). Furthermore, we claim that for each of the four small cartesian squares of our diagram

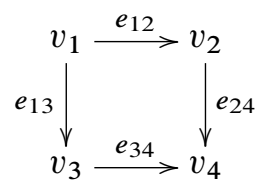

the induced morphisms

$$
f\left(e_{24} \circ e_{12}\right)_{*} X\left(v_{1}\right) \rightarrow f\left(e_{24}\right)_{*} f\left(e_{12}\right)_{*} X\left(v_{1}\right) \rightarrow f\left(e_{24}\right)_{*} X\left(v_{2}\right) \rightarrow X\left(v_{4}\right)
$$

and

$$
f\left(e_{34} \circ e_{13}\right)_{*} X\left(v_{1}\right) \rightarrow f\left(e_{34}\right)_{*} f\left(e_{13}\right)_{*} X\left(v_{1}\right) \rightarrow f\left(e_{34}\right)_{*} X\left(v_{3}\right) \rightarrow X\left(v_{4}\right)
$$

are the same. Indeed, for the upper left (resp. lower right) square this amounts to the associativity of $\mu$ (resp. of the convolution product on $P$ ). For the remaining two squares this can be deduced from the base change formula. Now the required associativity of $\mu_{P}$ follows from the similar compatibility applied to the ambient square in the big diagram above.

The unit for $V \otimes p_{2}^{*} P$ is given by the composition

$$
\begin{aligned}
\left(\operatorname{id}_{X} \times e \times \operatorname{id}_{X}\right)_{*} \Delta_{*} \mathcal{O}_{X} & \stackrel{u}{\rightarrow}\left(\operatorname{id}_{X} \times e \times \operatorname{id}_{X}\right)_{*}\left(\operatorname{id}_{X} \times e \times \operatorname{id}_{X}\right)^{*} V \\
& \simeq V \otimes\left(\operatorname{id}_{X} \times e \times \operatorname{id}_{X}\right)_{*} \mathcal{O}_{X \times X} \rightarrow V \otimes p_{2}^{*} P,
\end{aligned}
$$

where the second arrow is induced by the unit for $P$.

Assume that $G$ is a finite group scheme (resp. locally nicely ind-finite formal group scheme) over $S$. Let us consider the dualizing sheaf $\omega_{G / S}:=\pi^{!} \mathcal{O}_{S}$, where $\pi: G \rightarrow S$ is the projection (in the formal case we use the functor $\pi^{!}$from Proposition 1.2.5 (ii)). It is easy to see that $\omega_{G / S}$ has a natural structure of a convolution algebra on $G$ (see Theorem 3.3.1 below for a more general construction). Thus, in this case we can associate with any quasi-kernel representation $V$ of $G$ the kernel algebra $\mathcal{A}\left(V, \omega_{G / S}\right)$ over $X$.

For us the most useful property of kernel (quasi-)representation is that they can be carried over under derived equivalences in a way compatible with the corresponding operation on kernel algebras. This follows from the following more general statement.

Proposition 3.2.5. Let $X$ and $Y$ be $S$-schemes, and let $(\mathcal{P}, \mathcal{Q}, \alpha, \beta)$ be adjoint kernel data, where $\mathcal{P} \in D_{\mathrm{qc}}(X \times Y)$ and $\mathcal{Q} \in D_{\mathrm{qc}}(Y \times X)$ (see Section 2.5). Let $G$ be a group scheme over $S$, and let $V$ be a kernel quasi-representation of $G$ over $X$. Then ${ }_{Q}{ }^{\circ} V \circ_{X} \mathcal{P}$ has a natural structure of a kernel quasi-representation of $G$ over $Y$. This correspondence is compatible with the restriction under a homomorphism of 
group schemes $G^{\prime} \rightarrow G$. Furthermore, if $P$ is a convolution algebra on $G$ then we have an isomorphism of kernel algebras over $Y$ :

$$
\mathcal{A}\left(\left(\mathcal{Q} \circ_{X} V \circ_{X} \mathcal{P}\right), P\right) \simeq \mathcal{Q} \circ_{X} \mathcal{A}(V, P) \circ_{X} \mathcal{P} .
$$

Similar assertions hold if $G$ is a formal group scheme over $S, X$ and $Y$ are proper over $S, V \in D_{G, \overrightarrow{\mathrm{c}}}(X \times G \times X)$ (in the analog of the last assertion we should require $G$ to be ldu-pft over $S$ in order to consider a convolution algebra over it).

Proof. Using the associativity of the o-product, the map $\alpha: \mathcal{P} \circ_{Y} \mathcal{Q} \rightarrow \Delta_{*} \mathcal{O}_{X}$ and the product map $\mu$ for $V$ we obtain a morphism

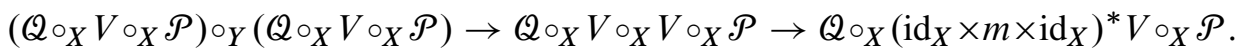

By Lemma 2.1.2(ii), the target of this map is naturally isomorphic to $\operatorname{(id}_{Y} \times m \times$ $\left.\operatorname{id}_{Y}\right)^{*}\left(\mathcal{Q}_{{ }_{X}} V{ }{ }_{X} \mathcal{P}\right)$. This gives a product map for $\mathcal{Q}{ }^{\circ} V{ }^{\circ} \mathcal{P}$. On the other hand, by Lemma 2.1.2 (iii), we have

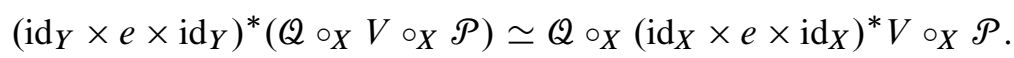

Hence, the unit for $V$ together with the map $\beta: \Delta_{*} \mathcal{O}_{Y} \rightarrow \mathcal{Q}{ }^{\circ} \mathcal{P}$ induce a unit for $\mathcal{Q} \circ_{X} V \circ_{X} \mathcal{P}$. The isomorphism (3.2.3) is obtained using the associativity of the o-product:

$$
\begin{aligned}
\mathcal{A}\left(\left(\mathcal{Q} \circ_{X} V \circ_{X} \mathcal{P}\right), P\right) & =\left(\mathcal{Q} \circ_{X} V \circ_{X} \mathcal{P}\right){ }^{\circ}{ }_{G \times X}\left(P \square \Delta_{*} \mathcal{O}_{X}\right) \\
& \simeq \mathcal{Q} \circ_{X} V{ }^{\circ}{ }_{G \times X}\left(\Delta_{*} \mathcal{O}_{G} \square \mathcal{P}\right){ }^{\circ}{ }_{G \times X}\left(P \square \Delta_{*} \mathcal{O}_{X}\right) \\
& \simeq \mathcal{Q} \circ_{X} V{ }^{\circ}{ }_{G \times X}\left(P \square \Delta_{*} \mathcal{O}_{X}\right){ }_{G \times X}\left(\Delta_{*} \mathcal{O}_{G} \square \mathcal{P}\right) \\
& \simeq \mathcal{Q} \circ_{X}\left(V{ }^{\circ}{ }_{G \times X}\left(P \square \Delta_{*} \mathcal{O}_{X}\right)\right){ }^{\circ} \mathcal{P} .
\end{aligned}
$$

3.3. Equivariant sheaves as modules over kernel algebras. We are going to show that in the situation when a finite group scheme (resp. locally nicely ind-finite formal group scheme) acts on a scheme $X$ there is a natural way to construct a kernel algebra on $X$ that captures the corresponding category of equivariant sheaves. In fact, we can deal with a more general situation of a groupoid over $X$.

Theorem 3.3.1. (i) Let $(s, t): M \rightarrow X \times X$ be a groupoid over $X$. Assume that the maps $s$ and $t$ are finite and flat. Then the relative dualizing sheaf $\omega_{s}=s^{!} \mathcal{O}_{X}$ has a natural structure of a convolution algebra on $M$. Its push-forward to $X \times X$ is a pure kernel algebra $\mathcal{A}_{M}=\mathcal{A}_{M}\left(\omega_{s}\right)$ over $X$. The category of $\mathcal{A}_{M}$-modules is equivalent to the category of $M$-equivariant quasi-coherent sheaves on $X$. By definition, these are quasi-coherent sheaves $F$ on $X$ equipped with an isomorphism $\alpha: t^{*} F \rightarrow s^{*} F$ such that

$$
m^{*} \alpha=p_{1}^{*} \alpha \circ p_{2}^{*} \alpha, \quad e^{*} \alpha=\operatorname{id}_{F},
$$


where $m, p_{1}, p_{2}: M \times_{X} M \rightarrow M$ are the product and the projections, $e: X \rightarrow M$ is the unit map.

(ii) The above statements hold also in the case when $M$ is a formal groupoid over $X$ with the following changes. We assume that locally over $S$ each connected component of $M$ is affine over $X$ with respect to both $s$ and $t$ and can be presented as the inductive limit of a system of closed subschemes $M_{n} \subset M_{n+1} \subset \cdots \subset M$ such that each $M_{n}$ is finite and flat over $X$ with respect to both $s$ and $t$. We set $\omega_{s}=s^{!} \mathcal{O}_{X}$ (see Proposition 1.2.5 (ii)). The definition of an $M$-equivariant structure on $F$ should be modified as follows: it is given by a map $\alpha \in \widehat{\operatorname{Hom}}\left(t^{*} F, s^{*} F\right)$, and the relations (3.3.1) are imposed using $\widehat{\mathrm{Hom}}$-spaces (see (1.2.1)).

Proof. Part (i) is a particular case of part (ii), so it is enough to consider the case of a formal groupoid. Our assumptions imply that both morphisms $s, t: M \rightarrow X$ (and hence $\left.p_{1}, p_{2}: M \times_{X} M \rightarrow M\right)$ are nicely ind-finite. Since $M$ is a groupoid, the map $m: M \times_{X} M \rightarrow M$ differs from the projection $p_{1}$ by an automorphism of $m$. Therefore, $m$ is also nicely ind-finite. Thus, we can use the functor $m$ ! defined in Proposition 1.2.5. In particular, we have the isomorphism

$$
m ! \omega_{s}=m ! s^{!} \mathcal{O}_{S} \simeq(s \circ m)^{!} \mathcal{O}_{S}=\left(s \circ p_{1}\right)^{!} \mathcal{O}_{S} \simeq p_{1}^{!} \omega_{s}
$$

on $M \times_{X} M$. Now we define the convolution algebra structure on $\omega_{s}$ as the map corresponding by adjunction to the map

$$
p_{1}^{*} \omega_{s} \otimes p_{2}^{*} \omega_{s} \rightarrow p_{1}^{!} \omega_{s} \simeq m^{!} \omega_{s}
$$

on $M \times_{X} M$, where the arrow is given by the isomorphism (1.2.14). The associativity of this product reduces to the commutativity of the diagram in Corollary 1.2.6 for $M \times_{X} M \times_{X} M$ with $F=\omega_{S}$, along with some easier compatibilities. The unit $e_{*} \mathcal{O}_{S} \rightarrow \omega_{s}=s^{!} \mathcal{O}_{S}$ corresponds by adjunction to the isomorphism $s ! e_{*} \mathcal{O}_{S} \simeq \mathcal{O}_{S}$.

Using Lemma 3.2.3 we see that $\mathcal{A}_{M}$ is pure. Indeed, both $s$ and $t$ are ldu-affine, so we only need to check flatness of $\omega_{s}$ over $X$ with respect to both $s$ and $t$. But this follows immediately from the construction of Proposition 1.2.5, since $\omega_{s}$ is the inductive limit of line bundles over $M_{n}$.

By Proposition 1.2.5 (iv), an element $\alpha \in \widehat{\operatorname{Hom}}\left(t^{*} F, s^{*} F\right)$ required in the definition of an $M$-equivariant sheaf $F$ on $X$ is the same as a morphism

$$
t^{*} F \otimes \omega_{s} \rightarrow s^{*} F \otimes \omega_{s} \stackrel{\sim}{\longrightarrow} s^{!} F,
$$

where the last arrow is an isomorphism by Proposition 1.2.5 (ii). By adjunction this is the same as a morphism

$$
s_{!}\left(t^{*} F \otimes \omega_{s}\right) \rightarrow F .
$$

By the projection formula, the source of this map is isomorphic to $\mathcal{A}_{M}{ }^{\circ} X F$. Conversely, given a morphism $\mathcal{A}_{M}{ }^{\circ} X F \rightarrow F$, reversing the above procedure we get a map $t^{*} F \otimes \omega_{s} \rightarrow s^{*} F \otimes \omega_{s}$, or equivalently, an element $\alpha \in \widehat{\operatorname{Hom}}\left(t^{*} F, s^{*} F\right)$. 
We have to check that the module axiom for $F$ is equivalent to the conditions (3.3.1). Using the projection formula and the base change formula we get an isomorphism

$$
\mathcal{A}_{M} \circ_{X} \mathcal{A}_{M} \circ_{X} F \simeq\left(s p_{1}\right)_{!}\left(\left(t p_{2}\right)^{*} F \otimes p_{1}^{*} \omega_{s} \otimes p_{2}^{*} \omega_{s}\right),
$$

where $p_{1}, p_{2}: M \times_{X} M \rightarrow M$ are the projections. Furthermore, the map

$$
\mathcal{A}_{M}{ }^{\circ}{ }_{X} \mathcal{A}_{M}{ }^{\circ}{ }_{X} F \rightarrow \mathcal{A}_{M}{ }^{\circ} X \rightarrow F
$$

obtained from two action maps $\mathcal{A}_{M}{ }^{\circ} X F \rightarrow F$ corresponds by adjunction to the map

$$
\begin{aligned}
\left(t p_{2}\right)^{*} F \otimes p_{1}^{*} \omega_{s} \otimes p_{2}^{*} \omega_{s} & \rightarrow p_{2}^{*}\left(s^{*} F \otimes \omega_{s}\right) \otimes p_{1}^{*} \omega_{s} \\
& \rightarrow p_{1}^{*}\left(t^{*} F \otimes \omega_{s}\right) \otimes p_{1}^{!} \mathcal{O}_{M} \\
& \rightarrow p_{1}^{!}\left(t^{*} F \otimes \omega_{s}\right) \rightarrow p_{1}^{!} s^{!} F \simeq\left(s p_{1}\right)^{!} F,
\end{aligned}
$$

where the first and the last arrows are induced by (3.3.4), the second arrow is induced by the isomorphism $p_{2}^{*} \omega_{s} \simeq p_{1}^{!} \mathcal{O}_{M}$ (using Proposition 1.2.5 (iii)). Note that we have

$$
\left(s p_{1}\right)^{!} \mathcal{O}_{X} \simeq p_{1}^{*} \omega_{s} \otimes p_{1}^{!} \mathcal{O}_{M} \simeq p_{1}^{*} \omega_{s} \otimes p_{2}^{*} \omega_{s},
$$

hence $\left(s p_{1}\right)^{!} F \simeq\left(s p_{1}\right)^{*} F \otimes p_{1}^{*} \omega_{s} \otimes p_{2}^{*} \omega_{s}$. Now the first relation in (3.3.1) is equivalent to the condition that under this isomorphism the map (3.3.5) coincides with the map

$$
(t m)^{*} F \otimes p_{1}^{*} \omega_{s} \otimes p_{2}^{*} \omega_{s} \rightarrow(s m)^{*} F \otimes p_{1}^{*} \omega_{s} \otimes p_{2}^{*} \omega_{s}
$$

induced by $\alpha$. Indeed, this can be deduced easily from the commutativity of the diagram in Proposition 1.2.5 (iii) for the composition of $s$ with $p_{1}$ and from the isomorphism of Proposition 1.2.5 (iv) for the composed map $s p_{1}$. On the other hand, the module axiom requires that (3.3.5) coincides with the map

$$
\begin{aligned}
(t m)^{*} F \otimes p_{1}^{*} \omega_{s} \otimes p_{2}^{*} \omega_{s} & \rightarrow m^{*} t^{*} F \otimes m^{!} \omega_{s} \\
& \rightarrow m^{*}\left(t^{*} F \otimes \omega_{s}\right) \otimes m^{!} \mathcal{O}_{M} \\
& \rightarrow m^{!}\left(t^{*} F \otimes \omega_{s}\right) \rightarrow m^{!} s^{!} F \simeq(s m)^{!} F,
\end{aligned}
$$

where the first arrow is induced by (3.3.3), the second by the isomorphism $m^{!} \omega_{s} \simeq$ $m^{*} \omega_{s} \otimes m^{!} \mathcal{O}_{M}$, the third by the isomorphism (1.2.7), and the last by (3.3.4). Using the isomorphism $(\mathrm{sm})^{!} F \simeq(\mathrm{sm})^{*} F \otimes p_{1}^{*} \omega_{s} \otimes p_{2}^{*} \omega_{s}$ and the commutativity of the diagram in Proposition 1.2.5 (iii) for the composition of $s$ with $m$ one can check that the maps (3.3.7) and (3.3.6) are the same, which finishes the proof.

Example. In the case when $S=\operatorname{Spec}(k)$, where $k$ is a field of characteristic zero, and $M$ is the infinitesimal groupoid corresponding to a Lie algebroid $L$ over $X$, the kernel algebra $\mathcal{A}_{M}$ is isomorphic to the universal enveloping algebra of $L$ viewed as a $D$-algebra (see [9], 1.4.15). Thus, the above theorem reduces in this case to the interpretation of $L$-modules as $M$-equivariant sheaves on $X$. 
Assume that a finite group scheme $G$ acts on $X$. Then we have the corresponding action groupoid

$$
\Gamma_{G}=\{(g x, g, x) \mid g \in G, x \in X\} \subset X \times G \times X,
$$

where the maps $s, t: \Gamma_{G} \rightarrow X$ are induced by $p_{1}$ and $p_{3}$ (projections from $X \times G \times X$ ). Hence, by the above theorem we have the corresponding pure kernel algebra over $X$,

$$
\mathcal{A}_{X}^{G}:=\mathcal{A}_{\Gamma_{G}}=p_{13 *}\left(p_{2}^{*} \omega_{G / S}\right)
$$

Now let $G$ be a formal group scheme, locally nicely ind-finite over $S$. We define a $G$-equivariant structure on a quasi-coherent sheaf $F$ on $X$ as an isomorphism in the sense of Hom-spaces of two pull-backs of $F$ to $G \times X$ (via the projection and the action map $G_{n} \times X \rightarrow X$ ), satisfying the usual compatibility conditions (still in Hom-spaces). Then we can apply Theorem 3.3.1 (ii) to the formal groupoid $\Gamma_{G}$ to define the pure kernel algebra $\mathcal{A}_{X}^{G}$ by the same formula as above (but with $p_{13 *}$ replaced by $p_{13}$ !). Theorem 3.3.1 implies the following result.

Corollary 3.3.2. Let $G$ be finite group scheme (resp. locally nicely ind-finite formal group scheme) acting on a scheme $X$. Then the category of $G$-equivariant quasicoherent sheaves on $X$ is equivalent to the category of $\mathcal{A}_{X}^{G}$-modules.

Note that in the above situation the coherent sheaf $\mathcal{O}_{\Gamma_{G}}$ on $X \times G \times X$ has a natural structure of a kernel representation of $G$ over $X$. Hence, using the construction of Proposition 3.2.4 we can associate with this kernel representation the kernel algebra $\mathcal{A}\left(\mathcal{O}_{\Gamma_{G}}, \omega_{G / S}\right)$. It is easy to see that it is isomorphic to $\mathcal{A}_{X}^{G}$ (see Lemma 3.4.4 for a more general statement).

3.4. Twisting geometric actions by 1-cocycles. Recall that if $\mathcal{X}$ is an algebraic stack then a cohomology class $e \in H^{2}\left(\mathcal{X}, \mathbb{G}_{m}\right)$ (i.e., a $\mathbb{G}_{m}$-gerbe over $\mathcal{X}$ ) allows to define a category of twisted quasi-coherent sheaves on $\mathcal{X}$ (see e.g. Sec. 2 of [34]). We will show that some of the twisted categories of sheaves for the global quotient stack $\mathcal{X}=[X / G]$ are related to kernel representations of $G$ over $X$ of a special kind. It is convenient to describe $\mathbb{G}_{m}$-gerbes over a stack using certain data over its groupoid presentation. The relevant notion of a 1-cocycle on a groupoid generalizes the well known description of gerbes using open coverings and line bundles on pairwise intersections (see Sec. 1 of [16], and also [31], [10]).

Definition. Let $X$ be a scheme, $(s, t): M \rightarrow X \times X$ a groupoid (resp. formal groupoid) over $X$. A 1-cocycle on $M$ is a line bundle $\mathscr{L}$ on $M$ equipped with an isomorphism

$$
p_{1}^{*} \mathscr{L} \otimes p_{2}^{*} \mathscr{L} \rightarrow m^{*} \mathscr{L}
$$


on $M \times_{X} M$, where $m, p_{1}, p_{2}: M \times_{X} M \rightarrow M$ are the product and the projection maps, such that the following diagram on $M \times_{X} M \times_{X} M$ ) is commutative:

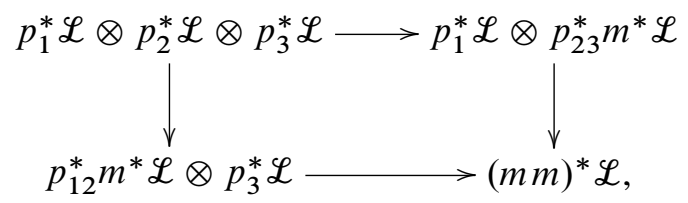

where $m m: M \times_{X} M \times_{X} M \rightarrow M$ is the composed product map.

In the case when $M=\Gamma_{G}=G \times X$ is the action groupoid associated with an action of a group scheme (resp. formal group scheme) $G$ on $X$ we will also say that $\mathscr{L}$ is a 1 -cocycle of $G$ with values in $\operatorname{Pic}(X)$.

Let $e: X \rightarrow M$ be the unit. Pulling back (3.4.1) via $e \times e: X \rightarrow M \times_{X} M$ we obtain an isomorphism

$$
\mathcal{O}_{X} \rightarrow e^{*} \mathscr{L}
$$

on $X$. Using the compatibility diagram above one can check that this isomorphism is compatible with the restrictions of maps (3.4.1) to $M$ via the maps $e \times \operatorname{id}_{M}: M \rightarrow$ $M \times_{X} M$ and $\operatorname{id}_{M} \times e: M \rightarrow M \times_{X} M$.

Let $\tilde{M} \rightarrow M$ be the $\mathbb{G}_{m}$-torsor associated with $\mathscr{L}$. Then isomorphisms (3.4.1) and (3.4.3) give rise to a groupoid structure on $\widetilde{M}$ over $X$ such that $\widetilde{M}$ is a central extension of $M$ by the group $\mathbb{G}_{m}$ acting trivially on $X$. Conversely, every such an extension arises from a 1-cocycle structure on $\mathscr{L}$.

Examples. 1. In the case when $X \rightarrow Y$ is an fppf map of schemes (or a presentation of an algebraic stack $Y$ ) and $M=X \times_{Y} X$ is the corresponding groupoid, the notion of a 1-cocycle on $M$ is an algebraic version of gerbe data of [16] (resp. a presentation of a gerbe of [10]). Let us show how to construct a $\mathbb{G}_{m}$-gerbe $\mathscr{E}_{\mathscr{L}}$ on $Y$ corresponding to a 1-cocycle $\mathscr{L}$ on $M=X \times_{Y} X$. For an open $U \rightarrow Y$ (in flat topology) let us consider $E=X \times_{Y} U$, and let $\mathscr{L}_{U}$ denote the pull-back of $\mathscr{L}$ to $E \times_{U} E$. Then $\mathscr{G}_{\mathscr{L}}(U)$ is the category of line bundles $\xi$ over $E$ equipped with an isomorphism

$$
p_{1}^{*} \xi \simeq p_{2}^{*} \xi \otimes \mathscr{L}_{U}
$$

over $E \times_{U} E$, satisfying the obvious compatibility on $E \times_{U} E \times_{U} E$. Note that the pull-back of this gerbe to $X$ is trivialized, namely, the 1-cocycle $\mathscr{L}$ itself may be viewed as an object of $\mathscr{E}_{\mathscr{L}}(X)$. Conversely, a $\mathbb{G}_{m}$-gerbe on $Y$ equipped with a trivialization of its pull-back to $X$ gives rise to a 1-cocycle on $X \times_{Y} X$ : one simply looks at the difference of two trivializations of our gerbe to $X \times_{Y} X$ induced by two projections to $X$. It is easy to see in this way one gets an equivalence between the category of 1-cocycles on $X \times_{Y} X$ and the category of $\mathbb{G}_{m}$-gerbes on $Y$ with trivialized pull-back to $X$. For differentiable stacks and $S^{1}$-gerbes a similar picture is considered in Sec. 4.2 of [6] (using the equivalent language of central extensions). 
Let us observe also that a 1-cocycle $\mathscr{L}$ on $X \times_{Y} X$ gives rise to an element of the relative Picard group Pic $(X / Y)$ defined by Grothendieck in [26], and in the situation considered in [27] the corresponding element of the cohomological Brauer group is the class of the gerbe $e_{\mathscr{L}}$.

2. In the case when $G$ is discrete a 1-cocycle of $G$ with values in $\operatorname{Pic}(X)$ induces a 1-cocycle of $G$ with values in the abelian group $\operatorname{Pic}(X)$ in the usual sense, where $\operatorname{Pic}(X)$ is viewed as a $G$-module. The obstacle to lifting a usual 1-cocycle of $G$ with values in $\operatorname{Pic}(X)$ to $\operatorname{Pic}(X)$ is a certain cohomology class in $H^{3}\left(G, H^{0}\left(X, \mathcal{O}^{*}\right)\right)$ that measures the defect for commutativity of the diagram (3.4.2). Note also that to give a 1-cocycle of $G$ with values in $\operatorname{Pic}(X)$ is equivalent to lifting the homomorphism $G \rightarrow \operatorname{Aut}(X)$ to a group homomorphism $G \rightarrow \operatorname{Aut}(X) \ltimes \operatorname{Pic}(X)$. On the other hand, we will show below that a 1-cocycle with values in $\operatorname{Pic}(X)$ gives rise to a kernel representation of $G$ over $X$. Thus, if we think of the notion of kernel representation as a refinement of a homomorphism $G \rightarrow \operatorname{Auteq}\left(D_{\mathrm{qc}}(X)\right)$ then 1-cocycles with values in $\operatorname{Pic}(X)$ correspond to homomorphisms that factor through $\operatorname{Aut}(X) \ltimes \operatorname{Pic}(X)$.

3. The holomorphic analog of the above notion can be used to study holomorphic gerbes on quotients by actions of discrete groups. Taking $X$ to be a complex vector space and $G$ a lattice acting on $X$ by translations, one gets a construction of gerbes on complex tori. It is easy to see that all gerbes on complex tori appear in this way. Furthermore, due to triviality of the relevant line bundles, these gerbes are in fact described by 2-cocycles of $G$ with values in the group of invertible functions on $X$. An explicit cocycle representative for each equivalence class of gerbes was constructed in [11] (these representatives are similar to Appell-Humbert 1-cocycles describing holomorphic line bundles on complex tori).

4. In the case when $M$ is the completion of $X \times X$ along the diagonal $\Delta \subset X \times X$, where $X$ is a smooth scheme over $k, 1$-cocycles on $M$ correspond to algebras of twisted differential operators on $X$ (see [7]). Indeed, as explained in [7], the latter rings arise as universal enveloping algebras of Picard algebroids on $X$, i.e., central extensions of the tangent algebroid of $X$ by $\mathcal{O}_{X}$. On the other hand, 1-cocycles on $M$ can be viewed as central extensions of $M$ by the 1-dimensional formal group over $k$ (acting trivially on $X$ ). It remains to apply the correspondence between Lie algebroids and formal groupoids over $X$ (see [9], 1.4.14, 1.4.15).

Given the connection of 1-cocycles with gerbes outlined above we should expect that they define twisted versions of the categories of equivariant sheaves.

Definition. Let $(s, t): M \rightarrow X \times X$ be a groupoid over $X, \mathscr{L}$ a 1-cocycle on $M$. An $\mathscr{L}$-twisted $M$-equivariant quasi-coherent sheaf on $X$ is a quasi-coherent sheaf $F$ on $X$ equipped with an isomorphism $\alpha: \mathscr{L} \otimes t^{*} F \rightarrow s^{*} F$ such that equations (3.3.1) are satisfied (these equations make sense because of the isomorphism $m^{*} \mathscr{L} \simeq p_{1}^{*} \mathscr{L} \otimes p_{2}^{*} \mathscr{L}$ and the trivialization of $e^{*} \mathscr{L}$ ). Morphisms between $\mathscr{L}$-twisted $M$-equivariant sheaves are defined in a natural way. Note that if we use the corresponding extension $\tilde{M}$ of $M$ by $\mathbb{G}_{m}$ then an $\mathscr{L}$-twisted $M$-equivariant sheaf can be 
thought of as a $\tilde{M}$-equivariant sheaf on $X$ on which $\mathbb{G}_{m}$ acts via the natural character.

The above definition can also be applied with appropriate changes to the case when $M$ is a formal groupoid over $X$, satisfying the conditions of Theorem 3.3.1 (ii): the morphism $\alpha$ and the compatibilities should be formulated using $\widehat{\text { Hom }}$-spaces (see (1.2.1)).

If $\mathscr{L}$ and $\mathscr{L}^{\prime}$ are 1 -cocycles on a groupoid $(s, t): M \rightarrow X \times X$ then there is a natural structure of a 1-cocycle on the tensor product of line bundles $\mathscr{L} \otimes \mathscr{L}^{\prime}$.

Definition. . With a line bundle $L$ on $X$ we associate the line bundle $\delta L$ on $M$ by $\delta L=s^{*} L \otimes t^{*} L^{-1}$. Note that $\delta L$ has a natural structure of a 1-cocycle on $M$ which we call a coboundary 1-cocycle. If $\mathscr{L}$ and $\mathscr{L}^{\prime}$ are 1-cocycles on $M$ equipped with an isomorphism $\mathscr{L}^{\prime} \simeq \mathscr{L} \otimes \delta L$ of 1-cocycles for some line bundle $L$ on $X$, then we say that the 1 -cocycles $\mathscr{L}$ and $\mathscr{L}^{\prime}$ are cohomologous.

In the situation of Example 1 above cohomologous 1-cocycles lead to equivalent gerbes. In general, corresponding categories of twisted $M$-equivalent sheaves on $X$ will be equivalent. Indeed, if $F$ is an $\mathscr{L}$-twisted $M$-equivariant sheaf on $X$ then $F \otimes L$ has a natural structure of an $\mathscr{L} \otimes \delta L$-twisted $M$-equivariant sheaf .

We have the following analog of Theorem 3.3.1 in the twisted case.

Theorem 3.4.1. Let $(s, t): M \rightarrow X$ be a formal groupoid over $X$ satisfying the conditions of Theorem 3.3.1 (ii), and let $\mathscr{L}$ be a 1-cocycle on $M$. Then the sheaf $\mathscr{L} \otimes \omega_{s}$ has a natural structure of a convolution algebra on $M$. Its push-forward to $X \times X$ is a pure kernel algebra $\mathcal{A}_{M}\left(\mathscr{L} \otimes \omega_{s}\right)$ over $X$. The category of $\mathcal{A}_{M}\left(\mathscr{L} \otimes \omega_{s}\right)$ modules is equivalent to the category of $\mathscr{L}$-twisted $M$-equivariant quasi-coherent sheaves on $X$.

The proof is similar to that of Theorem 3.3.1. Note that the convolution algebra structure on $\mathscr{L} \otimes \omega_{s}$ is induced by the maps (3.3.3) and (3.4.1) along with the natural map $m^{*} \mathscr{L} \otimes m^{!} \omega_{s} \rightarrow m^{!}\left(\mathscr{L} \times \omega_{s}\right)$ (see Lemma 3.4 .2 below for a more general construction).

The following generalization of the notion of 1-cocycle leaves only those features of this notion that are necessary to get a convolution algebra on $M$.

Definition. Let $(s, t): M \rightarrow X \times X$ be a formal groupoid over a scheme $X$. A quasi-1-cocycle on $M$ is an object $\mathcal{F}_{\mathcal{F}} \in D_{\mathrm{qc}}(M)$ equipped with a morphism $\alpha: p_{1}^{*} M \otimes p_{2}^{*} M \rightarrow m^{*} M$ on $M \times_{X} M$ as in the definition of 1-cocycle (however, this morphism is not required to be an isomorphism) and subject to the same constraint on $G \times G \times G \times X$. In addition, $\mathcal{F}$ should be equipped with a map $\mathcal{O}_{X} \rightarrow e^{*} \mathcal{F}$ (which we call a unit for $\mathcal{F}$ ), compatible with the pull-backs of $\alpha$ to $M$ via $e \times \operatorname{id}_{M}$ and $\operatorname{id}_{M} \times e$.

In the case when $M=\Gamma_{G}=G \times X$ is an action groupoid associated with an action of a group scheme (resp. formal group scheme) $G$ on $X$ we will also say that $\mathcal{F}_{\text {is }}$ a a quasi-1-cocycle of $G$ with values in $D_{\mathrm{qc}}(X)$. 
Lemma 3.4.2. Let $(s, t): M \rightarrow X$ be a formal groupoid over $X$ satisfying the conditions of Theorem 3.3.1 (ii), and let $\mathcal{F} \in D_{\mathrm{qc}}(M)$ be a quasi-1-cocycle. Then $\mathcal{F} \otimes \omega_{s} \in D_{\mathrm{qct}}(M)$ has a natural structure of a convolution algebra over $M$.

Proof. As the convolution product on $\mathcal{F} \otimes \omega_{s}$ we take the map

$$
\begin{aligned}
m_{*}\left(p_{1}^{*} \mathcal{F} \otimes p_{2}^{*} \mathcal{F} \otimes p_{1}^{*} \omega_{s} \otimes p_{2}^{*} \omega_{s}\right) & \rightarrow m_{*}\left(m^{*} \mathcal{F} \otimes p_{1}^{*} \omega_{s} \otimes p_{2}^{*} \omega_{s}\right) \\
& \rightarrow \mathcal{F} \otimes m_{*}\left(p_{1}^{*} \omega_{s} \otimes p_{2}^{*} \omega_{s}\right) \rightarrow \mathcal{F} \otimes \omega_{s}
\end{aligned}
$$

on $M$, where we use consecutively the quasi-1-cocycle structure on $\mathcal{F}$, the projection formula, and the convolution product on $\omega_{s}$ constructed in Theorem 3.3.1. The associativity axiom follows from the associativity of the convolution product on $\omega_{S}$ and the compatibility on $M \times_{X} M \times_{X} M$ in the definition of quasi-1-cocycle. The unit on $\mathcal{F} \otimes \omega_{s}$ is given by the composition

$$
e_{*} \mathcal{O}_{X} \rightarrow \mathcal{F} \otimes e_{*} \mathcal{O}_{X} \rightarrow \mathcal{F} \otimes \omega_{s},
$$

where the maps are induced by the units for $\mathcal{F}$ and for $\omega_{s}$.

We are mostly interested in the case when $M$ is the action groupoid $\Gamma_{G}$ associated with an action of a group scheme (resp. formal group scheme) $G$ on $X$. In this case a 1-cocycle $\mathscr{L}$ of $G$ with values in $\operatorname{Pic}(X)$ can be viewed as a line bundle on $G \times X$, and an $\mathscr{L}$-twisted $G$-sheaf on $X$ is a quasi-coherent sheaf $F$ on $X$ equipped with an isomorphism

$$
F_{g x} \rightarrow \mathscr{L}_{g, x} \otimes F_{x}
$$

over $G \times X$, where $F_{g x}$ denotes the pull-back of $F$ under the map $G \times X \rightarrow$ $X:(g, x) \mapsto g x$, etc. This morphism should reduce to the identity over $e \times X$ (recall that $\mathscr{L}_{e, x}$ is trivialized), and the natural diagram on $G \times G \times X$ should be commutative. We denote the category of $\mathscr{L}$-twisted $G$-sheaves on $X$ by $\operatorname{Qcoh}_{G}^{\mathscr{L}}(X)$. In the case when $G$ is finite we also denote by $\operatorname{Coh}_{G}^{\mathscr{L}}(X) \subset \mathrm{Qcoh}_{G}^{\mathscr{L}}(X)$ the full subcategory consisting of coherent sheaves equipped with the above data. Note that for a line bundle $L$ on $X$ the coboundary $\delta L$ is given by $\delta L_{g, x}=L_{g x} \otimes L_{x}^{-1}$.

In the case when $G$ is a group scheme the category $\operatorname{Qcoh}_{G}^{\mathscr{L}}(X)$ should be viewed as a category twisted quasi-coherent sheaves on the global quotient stack $[X / G]$. The next lemma states the corresponding invariance of this category under certain changes of a presentation (and extends it to the case of formal group schemes).

Lemma 3.4.3. Assume that $G$ is finite group scheme (resp. locally nicely ind-finite formal group scheme) acting on $X$, and let $\left(X^{\prime}, G^{\prime}\right)$ be another data of the same kind. Assume that we have a surjective homomorphism of groups $G^{\prime} \rightarrow G$ with the kernel $K$ which is finite group scheme (flat over $S$ ), and a $G^{\prime}$-equivariant morphism $X^{\prime} \rightarrow X$. Assume also that $X^{\prime} \rightarrow X$ is a K-torsor. Let $\mathscr{L}$ be a 1-cocycle of $G$ with values in $\mathcal{P i c}(X)$, and let $\mathscr{L}^{\prime}$ be its pull-back to $G^{\prime} \times X^{\prime}$. Then $\operatorname{Qcoh}_{G}^{\mathscr{L}}(X)$ is equivalent to $\mathrm{Q} \operatorname{coh}_{G^{\prime}}^{\mathscr{L}^{\prime}}\left(X^{\prime}\right)$. 
The proof is a straightforward application of the flat descent for quasi-coherent sheaves.

With a 1-cocycle $\mathscr{L}$ of $G$ with values in $\operatorname{Pic}(X)$ one can associate a kernel representation of $G$ over $X$ by setting

$$
V_{\mathscr{L}}=\gamma_{G *}(\mathscr{L})
$$

where $\gamma_{G}: G \times X \rightarrow X \times G \times X:(g, x) \mapsto(g x, g, x)$. It is easy to see that for the trivial 1-cocycle we get precisely the kernel representation $V_{X}^{G}$ associated with the action of $G$ on $X$.

More generally, if $\mathscr{F}$ is a quasi-1-cocycle of $G$ with values in $D_{\mathrm{qc}}(X)$ then one we associate with $\mathscr{F}$ a kernel quasi-representation of $G$ over $X$ by

$$
V_{\mathscr{F}}:=\gamma_{G *}(\mathscr{F}) \in D_{\mathrm{qc}}(X \times G \times X) .
$$

This construction also works in the case when $G$ is a locally nicely ind-finite formal group scheme provided $\mathscr{F} \in D_{G, \vec{c}}(G \times X)$ (i.e., locally over $G$ the cohomology of $\mathcal{F}$ are in $A_{\overrightarrow{\mathrm{c}}}$ ). In this case we have the corresponding kernel algebra

$$
\mathcal{A}_{X}^{G}(\mathscr{F}):=\mathcal{A}\left(V_{\mathscr{F}}, \omega_{G / S}\right)=(g x, x)_{*}\left(p_{1}^{*} \omega_{G / S} \otimes \mathscr{F}\right)
$$

(see Proposition 3.2.4). One can easily check that this is the same kernel algebra as the one obtained from the convolution algebra structure on $\mathscr{F} \otimes p_{G}^{*} \omega_{G / S}$ constructed in Lemma 3.4.2, where $p_{G}: \Gamma_{G}=G \times X \rightarrow G$ is the projection. More precisely, one has the following statement (the proof is straightforward).

Lemma 3.4.4. Let $G$ be a finite group scheme (resp. locally nicely ind-finite formal group scheme) acting on $X$, and let $\mathcal{F} \in D_{\mathrm{qc}}(G \times X)\left(\right.$ resp. $\left.\mathcal{F} \in D_{G, \overrightarrow{\mathrm{c}}}(G \times X)\right)$ be a quasi-1-cocycle $\mathscr{F}$ of $G$ with values in $D_{\mathrm{qc}}(X)$. Then the push-forward of the convolution algebra $\mathscr{F} \otimes p_{G}^{*} \omega_{G / S}$ with respect to the homomorphism of groupoids $\gamma_{G}: \Gamma_{G} \rightarrow X \times G \times X$ can be identified with the convolution algebra $V_{\mathcal{F}} \otimes p_{2}^{*} \omega_{G / S}$ on $X \times G \times X$ associated with the quasi-representation $V_{\mathscr{F}}$ (see Proposition 3.2.4). Hence, we have an isomorphism of kernel algebras over $X$ :

$$
\mathcal{A}_{X}^{G}(\mathcal{F}) \simeq \mathcal{A}_{\Gamma_{G}}\left(\mathcal{F} \otimes p_{G}^{*} \omega_{G / S}\right) .
$$

We have the following analog of Corollary 3.3.2 for twisted $G$-equivariant sheaves that can be deduced from Theorem 3.4.1.

Corollary 3.4.5. Let $G$ be finite group scheme (resp. locally nicely ind-finite formal group scheme) acting on a scheme $X$, and let $\mathscr{L}$ be a 1-cocycle of $G$ with values in $\mathscr{P i c}(X)$. Then the category $\operatorname{Qcoh}_{G}^{\mathscr{L}}(X)$ is equivalent to $\mathcal{A}_{X}^{G}(\mathscr{L})$-mod. In the case when $G$ is finite, we also have $\operatorname{Coh}_{G}^{\mathscr{L}}(X) \simeq \mathcal{A}_{X}^{G}(\mathscr{L})-\bmod ^{\mathrm{c}}$. 
The dependences between the notions introduced above can be roughly summarized by the following picture:

Quasi 1-cocycles

Kernel quasi-representations $\stackrel{\text { Prop. 3.2.4 }}{\longrightarrow}$ Convolution algebras $\stackrel{\text { (3.2.2) }}{\longrightarrow}$ Kernel algebras

Quasi-1-cocycles appear naturally via the following construction.

Lemma 3.4.6. Let $G$ be a finite group scheme (resp. locally nicely ind-finite formal group scheme). Let $f: X \rightarrow Y$ be an affine $G$-equivariant morphism between $S$-schemes equipped with a $G$-action, and let $\mathscr{F}$ be a quasi-1-cocycle of $G$ with values in $D_{\mathrm{qc}}(X)$ (in the formal case we assume that $\mathscr{F} \in D_{G, \overrightarrow{\mathrm{c}}}(G \times X)$ ). Then $\left(\operatorname{id}_{G} \times f\right)_{*} \mathcal{F}$ has a natural structure of a quasi-1-cocycle of $G$ with values in $D_{\mathrm{qc}}(Y)$. Furthermore, we have an isomorphism of kernel algebras

$$
(f \times f)_{*} \mathcal{A}_{X}^{G}(\mathcal{F}) \simeq \mathcal{A}_{Y}^{G}\left(\left(\operatorname{id}_{G} \times f\right)_{*} \mathscr{F}\right) .
$$

Proof. Set $\mathcal{E}=\left(\operatorname{id}_{G} \times f\right)_{*} \mathcal{F}$. Note that in the formal case we have $\mathcal{E} \in D_{\mathrm{qc}}(G \times Y)$ by Proposition 1.2.2 (vii) since $f$ is an affine morphism. The unit $\mathcal{O}_{X} \rightarrow\left(e \times \mathrm{id}_{X}\right)^{*} \mathcal{F}$ for $\mathcal{F}$ induces a unit map

$$
\mathcal{O}_{X} \rightarrow\left(e \times \operatorname{id}_{Y}\right)^{*} \mathcal{E} \simeq f_{*}\left(e \times \operatorname{id}_{X}\right)^{*} \mathcal{F},
$$

where the latter isomorphism is given by the base change formula. Also, we have a natural morphism on $G \times G \times Y$ :

$$
\mathscr{G}_{g_{1}, g_{2} x} \otimes \mathcal{E}_{g_{2}, x} \rightarrow\left(\operatorname{id}_{G \times G} \times f\right)_{*}\left(\mathcal{F}_{g_{1}, g_{2} x} \otimes \mathcal{F}_{g_{2}, x}\right) .
$$

Hence, the quasi-1-cocycle structure on $\mathscr{F}$ induces a similar structure on $\mathscr{G}$.

Recall that $\mathcal{A}_{X}^{G}(\mathcal{F})$ comes from the convolution algebra $\mathcal{F} \otimes p_{G}^{*} \omega_{G / S}$ over the action groupoid $\Gamma_{G}(X)=G \times X$. Similarly, $\mathcal{A}_{Y}^{G}(\mathscr{E})$ comes from the convolution algebra $\mathscr{E} \otimes p_{G}^{*} \omega_{G / S}$ over $\Gamma_{G}(Y)=G \times Y$. The projection formula and the base change formula give an isomorphism

$$
\mathscr{G} \otimes p_{G}^{*} \omega_{G / S} \simeq\left(\operatorname{id}_{G} \times f\right)_{*}\left(\mathcal{F} \otimes p_{G}^{*} \omega_{G / S}\right)
$$

over $\Gamma_{G}(Y)$ which induces the required isomorphism (3.4.7). The compatibility of the product maps and of units is easy to check.

Proposition 3.4.7. Let $G$ be a finite group scheme (resp. locally nicely ind-finite formal group) acting on $X$, and let $\mathcal{F}$ be a flat quasi-coherent sheaf on $G \times X$ equipped with a quasi-1-cocycle structure. Then the kernel algebra $\mathcal{A}_{X}^{G}(\mathcal{F})$ is pure. If $G$ is a finite group scheme and $\mathcal{F}$ is a vector bundle then the pure kernel algebra $\mathcal{A}_{X}^{G}(\mathcal{F})$ is finite. 
Proof. By Lemma 3.4.4, we can realize $\mathcal{A}_{X}^{G}(\mathcal{F})$ as coming from the convolution algebra $\mathscr{F} \otimes p_{G}^{*} \omega_{G / S}$ on $\Gamma_{G}$. Now the first assertion follows easily from Lemma 3.2.3. The second assertion is clear since in this case $\mathcal{A}_{X}^{G}(\mathcal{F})$ is the push-forward of $\mathscr{F} \otimes$ $p_{G}^{*} \omega_{G / S}$ under a finite map.

Remark. It is easy to see that if we change a 1-cocycle $\mathscr{L}$ to a cohomologous cocycle $\mathscr{L} \otimes \delta L$ then the corresponding kernel representations of $G$ over $X$ are related via the autoequivalence of $D_{\mathrm{qc}}(X)$ given by tensoring with $L$ (as in Proposition 3.2.5):

$$
V_{\mathscr{L} \otimes \delta L} \simeq \Delta_{*} L \circ_{X} V_{\mathscr{L}} \circ_{X} \Delta_{*} L^{-1} .
$$

Hence, the corresponding kernel algebras are also related in a similar way.

Example. Assume that the action of $G$ on $X$ is trivial. Then a 1-cocycle of $G$ with values in $\operatorname{Pic}(X)$ is the same as a monoidal functor (over $S$ ) of stacks of groupoids $G \rightarrow \operatorname{Pic}(X)$ over $S$, i.e., in the terminology of [20], a central extension of $G$ by $\operatorname{Pic}(X))$. By definition, such a central extension is given by a line bundle $\mathscr{L}$ on $G \times X$ equipped with an isomorphism

$$
\mathscr{L}_{g_{1} g_{2}, x} \simeq \mathscr{L}_{g_{1}, x} \otimes \mathscr{L}_{g_{2}, x}
$$

satisfying the natural cocycle condition. In this case the morphism of groupoids $\Gamma_{G} \rightarrow X \times X$ is a composition of the natural projection $p_{2}: \Gamma_{G} \simeq G \times X \rightarrow X$ with the diagonal embedding $\Delta: X \rightarrow X \times X$. Hence,

$$
\mathcal{A}_{X}^{G}(\mathscr{L}) \simeq \Delta_{*} \mathcal{O}_{X}[G]^{\mathscr{L}},
$$

where

$$
\mathcal{O}_{X}[G]^{\mathscr{L}}:=p_{2 *}\left(p_{G}^{*} \omega_{G} \otimes \mathscr{L}\right)
$$

is an algebra in $D_{\mathrm{qc}}(X)$ associated with $\mathscr{L}$. Here the product on the right-hand side is induced by the convolution algebra structure on $p_{G}^{*} \omega_{G} \otimes \mathscr{L}$, where $G \times X$ is viewed as a group scheme (resp. formal group scheme) over $X$. Note that the algebra $\mathcal{O}_{X}[G]^{\mathscr{L}}$ is not always commutative even if $G$ is commutative. This corresponds to the fact that our monoidal functor $G \rightarrow \operatorname{Pic}(X)$ does not have to respect the commutativity constraints (i.e., it is not necessarily a homofunctor between the Picard categories). For example, if $X=S$ (the base scheme) then a 1-cocycle of $G$ with values in $\operatorname{Pic}(S)$ over $S$ is the same as a central extension of group schemes

$$
1 \rightarrow \mathbb{G}_{m} \rightarrow \widetilde{G} \rightarrow G \rightarrow 1 .
$$

The corresponding algebra is the twisted group algebra corresponding to this extension.

Now assume that $H$ is a group scheme (or a formal group scheme) acting on $X$ (and $G$ acts trivially on $X$ ). We say that an $H$-equivariant structure on a homofunctor 
$\mathscr{L}$ from $G$ to $\operatorname{Pic}(X)$ is given if $\mathscr{L}$ is equipped with an $H$-equivariant structure with respect to the natural action of $H$ on $G \times X$, and the isomorphism (3.4.8) is compatible with the $H$-action. In this situation we will also write that $\mathscr{L}$ gives a homofunctor $G \rightarrow \operatorname{Pic}^{H}(X)$. Recall that for a commutative group scheme $A$ we denote by $\operatorname{EXT}\left(A, \mathbb{G}_{m}\right)$ the Picard stack of (commutative) extensions of $A$ by $\mathbb{G}_{m}$. In the case when we have a homomorphism $H \rightarrow A$ we denote by $\operatorname{EXT}^{H}\left(A, \mathbb{G}_{m}\right)$ the Picard stack of extensions of $A$ by $\mathbb{G}_{m}$ equipped with a trivialization over $H$.

Lemma 3.4.8. (i) In the above situation the category of monoidal functors $G \rightarrow$ $\operatorname{Pic}^{H}(X)$ over $S$ is equivalent to the category of 1 -cocycles of $H \times G$ with values in $\operatorname{Pic}(X)$, trivialized over $H$.

(ii) Now assume that $X$ is a commutative group scheme and the action of $H$ on $X$ is given by translations (so we have a homomorphism $H \rightarrow X$ ). Then we can replace Pic $(X)$ and $\mathcal{P i c}^{H}(X)$ by the Picard stacks $\operatorname{EXT}\left(X, \mathbb{G}_{m}\right)$ and $\operatorname{EXT}^{H}\left(X, \mathbb{G}_{m}\right)$, respectively. Assume also that $G$ is a commutative group scheme or a formal group scheme. Then the following two categories are equivalent:

(a) biextensions of $G \times X$ by $\mathbb{G}_{m}$ trivialized over $G \times H$;

(b) homofunctors $G \rightarrow \operatorname{EXT}^{H}\left(X, \mathbb{G}_{m}\right)$.

This category is also equivalent to a full subcategory in the category of 1-cocycles of $H \times G$ with values in $\operatorname{EXT}\left(X, \mathbb{G}_{m}\right)$, trivialized over $H$.

Proof. (i) To a monoidal functor $\mathscr{L}: G \rightarrow \mathcal{P i c}^{H}(X)$ over $S$ we associate the line bundle $p_{23}^{*} \mathscr{L}$ on $H \times G \times X$. It is easy to see that it is equipped with the required structures. The inverse functor is given by the restriction to $e_{H} \times G \times X$, where $e_{H} \in H$ is the unit.

(ii) The equivalence of (a) and (b) follows from Proposition 1.3.3. To realize the category in (b) in terms of 1-cocycles of $H \times G$, trivialized over $H$, use (i).

3.5. 1-cocycles and Cartier duality. Henceforward, whenever formal $k$-groups are mentioned it is assumed that $S=\operatorname{Spec}(k)$, where $k$ is a field of characteristic zero.

Let $X$ be an $S$-scheme, $G$ a finite commutative group scheme (flat) over $S$ (resp. formal $k$-group), and let $G^{*}$ denote the Cartier dual group scheme (resp. affine commutative algebraic group over $k$ ).

Given a $G^{*}$-torsor $\phi: E \rightarrow X$ consider its pull-back to $G \times X:\left(\operatorname{id}_{G} \times \phi\right): G \times$ $E \rightarrow G \times X$. Viewing the universal bicharacter $b: G \times G^{*} \rightarrow \mathbb{G}_{m}$ as a homomorphism of group schemes $G^{*} \rightarrow \mathbb{G}_{m}$ over $G \times X$ we can associate with the above $G^{*}$-torsor a line bundle $\mathscr{L}_{E}$ over $G \times X$. It is easy to see that $\mathscr{L}_{E}$ has a natural structure of a homofunctor $G \rightarrow \operatorname{Pic}(X)$. Note that this construction is local over $X$. Hence, the obtained homofunctor is locally trivial, i.e., there exists an open covering $\mathfrak{U} \rightarrow X$ such that the induced homofunctor $G \rightarrow \mathcal{P i c}(\mathfrak{U})$ is trivial.

Proposition 3.5.1. (i) The above construction gives an equivalences between the category of $G^{*}$-torsors over $X$ and the category of locally trivial homofunctors 
$G \rightarrow \operatorname{Pic}(X)$. Furthermore, for $a G^{*}$-torsor $\phi: E \rightarrow X$ we have an isomorphism of sheaves of $\mathcal{O}_{X}$-algebras

$$
\phi_{*} \mathcal{O}_{E} \simeq \mathcal{O}_{X}[G]^{\mathscr{L}_{E}},
$$

where the algebra structure on the right is defined by (3.4.9).

(ii) Assume now that $X$ is a formal scheme over $k$ and $G$ is a formal $k$-group. As above, we have an equivalence between the category of $G$-torsors over $X$ and the category of locally trivial homofunctors $G^{*} \rightarrow \operatorname{Pic}(X)$. For a $G$-torsor $\psi: E \rightarrow X$ and a torsion quasi-coherent sheaf $F$ on $X$ we have a natural isomorphism

$$
\psi_{!} \psi^{!} F \simeq F \otimes p_{2 *} \mathscr{L}_{E},
$$

of sheaves on $X$, where $\mathscr{L}_{E}$ is a line bundle on $G^{*} \times X$ associated with $E$.

Proof. (i) The quasi-inverse functor is constructed as follows. Given a locally trivial homofunctor $\mathscr{L}: G \rightarrow \operatorname{Pic}(X)$, consider the functor of trivializations of $\mathscr{L}$ on the category of $X$-schemes. By definition, it associates with an $X$-scheme $S$ the set of isomorphisms of the pull-back of $\mathscr{L}$ to $G \rightarrow \operatorname{Pic}(S)$ with the trivial homofunctor. It is easy to see that this functor is represented by a $G^{*}$-torsor.

To construct an isomorphism of $\mathcal{O}_{X}$-algebras we consider their pull-backs to $E$. For the left-hand side we have a $G$-equivariant isomorphism of algebras

$$
\phi^{*} \phi_{*} \mathcal{O}_{E} \simeq \pi_{E}^{*}\left(\pi_{G^{*}}\right)_{*} \mathcal{O}_{G^{*}},
$$

where for every $S$ scheme $Y$ we denote by $\pi_{Y}: Y \rightarrow S$ the projection to $S$. On the other hand, the canonical trivialization of $\phi^{*} \mathscr{L}_{E}$ gives rise to an algebra isomorphism

$$
\phi^{*} \mathcal{O}_{X}[G]^{\mathscr{L}_{E}} \simeq \pi_{E}^{*}\left(\pi_{G}\right)_{*} \omega_{G},
$$

where $\left(\pi_{G}\right)_{*} \omega_{G}$ is equipped with the convolution product. It remains to use the $G^{*}$-equivariant isomorphism

$$
\left(\pi_{G^{*}}\right)_{*} \mathcal{O}_{G^{*}} \simeq\left(\pi_{G}\right)_{!} \omega_{G} .
$$

(ii) It is enough to construct such an isomorphism for the trivial $G$-torsor and to check its functoriality with respect to automorphisms of the trivial $G$-torsor. In this case we have $E=G \times X$ and $\mathscr{L}_{E}$ is trivial; $\psi: G \times X \rightarrow X$ is the projection. Hence, we have to construct an isomorphism

$$
\psi ! \psi^{!} F \simeq F \otimes \pi_{X}^{*}\left(\pi_{G^{*}}\right)_{*} \mathcal{O}_{G^{*}},
$$

compatible with automorphisms of the trivial $G$-torsor over $X$. Note that the projection $\psi: G \times X \rightarrow X$ has a nicely ind-finite structure (since $G$ is a union of finite $k$-schemes). Hence, by Proposition 1.2.5 (iii), we have $\psi^{!} F \simeq \psi^{*} F \otimes \psi^{!} \mathcal{O}_{X}$. By the projection formula, this leads to an isomorphism

$$
\psi_{!} \psi^{!} F \simeq F \otimes \psi_{!} \psi^{!} \mathcal{O}_{X} .
$$


Using the compatibility of the formation of $f^{!} \mathcal{O}_{X}$ with base changes (see Proposition 1.2.5 (iii)) and the base change formula (Theorem 1.2.4 (ii), (iii)) we get

$$
F \otimes \psi ! \psi^{!} \mathcal{O}_{X} \simeq F \otimes \psi ! p_{G}^{*} \omega_{G} \simeq F \otimes \pi_{X}^{*}\left(\pi_{G}\right) ! \omega_{G},
$$

where $p_{G}: G \times X \rightarrow G$ is the projection. It remains to use the $G$-equivariant isomorphism (3.5.1). The compatibility of (3.5.2) with automorphisms of both sides induced by automorphisms of the trivial $G$-torsor over $X$ follows easily from the compatibility of the isomorphism (3.5.1) with the natural $G$-action on both sides.

It follows that in the situation of Proposition 3.5.1 (ii) we have an isomorphism

$$
\Delta_{*} \phi_{*} \mathcal{O}_{E} \simeq \mathcal{A}_{X}^{G}\left(\mathscr{L}_{E}\right)
$$

of kernel algebras over $X$.

Now assume that $H$ is a group scheme (resp. formal $k$-group) acting on $X$. Since the equivalence of the above proposition is compatible with the pull-back functors (with respect to morphisms $X^{\prime} \rightarrow X$ ), we deduce the following corollary.

Corollary 3.5.2. Let $\phi: E \rightarrow X$ be a $G^{*}$-torsor and let $\mathscr{L}_{E}$ be the corresponding homofunctor $G \rightarrow \operatorname{Pic}(X)$. A lifting of the $H$-action on $X$ to an action on $E$ (commuting with $G^{*}$ ) is the same as an $H$-equivariant structure on $\mathscr{L}_{E}$ (compatible with the homofunctor structure).

Now let us consider a special case when $X$ is a commutative group scheme (resp. formal $k$-group). Then instead of considering all $G^{*}$-torsors we can restrict our attention to extensions $E$ of $X$ by $G^{*}$ in the category of commutative groups. Here is the corresponding specialization of Proposition 3.5.1.

Lemma 3.5.3. (i) The construction of Proposition 3.5.1 induces an equivalence between the category of extensions of $X$ by $G^{*}$ (resp. $G$ ) in the category of sheaves of commutative groups and the category of biextensions of $G \times X\left(\right.$ resp. $\left.G^{*} \times X\right)$ by $\mathbb{G}_{m}$.

(ii) Let $H \rightarrow X$ be a homomorphism. Then the category of Lemma 3.4.8 (ii) is equivalent to the category of extensions $E$ of $X$ by $G^{*}$ in the category of sheaves of commutative groups, equipped with a splitting $H \rightarrow E$ over $H$.

Proof. (i) This follows from Proposition 1.3.4 because of the vanishings

$$
\underline{\operatorname{Ext}}^{1}\left(G, \mathbb{G}_{m}\right)=\underline{\operatorname{Ext}}^{1}\left(G^{*}, \mathbb{G}_{m}\right)=0
$$

(see Lemma 1.3.5; in the case when $G$ is a formal $k$-group we use Lemma 1.3.6(i)).

(ii) This follows immediately from part (i) using the definition (a) of Lemma 3.4.8 (ii). 
Now let us go back to the situation when $X$ does not have a group structure. Assume that $H$ is a finite commutative group scheme (resp. formal $k$-group) acting on $X, G$ is a finite commutative group scheme (resp. formal $k$-group), and $\phi: E \rightarrow X$ is a $G^{*}$-torsor equipped with an $H$-action that commutes with the $G^{*}$-action and is compatible with the $H$-action on $X$. In addition, assume that we have an extension of commutative group schemes (resp. formal $k$-groups)

$$
0 \rightarrow G \rightarrow \widetilde{H} \rightarrow H \rightarrow 0 .
$$

Let $\mathscr{B}$ be the corresponding biextension of $H \times G^{*}$ by $\mathbb{G}_{m}$ (see Lemma 3.5.3 (i)). We would like to identify the kernel algebra $(\phi \times \phi)_{*} \mathcal{H}_{E}^{H}(\mathcal{M})$, where $\mathcal{M}$ is a 1-cocycle of $H$ with values in $\mathcal{P i c}(E)$, with some kernel algebra corresponding to the action of $\widetilde{H}$ on $X$ (and a 1-cocycle). For this we need to impose the following compatibility of $\mathcal{M}$ with the biextension $\mathscr{B}$.

Definition. Let $\mathcal{M}$ be a 1-cocycle of $H$ with values in $\operatorname{Pic}(E)$. We say that $\mathcal{M}$ is equipped with $\left(G^{*}, \mathscr{B}\right)$-equivariant structure if an isomorphism

$$
\mathcal{M}_{h, g^{*} e} \simeq \mathcal{B}_{h, g^{*}} \otimes \mathcal{M}_{h, e}
$$

is given, and the following diagrams on $H \times H \times G^{*} \times E$ and $H \times G^{*} \times G^{*} \times E$ are commutative:

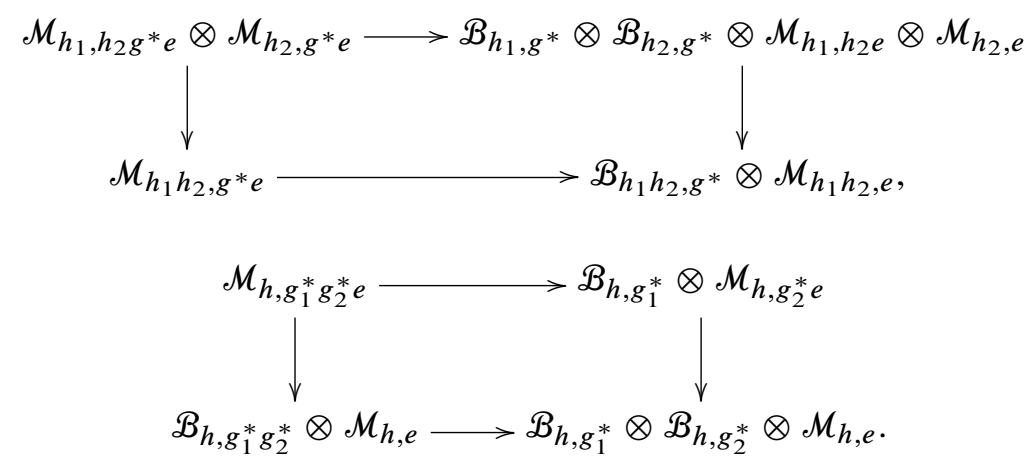

Since the pull-back of $\mathscr{B}$ to $\widetilde{H} \times G^{*}$ is trivial, the pull-back of a $\left(G^{*}, \mathscr{B}\right)$ equivariant 1 -cocycle $\mathcal{M}$ of $H$ with values in $\mathcal{P i c}(E)$ to $\widetilde{H} \times E$ is equipped with a $G^{*}$-equivariant structure. Hence, it descends to a 1-cocycle $\widetilde{\mathcal{M}}$ of $\widetilde{H}$ with values in $\operatorname{Pic}(X)$. It is easy to see that the restriction of $\widetilde{\mathcal{M}}$ to $G$ is naturally isomorphic to $\mathscr{L}_{E}: G \rightarrow \operatorname{Pic}(X)$, the homofunctor associated with $E$.

Proposition 3.5.4. The above construction $\mathcal{M} \mapsto \tilde{\mathcal{M}}$ gives an equivalences between the categories of $\left(G^{*}, \mathscr{B}\right)$-equivariant 1 -cocycles $\mathcal{M}$ of $H$ with values in $\mathcal{P i c}(E)$ and 1 -cocycles $\widetilde{\mathcal{M}}$ of $\widetilde{H}$ with values in $\operatorname{Pic}(X)$ extending $\mathscr{L}_{E}$. Furthermore, we have an isomorphism of convolution algebras

$$
p_{1}^{*} \omega_{H} \otimes\left(\operatorname{id}_{H} \times \phi\right)_{*} \mathcal{M} \simeq\left(\pi \times \operatorname{id}_{X}\right) !\left(p_{1}^{*} \omega_{\tilde{H}} \otimes \tilde{\mathcal{M}}\right)
$$


over $H \times X$ (viewed as a groupoid over $X$ ), where $\pi: \widetilde{H} \rightarrow H$ is the projection. Here the left-hand side is the convolution algebra associated with the quasi-1-cocycle structure on $\left(\mathrm{id}_{H} \times \phi\right)_{*} \mathcal{M}$ (see Lemma 3.4.6), and the right-hand side is the pushforward of the convolution algebra associated with $\widetilde{\mathcal{M}}$ with respect to the map $\pi \times$ id: $\widetilde{H} \times X \rightarrow H \times X$ of groupoids over $X$ (see Lemma 3.2.2). Hence, we have an isomorphism

$$
(\phi \times \phi)_{*} \mathcal{A}_{E}^{H}(\mathcal{M}) \simeq \mathcal{A}_{X}^{\widetilde{H}}(\tilde{\mathcal{M}})
$$

of kernel algebras over $X$

Proof. Let us show how to go back from a 1-cocycle $\tilde{\mathcal{M}}$ of $\tilde{H}$ with values in $\operatorname{Pic}(X)$ extending $\mathscr{L}_{E}$ to a $\left(G^{*}, \mathscr{B}\right)$-equivariant 1-cocycle of $H$ with values in $\operatorname{Pic}(E)$. Let $\mathcal{M}^{\prime}$ denote the pull-back of $\widetilde{\mathcal{M}}$ under the map id $\times \phi: \widetilde{H} \times E \rightarrow \widetilde{H} \times X$. Then $\mathcal{M}^{\prime}$ is a 1-cocycle of $\widetilde{H}$ with values in $\operatorname{Pic}(X)$. Note that the pull-back of $\mathscr{L}_{E}$ under the map $\operatorname{id}_{G} \times \phi: G \times E \rightarrow G \times X$ is naturally trivialized, and the $G^{*}$-equivariant structure on it (where $G^{*}$ acts on $E$ ) is induced by the universal bicharacter $G \times G^{*} \rightarrow \mathbb{G}_{m}$. It follows that $\mathcal{M}^{\prime}$ restricts to the trivial 1-cocycle of $G$, so it descends to a 1-cocycle $\mathcal{M}$ of $\widetilde{H} / G \simeq H$ with values in $\operatorname{Pic}(E)$. The $\left(G^{*}, \mathscr{B}\right)$-equivariant structure on $\mathcal{M}$ comes from the $G^{*}$-equivariance of $\mathcal{M}^{\prime}$ (recall that the pull-back of $\mathscr{B}$ to $\widetilde{H} \times G^{*}$ is naturally trivialized but $G$ acts on it via the universal bicharacter $G \times G^{*} \rightarrow \mathbb{G}_{m}$ ).

To construct an isomorphism (3.5.4) let us first consider the pull-backs of both sides under the map $\operatorname{id}_{H} \times \phi: H \times E \rightarrow H \times X$. By the definition, we have an isomorphism

$$
\left(\pi \times \operatorname{id}_{E}\right)^{*} \mathcal{M} \simeq\left(\operatorname{id}_{\widetilde{H}} \times \phi\right)^{*} \cdot \tilde{\mathcal{M}}
$$

over $\widetilde{H} \times E$, compatible with 1-cocycle structures (for $\widetilde{H}$ ). Applying the flat base change formula for the cartesian diagram

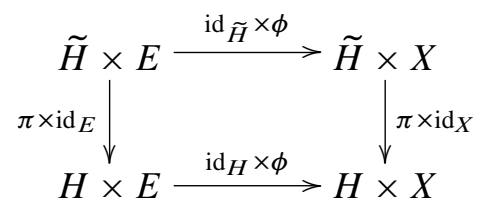

and the projection formula for the morphism $\pi \times \mathrm{id}_{E}$ we derive an isomorphism

$$
\left(\operatorname{id}_{H} \times \phi\right)^{*}\left(\pi \times \operatorname{id}_{X}\right) !\left(p_{1}^{*} \omega_{\tilde{H}} \otimes \tilde{\mathcal{M}}\right) \simeq\left(\pi \times \operatorname{id}_{E}\right)_{!}\left(p_{1}^{*} \omega_{\widetilde{H}}\right) \otimes \mathcal{M}
$$

of convolution algebras over $H \times E$ (viewed as a groupoid over $E$ ). Another use of the base change formula gives an isomorphism of this with $p_{1}^{*}\left(\pi ! \omega_{\tilde{H}}\right) \otimes \mathcal{M}$. Note that we can view $\pi: \widetilde{H} \rightarrow H$ as a $G$-torsor over $H$ (see Lemma 1.3.6 (ii)). Hence, using the isomorphism of Proposition 3.5.1 (ii) we get

$$
\pi ! \omega_{\widetilde{H}} \simeq \pi ! \pi^{!} \omega_{H} \simeq \omega_{H} \otimes p_{*} \mathcal{B} .
$$


Thus, we derive an isomorphism

$$
\left(\operatorname{id}_{H} \times \phi\right)^{*}\left(\pi \times \operatorname{id}_{X}\right) !\left(p_{1}^{*} \omega_{\tilde{H}} \otimes \tilde{\mathcal{M}}\right) \simeq p_{1}^{*}\left(\omega_{H} \otimes p_{*} \mathcal{B}\right) \otimes \mathcal{M}
$$

On the other hand, using the $\left(G_{*}, \mathscr{B}\right)$-equivariance of $\mathcal{M}$ we get

$$
\left(\operatorname{id}_{H} \times \phi\right)^{*}\left(\operatorname{id}_{H} \times \phi\right)_{*} \mathcal{M} \simeq p_{13 *}\left(p_{12}^{*} \mathscr{B}\right) \otimes \mathcal{M} \simeq p_{1}^{*}\left(p_{*} \mathscr{B}\right) \otimes \mathcal{M},
$$

where $p_{i j}$ are projections from $H \times G^{*} \times E, p_{1}: H \times E \rightarrow H$ and $p: H \times G^{*} \rightarrow H$ are also projections. Hence,

$$
\left(\operatorname{id}_{H} \times \phi\right)^{*}\left(p_{1}^{*} \omega_{H} \otimes\left(\operatorname{id}_{H} \times \phi\right)_{*} \mathcal{M}\right) \simeq p_{1}^{*}\left(\omega_{H} \otimes p_{*} \mathscr{B}\right) \otimes \mathcal{M}
$$

Comparing this with (3.5.6) we get an isomorphism of the pull-backs of both sides of (3.5.4) under $\operatorname{id}_{H} \times \phi$, compatible with convolution algebra structures. It is easy to check that it is also compatible with $G^{*}$-actions, so it descends to an isomorphism over $H \times X$.

The isomorphism (3.5.5) follows from (3.5.4) by passing to associated kernel algebras, taking into account the isomorphism

$$
(\phi \times \phi)_{*} \mathcal{A}_{E}^{H}(\mathcal{M}) \simeq \mathcal{A}_{X}^{H}\left((\mathrm{id} \times \phi)_{*} \mathcal{M}\right)
$$

that follows from Lemma 3.4.6.

By Lemma 3.4.8(i), an $H$-equivariant structure on a homofunctor $\mathscr{L}: G \rightarrow$ $\operatorname{Pic}(X)$ gives rise to an extension of $\mathscr{L}$ to a 1 -cocycle $\widetilde{\mathscr{L}}$ of $H \times G$ with values in $\operatorname{Pic}(X)$, trivial over $H$. Hence, we have the corresponding pure kernel algebra $\mathcal{A}_{X}^{H} \times G(\widetilde{\mathscr{L}})$ over $X$.

On the other hand, a lifting of the $H$-action on $X$ to an action on $E$ gives rise to a pure kernel algebra $\mathcal{A}_{E}^{H}$ over $E$. Hence, we get a pure kernel algebra $(\phi \times \phi)_{*} \mathcal{A}_{E}^{H}$ over $X$.

Corollary 3.5.5. Assume that $G$ and $H$ are finite commutative group schemes (resp. formal $k$-groups), $X$ is a scheme with $H$-action, $\phi: E \rightarrow X$ is a $G^{*}$-torsor equipped with an $H$-action, $\mathscr{L}_{E}: G \rightarrow \mathcal{P i c}(X)$ is the corresponding $H$-equivariant homofunctor, and $\widetilde{\mathscr{L}}_{E}$ is the corresponding 1-cocycle of $H \times G$ with values in $\operatorname{Pic}(X)$. Then there is an isomorphism of derived kernel algebras over $X$ :

$$
(\phi \times \phi)_{*} \mathcal{A}_{E}^{H} \simeq \mathcal{A}_{X}^{H \times G}\left(\tilde{\mathscr{L}}_{E}\right) .
$$

3.6. Compatibility with open coverings. We are going to give a criterion for a kernel algebra coming from a convolution algebra to be of affine type (see Section 2.3).

Definition. Let $X$ be a scheme, and let $M$ be a formal groupoid over $X$. We say that $M$ is compatible with an open covering $\mathfrak{U}=\bigsqcup_{i} U_{i} \rightarrow X$ (in flat topology) if an isomorphism $\alpha: M_{\mathfrak{U} \times X} \stackrel{\sim}{\longrightarrow} M_{X \times \mathfrak{U}}$ over $M$ is given, where for a scheme $Y$ over 
$X \times X$ we denote by $M_{Y}$ the fibered product $M \times_{X \times X} Y$. We require $\alpha$ to satisfy the following compatibilities. First, the diagram

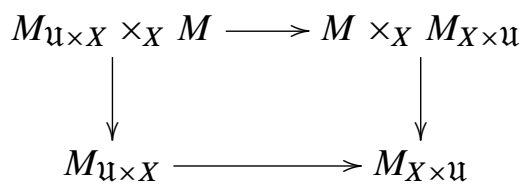

should be commutative, where the vertical arrows are obtained from the product map $m: M \times_{X} M \rightarrow M$ by the base change, and the upper arrow is given by the composition

$$
\begin{aligned}
M_{\mathfrak{U} \times X} \times_{X} M \simeq\left(M \times_{X} M\right)_{\mathfrak{U} \times X \times X} & \stackrel{\alpha_{1}}{\longrightarrow}\left(M \times_{X} M\right)_{X \times \mathfrak{U} \times X} \\
& \stackrel{\alpha_{2}}{\longrightarrow}\left(M \times_{X} M\right)_{X \times X \times \mathfrak{U}} \simeq M \times_{X} M_{X \times \mathfrak{U}},
\end{aligned}
$$

with $\alpha_{1}$ and $\alpha_{2}$ induced by $\alpha$. Second, the composition of $\alpha$ with the map $u_{1}: \mathfrak{U} \rightarrow$ $M_{\mathfrak{U} \times X}$ should coincide with the map $u_{2}: \mathfrak{U} \rightarrow M_{X \times \mathfrak{U}}$, where $u_{1}$ and $u_{2}$ obtained by the base change from the unit map $u: X \rightarrow M$.

Proposition 3.6.1. Let $M$ be a groupoid over $X$ (resp. formal groupoid over $X$ such that the structure maps $s, t: M \rightarrow X$ are flat and of ldu-pseudofinite type). Assume that $M$ is compatible with an open covering $j: \mathfrak{U}=\bigsqcup_{i} U_{i} \rightarrow X$, where $j_{i}: U_{i} \rightarrow X$ are affine morphisms. Then for every convolution algebra $\mathcal{V}$ on $M$ the kernel algebra $\mathscr{A}_{M}(\mathcal{V})$ over $X$ is compatible with this open covering.

Proof. By definition, $\mathcal{A}_{M}(\mathcal{V})$ is the push-forward of $\mathcal{V}$ with respect to the structure morphism $\pi: M \rightarrow X \times X$. Let us denote by $j_{1}: M_{\mathfrak{U} \times X} \rightarrow M$ and $j_{2}: M_{X \times \mathfrak{U}} \rightarrow M$ the natural morphisms. Then

$$
\mathcal{A}_{M}(\mathcal{V}){ }^{\circ} X(\mathfrak{H}) \simeq(\mathrm{id} \times j)_{*}(\mathrm{id} \times j)^{*} \mathcal{A}_{M}(\mathcal{V})
$$

can be identified with the push-forward to $X \times X$ of $j_{2 *} j_{2}^{*} \mathcal{V} \in D_{\mathrm{qc}}(M)$. Similarly, $\Delta(\mathfrak{U}){ }^{\circ} X \mathcal{A}_{M}(\mathcal{V})$ can be identified with the push-forward of $j_{1 *} j_{1}^{*} \mathcal{V} \in D_{\mathrm{qc}}(M)$. Since we have an isomorphism of $M_{X \times \mathfrak{U}}$ and $M_{\mathfrak{U} \times X}$ over $M$, this leads to the required isomorphism

$$
\mathcal{A}_{M}(\mathcal{V}) \circ_{X} \Delta(\mathfrak{U}) \simeq \Delta(\mathfrak{U}) \circ_{X} \mathcal{A}_{M}(\mathcal{V}) .
$$

Compatibility with the product (resp. unit) on $\mathcal{A}_{M}(\mathcal{V})$ follows from the commutativity of the diagram (resp. compatibility of $\alpha$ with $u_{1}$ and $u_{2}$ ) in the above definition.

For a formal group scheme $G$, ldu-pf (ldu-pseudofinite) over a field $k$, we denote by $G_{0}$ its connected component of 1 so that $G_{0}$ is infinitesimal, and $G / G_{0}$ is an étale formal group corresponding to the $\mathrm{Gal}(\bar{k} / k)$-module $G(\bar{k})$. 
Proposition 3.6.2. Let $G$ be a finite group scheme acting on $X$. Then for every flat quasi-coherent sheaf $\mathcal{F}$ on $G \times X$ equipped with a quasi-1-cocycle structure, the kernel algebra $\mathcal{A}_{X}^{G}(\mathcal{F})$ is pure and of affine type. Similar result holds if $S=\operatorname{Spec}(k)$, where $k$ is a field and $G$ is a formal group scheme, ldu-pf over $k$, such that the action of $\mathrm{Gal}(\bar{k} / k)$ on $G(\bar{k})$ factors through $\mathrm{Gal}\left(k^{\prime} / k\right)$ for some finite field extension $k \subset k^{\prime}$.

Proof. Recall that $\mathcal{A}_{X}^{G}(\mathcal{F})$ is the kernel algebra associated with a convolution algebra structure on the sheaf $\mathscr{F} \otimes p_{G}^{*} \omega_{G / S}$ over the action groupoid $\Gamma_{G}$ (see Lemma 3.4.4). It is pure by Proposition 3.4.7. To check that it is of affine type, by Proposition 3.6.1, it suffices to find an open affine covering $\mathfrak{U} \rightarrow X$ such that the action groupoid $\Gamma_{G}$ is compatible with $\mathfrak{U}$.

Consider first the case when $G$ is a finite group scheme over $S$ (recall that we assume it to be flat over $S$ ). We can assume that $S$ is affine and pick a finite open affine covering $\widetilde{\mathcal{U}} \rightarrow X$. Then setting $\mathfrak{U}=G \times \widetilde{\mathcal{U}}$ we obtain a $G$-equivariant open affine covering of $X$ in flat topology, where the map $\mathcal{U} \rightarrow X$ is the composition

$$
\mathfrak{U}=G \times \tilde{\mathfrak{U}} \rightarrow G \times X \rightarrow X
$$

(the last arrow is given by the $G$-action on $X$ ).

Now let us consider the case when $S=\operatorname{Spec}(k)$ and $G$ is a formal group scheme, $l d u$-pf over $k$. We start by choosing an arbitrary Zariski open affine covering $\widetilde{\mathcal{U}}=$ $\left(\widetilde{U}_{i}\right)$ of $X$. Since the action of $G_{0}$ preserves each open subset $\widetilde{U}_{i} \subset X$, for every $g \in G(\bar{k})$ there is a well-defined affine open subset $g\left(\widetilde{U}_{i}\right) \subset X_{\bar{k}}$, where $X_{\bar{k}}$ is obtained from $X$ by extending scalars to $\bar{k}$. If $O \subset G(\bar{k})$ is a $\operatorname{Gal}(\bar{k} / k)$-orbit (necessarily finite by our assumption) then the affine scheme $\bigsqcup_{g \in O} g\left(\widetilde{U}_{i}\right)$ comes from an étale open $U_{O, i} \rightarrow X$. These form an étale covering of $X$. Furthermore, it is easy to see that there is an action of $G$ on $\left(U_{O, i}\right)$ compatible with its action on $X$, as required.

3.7. Projective kernel representations and the Fourier-Mukai transform. The formalism of the Fourier-Mukai transform for abelian schemes requires to introduce a projective version of the notion of kernel representation (similar to the notion of projective representation of a group).

Definition. (i) Let $G$ be a group scheme (resp. formal group scheme) over $S$. A 2-cocycle of $G$ with values in $\operatorname{Pic}(S)$ is a line bundle $\mathscr{L}$ over $G \times G$ equipped with an isomorphism

$$
\mathscr{L}_{g_{1}, g_{2}} \otimes \mathscr{L}_{g_{1} g_{2}, g_{3}} \stackrel{\sim}{\longrightarrow} \mathscr{L}_{g_{1}, g_{2} g_{3}} \otimes \mathscr{L}_{g_{2}, g_{3}}
$$


on $G^{3}$ such that the following diagram on $G^{4}$ is commutative:

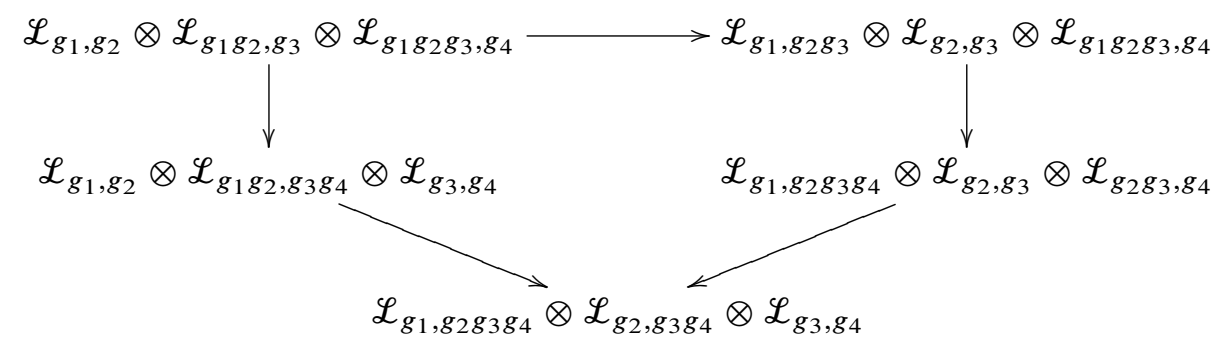

In addition, we assume that the bundle $(e \times e)^{*} \mathscr{L}=\mathscr{L}_{e, e}$ on $S$ is trivialized, where $e: S \rightarrow G$ is a neutral element. This induces a trivialization of the bundles $\mathscr{L}_{e, g}$ and $\mathscr{L}_{g, e}$ on $G$ (using the cocycle isomorphism).

(ii) Given a 2-cocycle $\mathscr{L}$ of a group scheme $G$ with values in $\operatorname{Pic}(S)$ and a scheme $X$ over $S$, a projective kernel representation of $G$ with the cocycle $\mathscr{L}$ over $X$ is an object $V \in D_{\mathrm{qc}}(X \times G \times X)$ equipped with an isomorphism

$$
\mu: V \circ_{X} V \stackrel{\sim}{\longrightarrow}\left(\operatorname{id}_{X} \times m \times \operatorname{id}_{X}\right)^{*} V \otimes p_{23}^{*} \mathscr{L}
$$

over $X \times G \times G \times X$ (where $m: G \times G \rightarrow G$ is the product), and an isomorphism

$$
u: \Delta_{*} \mathcal{O}_{X} \stackrel{\sim}{\longrightarrow}\left(\mathrm{id}_{X} \times e \times \mathrm{id}_{X}\right)^{*} V
$$

subject to the following conditions. The morphism $\mu$ induces the following morphism on $X \times G^{3} \times X$ :

$$
\begin{aligned}
\left(V \circ_{X} V\right){ }_{X} V & \rightarrow\left(\left(\operatorname{id}_{X} \times m \times \mathrm{id}_{X}\right)^{*} V \otimes p_{23}^{*} \mathscr{L}\right){ }_{X} V \\
& \simeq \mathscr{L}_{g_{1}, g_{2}} \otimes\left(\operatorname{id}_{X} \times m \times \operatorname{id}_{G} \times \operatorname{id}_{X}\right)^{*}\left(V \circ_{X} V\right) .
\end{aligned}
$$

Using $\mu$ again we get a map (an isomorphism)

$$
\left(V \circ_{X} V\right){ }_{X} V \rightarrow \mathscr{L}_{g_{1}, g_{2}} \otimes \mathscr{L}_{g_{1} g_{2}, g_{3}} \otimes\left(\operatorname{id}_{X} \times m m \times \mathrm{id}_{X}\right)^{*} V,
$$

where $m m: G^{3} \rightarrow G$ sends $\left(g_{1}, g_{2}, g_{3}\right)$ to $g_{1} g_{2} g_{3}$. Similarly, we construct a map

$$
V \circ_{X}\left(V \circ_{X} V\right) \rightarrow \mathscr{L}_{g_{1}, g_{2} g_{3}} \otimes \mathscr{L}_{g_{2}, g_{3}} \otimes\left(\mathrm{id}_{X} \times m m \times \mathrm{id}_{X}\right)^{*} V,
$$

and we require these maps to be the same. The maps $u$ and $\mu$ should be compatible as follows: the composition

$$
V \simeq \Delta_{*} \mathcal{O}_{X} \circ_{X} V \stackrel{u^{\circ} X}{\rightarrow}\left(\operatorname{id}_{X} \times e \times \operatorname{id}_{X}\right)^{*} V{ }^{\circ} V \rightarrow V
$$

where the second arrow is induced by $\left(\operatorname{id}_{X} \times e \times \operatorname{id}_{G} \times \mathrm{id}_{X}\right)^{*} \mu$ and by the trivialization of $\left(e \times \mathrm{id}_{X}\right)^{*} \mathscr{L}$ (resp. similar composition starting with id ${ }^{\circ} X u$ ) should be equal to the identity map. 
In the case when $G$ is a formal group scheme the above definition still make sense provided $V \in D_{G, \overrightarrow{\mathrm{c}}}(X \times G \times X)$ and the support of $V$ is proper over $X \times G$ and over $G \times X$.

(iii) Given a 2-cocycle $\mathscr{L}$ of $G$ as above and an $S$-scheme $X$ equipped with an action of $G$, a coboundary for $\mathscr{L}$ with values in $\operatorname{Pic}(X)$ is a line bundle $\mathcal{M}$ over $G \times X$ together with an isomorphism

$$
\alpha\left(g_{1}, g_{2} ; x\right): \mathcal{M}_{g_{1}, g_{2} x} \otimes \mathcal{M}_{g_{2}, x} \stackrel{\sim}{\longrightarrow} \mathcal{M}_{g_{1} g_{2}, x} \otimes \mathscr{L}_{g_{1}, g_{2}}
$$

on $G \times G \times X$ and a trivialization of $\left(e \times \mathrm{id}_{X}\right)^{*} \mathcal{M}=\mathcal{M}_{e, x}$ compatible with $\alpha(e, e ; x)$, such that the following diagram on $G^{3} \times X$ is commutative:

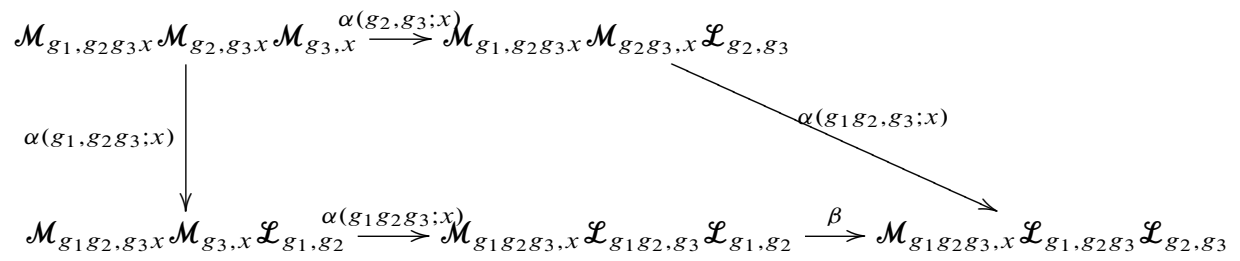

Here $\beta$ is induced by the structure of the 2-cocycle on $\mathscr{L}$ (we skipped the signs of tensor product for brevity).

In the case when the 2-cocycle $\mathscr{L}$ is trivial the definition (ii) (resp. (iii)) above reduces to the notion of a kernel representation (resp. a 1-cocycle with values in Pic $(X))$ considered in Section 3.4.

Examples. 1. Let $A$ be a commutative group scheme, $\mathscr{L}$ a biextension of $A \times A$ by $\mathbb{G}_{m}$. Then $\mathscr{L}$ has a natural structure of a 2-cocycle of $A$ with values in $\operatorname{Pic}(S)$.

2. If $f: G \rightarrow G^{\prime}$ is a homomorphism of group schemes over $S$ and $\mathscr{L}^{\prime}$ is a 2-cocycle of $G^{\prime}$ with values in $\operatorname{Pic}(S)$ then $(f \times f)^{*} \mathscr{L}^{\prime}$ has a natural structure of a 2-cocycle of $G$. In particular, by the previous example if we have a homomorphism $f$ from $G$ to a commutative group scheme $A$ and a biextension $\mathscr{L}$ of $A \times A$ by $\mathbb{G}_{m}$ then $(f \times f)^{*} \mathscr{L}$ has the structure of a 2-cocycle of $G$. More generally, if $A$ and $B$ are commutative group schemes and $\mathscr{L}$ is a biextension of $A \times B$ by $\mathbb{G}_{m}$ then for a pair of homomorphisms $f: G \rightarrow A, g: G \rightarrow B$ the pull-back $(f \times g)^{*} \mathscr{L}$ has a natural structure of a 2-cocycle of $G$.

Remark. The notion of a 2-cocycle with values in $\operatorname{Pic}(X)$ (and of the corresponding coboundaries) can be generalized to an arbitrary groupoid over $X$. However, we will not need this generalization.

Note that 2-cocycles of $G$ with values in $\operatorname{Pic}(S)$ form a commutative Picard category with respect to the tensor product operation. For any line bundle $\mathcal{M} \in$ $\operatorname{Pic}(G)$ we can form a 2-cocycle of $G$

$$
\Lambda(\mathcal{M})_{g_{1}, g_{2}}:=\mathcal{M}_{g_{1}}^{-1} \otimes \mathcal{M}_{g_{2}}^{-1} \otimes \mathcal{M}_{g_{1} g_{2}}
$$


with values in $\operatorname{Pic}(S)$. A coboundary with values in $\operatorname{Pic}(S)$ for a 2 -cocycle $\mathscr{L}$ is given by a line bundle $\mathcal{M}$ together with an isomorphism $\mathscr{L} \simeq \Lambda(\mathcal{M})^{-1}$ of 2-cocycles.

For a 2-cocycle $\mathscr{L}$ of $G$ with values in $\operatorname{Pic}(S)$ and a line bundle $\mathcal{M}$ on $G$ we have an equivalence from the category of projective kernel representations of $G$ over $X$ with the 2-cocycle $\mathscr{L} \otimes \Lambda(\mathcal{M})$ to the similar category for $\mathscr{L}$ sending $V$ to $V \otimes p_{G}^{*} \mathcal{M}$, where $p_{G}: X \times G \times X \rightarrow G$ is the projection.

Now assume that we have an action of $G$ on $X$. Generalizing the construction of Section 3.4, given a 2-cocycle $\mathscr{L}$ of $G$ with values in $\operatorname{Pic}(S)$ and a coboundary $\mathcal{M}$ for $\mathscr{L}$ with values in $\operatorname{Pic}(X)$, we can define a projective kernel representation of $G$ over $X$ by setting

$$
V_{\mathcal{M}}=\gamma_{G *}(\mathcal{M}),
$$

where $\gamma_{G}(g, x)=(g x, g, x)$. For $\mathcal{N} \in \operatorname{Pic}(G)$ the line bundle $\mathcal{M} \otimes p_{1}^{*} \mathcal{N} \in$ $\operatorname{Pic}(G \times X)$ acquires a natural structure of a coboundary for $\mathscr{L} \otimes \Lambda(\mathcal{N})^{-1}$ with values in $\operatorname{Pic}(X)$. The corresponding projective kernel representation is

$$
V_{\mathcal{M} \otimes p_{1}^{*} \mathcal{N}} \simeq V_{\mathcal{M}} \otimes p_{G}^{*} \mathcal{N} .
$$

Example. Let $A$ be an abelian variety over a field $k, \hat{A}$ the dual abelian variety. Set $X_{A}=A \times \hat{A}$. There is a natural homomorphism from the group $X_{A}(k)$ to the group of autoequivalences of $D^{b}(A)$ (viewed up to an isomorphism) such that $A(k)$ acts by translations and $\hat{A}(k)$ acts by tensoring with line bundles. Let us give a categorified version of this picture that also works in the relative setting (cf. [42], Sec. 3). Assume now that $A$ is an abelian scheme over $S, \hat{A}$ is the dual abelian scheme, and $X_{A}=A \times_{S} \hat{A}$. Then we are going to construct a projective kernel representation of $X_{A}$ over $A$ with the 2-cocycle $\mathscr{L}_{A}=p_{32}^{*} \mathcal{P}$, where $\mathcal{P}$ is the Poincaré line bundle on $X_{A}$ and $p_{32}: X_{A}^{2}=(A \times \hat{A})^{2} \rightarrow A \times \hat{A}$ sends $\left(x_{1}, \xi_{1}, x_{2}, \xi_{2}\right)$ to $\left(x_{2}, \xi_{1}\right)$. Namely, if we let $X_{A}$ act on $A$ by translations (with $\hat{A}$ acting trivially), then we have a natural coboundary $\mathcal{M}$ for $\mathscr{L}_{A}$ with values in $\operatorname{Pic}(A)$ given by

$$
\mathcal{M}_{(x, \xi ; y)}=\mathcal{P}_{y, \xi},
$$

where $(x, \xi ; y) \in(A \times \hat{A}) \times A$. Indeed, the biextension structure on $\mathcal{P}$ gives rise to an isomorphism

$$
\begin{aligned}
\mathcal{M}_{x_{1}, \xi_{1} ; x_{2}+y} \otimes \mathcal{M}_{x_{2}, \xi_{2} ; y} & =\mathcal{P}_{x_{2}+y, \xi_{1}} \otimes \mathcal{P}_{y, \xi_{2}} \\
& \simeq \mathcal{P}_{y, \xi_{1}+\xi_{2}} \otimes \mathcal{P}_{x_{2}, \xi_{1}}=\mathcal{M}_{x_{1}+x_{2}, \xi_{1}+\xi_{2} ; y} \otimes \mathcal{P}_{x_{2}, \xi_{1}} .
\end{aligned}
$$

Therefore, we have the corresponding projective kernel representation of $X_{A}$ over $A$ given by

$$
V(A):=V_{\mathcal{M}}=(x+y, x, \xi, y)_{*} \mathcal{M} \in \operatorname{Coh}\left(A \times X_{A} \times A\right),
$$

where $(x, \xi, y) \in A \times \hat{A} \times A$. 
We have the following version of Proposition 3.2.5 for projective representations. The proof is analogous, so we skip it.

Proposition 3.7.1. Let $X$ and $Y$ be schemes over $S$, and let $(\mathcal{P}, \mathcal{Q}, \alpha, \beta)$ be adjoint kernel data, where $\mathcal{P} \in D_{\mathrm{qc}}(X \times Y)$ and $\mathcal{Q} \in D_{\mathrm{qc}}(Y \times X)$. Let also $G$ be a group scheme, and let $V$ be a kernel projective representation of $G$ over $X$ with a 2 -cocycle $\mathscr{L}$. Then $\mathcal{Q}{ }^{\circ} V{ }_{X} \mathcal{P}$ has a natural structure of a kernel projective representation of $G$ over $Y$ with the same 2-cocycle. This correspondence is compatible with restriction under homomorphisms of group schemes and with the functors of the form $V \mapsto V \otimes p_{G}^{*} \mathcal{M}$ for $\mathcal{M} \in \mathcal{P i c}(G)$ (where $\mathscr{L}$ gets replaced by $\left.\mathscr{L} \otimes \delta \mathcal{M}\right)$. Similar assertions hold if $G$ is a formal group scheme, $X$ and $Y$ are proper over $S$, and $V \in D_{G, \overrightarrow{\mathrm{c}}}(X \times G \times X)$.

Recall that the Fourier-Mukai transform $D_{\mathrm{qc}}(A) \simeq D_{\mathrm{qc}}(\hat{A})$ corresponds to taking as $\mathcal{P}$ the Poincaré line bundle on $A \times \hat{A}$ and $Q=\mathcal{P}^{-1} \otimes p_{\hat{A}}^{*} \omega_{\hat{A} / S}[g]$ on $\hat{A} \times A$, where $p_{\hat{A}}$ is the projection to $\hat{A}, g$ is the relative dimension of $A / S$. Using the fact that this transform exchanges the operation of translation on $A$ and of tensoring with the corresponding line bundle on $A$, we can easily calculate the Fourier-Mukai dual of the projective kernel representation of $X_{A}=A \times \hat{A}$ over $A$ considered above.

Proposition 3.7.2. The Fourier-Mukai dual of the projective representation $V(A)$ of the group $X_{A}$ is given by

$$
\mathcal{Q}_{A} V(A) \circ_{A} \mathcal{P} \simeq p_{X}^{*} \mathcal{P}^{-1} \otimes\left(\operatorname{id}_{\hat{A}} \times \kappa \times \operatorname{id}_{\hat{A}}\right)^{*} V(\hat{A}),
$$

where $\kappa$ is the isomorphism

$$
\kappa: X_{A}=A \times \hat{A} \rightarrow X_{\hat{A}}=\hat{A} \times A:(x, \xi) \mapsto(\xi,-x),
$$

and $p_{X}: \hat{A} \times X_{A} \times \hat{A} \rightarrow X_{A}$ is the projection. The isomorphism (3.7.3) is compatible with the kernel projective representation structures on $V(A)$ and $V(\hat{A})$ via the isomorphism $\kappa: X_{A} \stackrel{\sim}{\longrightarrow} X_{\hat{A}}$ and the isomorphism

$$
\Lambda(\mathcal{P}) \otimes(\kappa \times \kappa)^{*} \mathscr{L}_{\hat{A}} \simeq \mathscr{L}_{A}
$$

of 2-cocycles of $X_{A}$.

Proof. Let us consider two simpler kernel representations related to $V(A)$ : the one corresponding to the action of translations, and the one corresponding to tensoring with line bundles associated with points in $\hat{A}$. The first is the kernel representation of $A$ (viewed as a group scheme) over $A$ given by

$$
V_{t}(A):=(x+y, x, y)_{*} \mathcal{O}_{A \times A} \in \operatorname{Coh}(A \times A \times A),
$$


where $(x, y) \in A \times A$. The second is the kernel representation of $\hat{A}$ over $A$ given by

$$
V_{\otimes}(A)=(x, \xi, x)_{*} \mathcal{P} \in \operatorname{Coh}(A \times \hat{A} \times A),
$$

where $x \in A, \xi \in \hat{A}$. We have natural isomorphisms

$$
\begin{aligned}
& V_{t}(A) \circ_{A} V_{\otimes}(A) \simeq V(A), \\
& V_{\otimes}(A) \circ_{A} V_{t}(A) \simeq p_{X}^{*} \mathcal{P} \otimes V(A),
\end{aligned}
$$

which are compatible with the structures of kernel representations on $V_{t}(A)$ and $V_{\otimes}(A)$ and with the structure of a projective kernel representation on $V(A)$ in the following sense. Combining (3.7.4) and (3.7.5) we get the following "commutation" relation:

$$
V_{\otimes}(A) \circ_{A} V_{t}(A) \simeq p_{23}^{*} \mathcal{P} \otimes V_{t}(A) \circ_{A} V_{\otimes}(A) .
$$

Now the compatibility asserts that the projective kernel representation structure on $V(A)$ is given by the composition

$$
\begin{aligned}
V(A) \circ_{A} V(A) & \simeq V_{t}(A) \circ_{A} V_{\otimes}(A) \circ_{A} V_{t}(A) \circ_{A} V_{\otimes}(A) \\
& \simeq p_{23}^{*} \mathcal{P} \otimes V_{t}(A) \circ_{A} V_{t}(A) \circ_{A} V_{\otimes}(A) \circ_{A} V_{\otimes}(A) \\
& \rightarrow p_{23}^{*} \mathcal{P} \otimes V_{t}(A) \circ_{A} V_{\otimes}(A) \simeq p_{23}^{*} \mathcal{P} \circ V(A),
\end{aligned}
$$

where the first and the last isomorphisms are induced by (3.7.4), the second isomorphism is induced by (3.7.6), and the arrow is given by the kernel representation structures on $V_{t}(A)$ and on $V_{\otimes}(A)$. Thus, it is enough to compute the Fourier duals of the kernel representations $V_{t}(A)$ and $V_{\otimes}(A)$. Using the relation $\mathcal{Q} \circ_{A} \mathcal{P} \simeq \Delta_{*} \hat{A}$ and the biextension structure of $\mathcal{P}$, one gets the following isomorphisms of kernel representations:

$$
\begin{aligned}
\mathcal{Q} \circ_{A} V_{\otimes}(A) \circ_{A} \mathcal{P} & \simeq V_{t}(\hat{A}), \\
\mathcal{Q} \circ_{A} V_{t}(A) \circ_{A} \mathcal{P} & \simeq\left(\operatorname{id}_{\hat{A}} \times[-1]_{A} \times \operatorname{id}_{\hat{A}}\right)^{*} V_{\otimes}(\hat{A}) .
\end{aligned}
$$

One can check that this indeed leads to (3.7.3).

We are going to use the above result to calculate the Fourier-Mukai duals of some kernel representations obtained from $V(A)$ by restricting to subgroups over which the corresponding 2-cocycle becomes a coboundary.

Definition. Let $G$ be a group scheme (resp. a formal group scheme) over $S, A$ an abelian scheme over $S$. A $G$-twisting data $T=\left(f, f^{\prime}, \alpha, \iota\right)$ for $A$ consists of homomorphisms $f: G \rightarrow A, f^{\prime}: G \rightarrow \hat{A}$, and of a line bundle $\alpha$ over $G$ equipped with an isomorphism

$$
\iota: \Lambda(\alpha) \simeq\left(f \times f^{\prime}\right)^{*} \mathcal{P}
$$


of 2-cocycles of $G$ with values in $\operatorname{Pic}(S)$, where $\mathcal{P}$ is the Poincaré biextension of $A \times \hat{A}$. If $T=\left(f, f^{\prime}, \alpha, \iota\right)$ is a $G$-twisting data for $A$ then the dual $G$-twisting data for $\hat{A}$ is $\hat{T}=\left(f^{\prime},-f, \hat{\alpha}, \iota^{\prime}\right)$, where

$$
\hat{\alpha}=\alpha \otimes\left(f, f^{\prime}\right)^{*} \mathcal{P}^{-1},
$$

and $\iota^{\prime}$ is induced by $\iota$.

Note that the 2-cocycle $\left(f \times f^{\prime}\right)^{*} \mathcal{P}$ of $G$ is the pull-back under $\left(f, f^{\prime}\right): G \rightarrow X_{A}$ of the natural 2-cocycle $\mathscr{L}_{A}=p_{32}^{*} \mathcal{P}$ of $X_{A}$. Hence, the coboundary (3.7.1) for $\mathscr{L}_{A}$ with values in $\operatorname{Pic}(A)$ together with the isomorphism $\iota$ induce a 1-cocycle of $G$ with values in $\operatorname{Pic}(A)$. The underlying line bundle on $G \times A$ is

$$
\mathscr{L}(T):=p_{1}^{*} \alpha \otimes\left(\mathrm{id} \times f^{\prime}\right)^{*} \mathcal{P} .
$$

It is easy to see that a 1-cocycle $\mathscr{L}$ of $G$ with values in $\operatorname{Pic}(A)$ appears in this way if and only if $\left.\mathscr{L}\right|_{g \times A_{S}} \in \operatorname{Pic}^{0}\left(A_{S}\right)$ for every geometric point $g$ of $G$ (where $s$ is the image of $g$ in $S$ ). Indeed, in this case we can write $\mathscr{L}$ in the form $p_{1}^{*} \alpha \otimes\left(\mathrm{id} \times f^{\prime}\right)^{*} \mathcal{P}$ for some line bundle $\alpha$ over $G$ and some morphism $f^{\prime}: G \rightarrow \hat{A}$. Unraveling the cocycle condition we get that $f^{\prime}$ should be a homomorphism and $\alpha$ should satisfy $\Lambda(\alpha) \simeq\left(f \times f^{\prime}\right)^{*} \mathcal{P}$.

We set $\operatorname{Qcoh}_{G}^{T}(A)=\operatorname{Qcoh}_{G}^{\mathscr{L}(T)}(A)\left(\operatorname{resp} \cdot \operatorname{Coh}_{G}^{T}(A)=\operatorname{Coh}_{G}^{\mathscr{L}(T)}(A)\right)$. Note that by Corollary 3.4.5, we have $\operatorname{Qcoh}_{G}^{T}(A) \simeq \mathcal{A}_{A}^{G}(\mathscr{L}(T))-\bmod \left(\operatorname{resp} . \operatorname{Coh}_{G}^{T}(A) \simeq\right.$ $\mathcal{A}_{A}^{G}(\mathscr{L}(T))$-mod $\left.{ }^{\mathrm{c}}\right)$. The following result generalizes Theorem 15.2 of [43].

Theorem 3.7.3. Assume that $S$ is semi-separated. The kernel representations of $G$ over $A$ and $\hat{A}$ associated with $\mathscr{L}(T)$ and $\mathscr{L}(\widehat{T})$ are Fourier-Mukai dual to each other. Hence, if $G$ is a finite flat group scheme over $S$ then the corresponding pure kernel algebras $\mathcal{A}_{A}^{G}(\mathscr{L}(T))$ and $\mathscr{A}_{\hat{A}}^{G}(\mathscr{L}(\widehat{T}))$ are also Fourier-Mukai dual, and we get exact equivalences

$$
\begin{aligned}
& D\left(\operatorname{Qcoh}_{G}^{T}(A)\right) \simeq D\left(\operatorname{Qcoh}_{G}^{\widehat{T}}(\hat{A})\right), \\
& D^{b}\left(\operatorname{Coh}_{G}^{T}(A)\right) \simeq D^{b}\left(\operatorname{Coh}_{G}^{\widehat{T}}(\hat{A})\right) .
\end{aligned}
$$

The first of these equivalences also holds if $S=\operatorname{Spec}(k)$, where $k$ is a field and $G$ is a formal group scheme, ldu-pf over $k$, such that the action of $\operatorname{Gal}(\bar{k} / k)$ on $G(\bar{k})$ factors through $\mathrm{Gal}\left(k^{\prime} / k\right)$ for some finite field extension $k \subset k^{\prime}$.

Proof. Let us denote by $\left.V(A)\right|_{G} \in \operatorname{Coh}(A \times G \times A)\left(\operatorname{resp} .\left.V(\hat{A})\right|_{G} \in \operatorname{Coh}(\hat{A} \times G \times \hat{A})\right)$ the restriction of the projective kernel representation $V(A)$ of $X_{A}$ over $A$, via the homomorphism $\left(f, f^{\prime}\right): G \rightarrow X_{A}$ (resp. $\left.\left(f^{\prime},-f\right): G \rightarrow X_{\hat{A}}\right)$. Then we have natural isomorphisms of kernel representations of $G$

$$
\left.V_{\mathscr{L}(T)} \simeq p_{G}^{*} \alpha \otimes V(A)\right|_{G},\left.\quad V_{\mathscr{L}(\widehat{T})} \simeq p_{G}^{*} \hat{\alpha} \otimes V(\hat{A})\right|_{G} .
$$


Hence, by Proposition 3.7.2,

$$
\left.\mathcal{Q}_{{ }_{A}} V_{\mathscr{L}(T)}{ }^{\circ} \mathcal{P} \simeq p_{G}^{*}\left(\alpha \otimes\left(f, f^{\prime}\right)^{*} \mathcal{P}^{-1}\right) \otimes V(\hat{A})\right|_{G} \simeq V_{\mathscr{L}(\widehat{T})} .
$$

By Proposition 3.2.5, this implies the isomorphism of kernel algebras

$$
\mathcal{Q} \circ_{A} \mathcal{A}_{A}^{G}(\mathscr{L}(T)) \circ_{A} \mathcal{P} \simeq \mathcal{A}_{\hat{A}}^{G}(\mathscr{L}(\widehat{T})) .
$$

Now the first equivalence of categories follows from Theorem 2.5.1 (i). Note that we can apply it because the kernel algebras $\mathcal{A}_{A}^{G}(\mathscr{L}(T))$ and $\mathcal{A}_{\hat{A}}^{G}(\mathscr{L}(\widehat{T}))$ are pure and of affine type by Proposition 3.6.2. To deduce the second equivalence of categories we use Theorem 2.5.1 (iii) (recall that if $G$ is finite then the kernel algebra $\mathcal{A}_{A}^{G}(\mathscr{L}(T)$ ) is finite).

Remarks. 1. Every $\mathbb{G}_{m}$-gerbe over an abelian variety $A$ over a field $k$ of characteristic zero arises from some twisting data as above. Indeed, as was observed in [13], every element $e \in H^{2}\left(A, \mathbb{G}_{m}\right)$ has the trivial pull-back to $H^{2}\left(B, \mathbb{G}_{m}\right)$ for some isogeny $\pi: B \rightarrow A$. Therefore, $e$ is associated with some 1 -cocycle $\mathscr{L}$ of $G=\operatorname{ker}(\pi)$ with values in $\operatorname{Pic}(B)$. The corresponding homomorphism $G(\bar{k}) \rightarrow N S(\bar{B})$ to the Néron-Severi group of $\bar{B}=B \otimes_{k} \bar{k}$ is trivial, since $N S(\bar{B})$ has no torsion. Therefore, $\left.\mathscr{L}\right|_{g \times \bar{B}} \in \operatorname{Pic}^{0}(\bar{B})$ for every $g \in G(\bar{k})$. As we have seen above, this implies that $\mathscr{L}=\mathscr{L}(T)$ for some $G$-twisting data $T$.

2. Whenever we have a $G$-twisting data $T$ such that both $f: G \rightarrow A$ and $f^{\prime}: G \rightarrow \hat{A}$ are injective, Theorem 3.7.3 gives a derived equivalence of categories of twisted coherent sheaves on $A / f(G)$ and on $\hat{A} / f^{\prime}(G)$. It would be interesting to try to describe all exact equivalences between derived categories of twisted coherent sheaves over abelian varieties by generalizing the picture of [41].

Example. Let us explain how the equivalences between modules over algebras of twisted differential operators ( $t d o$ 's) considered in [44] fit the above theorem. Let $A$ be an abelian variety over a field $k$ of characteristic zero, and let $G$ be a formal group scheme over $k$, isomorphic as a formal scheme to $\operatorname{Spf}\left(k\left[\left[t_{1}, \ldots, t_{n}\right]\right]\right)$. Then the kernel algebra $\mathcal{A}=\mathcal{A}_{A}^{G}(\mathscr{L}(T))$ over $A$ associated with a $G$-twisting data $T=\left(f, f^{\prime}, \alpha, \iota\right)$ for $A$ is a $D$-algebra (see [7], and Example 1 of Section 2.2 on p. 180). Furthermore, the natural filtration of $\omega_{G}$ (given by the infinitesimal neighborhoods of zero) induces an exhaustive algebra filtration $\mathcal{A}_{0} \subset \mathcal{A}_{1} \subset \cdots$ such that the associated graded algebra is isomorphic to the commutative $\mathcal{O}_{A}$-algebra $S\left(L_{G}\right) \otimes \mathcal{O}_{A}$, where $L_{G}$ is the Lie algebra of $G$ and $S\left(L_{G}\right)$ is the symmetric algebra on $L_{G}$. According to Lemma 5.1 of [44], such a $D$-algebra $\mathscr{A}$ is the universal enveloping algebra $U^{0}(\widetilde{L})$ corresponding to a structure of a Lie algebroid on $L_{G} \otimes \mathcal{O}_{A}$ and a central extension $\widetilde{L}$ of this Lie algebroid by $\mathcal{O}_{A}$. The Fourier-Mukai duality of such algebras (and derived categories of modules over them) developed in Sec. 7 of [44] matches the duality picture obtained from Theorem 3.7.3. Note that the algebra $\mathcal{A}_{A}^{G}(\mathscr{L}(T))$ is a tdo if and only if the tangent map $d f: L_{G} \rightarrow L_{A}$ is an isomorphism, where $L_{A}$ is 
the Lie algebra of $A$ (see Sec. 7 of [44]). In this case $f$ gives an isomorphism of $G$ with the formal group obtained from $A$.

Here is an example of dual twisting data that will be relevant for the next section.

Corollary 3.7.4. Let $A$ be a abelian scheme over a semi-separated scheme $S$, and let $G$ and $H$ be finite flat commutative group schemes over $S$ equipped with homomorphisms $f: G \rightarrow A, f^{\prime}: H \rightarrow \hat{A}$ along with a trivialization of the biextension $\left(f \times f^{\prime}\right)^{*} \mathcal{P}$ of $G \times H$, where $\mathcal{P}$ is the Poincaré biextension of $A \times \hat{A}$. We can view the line bundle $\mathscr{L}\left(f^{\prime}\right)=\left(p_{3}, f^{\prime} p_{2}\right)^{*} \mathcal{P}$ on $G \times H \times A$ as a 1 -cocycle of $G \times H$ with values in $\operatorname{Pic}(A)$ (where $H$ acts trivially on $A$ ), and the line bundle $\mathscr{L}(f)=\left(f p_{1}, p_{3}\right)^{*} \mathcal{P}$ on $G \times H \times \hat{A}$ as a 1-cocycle of $G \times H$ with values in $\mathcal{P} i c(\hat{A})$ (where $G$ acts trivially on $\hat{A})$. Then the kernel algebras $\mathcal{A}_{A}^{G \times H}\left(\mathscr{L}\left(f^{\prime}\right)\right)$ and $\mathcal{A}_{\hat{A}}^{G \times H}(\mathscr{L}(f))$ are Fourier-Mukai dual to each other, so we have exact equivalences of categories

$$
\begin{aligned}
& D\left(\mathrm{Q} \operatorname{coh}_{G \times H}^{\mathscr{L}\left(f^{\prime}\right)}(A)\right) \simeq D\left(\operatorname{Qcoh}_{G \times H}^{\mathscr{L}(f)}(\hat{A})\right), \\
& D^{b}\left(\operatorname{Coh}_{G \times H}^{\mathscr{L}\left(f^{\prime}\right)}(A)\right) \simeq D^{b}\left(\operatorname{Coh}_{G \times H}^{\mathscr{L}(f)}(\hat{A})\right) .
\end{aligned}
$$

The first of these equivalences also holds if $S=\operatorname{Spec}(k)$, where $k$ is a field, and $G$ (resp. $H$ ) is a formal group scheme, ldu-pf over $k$, such that the action of $\operatorname{Gal}(\bar{k} / k)$ on $G(\bar{k})$ (resp. $H(\bar{k}))$ factors through $\mathrm{Gal}\left(k^{\prime} / k\right)$ for some finite field extension $k \subset k^{\prime}$.

Proof. By definition, the 1-cocycle $\mathscr{L}\left(f^{\prime}\right)$ of $G \times H$ with values in $\operatorname{Pic}(A)$ has the form $\mathscr{L}(T)$ for the $G \times H$-twisting data for $A$,

$$
T=\left(f p_{1}, f^{\prime} p_{2}, \mathcal{O}_{G \times H}, \iota\right),
$$

where $\iota$ is induced by the trivialization of $\left(f \times f^{\prime}\right)^{*} \mathcal{P}$ on $G \times H$. Similarly, $\mathscr{L}(f)=$ $\mathscr{L}(\widetilde{T})$, for the $G \times H$-twisting data for $\hat{A}$,

$$
\widetilde{T}=\left(f^{\prime} p_{2}, f p_{1}, \mathcal{O}_{G \times H}, \tilde{\iota}\right),
$$

with $\tilde{\imath}$ induced by the above trivialization. Note that $\widetilde{T}$ differs from the twisting data $\widehat{T}$ by the automorphism id $\times[-1]$ of $G \times H$. Hence, the kernel algebras on $\hat{A}$ associated with $\widetilde{T}$ and $\widehat{T}$ are isomorphic. It remains to use Theorem 3.7.3.

\section{Fourier-Mukai duality for orbi-abelian schemes and generalized 1-motives}

In this section we will apply the techniques of kernel algebras to get versions of the Fourier-Mukai equivalences in two situations. The first situation arises when we have a homomorphism $G \rightarrow E$, where $G$ is a finite flat commutative group scheme, $E$ is an extension of an abelian scheme by a finite flat commutative group scheme, and we consider the category of $G$-equivariant sheaves on $E$. It turns out 
that one can construct a dual data of this kind and an analog of the Fourier-Mukai functor. The second situation is the generalization of the one considered by Laumon in [33]: here we work over a field $k$ of characteristic zero, $G$ is a formal $k$-group (see the beginning of Section 3 for our conventions on formal $k$-groups), and $E$ is a commutative algebraic group over $k$ (i.e., a commutative group scheme of finite type over $k$ ).

We start by studying in sections 4.1 and 4.2 the duality in the particular case of the first situation when the base scheme is a field, since in this case a nice duality functor exists on certain derived category containing both abelian varieties and finite commutative group schemes. Then we will proceed to more general setups mentioned above.

4.1. Duality functor on the derived category of commutative proper group schemes over a field. Let $k$ be a field. For a commutative algebraic group $X$ over $k$ we denote by $X_{0}$ its connected component of 1 taken with reduced scheme structure (this is denoted as $\mathrm{CR}(X)$ in [39]). Also for an integer $N \neq 0$ we denote by $X_{N}$ the kernel of the map $[N]_{X}: X \rightarrow X: x \mapsto N x$.

Lemma 4.1.1. The following conditions for a commutative algebraic group $X$ over $k$ are equivalent:

(a) $X$ is proper;

(b) $X_{0}$ is an abelian variety;

(c) $X$ is isomorphic to a subgroup scheme of an abelian variety.

Proof. The equivalence of (a) and (b) is well known. It is clear that (c) implies (a). To prove (b) $\Longrightarrow$ (c) we use the fact that every finite group scheme can be embedded into an abelian variety as a subgroup scheme (this is due to Raynaud, see [36], Thm. A.6). Let $X$ be any commutative proper group scheme. Then there exists an integer $N>0$ such that $X / X_{0}$ is annihilated by $N$. Hence, $X_{0}=N X_{0} \subset N X \subset X_{0}$ so that $N X=X_{0}$. It follows that $X / X_{N} \simeq X_{0}$ is an abelian variety. Now pick an embedding $X_{N} \subset A$, where $A$ is an abelian variety. Then $X$ is a subgroup scheme of the induced extension of $X_{0}$ by $A$, which is an abelian variety.

Let $\mathcal{E}_{k}^{\mathrm{pr}}$ denote the category of commutative proper group schemes over $k$. This is a full subcategory of the category $\mathscr{E}_{k}$ of all commutative algebraic groups over $k$. It is well known that $\mathscr{S}_{k}$ is an abelian category (see [22], 5.4). In the case of an algebraically closed field a nice exposition of the properties of this category can be found in Chap. II of [39].

Lemma 4.1.2. The subcategory $\mathcal{E}_{k}^{\mathrm{pr}} \subset \mathcal{E}_{k}$ is a Serre subcategory. 
Proof. It is clear that $\mathscr{E}_{k}^{\mathrm{pr}}$ is closed under quotients and subgroup schemes. Assume that

$$
0 \rightarrow X \rightarrow Y \rightarrow Z \rightarrow 0
$$

is an exact sequence in $\mathscr{E}_{k}$ with $X, Z \in \mathcal{E}_{k}^{\mathrm{pr}}$. Then the morphism $Y \rightarrow Z$ is proper and $Z$ is proper, hence, $Y$ is proper.

For the theory of torsion pairs and tilting the reader can consult [30] (a concise exposition can be found also in Sec. 5.4 of [14]).

Lemma 4.1.3. Let $\mathcal{Y}_{k}^{\mathrm{f}}$ and $\mathcal{A V}_{k}$ be the subcategories of finite group schemes and abelian varieties in $\mathcal{E}_{k}^{\mathrm{pr}}$, respectively. Then $\left(\mathcal{A V}_{k}, \mathcal{Y}_{k}^{\mathrm{f}}\right)$ is a tilting torsion pair in $\mathcal{E}_{k}^{\mathrm{pr}}$.

Proof. Any morphism from an abelian variety to a finite group scheme is trivial. Also, by Lemma 4.1.1, for $X \in \mathcal{E}_{k}^{\mathrm{pr}}$ we have $X_{0} \in \mathcal{A V}_{k}$ and $\pi_{0} X:=X / X_{0} \in \mathscr{Y}_{k}^{\mathrm{f}}$, so $\left(\mathcal{A V}_{k}, \mathscr{Y}_{k}^{\mathrm{f}}\right)$ is a torsion pair. Applying Lemma 4.1.1 again we see that every object of $\mathscr{E}_{k}^{\mathrm{pr}}$ can be embedded into an object of $\mathcal{A V}_{k}$, i.e., our torsion pair is tilting.

Theorem 4.1.4. There exists an exact functor $\mathbb{D}: D^{b}\left(\mathcal{E}_{k}^{\mathrm{pr}}\right)^{\mathrm{opp}} \rightarrow D^{b}\left(\mathcal{E}_{k}^{\mathrm{pr}}\right)$ such that

(i) $\mathbb{D} \circ \mathbb{D} \simeq \mathrm{Id}$;

(ii) $\mathbb{D}(A) \simeq \hat{A}$ for an abelian variety $A$;

(iii) $\mathbb{D}(G) \simeq G^{*}[1]$ for a finite group scheme $G$.

Proof. The idea is to use resolutions in terms of abelian varieties. More precisely, the fact that $\left(\mathcal{A} \mathcal{V}_{k}, \mathscr{Y}_{k}^{\mathrm{f}}\right)$ is a tilting torsion pair in $\mathscr{Y}_{k}^{\mathrm{pr}}$ gives an equivalence $D^{b}\left(\mathcal{E}_{k}^{\mathrm{pr}}\right) \simeq$ $D^{b}\left(\mathcal{A V}_{k}\right)$, where $\mathcal{A V}_{k}$ is viewed as an exact category (see [14], Lemma 5.4.2; see also [8], Ex. 1.3.23 (iii)). Since the duality for abelian varieties preserves short exact sequences, it extends to an exact functor $\mathbb{D}: D^{b}\left(\mathcal{A V}_{k}\right) \rightarrow D^{b}\left(\mathcal{A V}_{k}\right)$. This proves (i) and (ii). Embedding a finite group scheme $G$ into an abelian variety and using the Isogeny Theorem (see [39], III.19.1) gives (iii).

Remark. It is easy to see that $\mathbb{D}$ exchanges the standard $t$-structure on $D^{b}\left(\mathscr{E}_{k}^{\mathrm{pr}}\right)$ with the tilted $t$-structure associated with the torsion pair $\left(\mathcal{A} \mathcal{V}_{k}, \mathscr{Y}_{k}^{\mathrm{f}}\right)$. More precisely, for every $X \in \mathscr{G}_{k}^{\mathrm{pr}}$ the duality sends the canonical exact sequence

$$
0 \rightarrow X_{0} \rightarrow X \rightarrow \pi_{0} X \rightarrow 0,
$$

where $X_{0}$ is an abelian variety, to the exact triangle

$$
\left(\pi_{0} X\right)^{*}[1] \rightarrow \mathbb{D}(X) \rightarrow \hat{X}_{0} \rightarrow\left(\pi_{0} X\right)^{*}[2]
$$

so that $\left(\pi_{0} X\right)^{*}[1] \simeq \tau_{\leq-1} \mathbb{D}(X)$ and $\hat{X}_{0} \simeq \tau_{\geq 0} \mathbb{D}(X)$. Thus, we have

$$
\begin{aligned}
H^{-1} \mathbb{D}(X) & \simeq\left(\pi_{0} X\right)^{*}, \\
H^{0} \mathbb{D}(X) & \simeq \hat{X}_{0} .
\end{aligned}
$$


4.2. Orbi-abelian varieties. Let $k$ be a field.

Definition. An orbi-abelian variety over $k$ is an object $K$ of $D^{b}\left(\mathscr{E}_{k}^{\mathrm{pr}}\right)$ such that $H^{-1} K$ is a finite group scheme and $H^{i} K=0$ for $i \notin\{-1,0\}$.

Lemma 4.2.1. (i) For any complex $K$ in $\mathcal{E}_{k}^{\mathrm{pr}}$ concentrated in degrees -1 and 0 such that $H^{-1} K$ is finite, there exists a quasiisomorphic subcomplex of the form $[G \rightarrow X]$, where $G$ is a finite group scheme. In particular, every orbi-abelian variety can be presented by a complex $[G \rightarrow X]$ with $G$ finite.

(ii) An object $K \in D^{b}\left(\mathcal{E}_{k}^{\mathrm{pr}}\right)$ is an orbi-abelian variety if and only if $\mathbb{D}(K)$ is. Furthermore, we have natural isomorphisms

$$
\begin{aligned}
\left(H^{0} \mathbb{D}(K)\right)_{0} & \simeq \widehat{\left(H^{0} K\right)_{0}}, \\
H^{-1} \mathbb{D}(K) & \simeq\left(\pi_{0} H^{0} K\right)^{*} .
\end{aligned}
$$

Proof. (i) Let $K=[i: Y \rightarrow X]$, where $\operatorname{ker}(i)$ is finite. We claim that there exists a homomorphism $p: X \rightarrow Y_{0}$ such that $p \circ i=[N]_{Y}$ for some $N>0$ (where $\left.[N]_{Y} Y \subset Y_{0}\right)$. Indeed, since $Y_{0}$ and $X_{0}$ are abelian varieties the assertion is true for the induced homomorphism $i_{0}: Y_{0} \rightarrow X_{0}$, so we can find $p_{0}: X_{0} \rightarrow Y_{0}$ such that $p_{0} \circ i_{0}=\left[N_{1}\right]_{Y_{0}}$ for some $N_{1}>0$. If $N_{2}=\left|X / X_{0}\right|$ then the multiplication by $N_{2}$ maps $X$ to $X_{0}$, so we can view $\tilde{p}=N_{2} p_{0}$ as a homomorphism $X \rightarrow Y_{0}$. Finally, the homomorphisms $\tilde{p} \circ i$ and $\left[N_{1} N_{2}\right]_{Y}$ agree on $Y_{0}$, hence

$$
N_{3} \tilde{p} \circ i=\left[N_{1} N_{2} N_{3}\right]_{Y},
$$

where $N_{3}=\left|Y / Y_{0}\right|$. Thus, we can set $p=N_{3} \tilde{p}, N=N_{1} N_{2} N_{3}$, and our claim follows. In this situation $K$ is quasi-isomorphic to the subcomplex $\left[i^{\prime}: Y_{N} \rightarrow \operatorname{ker}(p)\right]$, where $i^{\prime}$ is induced by $i$.

(ii) Let $K=[G \rightarrow X]$ be an orbi-abelian variety, where $G$ is a finite group scheme. Then we have an exact triangle

$$
X \rightarrow K \rightarrow G[1] \rightarrow X[1]
$$

The dual of this triangle is

$$
G^{*} \rightarrow \mathbb{D}(K) \rightarrow \mathbb{D}(X) \rightarrow G^{*}[1] .
$$

The corresponding long exact sequence of cohomology shows that $H^{i} \mathbb{D}(K)=0$ for $i \notin\{-1,0\}$ and that $H^{-1} \mathbb{D}(K)$ is a subgroup scheme in $H^{-1} \mathbb{D}(X) \simeq\left(\pi_{0} X\right)^{*}$ (see (4.1.1)). Hence, $H^{-1} \mathbb{D}(K)$ is finite, so $\mathbb{D}(K)$ is an orbi-abelian variety. The same exact sequence shows that

$$
H^{-1} \mathbb{D}(K) \simeq \operatorname{ker}\left(\left(\pi_{0} X\right)^{*} \rightarrow G^{*}\right) \simeq \operatorname{coker}\left(G \rightarrow \pi_{0} X\right)^{*} \simeq\left(\pi_{0} H^{0} K\right)^{*} .
$$


On the other hand, by (4.1.2), the abelian variety $H^{0} \mathbb{D}(K)_{0}$ is dual to $H^{0} \mathbb{D} H^{0} \mathbb{D}(K)$. Dualizing the standard exact triangle

$$
H^{-1} \mathbb{D}(K)[1] \rightarrow \mathbb{D}(K) \rightarrow H^{0} \mathbb{D}(K) \rightarrow \cdots
$$

and passing to cohomology we get an exact sequence

$$
0 \rightarrow H^{0} \mathbb{D} H^{0} \mathbb{D}(K) \rightarrow H^{0} K \rightarrow\left(H^{-1} \mathbb{D}(K)\right)^{*} \rightarrow \cdots,
$$

which induces an isomorphism $H^{0} \mathbb{D} H^{0} \mathbb{D}(K) \simeq\left(H^{0} K\right)_{0}$.

Here is a simple way to realize the dual $\mathbb{D}(K)$ to an orbi-abelian variety $K$ concretely. Let $K=[G \rightarrow X]$ as in Lemma 4.2.1 (i). We can pick a finite subgroup $H \subset X$ such that $A=X / H$ is an abelian variety (e.g., one can take $H=X_{N}$, where $X / X_{0}$ is annihilated by $\left.N\right)$. Note that the dual of $L=[G \rightarrow A]$ has only one cohomology due to an exact triangle

$$
G^{*} \rightarrow \mathbb{D}(L) \rightarrow \hat{A} \rightarrow G^{*}[1] .
$$

On the other hand, dualizing the exact triangle

$$
K \rightarrow L \rightarrow H[1] \rightarrow K[1]
$$

we get

$$
H^{*} \rightarrow \mathbb{D}(L) \rightarrow \mathbb{D}(K) \rightarrow H^{*}[1] .
$$

Thus, the complex $\left[H^{*} \rightarrow \mathbb{D}(L)\right]$ represents $\mathbb{D}(K)$.

Remark. If $k$ is algebraically closed of characteristic zero then the category $\mathscr{E}_{k}^{\mathrm{pr}}$ has homological dimension 1 (see [46], Thm. 10.1, or [39]), Sec. II.14, so every object of $D^{b}\left(\mathcal{E}_{k}^{\mathrm{pr}}\right)$ is isomorphic to the direct sum of its cohomologies. By duality, this implies that in this case every object of $\mathscr{E}_{k}^{\mathrm{pr}}$ is a direct sum of an abelian variety and a finite group scheme. Thus, in this case the duality for orbi-abelian varieties takes form

$$
\mathbb{D}(G[1] \oplus(H \oplus A)) \simeq H^{*}[1] \oplus\left(G^{*} \oplus \hat{A}\right),
$$

where $A$ is an abelian variety, $H$ and $G$ are finite commutative group schemes. Now assume that $k$ is algebraically closed of characteristic $p>0$. Let $\mathcal{E}_{k, 0}^{\mathrm{pr}} \subset \mathcal{E}_{k}^{\mathrm{pr}}$ denote the full subcategory consisting of proper groups $G$ such that $\operatorname{Hom}\left(\alpha_{p}, G\right)=0$. We claim that $\mathscr{E}_{k, 0}^{\mathrm{pr}}$ is a Serre subcategory in $\mathscr{E}_{k}^{\mathrm{pr}}$. Indeed, it is clear that $\mathscr{E}_{k, 0}^{\mathrm{pr}}$ is closed under extensions and passing to subobjects. To check that it is closed under quotients it suffices to consider quotients by elementary groups. But for such a group $G$ one has $\operatorname{Hom}\left(\alpha_{p}, G\right)=0$ if and only if $\operatorname{Ext}^{1}\left(\alpha_{p}, G\right)=0$ (see [39], Sec. II.14), and our claim follows. Note that the category $\mathcal{E}_{k, 0}^{\mathrm{pr}}$ also has homological dimension 1 (see [39], Sec. II.14), so the above remarks about the characteristic zero case apply as well to orbi-abelian varieties in $D^{b}\left(\mathscr{\Theta}_{k, 0}^{\mathrm{pr}}\right)$. 
4.3. Abstract duality setup. Let $\delta$ be a site. We denote by $\delta h \delta$ the category of sheaves of abelian groups on $\delta$. We assume that we have fixed a certain sheaf of abelian groups $\mathbf{G}$. We consider the associated duality functor

$$
\mathbb{D}: D^{b}\left(S h_{\mathcal{S}}\right) \rightarrow D^{+}\left(S h_{\mathcal{S}}\right): K \mapsto R \underline{\operatorname{Hom}}(K, \mathbf{G})[1] .
$$

Assume also that we have three full subcategories stable under extensions For, Aff, $\mathcal{A} b \subset S h_{\mathcal{S}}$ with the following properties:

(i) $\mathbb{D}(\mathcal{A} b) \subset \mathcal{A} b, \mathbb{D}(\mathcal{F} o r)[-1] \subset \mathcal{A} f f, \mathbb{D}(\mathcal{A} f f)[-1] \subset \mathcal{F}$ or;

(ii) for $K$ in one of the subcategories For, $\mathcal{A} f f$ or $\mathcal{A} b$, the natural morphism $K \rightarrow$ $\mathbb{D D}(K)$ is an isomorphism.

In this situation for $A \in \mathcal{A} b$ we set $\hat{A}:=\mathbb{D}(A)$, while for $G$ either in For or in $\mathcal{A} f f$ we set $G^{*}=\mathbb{D}(G)[-1]$. Note that (i) and (ii) imply that for $A \in \mathcal{A} b$ and for $G$ either in $\mathcal{F}$ or or in $\mathcal{A f f}$ one has $\operatorname{Hom}(A, G)=0$. Indeed, we have $\mathbb{D}(A) \in S h_{\mathcal{S}}$ and $\mathbb{D}(G) \in S h_{S}[1]$. Hence, $\operatorname{Hom}(\mathscr{D}(G), \mathscr{D}(A))=0$, and the required vanishing follows from (ii).

Definition. Given the data $\Delta=(\mathbf{G}, \mathcal{F}$ or, $\mathcal{A} f f, \mathcal{A} b)$ as above, we define a generalized 1-motive of type $\Delta$ as a complex $[G \rightarrow E]$ of sheaves of abelian groups on $S$ concentrated in degrees -1 and 0 such that $G \in \mathcal{F}$ or, and $E$ fits into an exact sequence

$$
0 \rightarrow L \rightarrow E \rightarrow A \rightarrow 0
$$

with $L \in \mathcal{A} f f$ and $A \in \mathcal{A} b$. Morphisms between generalized 1-motives as above are simply morphisms in the derived category $D^{b}\left(S h_{\mathcal{S}}\right)$.

We will consider the following two examples of this situation. The first is when $S$ is the fppf site of schemes over a given scheme $S, \mathbf{G}=\mathbb{G}_{m}$ is the multiplicative group, $A b$ consists of abelian schemes over $S$, while $\mathscr{F} o r=\mathcal{A f f}$ are finite flat commutative group schemes over $S$. We denote the corresponding duality type $\Delta_{S}^{\text {fin }}$ and call generalized 1-motives of type $\Delta_{S}^{\text {fin }}$ orbi-abelian schemes over $S$.

In the second example $S$ is the fppf site of affine schemes over a field $k$ of characteristic zero, $\mathbf{G}=\mathbb{G}_{m}, \mathcal{A} b$ consists of abelian varieties over $k$, For are formal $k$-groups (see our conventions in the beginning of Section 3 ), and $\mathcal{A} f f$ are affine commutative algebraic groups over $k$. We denote the corresponding duality type $\Delta_{k}^{\text {for }}$. To get the usual generalized 1-motives of Laumon [33] one would have to modify this type by taking $\mathcal{F}$ or to be formal $k$-groups without torsion and considering only connected groups in $\mathcal{A f f}$.

Sometimes it is convenient to view generalized 1-motives as a full subcategory in the category of Picard stacks by associating with $K=[G \rightarrow E]$ the Picard stack $\operatorname{ch}(K)$ (see Section 1.3 and [18], 1.4).

Proposition 4.3.1. If $K$ is a generalized 1-motive of type $\Delta$ then $\mathbb{D}(K)$ is also a generalized 1-motive of type $\Delta$ and the natural map $K \rightarrow \mathbb{D D}(K)$ is an isomorphism. 
Proof. Let $K=[G \rightarrow E]$, where $G \in$ For and $E$ is an extension of $A \in \mathcal{A} b$ by $L \in \mathcal{A} f f$. Set $\bar{K}=[G \rightarrow A]$. Then $\mathbb{D}(\bar{K})$ is an extension of $\hat{A}=\mathbb{D}(A)$ by $G^{*}=\mathbb{D}(G)[-1]$, and $\mathbb{D}(K)$ is represented by the complex $\left[L^{*} \rightarrow \mathbb{D}(\bar{K})\right]$. The second assertion now follows from the assumption (ii) on the data $\Delta$.

The duality of generalized 1-motives has a convenient interpretation in terms of biextensions. Recall that if $[Y \rightarrow X]$ and $\left[Y^{\prime} \rightarrow X^{\prime}\right]$ are complexes over $\delta h_{S}$ concentrated in degrees -1 and 0 then a biextension of $[Y \rightarrow X]$ and $\left[Y^{\prime} \rightarrow X^{\prime}\right]$ by $\mathbf{G}$ is a biextension of $X \times X^{\prime}$ by $\mathbf{G}$ equipped with trivialization of its pull-backs to $Y \times X^{\prime}$ and $X \times Y^{\prime}$ such that the induced trivialization of the pull-back to $Y \times Y^{\prime}$ are the same (see [19], 10.2). Such biextensions form a (commutative) Picard category and we denote by $\operatorname{Biext}^{1}\left([Y \rightarrow X],\left[Y^{\prime} \rightarrow X^{\prime}\right] ; \mathbf{G}\right)\left(\operatorname{resp} . \operatorname{Biext}^{0}\left([Y \rightarrow X],\left[Y^{\prime} \rightarrow X^{\prime}\right], \mathbf{G}\right)\right)$ the group of isomorphism classes in this category (resp. automorphism group of an object). These groups are isomorphic to $\operatorname{Ext}^{i}\left([Y \rightarrow X] \otimes\left[Y^{\prime} \rightarrow X^{\prime}\right], \mathbf{G}\right), i=0,1$, so they depend only on isomorphism classes of $[Y \rightarrow X]$ and $\left[Y^{\prime} \rightarrow X^{\prime}\right]$ in the derived category $D^{b}\left(S h_{s}\right)$ (see [19], 10.2.1).

Proposition 4.3.2. Let $K$ and $K^{\prime}$ be generalized 1-motives of type $\Delta$.

(i) One has a functorial isomorphism

$$
\operatorname{Hom}_{D^{b}\left(s h_{s}\right)}\left(K^{\prime}, \mathbb{D}(K)\right) \simeq \operatorname{Biext}^{1}\left(K, K^{\prime} ; \mathbf{G}\right) .
$$

(ii) For a presentation $K=[G \rightarrow E]$ let $\operatorname{EXT}([G \rightarrow E]$, G) denote the Picard stack of extensions of $[G \rightarrow E]$ by $\mathbf{G}$ in the category of complexes over $S h_{s}$. Equivalently, $\operatorname{EXT}([G \rightarrow E], \mathbf{G})$ classifies extensions of $E$ by $\mathbf{G}$ with trivialized pull-back to $G$. Then we have an isomorphism of Picard stacks

$$
\operatorname{ch} \mathbb{D}(K) \simeq \operatorname{EXT}(K, \mathbf{G}) .
$$

(iii) Let $K=[G \rightarrow E]$, where $E$ is an extension of $A \in \mathcal{A} b$ by $L \in \mathcal{A}$ ff, so that $\mathbb{D}(K)$ is represented by $\left[L^{*} \rightarrow \mathbb{D}(\bar{K})\right]$ with $\bar{K}=[G \rightarrow A]$. Then the biextension of $K \times \mathbb{D}(K)$ corresponding to the identity map $\mathrm{id}_{\mathbb{D}(K)}$ under the isomorphism of (i) (the Poincaré biextension) is represented by the pull-back to $E \times \mathbb{D}(\bar{K})$ of the similar biextension of $A \times \hat{A}$, where $\hat{A}=\mathbb{D}(A)$. This pull-back is equipped with natural trivializations along $G \times \mathbb{D}(\bar{K})$ and $E \times L^{*}$ that are compatible over $G \times L^{*}$.

Proof. (i) We have

$$
\begin{aligned}
\operatorname{Hom}\left(K^{\prime}, \mathbb{D}(K)\right)=\operatorname{Hom}\left(K^{\prime}, R \underline{\operatorname{Hom}}(K, \mathbf{G}[1])\right) \\
\simeq \operatorname{Hom}\left(K^{\prime} \otimes K, \mathbf{G}[1]\right)=\operatorname{Ext}^{1}\left(K^{\prime} \otimes K, \mathbf{G}\right),
\end{aligned}
$$

which is isomorphic to $\operatorname{Biext}^{1}\left(K, K^{\prime} ; \mathbf{G}\right)$ (see [19], 10.2.1).

(ii) By Lemma 1.3.1, we have

$$
\operatorname{EXT}(K, \mathbf{G}) \simeq \operatorname{HOM}(\operatorname{ch}(K), \operatorname{ch}(\mathbf{G}[1])) \simeq \operatorname{ch}\left(\tau_{\leq 0} R \underline{\operatorname{Hom}}(K, \mathbf{G}[1])\right),
$$


so the assertion follows from $R \underline{\operatorname{Hom}}(K, \mathbf{G}[1])=\mathbb{D}(K) \in D^{\leq 0}\left(s h_{s}\right)$.

(iii) Note that the Poincaré biextension of $K \times \mathbb{D}(K)$ corresponds to the canonical morphism $K \otimes \mathbb{D}(K) \rightarrow \mathbf{G}[1]$ via the isomorphism $\operatorname{Biext}^{1}(K, \mathbb{D}(K) ; \mathbf{G}[1]) \simeq$ $\operatorname{Ext}^{1}(K \otimes \mathbb{D}(K), \mathbf{G})$. Applying this to $\bar{K}$ and using the commutative diagram

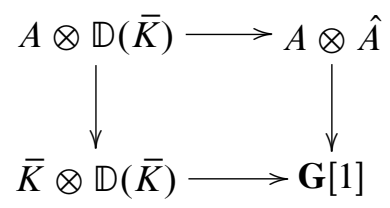

we see that the Poincaré biextension of $\bar{K} \times \mathbb{D}(\bar{K})$ is represented by the pull-back to $A \times \mathbb{D}(\bar{K})$ of the Poincaré biextension of $A \times \hat{A}$ (this pull-back is equipped with a trivialization over $G \times \mathbb{D}(\bar{K})$ ). Now the assertion follows in a similar way from the commutative diagram

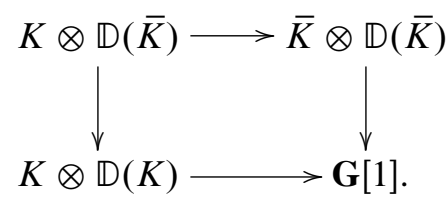

4.4. Fourier-Mukai transform. In this section we work with generalized 1-motives of type $\Delta=\left(\mathbb{G}_{m}, \mathcal{F}\right.$ or, $\left.\mathcal{A} f f, \mathcal{A} b\right)$, where $\Delta$ is either $\Delta_{S}^{\text {fin }}$ (orbi-abelian schemes) or $\Delta_{k}^{\text {for }}$. Thus, our generalized 1-motives are of the form $K=[G \rightarrow E]$, where either (i) $E$ is an extension of an abelian scheme by a finite flat commutative group scheme over $S, G$ is a finite flat commutative group scheme over $S$, or (ii) $E$ is a commutative algebraic group over a field $k$ of characteristic zero, $G$ is a formal $k$-group. Note that in case (ii) $E$ is an extension of an abelian variety by an affine commutative algebraic group. In both cases by a quasi-coherent sheaf on $K$ we mean a $G$-equivariant quasicoherent sheaf on $E$ (where $G$ acts on $E$ by translations). We denote the category of quasi-coherent sheaves by Qcoh $K$. In case (i) we can also consider the subcategory Coh $K$ of coherent sheaves. It is easy to check that up to equivalence the category Qcoh $K$ does not depend on a presentation $K=[G \rightarrow E]$. Indeed, this is clear in the case when we have surjective morphism of complexes $f:\left[G^{\prime} \rightarrow E^{\prime}\right] \rightarrow[G \rightarrow E]$ (i.e., both maps $f_{-1}: G^{\prime} \rightarrow G$ and $f_{0}: E^{\prime} \rightarrow E$ are surjective) such that $\operatorname{ker}\left(f_{-1}\right)$ maps isomorphically to $\operatorname{ker}\left(f_{0}\right)$ (cf. Lemma 3.4.3 - note that $\operatorname{ker}\left(f_{-1}\right) \simeq \operatorname{ker}\left(f_{0}\right)$ is a finite group scheme). The general case follows because of the following simple result.

Lemma 4.4.1. Let $[G \rightarrow E]$ and $\left[G^{\prime} \rightarrow E^{\prime}\right]$ be two presentations of the same generalized 1-motive $K$. Then there exists a third presentation $\left[G_{0} \rightarrow E_{0}\right]$ of $K$ equipped with surjective maps (quasi-isomorphisms) to $[G \rightarrow E]$ and $\left[G^{\prime} \rightarrow E^{\prime}\right]$. 
Proof. Let us define $E_{0}$ in the derived category of sheaves from the exact triangle

$$
E_{0} \rightarrow E \oplus E^{\prime} \rightarrow K \rightarrow E_{0}[1] .
$$

Using the octahedron axiom one can easily see that $E_{0}$ is an extension of $E$ by $G^{\prime}$ (resp. of $E^{\prime}$ by $G$ ), and that $\left[G \oplus G^{\prime} \rightarrow E_{0}\right]$ will be the required third presentation of $K$.

Let $K=[G \stackrel{f}{\rightarrow} E]$ be a generalized 1-motive of one of the two types above. By definition, $E$ is an extension of $A \in \mathcal{A} b$ by $L \in \mathcal{A}$ ff. Note that $A$ is an abelian scheme over $S$ (where $S=\operatorname{Spec}(k)$ in case (ii)). Let $\pi: E \rightarrow A$ denote the projection. Then we associate with the presentation $K=[G \rightarrow E]$ the kernel algebra

$$
\mathcal{A}(G \rightarrow E)=(\pi \times \pi)_{*} \mathcal{A}_{E}^{G}
$$

over $A$. By Corollary 3.3.2 and Lemma 2.2.6, the category Qcoh $K$ is equivalent to the category of $\mathcal{A}(G \rightarrow E)$-modules on $A$. In the case $\Delta=\Delta_{S}^{\text {fin }}$ the kernel algebra $\mathcal{A}(G \rightarrow E)$ is finite and we have an equivalence $\operatorname{Coh} K \simeq \mathcal{A}(K)$-mod ${ }^{c}$.

As we have seen before, the dual 1-motive is represented as $\mathbb{D}(K)=\left[L^{*} \rightarrow E^{\prime}\right]$, where $E^{\prime}=\mathbb{D}[G \rightarrow A]$ is an extension of $\hat{A}$ by $G^{*}$. Thus, denoting by $\rho: E^{\prime} \rightarrow \hat{A}$ the natural map we also have the corresponding kernel algebra

$$
\mathcal{A}\left(L^{*} \rightarrow E^{\prime}\right)=(\rho \times \rho)_{*} \mathcal{A}_{E^{\prime}}^{L^{*}}
$$

over $\hat{A}$ such that $\mathrm{Qcoh} \mathbb{D}(K)$ is equivalent to the category of modules over $\mathcal{A}\left(L^{*} \rightarrow\right.$ $\left.E^{\prime}\right)$.

Theorem 4.4.2. The kernel algebras $\mathcal{A}(G \rightarrow E)$ and $\mathcal{A}\left(L^{*} \rightarrow E^{\prime}\right)$ are FourierMukai dual to each other. Hence, we get an exact equivalence

$$
D(\mathrm{Q} \operatorname{coh} K) \simeq D(\mathrm{Q} \operatorname{coh} \mathbb{D}(K)),
$$

which also induces an equivalence between the bounded derived categories of coherent sheaves in the case $\Delta=\Delta_{S}^{\mathrm{fin}}$.

Proof. Set $H=L^{*}$. By construction, the extension $E \rightarrow A$ by $L$ is dual to the homomorphism $f^{\prime}: H \rightarrow \hat{A}$ (in the sense of Lemma 3.5.3), while the extension $E^{\prime} \rightarrow \hat{A}$ by $G^{*}$ is dual to the homomorphism $f: G \rightarrow A$. Applying Corollary 3.5.5 to the $G$-equivariant $L$-torsor $E \rightarrow A$ (resp. $H$-equivariant $G^{*}$-torsor $E^{\prime} \rightarrow \hat{A}$ ), we get isomorphisms of kernel algebras on $A$ and on $\hat{A}$ :

$$
\begin{aligned}
\mathcal{A}(G \rightarrow E) & \simeq \mathcal{A}_{A}^{G \times H}\left(\mathscr{L}\left(f^{\prime}\right)\right), \\
\mathcal{A}\left(H \rightarrow E^{\prime}\right) & \simeq \mathcal{A}_{\hat{A}}^{G \times H}(\mathscr{L}(f)),
\end{aligned}
$$

Here we use the notation of Corollary 3.7.4. It remains to apply this corollary. 
4.5. Fourier-Mukai duality for twisted sheaves. As in the previous section we work with generalized 1-motives of type $\Delta \in\left\{\Delta_{S}^{\text {fin }}, \Delta_{k}^{\text {for }}\right.$. Let $K$ be such a generalized 1-motive. Let also $G$ be an object of For, so $G$ is a finite flat commutative group scheme over $S$ if $\Delta=\Delta_{S}^{\text {fin }}$, and $G$ is a $k$-formal group if $\Delta=\Delta_{k}^{\text {for }}$. The definition of twisting data from Section 3.7 has an obvious extension to this situation.

Definition. A $G$-twisting data $T=\left(f, f^{\prime}, \alpha, \iota\right)$ for $K$ consists of homomorphisms $f: G \rightarrow K, f^{\prime}: G \rightarrow \mathbb{D}(K)$, and of a line bundle $\alpha$ over $G$ equipped with an isomorphism of 2-cocycles $\iota: \Lambda(\alpha) \simeq\left(f \times f^{\prime}\right)^{*} \mathcal{P}$, where $\mathcal{P}$ is the Poincaré biextension. If $T=\left(f, f^{\prime}, \alpha, \iota\right)$ is a $G$-twisting data for $K$ then the dual $G$-twisting data for $\mathbb{D}(K)$ is $\mathbb{D}(T)=\left(f^{\prime},-f, \hat{\alpha}, \iota^{\prime}\right)$, where $\hat{\alpha}$ is given by (3.7.7) and $\iota^{\prime}$ is induced by $\iota$.

We will use $G$-twisting data as above to define twisted versions of the category of $G$-equivariant sheaves on $K$. By Proposition 4.3.2 (i), twisting data $T=\left(f, f^{\prime}, \alpha, \iota\right)$ can be equivalently described by the data $(f, \mathscr{B}, \alpha, \iota)$, where $f: G \rightarrow K$ is a homomorphism, $\mathscr{B}$ is a biextension of $G$ and $K$ by $\mathbb{G}_{m}, \alpha$ is a line bundle over $G$ and $\iota: \Lambda(\alpha) \simeq(f \times \mathrm{id})^{*} \mathbb{B}$ is an isomorphism of 2-cocycles.

Given a representation $K=[H \rightarrow E]$ let us consider the composition of $f: G \rightarrow$ $K$ with the corresponding morphism $K \rightarrow H$ [1]. This will give an extension

$$
0 \rightarrow H \rightarrow \tilde{H}(f) \rightarrow G \rightarrow 0
$$

such that the composition $\widetilde{H}(f) \rightarrow G \rightarrow K \rightarrow H[1]$ is zero. We claim that to a morphism $f$ one can canonically (up to an automorphism of $\widetilde{H}(f)$, compatible with the extension structure) associate a morphism of exact triangles

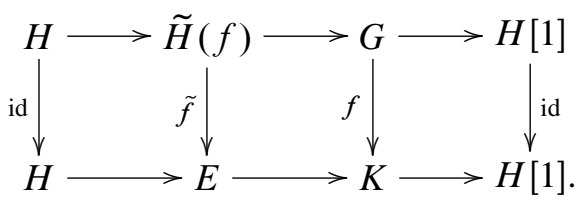

Indeed, a morphism in the derived category $f: G \rightarrow K$ can be represented by a map $\left[G_{1} \rightarrow G_{0}\right] \rightarrow[H \rightarrow E]$ in the homotopy category of complexes, where $G_{1} \subset G_{0}$ and $G_{0} / G_{1} \simeq G$. To such a map of complexes we associate the natural homomorphism $\left(G_{0} \oplus H\right) / G_{1} \rightarrow E$, identical on $H$. It remains to observe that one has a natural isomorphism $\left(G_{0} \oplus H\right) / G_{1} \simeq \widetilde{H}(f)$ of extensions of $G$ by $H$, and that our construction is compatible with the homotopy and with changing the complex $\left[G_{1} \rightarrow G_{0}\right]$. Mimicking the definition for the abelian varieties, let us consider the line bundle

$$
\mathscr{L}(T)=p_{1}^{*} \alpha \otimes \mathscr{B}
$$

on $G \times E$ (where $\mathcal{B}$ is the biextension coming from our twisting data). Its pull-back $\widetilde{\mathscr{L}}(T)$ to $\widetilde{H}(f) \times E$ has the natural structure of a 1-cocycle of $\widetilde{H}(f)$ with values in 
$\operatorname{Pic}(E)$, where the action of $\tilde{H}(f)$ on $E$ is induced by the homomorphism $\tilde{f}$. Now we define the twisted category of sheaves on $[K / G]$ associated with $T$ to be

$$
\operatorname{Qcoh}_{G}^{T}(K)=\mathcal{A}_{E}^{\tilde{H}(f)}(\tilde{\mathscr{L}}(T)) \text {-mod. }
$$

If we have another representation $K=\left[H^{\prime} \rightarrow E^{\prime}\right]$ such that $f: G \rightarrow K$ factors through $E^{\prime} \rightarrow K$, then we can construct another presentation $K=\left[H_{0} \rightarrow E_{0}\right]$ using Lemma 4.4.1 so that we have an exact triangle

$$
E_{0} \rightarrow E \oplus E^{\prime} \rightarrow K \rightarrow E_{0}[1] .
$$

This easily implies that $f: G \rightarrow K$ factors also through $E_{0} \rightarrow K$. Also, we have morphisms from $\left[H_{0} \rightarrow E_{0}\right]$ to the original two presentations of $K$. In this situation the corresponding 1-cocycle $\widetilde{\mathscr{L}}\left(T_{0}\right)$ of $\widetilde{H}_{0}(f)$ with values in $\operatorname{Pic}\left(E_{0}\right)$ is isomorphic to the pull-back of $\widetilde{\mathscr{L}}(T)$ with respect to the natural morphisms $\widetilde{H}_{0}(f) \rightarrow \widetilde{H}(f)$ and $E_{0} \rightarrow E$. Hence, we get an equivalence

$$
\mathcal{A}_{E_{0}}^{\tilde{H}_{0}(f)}\left(\tilde{\mathscr{L}}\left(T_{0}\right)\right)-\bmod \simeq \mathcal{A}_{E}^{\tilde{H}(f)}(\tilde{\mathscr{L}}(T))-\bmod
$$

(see Lemma 3.4.3), which shows that the right-hand side of (4.5.2) does not depend on the choice of a representation $K=[H \rightarrow E]$.

Now we will prove a generalization of Theorem 3.7.3 in this situation by constructing an equivalence of the derived categories of twisted sheaves on $[K / G]$ and $[\mathbb{D}(K) / G]$ associated with $T$ and $\mathbb{D}(T)$, respectively.

Theorem 4.5.1. In the above situation one has an exact equivalence

$$
D\left(\operatorname{Qcoh}_{G}^{T}(K)\right) \simeq D\left(\mathrm{Qcoh}_{G}^{\mathbb{D}(T)}(\mathbb{D}(K)) .\right.
$$

In the situation of orbi-abelian schemes (i.e., $\Delta=\Delta_{S}^{\mathrm{fin}}$ ), this equivalence induces an equivalence between the bounded derived categories of coherent sheaves.

Proof. We start by choosing dual representations for $K$ and $\mathbb{D}(K)$. Namely, let $K=\left[H_{1} \rightarrow E\right]$ be a representation of $K$, where $H_{1} \in$ For and $E$ is an extension

$$
0 \rightarrow L_{1} \rightarrow E \rightarrow A \rightarrow 0
$$

where $A \in \mathcal{A} b$ and $L_{1} \in \mathcal{A} f f$. Consider the corresponding representation for the dual 1-motive $\mathbb{D}(K)=\left[H_{2} \rightarrow E^{\prime}\right]$, where $E^{\prime}=\mathbb{D}\left[H_{1} \rightarrow A\right]$ and $H_{2}=L_{1}^{*}$, so that we have an exact sequence

$$
0 \rightarrow L_{2} \rightarrow E^{\prime} \rightarrow \hat{A} \rightarrow 0
$$

with $L_{2}=H_{1}^{*}$. 
We want to express everything in terms of kernel algebras over $A$ and $\hat{A}$ so that the dual sides enter into the picture in a symmetric way (as in Theorem 3.7.3). Recall that the two categories we want to compare are

$$
\begin{aligned}
\operatorname{Qcoh}_{G}^{T}(K) & =\mathcal{A}_{E}^{\tilde{H}_{1}(f)}(\tilde{\mathscr{L}}(T)) \text {-mod, } \\
\operatorname{Qcoh}_{G}^{\mathbb{D}(T)}(\mathbb{D}(K)) & =\mathcal{A}_{E^{\prime}}^{\widetilde{H}_{2}\left(f^{\prime}\right)}(\tilde{\mathscr{L}}(\mathbb{D}(T)) \text {-mod }),
\end{aligned}
$$

where $\widetilde{H}_{1}(f)$ and $\widetilde{H}_{2}\left(f^{\prime}\right)$ are extensions of $G$ corresponding to the composed morphisms $G \stackrel{f}{\rightarrow} K \rightarrow H_{1}[1]$ and $G \stackrel{f^{\prime}}{\rightarrow} \mathbb{D}(K) \rightarrow H_{2}[1]$, respectively. Recall that to define the relevant 1-cocycles we also use the morphisms $\tilde{f}: \widetilde{H}_{1}(f) \rightarrow E$ and $\tilde{f}^{\prime}: \widetilde{H}_{2}\left(f^{\prime}\right) \rightarrow E^{\prime}$ lifting $f: G \rightarrow K$ and $f^{\prime}: G \rightarrow \mathbb{D}(K)$, respectively.

Let $\widetilde{G}\left(f, f^{\prime}\right)$ denote the extension of $G$ by $H_{1} \oplus H_{2}$ such that the corresponding class in $\operatorname{Ext}^{1}\left(G, H_{1} \oplus H_{2}\right) \simeq \operatorname{Ext}^{1}\left(G, H_{1}\right) \oplus \operatorname{Ext}^{1}\left(G, H_{2}\right)$ has components represented by the extensions $\widetilde{H}_{1}(f)$ and $\widetilde{H}_{2}\left(f^{\prime}\right)$. Then we have an exact sequence

$$
0 \rightarrow H_{2} \rightarrow \widetilde{G}\left(f, f^{\prime}\right) \rightarrow \widetilde{H}_{1}(f) \rightarrow 0 .
$$

Recall that $H_{2}$ is Cartier dual to $L_{1}$, so this extension is dual (in the sense of Lemma 3.5.3 (i)) to some biextension $\mathscr{B}_{1}$ of $\widetilde{H}_{1}(f) \times L_{1}$ by $\mathbb{G}_{m}$. It is easy to check that $\mathscr{B}_{1}$ is isomorphic to the pull-back of the biextension $\mathcal{B}$ of $G \times E$ (corresponding to the homomorphism $f^{\prime}: G \rightarrow \mathbb{D}(K)$ ) under the natural homomorphism

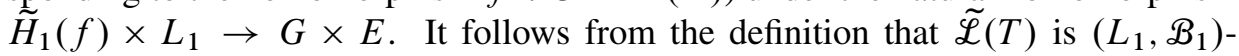
equivariant. Hence, we are in the situation of Proposition 3.5.4, where we view $E$ as an $L_{1}$-torsor over $A$. Using this Proposition we obtain an isomorphism

$$
(\phi \times \phi)_{*} \mathcal{A}_{E}^{\widetilde{H}_{1}(f)}(\tilde{\mathscr{L}}(T)) \simeq \mathcal{A}_{A}^{\widetilde{G}\left(f, f^{\prime}\right)}\left(\tilde{\mathscr{L}}_{1}\right),
$$

of kernel algebras over $A$, where $\phi: E \rightarrow A$ is the projection and $\tilde{\mathscr{L}}_{1}$ is the 1cocycle of $\widetilde{G}\left(f, f^{\prime}\right)$ with values in $\operatorname{Pic}(A)$ associated with $\widetilde{\mathscr{L}}(T)$ by the construction of Proposition 3.5.4. Thus, by Lemma 2.2.6, we get an equivalence

$$
\operatorname{Qcoh}_{G}^{T}(K) \simeq \mathcal{A}_{A}^{\widetilde{G}\left(f, f^{\prime}\right)}\left(\tilde{\mathscr{L}}_{1}\right) \text {-mod. }
$$

It is not hard to check that in fact $\widetilde{\mathscr{L}}_{1}$ comes from a natural $\widetilde{G}\left(f, f^{\prime}\right)$-twisting data $\widetilde{T}_{1}$ for $A$. More precisely, this twisting data consists of homomorphisms

$$
\boldsymbol{f}: \widetilde{G}\left(f, f^{\prime}\right) \rightarrow \widetilde{H}_{1}(f) \stackrel{\tilde{f}}{\rightarrow} E \rightarrow A, \quad \boldsymbol{f}^{\prime}: \widetilde{G}\left(f, f^{\prime}\right) \rightarrow \widetilde{H}_{2}\left(f^{\prime}\right) \stackrel{\tilde{f}^{\prime}}{\rightarrow} E^{\prime} \rightarrow \hat{A},
$$

and of the line bundle $\pi^{*} \alpha$ on $\widetilde{G}\left(f, f^{\prime}\right)$, where $\pi: \widetilde{G}\left(f, f^{\prime}\right) \rightarrow G$ is the projection, equipped with the isomorphism $\Lambda\left(\pi^{*} \alpha\right) \simeq\left(f \times f^{\prime}\right)^{*} \mathcal{P}$ induced by $\iota$. To see that the corresponding 1-cocycle of $\widetilde{G}\left(f, f^{\prime}\right)$ is isomorphic to $\widetilde{\mathscr{L}}_{1}$ one should look at their pull-backs to $\widetilde{G}\left(f, f^{\prime}\right) \times E$ and observe that these pull-backs are both isomorphic to 
the pull-back of the line bundle $\mathscr{L}(T)$ on $G \times E$ given by (4.5.1). Indeed, this follows from the fact that the pull-back of $\mathcal{B}$ to $\widetilde{G}\left(f, f^{\prime}\right) \times E$ coincides with the pull-back under the homomorphism $\widetilde{G}\left(f, f^{\prime}\right) \times E \rightarrow E^{\prime} \times A$ of the natural biextension of $E^{\prime} \times A$. It is easy to check that the above isomorphism on $\widetilde{G}\left(f, f^{\prime}\right) \times E$ is compatible with the $L_{1}$-action and with the 1-cocycle structures.

Next, we should repeat the above procedure for the dual data $(\mathbb{D}(K), \mathbb{D}(T))$. Note that the extension $\widetilde{G}\left(f, f^{\prime}\right)$ will get replaced by $\widetilde{G}\left(f^{\prime},-f\right)$ (an extension of $G$ by $H_{2} \oplus H_{1}$ ) which maps to $\widetilde{H}_{2}\left(f^{\prime}\right)$ (an extension of $G$ by $H_{2}$ ) and to $\widetilde{H}_{1}(-f)$ (an extension of $G$ by $H_{1}$ ). The result will be an isomorphism similar to (4.5.3),

$$
\left(\phi^{\prime} \times \phi^{\prime}\right)_{*} \mathcal{A}_{E}^{\widetilde{H}_{2}\left(f^{\prime}\right)}(\tilde{\mathscr{L}}(T)) \simeq \mathcal{A}_{\hat{A}}^{\widetilde{G}\left(f^{\prime},-f\right)}\left(\tilde{\mathscr{L}}_{2}\right),
$$

of kernel algebras over $\hat{A}$, where $\phi^{\prime}: E^{\prime} \rightarrow \hat{A}$ is the projection, and $\tilde{\mathscr{L}}_{2}$ is the 1-cocycle of $\widetilde{G}\left(f^{\prime},-f\right)$ with values in $\mathcal{P} i c(\hat{A})$ coming from a $\widetilde{G}\left(f^{\prime},-f\right)$-twisting data $\widetilde{T}_{2}$ for $\hat{A}$ that is defined similarly to $\widetilde{T}_{1}$. Again, by Lemma 2.2 .6 , this gives an equivalence

$$
\operatorname{Qcoh}_{G}^{\mathbb{D}(T)}(\mathbb{D}(K)) \simeq \mathcal{A}_{\hat{A}}^{\widetilde{G}\left(f^{\prime},-f\right)}\left(\tilde{\mathscr{L}}_{2}\right) \text {-mod. }
$$

We have a natural isomorphism $\sigma: \widetilde{H}_{1}(f) \rightarrow \widetilde{H}_{1}(-f)$ inducing [-1] on $H_{1}$ and identity on $G$. Since $\widetilde{G}\left(f^{\prime},-f\right)$ is built from the extensions $\widetilde{H}_{2}\left(f^{\prime}\right)$ and $\widetilde{H}_{1}(-f)$, we get the induced isomorphism

$$
\tau: \widetilde{G}\left(f, f^{\prime}\right) \rightarrow \widetilde{G}\left(f^{\prime},-f\right)
$$

compatible with the projections to $\widetilde{H}_{2}\left(f^{\prime}\right)$ and fitting into the commutative square

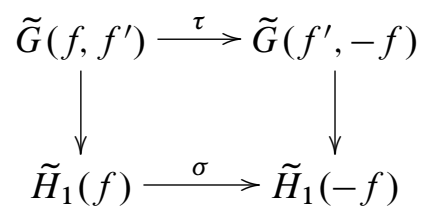

The map $\widetilde{G}\left(f^{\prime},-f\right) \rightarrow A$ forming a part of the twisting data $\widetilde{T}_{2}$ is given by the composition

$$
\widetilde{G}\left(f^{\prime},-f\right) \rightarrow \widetilde{H}_{1}(-f) \stackrel{h}{\rightarrow} E \rightarrow A,
$$

where $h$ fits into the morphism of exact triangles

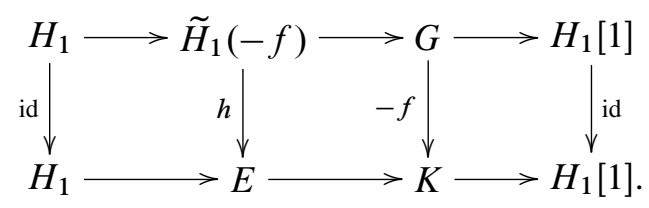

It follows that the composition $h \circ \sigma: \widetilde{H}_{1}(f) \rightarrow E$ differs from $-\tilde{f}$ by an automorphism of $\widetilde{H}_{1}(f)$, inducing identity on $H_{1}$ and $G$. Adjusting $\sigma$ (and hence $\tau$ ) 
by this automorphism we can assume that $h \circ \sigma=-\tilde{f}$. This implies that under the isomorphism $\tau: \widetilde{G}\left(f, f^{\prime}\right) \rightarrow \widetilde{G}\left(f^{\prime},-f\right)$ the map (4.5.4) gets identified with $-\boldsymbol{f}$. On the other hand, since $\tau$ is compatible with the projections to $\widetilde{H}_{2}\left(f^{\prime}\right)$, the map $\widetilde{G}\left(f^{\prime},-f\right) \rightarrow \hat{A}$ forming a part of the twisting data $\widetilde{T}_{2}$ gets identified under $\tau$ with $f^{\prime}$. It is easy to see from this that under the isomorphism $\tau$ the data $\widetilde{T}_{2}$ gets identified with the dual twisting data to $\widetilde{T}_{1}$. Now the required equivalence follows from Theorem 3.7.3.

Remark. One can generalize the notion of a $G$-twisting data to allow $G$ to be noncommutative (as we did in Section 3.7). Namely, let $G$ be a finite flat group scheme over $S$ (resp. a formal group scheme, ldu-pf over $k$ such that the action of $\mathrm{Gal}(\bar{k} / k)$ on $G(\bar{k})$ factors through $\operatorname{Gal}\left(k^{\prime} / k\right)$ for some finite field extension $\left.k \subset k^{\prime}\right)$ equipped with a homomorphism $\pi: G \rightarrow G_{0}$ with $G_{0} \in \mathcal{F}$ or. Then we can consider twisting data consisting of morphisms $f: G_{0} \rightarrow K, f^{\prime}: G_{0} \rightarrow \mathbb{D}(K)$, and of a line bundle $\alpha$ over $G$ equipped with an isomorphism of 2-cocycles $\iota: \Lambda(\alpha) \simeq\left(f \pi \times f^{\prime} \pi\right)^{*} \mathcal{P}$ over $G \times G$. One can still define the dual twists of the categories of $G$-equivariant sheaves on $K$ and on $\mathbb{D}(K)$ and prove the corresponding equivalence of derived categories.

Example. Let $k$ be a field of characteristic zero. Let us denote by $\mathbb{G}_{a}$ the additive group over $k$ and by $F$ the formal group obtained as the formal completion of $\mathbb{G}_{a}$ at zero. Let us say that an extension of an abelian variety $A$ over $k$ by $\mathbb{G}_{a}^{n}$ is universal if the corresponding homomorphism $F^{n} \rightarrow \hat{A}$ induces an isomorphism of formal completions at zero (all such extensions are canonically isomorphic). Now let us consider a generalized 1-motive of the form $K=\left[F^{n} \stackrel{\delta}{\rightarrow} E\right]$, where $\pi: E \rightarrow A$ is a universal extension of an abelian variety $A$ of dimension $n$ by $\mathbb{G}_{a}^{n}$, and the morphism $\delta \circ \pi: F^{n} \rightarrow A$ induces an isomorphism of formal completions at zero. It is easy to see that the dual generalized 1-motive $\mathbb{D}(K)$ is of the same type. Let $\mathbb{D}(K)=\left[F^{n} \stackrel{\delta^{\prime}}{\rightarrow} E^{\prime}\right]$, where $\pi^{\prime}: E^{\prime} \rightarrow \hat{A}$ is an extension of $\hat{A}$ by $\mathbb{G}_{a}^{n}$. Now let $G$ be a formal group scheme over $k$, isomorphic as a formal scheme to $\left.\operatorname{Spf}\left(k\left[t_{1}, \ldots, t_{n}\right]\right]\right)$, and consider $G$-twisting data $T$ for $K$ with $f$ and $f^{\prime}$ factoring as

$$
G \stackrel{\phi}{\rightarrow} E \rightarrow K, \quad G \stackrel{\phi^{\prime}}{\rightarrow} E^{\prime} \rightarrow \mathbb{D}(K) .
$$

Recall that, by Proposition 4.3.2(iii)), the Poincaré biextension of $K \times \mathbb{D}(K)$ is represented by the biextension $\left(\pi \times \pi^{\prime}\right)^{*} \mathcal{P}$, where $\mathcal{P}$ is the Poincaré biextension of $A \times \hat{A}$. Therefore, the above $G$-twisting data for $K$ induces $G$-twisting data $\bar{T}=\left(\bar{f}, \bar{f}^{\prime}, \alpha, \iota\right)$ for $A$ with $\bar{f}=\pi \circ \phi: G \rightarrow A$ and $\bar{f}^{\prime}=\pi^{\prime} \circ \phi^{\prime}$. Conversely, given $G$-twisting data $\bar{T}$ for $A$, we can consider arbitrary liftings $\phi$ and $\phi^{\prime}$ of $\bar{f}$ and $\overline{f^{\prime}}$, respectively (provided they exist), to obtain a $G$-twisting data for $K$ as above. Note that the category $\mathrm{Q} \operatorname{coh}_{G}^{T}(K)\left(\operatorname{resp} . \operatorname{Qcoh}_{G}^{\mathbb{D}(T)}(\mathbb{D}(K))\right)$ is equivalent to the category of modules over a $D$-algebra $\mathcal{A}$ on $E$ (resp. $D$-algebra $\mathcal{A}^{\prime}$ on $E^{\prime}$ ). Furthermore, if $(\delta, \phi): F^{n} \times G \rightarrow E$ (resp. $\left.\left(\delta^{\prime}, \phi^{\prime}\right): F^{n} \times G \rightarrow E^{\prime}\right)$ induces an 
isomorphism of formal completions at zero then $\mathcal{A}$ (resp. $\mathcal{A}^{\prime}$ ) is a tdo. If in addition $\phi^{\prime}$ factors through $\delta^{\prime}: F^{n} \rightarrow E^{\prime}$ then $\mathcal{A}$ is isomorphic to the algebra of differential operators on $E$. Thus, we get a lifting of the picture described in section 7 of [44] (see Example after Theorem 3.7.3 on p. 233) to $D$-algebras on $E$ and $E^{\prime}$. For example, if $\bar{f}=\overline{f^{\prime}}=0$ and $\phi$ (resp. $\phi^{\prime}$ ) is given by a homomorphism $G \rightarrow \operatorname{ker}(\pi)=\mathbb{G}_{a}^{n}$ (resp. $G \rightarrow \operatorname{ker}\left(\pi^{\prime}\right)=\mathbb{G}_{a}^{n}$ ) inducing an isomorphism of formal completions at zero then we get a derived equivalence for the corresponding pair of tdo's on $E$ and $E^{\prime}$.

\section{References}

[1] L. Alonso Tarrío, A. Jeremías López, and J. Lipman, Local homology and cohomology on schemes. Ann. Sci. École Norm. Sup. (4) 30 (1997), 1-39. Zbl 0894.14002 MR 1422312 158,160

[2] L. Alonso Tarrío, A. Jeremías López, and J. Lipman, Duality and flat base change on formal schemes. In Studies in duality on Noetherian formal schemes and non-Noetherian ordinary schemes. Contemp. Math. 244, Amer. Math. Soc., Providence, RI, 1999, 3-90; Corrections to the paper: "Duality and flat base change on formal schemes". Proc. Amer. Math. Soc. 131 (2003), 351-357. Zbl 0953.14011 MR 1716706 MR 1933323 159, 160, $161,162,163,164,165,167$

[3] L. Alonso Tarrío, A. Jeremías López, M. Pérez Rodríguez, and M. J. Vale Gonsalves, The derived category of quasi-coherent sheaves and axiomatic stable homotopy. Adv. Math. 218 (2008), 1224-1252. Zbl 1149.14016 MR 2419383

[4] D. Arinkin and R. Bezrukavnikov, Perverse coherent sheaves. Moscow Math. J. 10 (2010), no. 1, 3-29. Zbl 05816979 MR 2668828180

[5] C. Bartocci, U. Bruzzo, and D. Hernández Ruipérez, Fourier-Mukai and Nahm transforms in geometry and mathematical physics. Progr. Math. 276, Birkhäuser, Boston 2009. Zbl 1186.14001 MR 2511017158

[6] K. Behrend and P. Xu, Differentiable stacks and gerbes. Preprint 2006. arXiv:math/0605694 212

[7] A. Beilinson and J. Bernstein, A proof of Jantzen conjectures. In I. M. Gelfand seminar, Adv. Soviet Math. 16, Part 1, Amer. Math. Soc., Providence, RI, 1993, 1-50. Zbl 0790.22007 MR 1237825 155, 180, 213, 233

[8] A. A. Beilinson, J. Bernstein, and P. Deligne, Faisceaux pervers. Astérisque 100 (1982). Zbl 0536.14011 MR 0751966236

[9] A. Beilinson and V. Drinfeld, Chiral algebras. Amer. Math. Soc. Colloq. Publ. 51, Amer. Math. Soc., Providence, RI, 2004. Zbl 1138.17300 MR 2058353 210, 213

[10] O. Ben-Bassat, Twisting derived equivalences. Trans. Amer. Math. Soc. 361 (2009), 54695504. Zbl 1177.14037 MR 2515820 198, 211, 212

[11] O. Ben-Bassat, Gerbes and the holomorphic Brauer group of complex tori. Preprint 2008. arXiv:0811.2746 213

[12] D. Ben-Zvi, J. Francis, and D. Nadler, Integral transforms and Drinfeld centers in derived algebraic geometry. J. Amer. Math. Soc. 23 (2010), 909-966. Zbl 1202.14015 MR 2669705154 
[13] V. G. Berkovič, The Brauer group of abelian varieties. Funkcional Anal. i Priložen. 6 (1972), no. 3, 10-15; English transl. Functional Anal. Appl. 6 (1972), 180-184 Zbl 0264.14011 MR 0308134233

[14] A. Bondal and M. van den Bergh, Generators and representability of functors in commutative and noncommutative geometry. Mosc. Math. J. 3 (2003), 1-36. Zbl 1135.18302 MR 1996800236

[15] T. Bridgeland, Equivalences of triangulated categories and Fourier-Mukai transforms. Bull. London Math. Soc. 31 (1999), 25-34. Zbl 0937.18012 MR 1651025

[16] J.-L. Brylinski, Gerbes on complex reductive Lie groups. Preprint 2000. arXiv:math/0002158 198, 211, 212

[17] A. Canonaco and P. Stellari, Twisted Fourier-Mukai functors. Adv. Math. 212 (2007), 484-503. Zbl 1116.14009 MR 2329310154

[18] P. Deligne, La formule de dualité globale. In Théorie des topos et cohomologie étale des schémas, Séminaire de Géométrie Algébrique du Bois Marie 1963/64, Théorie des topos et cohomologie étale des schémas (SGA 4), TOME 3, Exp. XVIII, Lecture Notes in Math. 305. Springer-Verlag, Berlin 1973, 481-587. Zbl 0259.14006 172, 173, 239

[19] P. Deligne, Théorie de Hodge, III. Inst. Hautes Études Sci. Publ. Math. 44 (1974), 5-77. Zbl 0237.14003 MR 0498552240

[20] P. Deligne, Le symbole modéré. Inst. Hautes Études Sci. Publ. Math. 73 (1991), 147-181. Zbl 0749.14011 MR 1114212218

[21] M. Demazure, Lectures on p-divisible groups. Lecture Notes in Math. 302, SpringerVerlag, Berlin 1972. Zbl 0247.14010 MR 0344261199

[22] P. Gabriel, Généralités sur les groupes algébriques. In Propriétés générales des schémas en groupes. Séminaire de Géométrie Algébrique du Bois Marie 1962/64, Schémas en groupes (SGA 3), TOME I, Exp. $\mathrm{VI}_{\mathrm{A}}$, Lecture Notes in Math. 151, Springer-Verlag, Berlin 1970, 287-317. MR 0225784235

[23] P. Gabriel, Étude infinitésimale de des schémas en groupes: Groupes formels. In Propriétés générales des schémas en groupes. Séminaire de Géométrie Algébrique du Bois Marie 1962/64, Schémas en groupes (SGA 3), TOME I, Exp. VII ${ }_{\mathrm{B}}$, Lecture Notes in Math. 151, Springer-Verlag, Berlin 1970, 476-562. MR 0213358199

[24] R. Godement, Topologie algébrique et théorie des faisceaux. Hermann, Paris 1958. Zbl 0080.16201 MR 0102797157

[25] A. Grothendieck and J. Dieudonné, Eléments de géométrie algébrique I. Grundlehren Math. Wiss. 166, Springer-Verlag, Berlin 1971. Zbl 0203.23301 157, 159, 160

[26] A. Grothendieck, Technique de descente et théorème d'existence en géométri algébrique. V. Les schémas de Picard: théorèmes d'existence. Sém. Bourbaki 7 (1961/62), Exp. No. 232, 143-161. Zbl 0238.14014 MR 1611170 http://www.numdam.org/item?id=SB_1961-1962_7_143_0 213

[27] A. Grothendieck, Le groupe de Brauer. III. Exemples et compléments. In Dix exposés sur la cohomologie des schémas, North-Holland, Amsterdam 1968, 88-188. Zbl 0198.25901 MR 244271213 
[28] A. Grothendieck, Biextensions de faisceaux de groupes. In Groupes de monodromie en géométrie algébrique, Séminaire de Géométrie Algébrique du Bois Marie 1967-1969, Groupes de monodromie en géométrie algébrique (SGA 7 I), Exp. VII, Lecture Notes in Math. 288. Springer-Verlag, Berlin 1972, 133-217. Zbl 0247.14011 173

[29] A. Grothendieck, Complements sur les biextensions. Proprietes generales des biextensions des schemas en groupes. In Groupes de monodromie en géométrie algébrique, Séminaire de Géométrie Algébrique du Bois Marie 1967-1969, Groupes de monodromie en géométrie algébrique (SGA 7 I), Exp. VIII, Lecture Notes in Math. 288. SpringerVerlag, Berlin 1972, 218-312. Zbl 0221.14026 173, 174

[30] D. Happel, I. Reiten, and S. O., Tilting in abelian categories and quasitilted algebras. Mem. Amer. Math. Soc. 120 (1996), no. 575. Zbl 0849.16011 MR 1327209236

[31] N. Hitchin, Lectures on special Lagrangian submanifolds. In Winter School on mirror symmetry, vector bundles and Lagrangian submanifolds (Cambridge, MA, 1999), AMS/IP Stud. Adv. Math. 23, Amer. Math. Soc., Providence, RI, 2001, 151-182. Zbl 1079.14522 MR 1876068211

[32] Y. Kawamata, Equivalences of derived categories of sheaves on smooth stacks. Amer. J. Math. 126 (2004), 1057-1083. Zbl 1076.14023 MR 2089082154

[33] G. Laumon, Transformation de Fourier generalisée. Preprint 1996. arXiv:alg-geom/9603004 153, 154, 155, 199, 235, 239

[34] M. Lieblich, Moduli of twisted sheaves. Duke Math. J. 138 (2007), 23-118. Zbl 1122.14012 MR 2309155211

[35] J. Lipman, Notes on derived functors and Grothendieck duality. In Foundations of Grothendieck duality for diagrams of schemes, Lecture Notes in Math. 1960, Springer, Berlin 2009, 1-259. Zbl 1163.14001 MR 2490557 157, 158, 161, 163, 164, 189

[36] J. S. Milne, Arithmetic duality theorems. 2nd ed., BookSurge, LLC, Charleston, SC, 2006. Zbl 1127.14001 MR 2261462 235

[37] D. Murfet, Derived categories of quasi-coherent sheaves. Preprint 2006. http://therisingsea.org/notes/DerivedCategoriesOfQuasicoherentSheaves.pdf 158

[38] S. Mukai, Duality between $D(X)$ and $D(\hat{X})$ with its application to Picard sheaves. Nagoya Math. J. 81 (1981), 153-175. Zbl 0417.14036 MR 607081 154, 175

[39] F. Oort, Commutative group schemes. Lecture Notes in Math. 15, Springer-Verlag, Berlin 1966. Zbl 0216.05603 MR 0213365 235, 236, 238

[40] D. O. Orlov, Equivalences of derived categories and K3 surfaces. J. Math. Sci. 84 (1997), 1361-1381. Zbl 0938.14019 MR 1465519154

[41] D. O. Orlov, Derived categories of coherent sheaves on abelian varieties and equivalences between them. Izv. Ross. Akad. Nauk Ser. Mat. 66 (2002), no. 3, 131-158; English transl. Izv. Math. 66 (2002), 569-594. Zbl 1031.18007 MR 1921811 233

[42] A. Polishchuk, Analogue of Weil representation for abelian schemes. J. Reine Angew. Math. 543 (2002), 1-37. Zbl 1065.11040 MR 1887877229

[43] A. Polishchuk, Abelian varieties, theta functions and the Fourier transform. Cambridge Tracts in Math.153, Cambridge University Press, Cambridge 2003. Zbl 1018.14016 MR 1987784232 
[44] A. Polishchuk and M. Rothstein, Fourier transform for D-algebras, I. Duke Math. J. 109 (2001), 123-146. Zbl 1069.14003 MR 1844207 153, 154, 155, 156, 175, 233, 234, 248

[45] J. Rickard, Morita theory for derived categories. J. London Math. Soc. (2) 39 (1989), 436-456. Zbl 0642.16034 MR 1002456154

[46] J.-P. Serre, Groupes proalgébriques. Inst. Hautes Études Sci. Publ. Math. 7 (1960), 341403. Zbl 0097.35901 MR 0118722238

[47] C. Simpson, The Hodge filtration on nonabelian cohomology. In Algebraic geometrySanta Cruz 1995, Proc. Sympos. Pure Math. 62, Part 2, Amer. Math. Soc., Providence, RI 1997, 217-281. Zbl 0914.14003 MR 1492538202

[48] N. Spaltenstein, Resolutions of unbounded complexes. Compositio Math. 65 (1988), 121-154. Zbl 0636.18006 MR 932640157

[49] B. Toën, The homotopy theory of $d g$-categories and derived Morita theory. Invent. Math. 167 (2007), 615-667. Zbl 1118.18010 MR 2276263154

[50] M. Van den Bergh, Non-commutative $\mathbb{P}^{1}$-bundles over commutative schemes. Trans. Amer. Math. Soc., to appear. arXiv:math/0102005v3 155

[51] J.-L. Verdier, Des catégories dérivées des catégories abéliennes. Astérisque 239 (1996). Zbl 0882.18010 MR 1453167201

[52] A. Yekutieli, Smooth formal embeddings and the residue complex. Canad. J. Math. 50 (1998), 863-896. Zbl 0932.14007 MR 1638635162

Received January 1, 2009; revised September 18, 2009

A. Polishchuk, Department of Mathematics, University of Oregon, Eugene, OR 97405, U.S.A.

E-mail: apolish@math.uoregon.edu 